\title{
Approximation of Baker domains and convergence of Julia sets
}

\author{
Dissertation \\ zur Erlangung des Doktorgrades \\ der Fakultät für Mathematik und Informatik \\ der Georg-August-Universität zu Göttingen
}

\author{
vorgelegt von \\ Tania Garfias-Macedo \\ aus \\ Mexiko Stadt, Mexiko
}

Göttingen 2012 
D7

Referent: PD. Dr. H. Kriete

Korreferent: Prof. Dr. V. Pidstrygach

Tag der mündlichen Prüfung: 25.10.2012 
Para Lucia, por su fortaleza. 1915-2011 



\section{Contents}

\begin{tabular}{ll}
\hline Motivation and main results & 1
\end{tabular}

1 Preliminaries and known results 13

1.1 Preliminaries from complex analysis . . . . . . . . . . . . . . 14

1.2 Singular values $\ldots \ldots \ldots \ldots \ldots$

$1.3 \quad$ Fixed and periodic points . . . . . . . . . . . . . . . . 17

1.4 Fatou and Julia sets . . . . . . . . . . . . . . . . . . . 17

1.5 Fatou components . . . . . . . . . . . . . . . . . . . . 19

1.6 Singular values and Fatou components . . . . . . . . . . . . . . . 21

1.7 On Baker domains and wandering domains . . . . . . . . . . . . . 22

1.8 On the polynomial-like mappings . . . . . . . . . . . . . 26

1.9 Hausdorff and kernel convergence . . . . . . . . . . . . . . 27

1.10 Notation . . . . . . . . . . . . . . . . . . . . . . 30

$2 \quad$ Dynamical description of the family $g_{\lambda, \mu} \quad 31$

2.1 Basin of attraction $\mathcal{A}_{\mu} \ldots \ldots \ldots \ldots \ldots$

2.2 Dynamics on the real line . . . . . . . . . . . . . . . . . . . 35

$2.2 .1 \quad$ Dynamics for $\lambda>0$. . . . . . . . . . . . 37

2.2 .2 Dynamics for $\lambda<0 \quad \ldots \ldots \ldots \ldots$. . . . . . . . . . . 41

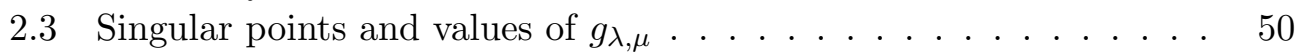

2.4 Absorbing domain $\mathcal{H}_{\eta, \rho} \quad \ldots \ldots \ldots \ldots$. . . . . . . . . . . . 60

$2.4 .1 \quad$ First approach by $\mathcal{M}_{\delta, \mu} \ldots \ldots \ldots$. . . . . . . . . 62

2.4 .2 Invariance of domain $\mathcal{H}_{\eta, \rho}$ under $g_{\lambda, \mu} \ldots \ldots \ldots . \ldots 64$

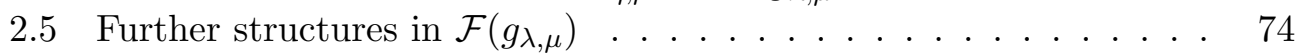

2.6 Polynomial-like mapping . . . . . . . . . . . . . . . . . . 77

\begin{tabular}{|lll}
3 & Convergence of the families $g_{\lambda, \mu}$ to $g_{\lambda}$ & $\mathbf{8 7}$
\end{tabular}

$3.1 \quad$ Some results about the family $g_{\lambda} \ldots \ldots \ldots \ldots$. . . . . . . . . . 88

3.2 Approximation of indifferent fixed points . . . . . . . . . . . . . . 91 
3.3 Kernel convergence on the Fatou set . . . . . . . . . . . . . . 100

3.3 .1 Kernel convergence to the Baker domain $\mathcal{B}_{\lambda} \ldots$. . . . . . . 101

3.3 .2 Kernel convergence to the parabolic basin $\mathcal{D}_{p} \ldots \ldots$. . . . 107

3.4 Filled Julia sets . . . . . . . . . . . . . . . . . . . . . . 120

3.5 Hausdorff convergence of Julia sets . . . . . . . . . . . . . 123

\begin{tabular}{lr}
\hline Outlook & 129
\end{tabular}

\begin{tabular}{lr}
\hline Bibliography & 133
\end{tabular} 


\section{Motivation and main results}

The area of holomorphic dynamics deals with the iteration of holomorphic functions, that is, complex functions of one complex variable which are differentiable wherever the function is defined. The study of iteration of functions was principally developed by Fatou and Julia in the early 20's. Even though their inspiration may lie behind in the 19th century in the work of Henri Poincaré who used iteration in his studies of celestial mechanics. Another starting point for the theory lies on the work of Ernst Schröder who showed the existence of attracting basins, now and then named after him.

In the beginning of the 1920's Pierre Fatou and Gaston Julia worked mainly with the iteration of rational functions and set down a large number of the fundamental results for such functions. Later in 1926 Fatou began the study of iteration of transcendental entire functions giving examples and establishing the significant differences to the theory developed for rational functions.

Fatou and Julia proved that the Julia set $\mathcal{J}$ of a rational function is the closure of the set of repelling periodic points, but they did it through different approaches. Fatou firstly showed that any point in $\mathcal{J}$ is the limit point of periodic points and afterwards showed that there exist only finitely many non-repelling points. On the contrary, Julia started by showing that the set of repelling or indifferent periodic points is not empty and from there he developed his theory on the closure of the set of repelling points. Both proofs have one or the other argument which cannot be generalised to transcendental functions. Fatou himself mentioned that it is of great difficulty to generalise the work of Julia to the transcendental case.

In the subsequent years there was a small development in the subject only. We mention, however, two historical works due to Cremer in 1925 and to Brolin in 1967. The two of them worked with iteration of rational functions only. Nevertheless, in the mid 1950's Noel Baker received the suggestion to work with functional equations and used much of the theory developed by Fatou and Julia, unknown by 
that time. He was strongly interested in the subject and continued with this research answering some of the questions concerning transcendental entire functions left open by Fatou.

Around 1980, the subject was reborn mainly because of the arrival of accessible computer graphics and the information alluded by them. By that time, Baker had completed the foundations begun by Fatou and Julia earlier, applying techniques of classical complex analysis.

One of the main differences in the iteration of rational functions and transcendental functions is the occurrence of two new types of dynamics or Fatou components, known as Baker domains and wandering domains. Baker showed the existence of wandering domains in 1976, which was one of Fatou's open questions. Some years later in 1983 Sullivan proved they do not exist for rational functions. Sullivan's proof of the No Wandering Domain Theorem used a new technique in the subject, the quasiconformal conjugacies. Baker succeeded to generalise this proof to certain families of transcendental functions.

Until now we mentioned the theory of dynamical systems in a classical sense, that is, looking at the iteration of a single function. There is, however, another basic problem in the theory that studies the changes of the iterative behaviour of a whole family of functions or for a given function under perturbation. In the present work we are concerned with the two latter approaches: on the one hand we study the dynamical behaviour of a family of transcendental entire functions, on the other hand, this family arises from the perturbation to a family of transcendental entire functions. We describe the minimal conditions such that we obtain stability of both the Julia and the Fatou sets under the perturbation.

The Fatou set $\mathcal{F}(f)$ of an entire function $f$ is the set of points in the complex plane where the sequence of iterates of $f$ forms a normal family in some neighbourhood of the point. The Julia set $\mathcal{J}(f)$ is the complement of $\mathcal{F}$ in the Riemann sphere. Roughly speaking $\mathcal{F}$ is the stable set and on $\mathcal{J}$ the dynamics are chaotic.

It is well-known that the convergence in a dynamical sense is not guaranteed only from the converge in an analytical sense, that is, when the functions converge uniformly on compact sets. There is a strong dependence on the properties of the limit function and it is well-known that attracting basins are stable under small perturbations. Hence the first idea is study stability after perturbing basins of attraction.

The first example where the dynamical convergence is satisfied is due to Devaney et 
al. [20]. Inspired by the uniform convergence of the polynomials $P(z)=\left(1+\frac{z}{d}\right)^{d}$ to the function $f(z)=e^{z}$, they showed that the exponential family $E(\lambda, z)=\lambda e^{z}$ is approximated by the family of polynomial $P_{d}(\lambda, z)=\lambda\left(1+\frac{z}{d}\right)^{d}$ in a dynamical sense as $d$ tends to infinity. In other words, the Julia set $\mathcal{J}(P)$ converges to $\mathcal{J}(E)$ in the Hausdorff metric. Fagella obtained the same result in [27] for the standard complex family $\mathcal{G}(\lambda, z)=\lambda z e^{z}$, which is approximated by the family of polynomial $Q_{d}(\lambda, z)=\lambda z\left(1+\frac{z}{d}\right)^{d}$.

It is worth to mention that in these cases the transcendental functions are of finite type, that is the set of singularities of the inverse function consists of finitely many points only. In particular, this implies that the limit functions have neither wandering domains nor Baker domains, and in this sense their dynamics resemble the dynamics of polynomials. However, the convergence is guaranteed for a wider class of functions.

A first general result due to Kisaka can be found in [36, where he assumes that the limit function $f$ is transcendental entire. This result was extended by Krauskopf and Kriete in [44] for meromorphic functions and says

Let $\left\{f_{n}\right\}_{n \in \mathbb{N}}$ converge to $f$ uniformly on compact subsets. If $\mathcal{F}(f)$ is the union of basins of attracting periodic orbits and $\infty \in \mathcal{J}(f)$, then $\left\{\mathcal{J}\left(f_{n}\right)\right\}_{n \in \mathbb{N}}$ converges to $\mathcal{J}(f)$ in the Hausdorff metric. The union may be empty, in which case $\mathcal{J}(f)=\widehat{\mathbb{C}}$.

The result is sharp, since there are counterexamples when the Fatou set of the limit function features other types of components. Notice that there is no restriction on the number of singular values. Lauber considered in [48] the functions $f(z)=$ $z-c+R(z) e^{a z}$ where $R(z)$ is a polynomial and $\operatorname{Re} a c>0$. He proved that if $f$ has no wandering domains, Siegel discs or parabolic basins and has only one Baker domain, then there exists a family of polynomials $P_{n}$ converging uniformly on compact subset to $f$ and for which the Julia sets converge in the Hausdorff metric. However, when $a c \in[1,2)$, then there exists a family of polynomials $Q_{n}$ for which the Julia set convergence is not satisfied. This example is a generalisation of a work due to Morosawa in [53. He presented the function $f(z)=z+e^{z}-1$ and approximations by two different families of polynomials showing positive and negative convergence on the Julia set.

At last we mention that Baker domains are not stable under perturbation. An example of this instability is due to Lauber in [49]. He studied the Fatou family $F(c, z)=z-c+e^{z}$, with $c=c_{1}+i c_{2}$, which has a Baker domain as a unique Fatou component when $c_{1}>0$. In contrast to this, if $c_{1}=0$, then $\mathcal{F}\left(f_{c}\right)$ consists of infinitely many different Baker domains, a Baker domain and its preimages, wandering domains, or it can even be empty. However, $\mathcal{J}\left(f_{c_{1}+i c_{2}}\right)$ converges to 
$\mathcal{J}\left(f_{i c_{2}}\right)$ in the Hausdorff metric as $c_{1}$ tends to zero. Hence the stability of Julia sets is stronger than the stability of the Fatou components.

One of the techniques used in the examples above is the logarithmic lift of functions, which is usually used to prove the existence of Baker domains. Starting with a self-map $g$ of $\mathbb{C}^{*}$ with known dynamics around the origin we can lift the dynamics via the exponential function. For a function $f$ and $\varphi(z)=e^{a z}$ we say that $f$ is the logarithmic lift of $g$ if $\varphi \circ f=g \circ \varphi$ holds for some appropriate $a \neq 0$. Such a function $f$ exists and is entire, since $g(\varphi(z)) \neq 0$ for every $z \in \mathbb{C}$. It was proved by Bergweiler in [13] that $\mathcal{J}(f)=\varphi^{-1}(\mathcal{J}(g))$.

In the view of the examples and results mentioned until now, we present our work. In a few words, we studied the approximation of a transcendental entire function featuring a Baker domain which cannot by constructed via logarithmic lift. Furthermore, the limit function has infinitely many singular values, that is, it is not of finite type, and may feature more than one Fatou component. We approximate these functions by transcendental entire functions, in contrast to most known examples, where the approximations are done through polynomials.

The starting point is the limit function

$$
g_{\lambda}(z)=z-1+\lambda z e^{z} \quad \text { with } \lambda \in \mathbb{C}^{*}
$$

featuring a unique Baker domain $\mathcal{B}_{\lambda}$. The function $g_{\lambda}$ has a free critical point, that is, the Baker domain $\mathcal{B}_{\lambda}$ contains all critical points except at most one, denoted by $c_{0}$. The dynamics of $c_{0}$ depend on the parameter $\lambda$ and in the case that $c_{0} \in \mathcal{B}_{\lambda}$, then it is the only Fatou component and it is completely invariant. Otherwise, $c_{0}$ may lie in a basin of attraction, a parabolic basin, a Siegel disc, a wandering domain or the Julia set. In the first three cases, we disclaim the existence of wandering domains. The existence of the Baker domain $\mathcal{B}_{\lambda}$ and further properties of the function $g_{\lambda}$ were proved by Lauber in his Dissertation [48. We present in Section 3.1 a discussion concerning the existence of wandering domains including a proof of the non-existence in the case that $g_{\lambda}$ has only the Baker domain or additionally a non-repelling cycle.

As mentioned above, we wonder how we can perturb the family of functions $g_{\lambda}$ in a way that we guarantee the Hausdorff convergence of the Julia sets. The answer is to approximate $\mathcal{B}_{\lambda}$ by basins of attraction, reached by multiplying the function by a contracting factor. Hence we define the functions

$$
g_{\lambda, \mu}(z)=(1-\mu)\left(z-1+\lambda z e^{z}\right) \quad \text { with } \mu \in\left(0, \frac{1}{2}\right) .
$$


We see that $g_{\lambda, \mu}$ converges uniformly on compact subsets of $\mathbb{C}$ to $g_{\lambda}$ as $\mu$ tends to zero.

There exists a $\mu_{1} \in\left(0, \frac{1}{2}\right)$ such that the function $g_{\lambda, \mu}$ has an attracting fixed point $z_{\mu}$ for every $\mu \leq \mu_{1}$ and lying in the interval $\left(-\frac{1}{\mu}, 2-\frac{1}{\mu}\right) \subset \mathbb{R}$. In order to give a first geometrical description of its basin of attraction $\mathcal{A}_{\mu}$ we construct an absorbing domain defined as the union of two smaller domains $\mathcal{H}_{\eta, \rho}:=\mathcal{H}_{\eta} \cup \mathcal{M}_{\rho}$. The subdomains are defined as $\mathcal{M}_{\rho}$ for points with $1-\frac{1}{2 \mu}<\operatorname{Re} z<x_{0}$ and $\mathcal{H}_{\eta}$ for points with $\operatorname{Re} z \leq 1-\frac{1}{2 \mu}$ in Equations 2.22 and 2.23 .

We prove in Section 2.4 the existence of a $\mu_{2} \in\left(0, \mu_{1}\right)$ such that the domain $\mathcal{H}_{\eta, \rho}$ is invariant under the function $g_{\lambda, \mu}$ for every $\mu \leq \mu_{2}$. One of the first steps to show the convergence of the Fatou sets is achieved by proving the convergence of the the absorbing domain $\mathcal{H}_{\eta, \rho}$ to an analogue absorbing domain $\mathcal{M}_{1 / 2}$ of the Baker domain $\mathcal{B}_{\lambda}$. Later in Subsection 3.3.1 this is a useful tool to prove the convergence of the attracting basin $\mathcal{A}_{\mu}$ to $\mathcal{B}_{\lambda}$ as follows.

Theorem 3.14. Kernel convergence to the Baker domain $\mathcal{B}_{\lambda}$. Let $\lambda \in \mathbb{C}^{*}$ be such that all critical values belong to the Baker domain $\mathcal{B}_{\lambda}$. Then $\mathcal{A}_{\mu}$ converge to $\mathcal{B}_{\lambda}$ as kernel in the sense of Carathéodory.

The set of singularities of a function, denoted by $\operatorname{sing}\left(f^{-1}\right)$, plays an important role in the theory of holomorphic dynamics, as we discuss in more detail in the following chapter in Section 1.6. For a transcendental entire function $f$ the set $\operatorname{sing}\left(f^{-1}\right)$ consists of critical values and asymptotic values. In our case, the function $g_{\lambda, \mu}$ has no finite asymptotic values, however $\infty$ might but not need to be an asymptotic value. The set of critical values of $g_{\lambda, \mu}$ equals the set of critical values of $g_{\lambda}$, since the zeros of the derivative remain invariant when we multiply the function by a constant. In particular, the set of critical points is countable, unbounded and has no accumulation points in $\mathbb{C}$. Furthermore, it is possible to introduce a labelling for the critical points with respect to their imaginary part, since they lie on a parametrised curve.

For parameters $\lambda \in \mathbb{C}^{*} \backslash \mathbb{R}^{+}$write $c_{k}$ for a critical point with $k \in \mathbb{Z}$. Then the following holds for every $\mu \in\left(0, \frac{1}{2}\right)$.

- For $k=0$ we have $\operatorname{Im} c_{0} \in(-\pi, \pi)$, and in particular $\operatorname{Im} c_{0}>0$ for $\operatorname{Im} \lambda>0$.

- For $k>0$ and $\operatorname{Im} \lambda \geq 0$ we have $\operatorname{Im} c_{k}>\pi$. For $k<0$ we have $\operatorname{Im} c_{k}<0$.

- In general $\operatorname{Im} c_{k}>\operatorname{Im} c_{j}$ if $k>j$.

- $\left|\operatorname{Im} c_{k}-\operatorname{Im} c_{k+1}\right|$ and $\left|c_{k}-c_{k+1}\right|$ tend to $2 \pi$ as $|k|$ tends to $\infty$. 
In particular, only a finite number of the critical points is contained in the absorbing domain $\mathcal{H}_{\eta, \rho}$. Hence we cannot guarantee that there are no other Fatou components besides $\mathcal{A}_{\mu}$ or the non-repelling cycle generated by the free critical point $c_{0}$.

For parameters $\lambda \in \mathbb{R}^{*}$ we separately prove that $g_{\lambda, \mu}$ has none, one or two different critical points lying on $\mathbb{R}$ if $\lambda>0$, while for $\lambda<0 g_{\lambda, \mu}$ has exactly one critical point on $\mathbb{R}$. In contrast to this, the number of fixed points lying on $\mathbb{R}$ presents the "opposite behaviour". For parameters $\lambda>0$ the function $g_{\lambda, \mu}$ has a unique fixed point, which is the known fixed point $z_{\mu}$. But for values of $\lambda<0$, the function $g_{\lambda, \mu}$ exhibits a bifurcation scheme.

There exist, besides the fixed point $z_{\mu}$, one or two fixed points which change their behaviour as the parameter $\lambda$ moves continuously on the real line forward $-\infty$. The fixed points go from no existing to the emergence of one parabolic fixed point, which then bifurcates into two fixed points, one attracting and one repelling. The repelling fixed point maintains its nature, while the multiplier of the attracting fixed point changes from having norm smaller than one, then equals one, and finally greater than one, maintaining its repelling nature afterwards for every parameter as $\lambda$ keeps decreasing. The bifurcation scheme continuous as the attracting condition is transferred from the latter parabolic fixed point to an attracting cycle of period two, and later to an attracting cycle of period four, and so on.

The behaviour of the free critical point $c_{0}$ changes in analogy to the behaviour of these periodic points. At the beginning, $c_{0}$ belongs to the basin of attraction $\mathcal{A}_{\mu}$, then to the parabolic and attracting basins that arise with the existence of the fixed points. Later, the critical point $c_{0}$ belongs to the new parabolic basin and then to the basin of attraction of period two, and so on. The critical point eventually lies in the Julia set $\mathcal{J}\left(g_{\lambda, \mu}\right)$ after the parameter $\lambda$ goes through all components of the copy of the Mandelbrot set.

The absorbing domain $\mathcal{H}_{\eta, \rho}$ described above is contained in a left half-plane. Moreover the attracting basin $\mathcal{A}_{\mu}$ features a larger structure which extends to the right hand side in form of horizontal strips. In order to describe it, we construct a family of invariant Jordan curves $\left\{\Gamma_{k, \mu}\right\}_{k}$ with $|k| \leq N, k \neq 0$ as follows.

Theorem 2.37 Structure Theorem. Let $c_{k}$ be a critical point and $v_{k}$ its value for some $k \in \mathbb{Z}^{*}$. Then there exists an $N \in \mathbb{N}, N=N(\mu)$, and a family of Jordan curves $\left\{\Gamma_{k, \mu}\right\}_{k}$ with $|k| \leq N, k \neq 0$, and $\Gamma_{k, \mu}: t \mapsto \gamma_{k}(t)$ for $t \in[0, \infty)$. The family $\left\{\Gamma_{k, \mu}\right\}_{k}$ has the following properties

i) For each $k$, there is exactly one critical point $c_{k}$ on $\Gamma_{k, \mu}$. 
ii) For each $\Gamma_{k, \mu}$ we have: $\gamma_{k}(0)=z_{\mu}$ and $\operatorname{Re}\left(\gamma_{k}(t)\right) \rightarrow \infty$ as $t \rightarrow \infty$ while $\operatorname{Im}\left(\gamma_{k}(t)\right)$ is bounded.

iii) The curves $\Gamma_{k, \mu} \backslash\left\{\gamma_{k}(0)\right\}$ are pairwise disjoint.

iv) For each $k$, the set $\Gamma_{k, \mu} \cup\left(-\infty, z_{\mu}\right)$ is completely invariant.

The existence of the invariant Jordan curves has several implications. It follows by continuity the existence of open neighbourhoods around each curve that belong to the Fatou set. Hence the Julia set $\mathcal{J}\left(g_{\lambda, \mu}\right)$ must be contained in strips that extend horizontal asymptotically to infinity for points with large real part illustrating a Cantor bouquet structure. Further Fatou components must be trapped as well in these strips. Even though we guarantee the existence and invariance only for a finite number of these curves depending on the parameter $\mu$, we prove the existence of countably many strips which belong to the basin of attraction $\mathcal{A}_{\mu}$ distributed horizontally above and below the curves $\Gamma_{k, \mu}$.

Of great importance is the construction of a polynomial-like triple $\left(g_{\lambda, \mu}, V, g_{\lambda, \mu}(V)\right)$ as follows, whose proof is mainly based on the properties of the Structure Theorem. The theorem implies, roughly speaking, that the dynamics of $g_{\lambda, \mu}$ locally resemble the iterates of a polynomial of the given degree.

Theorem 2.39 Polynomial-like Mapping. For every $d \in \mathbb{N}$ with $d \geq 2$ and any given $\lambda \in \mathbb{C}^{*} \backslash \mathbb{R}^{+}$there exists a sufficiently small $\mu_{0} \in\left(0, \frac{1}{2}\right)$ and a domain $V$ such that the critical points $c_{k} \in V$ for $-d<k<d$ and the triple $\left(g_{\lambda, \mu}, V, g_{\lambda, \mu}(V)\right)$ is a polynomial-like mapping of degree at most $2 d$ for every $\mu \leq \mu_{0}$.

After giving a detailed description of the dynamics of $g_{\lambda, \mu}$, which depends mainly on the parameter $\lambda$, we deal with the convergence of the Fatou and Julia sets as $\mu$ tends to zero. In order to give an appropriate concept of convergence in a dynamical sense, there exist in the literature two kinds of convergence. The first regards open sets and is known as kernel convergence in the sense of Carathéodory. The second is the Hausdorff convergence of compact sets. We refer the reader to Section 1.9 for detailed definitions and a discussion on the topic.

As described above the function $g_{\lambda, \mu}$ has a free critical point which may belong to the attracting basin $\mathcal{A}_{\mu}$ or to other Fatou components as we show in the detail in the case $\lambda \in \mathbb{R}^{*}$. The function $g_{\lambda}$ features an analogue behaviour of the free critical point. In the case that $g_{\lambda}$ has an indifferent fixed point $z_{p}$, we show the existence of a sequence of attracting fixed points $z_{p, \mu}$ of $g_{\lambda, \mu}$ which converges to $z_{p}$. Furthermore we prove the convergence of the attracting basins to parabolic basins in the case that these indifferent fixed points are parabolic. 
Theorem 3.18 Kernel convergence to parabolic basins of fixed points. Let $g_{\lambda}$ and $g_{\lambda, \mu}$ be as described above. Then for every parabolic fixed point $z_{p} \in$ $\partial D\left(-\frac{2}{3}, \frac{1}{3}\right)$ and every $\mu \leq \mu_{*}$, the attracting basins $\mathcal{A}_{p, \mu}$ converge as kernels to the parabolic basin $\mathcal{D}_{p}$ as $\mu$ tends to zero.

In the case that the Fatou set of the limit function $g_{\lambda}$ has no parabolic cycles and no wandering domains, we prove a slightly stronger result than the Hausdorff convergence of the Julia set. To this end we define the filled Julia set for the function $g_{\lambda}$ as

$$
\mathcal{K}\left(g_{\lambda}\right):=\mathbb{C} \backslash \mathcal{B}_{\lambda} .
$$

For the approximating functions $g_{\lambda, \mu}$ we define

$$
\mathcal{K}\left(g_{\lambda, \mu}\right):=\mathbb{C} \backslash \mathcal{A}_{\mu}
$$

where $\mathcal{A}_{\mu}$ is the attracting basin converging to $\mathcal{B}_{\lambda}$ as $\mu$ tends to zero. With this, we prove the following result.

Theorem 3.26 Convergence of filled Julia sets. Let $\lambda \in \mathbb{C}^{*}$ be arbitrary but fixed such that $g_{\lambda}$ has no wandering domains and no parabolic cycles. Then $\mathcal{K}\left(g_{\lambda, \mu}\right)$ converges to $\mathcal{K}\left(g_{\lambda}\right)$ in the Hausdorff metric as $\mu$ tends to zero.

Finally all results presented until now conduce to prove the convergence of the Julia sets in the Hausdorff metric.

Theorem 3.27 Hausdorff Convergence of Julia sets. Let $\lambda \in \mathbb{C}^{*}$. If the function $g_{\lambda}$ satisfies one of the following conditions

- $\mathcal{F}\left(g_{\lambda}\right)$ consists only of the Baker domain $\mathcal{B}_{\lambda}$,

- $g_{\lambda}$ has an attracting periodic cycle (of any period),

- $g_{\lambda}$ has a Cremer cycle (of any period),

- $g_{\lambda}$ has a Siegel disc, which centre is an indifferent fixed point, or

- $g_{\lambda}$ has a parabolic fixed point,

then $\mathcal{J}\left(g_{\lambda, \mu}\right)$ converges to $\mathcal{J}\left(g_{\lambda}\right)$ in the Hausdorff metric as $\mu$ tends to zero.

For fixed $\lambda \in \mathbb{C}^{*}$ at most one of these conditions can be satisfied.

In Chapter 1 we give a small introduction to the theory of holomorphic dynamics and some known results which we use during the present work. We include as well a detailed definition of convergences on the one hand of open sets as kernels in the 
sense of Carathéodory, and on the other hand of compact sets in the Hausdorff metric.

We continue in Chapter 2 with a description of the function $g_{\lambda, \mu}$ in a dynamical sense. At first we prove the existence of the attracting basin $\mathcal{A}_{\mu}$, followed by a discussion and some computations of the function $g_{\lambda, \mu}$ restricted as real functions. In Section 2.3 we describe the location of the critical points and values of $g_{\lambda, \mu}$ which serves to introduce the construction of the absorbing domains presented in Section 2.4. The construction of the invariant Jordan curves and the proof of the Structure Theorem can be found in Section 2.5. We finish that chapter by proving the property of Polynomial-Like Mapping in Section 2.6.

In Chapter 3 we are mainly concerned with convergences as $\mu$ tends to zero. Therefore we prove in Section 3.1 the non existence of wandering domains for $g_{\lambda}$ and present further results of this function needed in the sequel. In Section 3.2 we prove the existence of a sequence of attracting fixed points $z_{p, \mu}$ of $g_{\lambda, \mu}$ converging to an indifferent fixed point $z_{p}$ of $g_{\lambda}$. In Section 3.3 we establish separately the kernel convergence of the attracting basins $\mathcal{A}_{\mu}$ to the Baker domain $\mathcal{B}_{\lambda}$, and of the attracting basins $\mathcal{A}_{p, \mu}$ to the basin $\mathcal{D}_{p}$ in the case that $z_{p}$ is a parabolic fixed point. In Section 3.4 we show the convergence of the filled Julia sets and conclude the present work with the convergence of the Julia sets in the Hausdorff metric, that is, $\mathcal{J}\left(g_{\lambda, \mu}\right) \rightarrow \mathcal{J}\left(g_{\lambda}\right)$ as $\mu$ tends to zero. 


\section{Stories out of pictures}

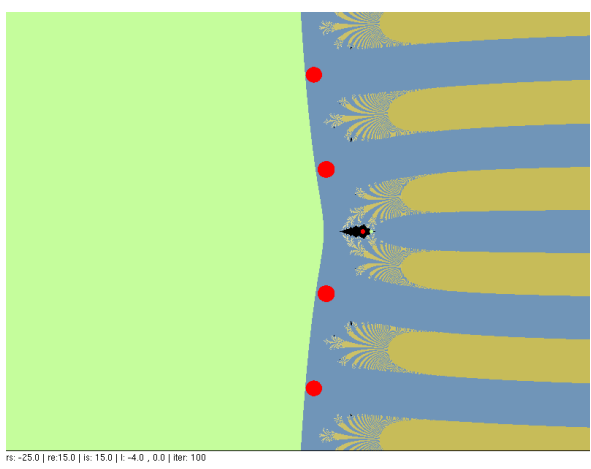

a1) $\lambda=-4$; Re $z \in(-25,15)$;

$\operatorname{Im} z \in(-15,15)$

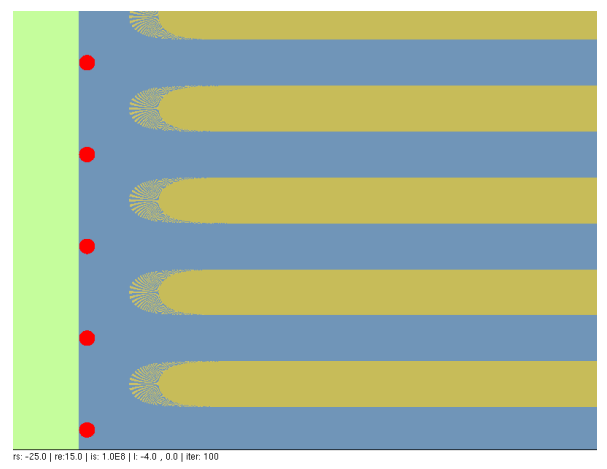

a2) $\lambda=-4 ; \operatorname{Re} z \in(-25,15)$; $\operatorname{Im} z \in\left(10^{8}-30,10^{8}\right)$

Figure 1: Fatou components of the function $g_{\lambda}$ : Baker domain $\mathcal{B}_{\lambda}$ and basin of attraction $\mathcal{A}_{0}$ close to the origin. $\mathcal{J}\left(g_{\lambda}\right)$ is drawn in brown.

In Figures 1 we see the Baker domain $\mathcal{B}_{\lambda}$ in blue. The absorbing domain $\mathcal{M}_{1 / 2}$ in drawn in light green and is contained in a left half-plane. The Julia set $\mathcal{J}\left(g_{\lambda}\right)$ is showed in light brown and a neighbourhood of each critical point is drawn in red. We see that for points with small imaginary part in a1) as for points with large imaginary part in a2) the critical points lie close to $\partial \mathcal{M}_{1 / 2}$. For the parameter $\lambda=-4 \in \mathbb{R}_{-}$the free critical point $c_{0}$ does not belong to $\mathcal{B}_{\lambda}$ but to the basin of attraction $\mathcal{A}_{0}$, which can be found in black close to the origin in a1).

Figures 2 b1) to d1) show both basins of attraction $\mathcal{A}_{\mu}$ and $\mathcal{A}_{0, \mu}$ for the same parameter $\lambda=-4 \in \mathbb{R}_{\text {- }}$ and different values of $\mu \in\left(0, \frac{1}{2}\right)$. The subsets of the absorbing domain $\mathcal{H}_{\eta, \rho}$ are showed in light green for $\mathcal{M}_{\rho}$ and dark green for $\mathcal{H}_{\eta}$. We appreciate that for $\mu=\frac{1}{10}$ which is not sufficiently small, the subdomain $\mathcal{M}_{\rho}$ is not large enough. The Julia set $\mathcal{J}\left(g_{\lambda, \mu}\right)$, drawn in light brown, exhibits a very chaotic behaviour for points with very large imaginary part in b2). However, as the value of $\mu$ decreases towards zero, the absorbing domain grows. For $\mu=\frac{1}{20}$ the dynamics are well controlled for points with small imaginary part and for points with large imaginary part the picture "looks better". For $\mu=\frac{1}{100}$ a larger number of critical points lie close to $\partial \mathcal{M}_{\rho}$, as it can be seen in $\mathrm{d} 2$ ).

In other words, as we take the limit $\mu \rightarrow 0$ the control over the critical values improves as there is a larger number of them lying in $\mathcal{M}_{\rho}$. The possibly existing Fatou components besides $\mathcal{A}_{\mu}$ and $\mathcal{A}_{0, \mu}$ shrink upwards and downwards to $\infty$ until they vanish in the limit. 


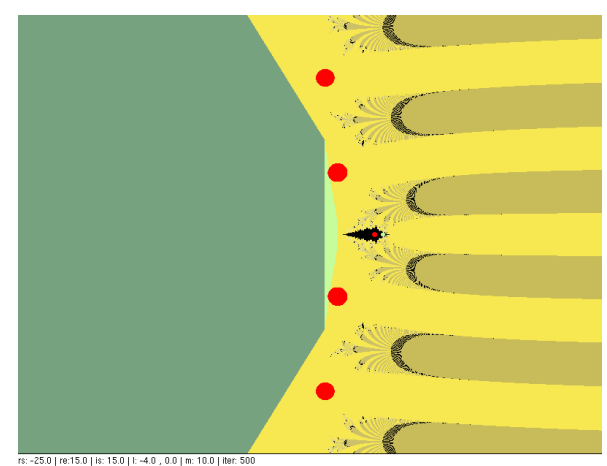

b1) $\lambda=-4 ; \mu=\frac{1}{10} ; \operatorname{Re} z \in(-25,15)$; $\operatorname{Im} z \in(-15,15)$

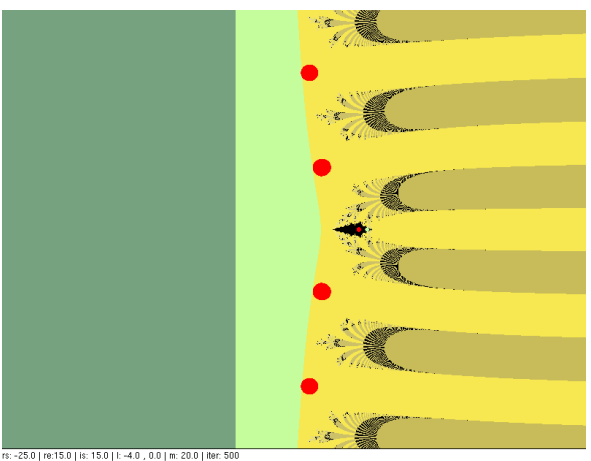

c1) $\lambda=-4 ; \mu=\frac{1}{20} ; \operatorname{Re} z \in(-25,15)$; $\operatorname{Im} z \in(-15,15)$

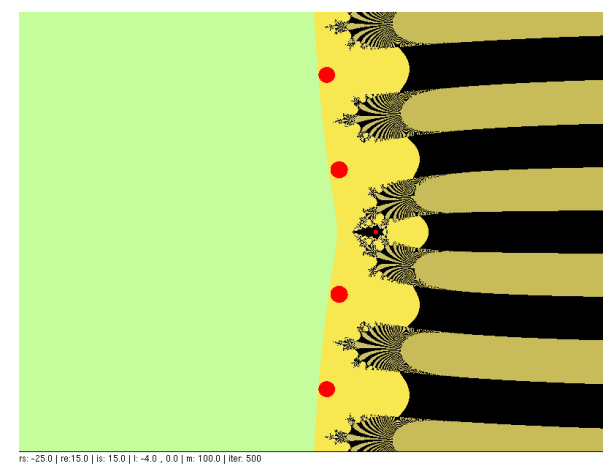

d1) $\lambda=-4 ; \mu=\frac{1}{100} ; \operatorname{Re} z \in(-25,15)$; $\operatorname{Im} z \in(-15,15)$

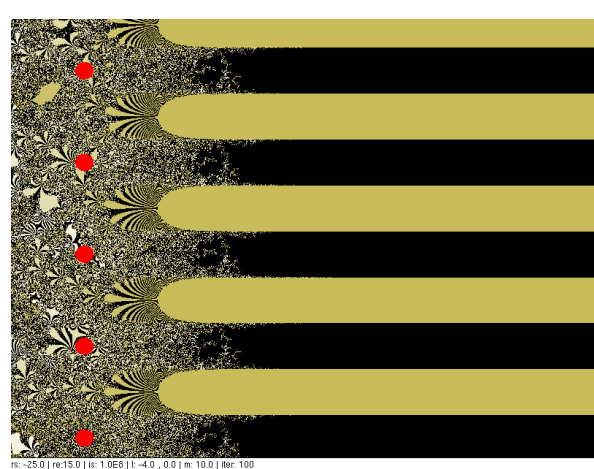

b2) $\lambda=-4 ; \mu=\frac{1}{10} ; \operatorname{Re} z \in(-25,15)$; $\operatorname{Im} z \in\left(10^{8}-30,10^{8}\right)$

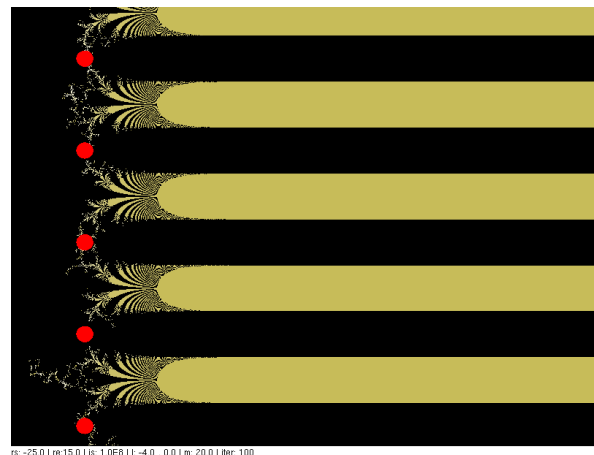

c2) $\lambda=-4 ; \mu=\frac{1}{20} ; \operatorname{Re} z \in(-25,15)$; $\operatorname{Im} z \in\left(10^{8}-30,10^{8}\right)$

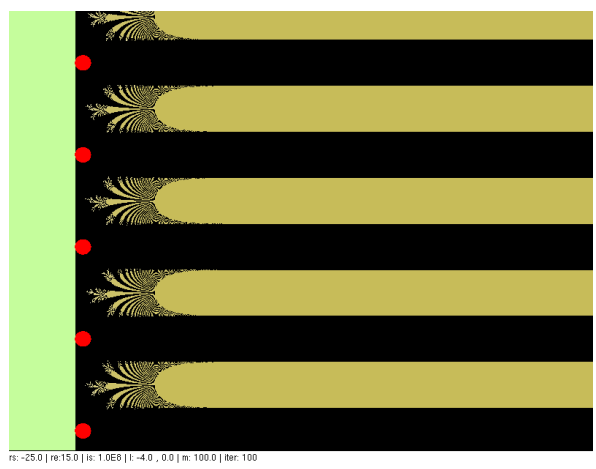

d2) $\lambda=-4 ; \mu=\frac{1}{100} ; \operatorname{Re} z \in(-25,15)$; $\operatorname{Im} z \in\left(10^{8}-30,10^{8}\right)$

Figure 2: Fatou components of the function $g_{\lambda, \mu}$ : Basin of attraction $\mathcal{A}_{\mu}$ and basin of attraction $\mathcal{A}_{0, \mu}$ close to the origin. $\mathcal{J}\left(g_{\lambda, \mu}\right)$ is drawn in brown. 
Contents 


\section{Preliminaries and known results}

During the present work we deal with the iteration of analytic functions of one complex variable, that is, with holomorphic functions. Therefore the area is also known as Holomorphic Dynamics. Even though we deal mainly with transcendental entire functions, we present in the following sections results which may apply as well to polynomials and rational functions or even to meromorphic functions. We mention it explicitly in case there is a distinction.

We begin by giving a short compendium of results in complex analysis, followed by some definitions and theorems in the theory of holomorphic dynamics. All theorems presented in this chapter are known results, therefore we state them without proof but giving explicit reference to the literature, if considered as necessary.

Some references for standard results on rational functions are Beardon's [10], Milnor's [51] or Carleson and Gamelin's [15] books. A book which considers both transcendental entire and meromorphic functions is due to Morosawa et. al. [54]. One of the first surveys dealing with meromorphic functions is due to Bergweiler [12]. Rippon [56] dedicated a survey to Baker domains, in honour to the trajectory of Prof. Noel Baker. Further important works about transcendental functions are [25, 26] or the classical references due to Fatou, Julia or Cremer are [17, 29, 30, 34, 35], among others. 


\subsection{Preliminaries from complex analysis}

Let $\mathbb{C}$ be the complex plane and $\widehat{\mathbb{C}}:=\mathbb{C} \cup\{\infty\}$ the Riemann sphere, that is, the onepoint compactification of the complex plane. During this chapter let $f: \mathbb{C} \rightarrow \mathbb{C}$ be a transcendental entire function, or $f: \widehat{\mathbb{C}} \rightarrow \widehat{\mathbb{C}}$ be a polynomial or a rational function and in the three cases $f$ is neither constant nor a linear function. We present some relevant results from complex analysis required for the definitions of the Fatou and Julia sets and further results in the area of holomorphic dynamics. Some of this results may apply as well for meromorphic functions. Even though, we restrict to assertions for entire functions which lie within the scope of this work.

A transcendental entire function can be defined as a function of a complex variable $z$ which is differentiable everywhere on the complex plane and it is not a polynomial. A different point of view on the definition of a transcendental entire function is that these functions can be represented as the limit of an everywhere-convergent Taylor series expansion for all finite $z$, that is, as

$$
f(z)=a_{0}+a_{1} z+a_{2} z^{2}+\ldots+a_{n} z^{n}+\ldots,
$$

where infinitely many of the coefficients $a_{n}$ are not vanishing. In particular, transcendental entire functions have an essential singularity at infinity.

The consequences of this representation are enormous. The first of this is that if $f(z)$ is any entire function which is never zero, then $f(z)$ is of the form $e^{g(z)}$, where $g(z)$ is also an entire (not necessarily transcendental) function.

We refer in the following to a simple closed curve as a Jordan curve and to a simple arc as a Jordan arc. Furthermore a domain $U$ is an open simply connected subset of $\mathbb{C}$ equipped with the euclidean metric. In the case that $U$ is consider as a subset of $\widehat{\mathbb{C}}$, then we mention it explicitly and consider $\widehat{\mathbb{C}}$ equipped with the chordal metric.

For any given entire function $f: \mathbb{C} \rightarrow \mathbb{C}$ we consider the sequence of iterates $\left\{f^{n}\right\}_{n \in \mathbb{N}}$, with $f^{n}=f \circ f^{n-1}$ for $n \geq 1$ and $f^{0}=\mathrm{id}$. Roughly speaking, the Fatou set of $f$ is set of points in the complex plane where the sequence of iterates are locally stable and the Julia set is its complement in the Riemann sphere. In order to give a precise definition for this, we introduce the concept of a normal family.

Consider a family of holomorphic functions $\left\{f_{j}\right\}_{j \in I}$ for some index set $I$. Then $\left\{f_{j}\right\}$ is said to be a normal family on a domain $U$ if every sequence of $f_{j}$ 's contains either a subsequence that converges uniformly on every compact subset of $U$ to a 
holomorphic function $g: U \rightarrow \mathbb{C}$, or a subsequence that converges uniformly on every compact subset of $U$ to $\infty$.

A useful tool used to determine whether a family of functions is normal, or which is the largest domain where the family does not fail to be normal, is given by Montel's Theorem.

Theorem 1.1. Let $\left\{f_{j}\right\}$ be a family of holomorphic functions $f_{j}: \mathbb{C} \rightarrow \mathbb{C}$. Assume there exist two distinct values $z_{1}$ and $z_{2}$ in $U \subseteq \mathbb{C}$ that are never taken by the functions $f_{j}$, that is $f_{j}(z) \in \mathbb{C} \backslash\left\{z_{1}, z_{2}\right\}$ for every $z \in U$ and every $f_{j}$. Then $\left\{f_{j}\right\}$ is a normal family on $U$.

If the functions $f_{j}$ satisfy $f_{j}: \widehat{\mathbb{C}} \rightarrow \widehat{\mathbb{C}}$, then they should omit three distinct points in order to form a normal family. In the present work we usually take for such a family the sequence of iterates $f^{n}$, with $n \in \mathbb{N}$, of a given entire function $f$. Another important result in the theory of complex analysis is the Picard's little theorem.

Theorem 1.2. If $f$ is a transcendental entire function, then the equation $f(z)=w$ has infinitely many roots for any $w \in \mathbb{C}$, except for at most one value.

There are several versions of this theorem, some of them stated by Picard himself. Another formulation says that an entire function with more than one finite omitted value reduces to a constant. But in the context of this work, the version stated above is the one which at the best reflects its application. In the frame of this theorem we call a point $w \in \mathbb{C}$ Picard omitted value or exceptional point if and only if it has at most a finite number of preimages.

The following theorem is in fact a corollary to Cauchy's integral formula for meromorphic functions and the argument principle. However, it is best known as Rouché's Theorem.

Theorem 1.3. Let $\gamma$ be a Jordan curve homologous to zero in a domain U. Assume $f$ and $g$ are holomorphic in $U$ and satisfy the inequality $|f(z)-g(z)|<|f(z)|$ on $\gamma$. Then $f(z)$ and $g(z)$ have the same number of zeros enclosed by $\gamma$.

A theorem due to Weierstrass states that given a sequence of holomorphic functions $\left\{f_{n}(z)\right\}_{n \in \mathbb{N}}$ in a domain $U$ converging uniformly on every compact subset of $U$ to a limit function $f(z)$, then $f(z)$ is holomorphic in $U$ as well. Moreover, $f_{n}^{\prime}(z)$ converges uniformly to $f^{\prime}(z)$ on every compact subset of $U$.

One last result from the complex analysis which we mention is the RiemannHurwitz Formula for domains. 
Lemma 1.4. Let $D_{1}$ and $D_{2}$ be domains in $\widehat{\mathbb{C}}$ whose boundaries consists of a finite number of Jordan curves. Let $f(z)$ be a proper holomorphic map of $D_{1}$ onto $D_{2}$. Then:

i) Every $z \in D_{2}$ has the same number $k$ of preimages counted with multiplicity.

ii) Denote by $N$ the number of critical points of $f$ in $D_{1}$ counted with multiplicity. Then

$$
\left(2-d_{1}\right)=k\left(2-d_{2}\right)-N
$$

where $d_{j}$ is the number the boundary components of $D_{j}$.

In particular, if both the $D_{j}$ are simply connected, then $f$ has at most $k-1$ critical points.

\subsection{Singular values}

The set of singular values plays an important role in the theory of holomorphic dynamics, as we shall see below in Section 1.6. A point $\zeta \in \mathbb{C}$ is a singular value if for every neighbourhood $V$ of $\zeta$, there exists a branch of the inverse of $f$ that is not holomorphic in $V$. We denote the set of singular values by $\operatorname{sing}\left(f^{-1}\right)$ and its forward iterates, called the post-singular values, by $P(f):=\bigcup_{n=0}^{\infty} f^{n}\left(\operatorname{sing}\left(f^{-1}\right)\right)$.

For an entire function $\operatorname{sing}\left(f^{-1}\right)$ consists of critical values and finite asymptotic values. In the case that $f$ has infinitely many critical and asymptotic values, then their limit points belong as well to $\operatorname{sing}\left(f^{-1}\right)$.

We say that $\zeta \in \mathbb{C}$ is a critical value of an entire function $f$ if it is the image of a critical point $z$, it means $\zeta=f(z)$, where $z$ satisfies the condition that $f^{\prime}(z)=0$.

A point $\zeta \in \mathbb{C}$ is called a finite asymptotic value of $f$ if there exists a parametrized curve $\gamma:[0, \infty) \rightarrow \mathbb{C}$ satisfying $\lim _{t \rightarrow \infty} \gamma(t)=\infty$ and $\lim _{t \rightarrow \infty} f(y(t))=\zeta$.

The set of singular values of a polynomial or a rational function consists of the critical values, only, and there is always a finite number of them. In contrast to this, transcendental functions may have infinitely many singular values. A transcendental entire function that has only finitely many singular values is called critically finite. 


\subsection{Fixed and periodic points}

Consider now a fixed point of a function, that is, a point $z$ such that $f(z)=z$. In a more general way, a periodic point satisfies $f^{p}\left(z_{0}\right)=z_{0}$ for some (minimal) $p \geq 1$. In case that $z_{0}$ is a periodic point of period $p$, we define the cycle of $z_{0}$ as the set $\left\{z_{0}, f\left(z_{0}\right), \ldots, f^{p-1}\left(z_{0}\right)\right\}$.

A fixed point $z_{0}$ is called attracting if $\left|f^{\prime}\left(z_{0}\right)\right|<1$, repelling if $\left|f^{\prime}\left(z_{0}\right)\right|>1$ and indifferent if $\left|f^{\prime}\left(z_{0}\right)\right|=1$. In the special case when $f^{\prime}\left(z_{0}\right)=0$, $z_{0}$ is called $s u$ perattracting fixed point. These definitions hold as well for periodic points by considering the derivative $\left(f^{p}\right)^{\prime}$ instead of $f^{\prime}$. The derivative $\left(f^{p}\right)^{\prime}\left(z_{0}\right)$ is called multiplier of $z_{0}$ and it has the same value for all points in the cycle of $z_{0}$.

If $\left|f^{\prime}\left(z_{0}\right)\right|=1$, then we may write $f^{\prime}\left(z_{0}\right)=e^{2 \pi i \theta_{0}}$ for some $\theta_{0} \in[0,1)$. Then the point $z_{0}$ is called rationally indifferent or parabolic if $\theta_{0}$ is a rational number, and irrationally indifferent otherwise.

The forward orbit of a point $z$ is defined as the set $O^{+}(z):=\bigcup_{n \in \mathbb{N}} f^{n}(z)$. Notice that this set is finite for any periodic or preperiodic point, where the latter is a point $z$ such that $f^{n}(z)=f^{m}(z)$ for some $n, m \in \mathbb{N}$ with $m<n$. The backward orbit of $z$ is the set $O^{-}(z):=\left\{w \in \mathbb{C}: f^{n}(w)=z\right.$ for some $\left.n \in \mathbb{N}\right\}$. It is not difficult to see that a rational function has periodic points of any period $p \geq 1$. Even though this question may not be trivial for entire functions, this result was firstly prove by Fatou and generalised by Rosenbloom in the following form.

Theorem 1.5. A transcendental entire function has infinitely many periodic points of period $p$ for all $p \geq 2$.

It is worth to mention that an irrational indifferent periodic point $z_{0}$ may lie in the Julia set as well as in the Fatou set, while parabolic periodic points always lie in the Julia set. It holds that $z_{0}$ lies in the Fatou set if and only if $z_{0}$ is linearisable. A irrational indifferent point $z_{0}$ which lies in $J(f)$ is called Cremer point and satisfies the following result.

Theorem 1.6. If $z$ is a Cremer point of an entire function $f$, then $z$ is contained in the set of accumulation points of $P(f)$.

\subsection{Fatou and Julia sets}

The field of holomorphic dynamics deals with the iteration of functions in the complex plane or in the Riemann sphere. We divide the complex plane into two main sets according to the behaviour under the iterates of $f$ on a given point, 
that is, under the sequence $\left\{f^{n}\right\}_{n \in \mathbb{N}}$, where $f^{n}=f \circ f^{n-1}$ and $f^{0}$ is the identity function.

Consider $f$ to be a non linear entire function or a rational map of degree at least two. We define the Fatou set of $f, \mathcal{F}(f)$, as the set of points $z \in \mathbb{C}$ where the sequence of iterates $\left\{f^{n}\right\}_{n \in \mathbb{N}}$ is well-defined and forms a normal family in some neighbourhood of $z$. The Julia set is defined as its complement in the Riemann sphere, that is, $\mathcal{J}(f):=\widehat{\mathbb{C}} \backslash \mathcal{F}(f)$. It implies by definition, that $\infty \in \mathcal{J}(f)$ for any transcendental entire function, since $\infty$ is the only essential singularity for such functions.

Some properties of the Fatou and Julia sets are:

- $\mathcal{F}(f)$ is an open set and $\mathcal{J}(f)$ is a closed set.

- $\mathcal{F}(f)$ and $\mathcal{J}(f)$ are completely invariant.

- $\mathcal{J}(f)$ is a perfect set; that is, $\mathcal{J}(f)$ is closed, non-empty and contains no isolated points.

- For every positive $n \in \mathbb{N}, \mathcal{J}\left(f^{n}\right)=\mathcal{J}(f)$ and $\mathcal{F}\left(f^{n}\right)=\mathcal{F}(f)$.

- Either $\mathcal{J}(f)=\widehat{\mathbb{C}}$ or $\mathcal{J}(f)$ has empty interior.

- If $z_{0} \in \mathcal{J}(f)$ is not an Picard omitted value, then $\mathcal{J}(f)=\overline{O^{-}\left(z_{0}\right)}$.

For any $z_{0} \in \mathcal{J}(z)$ and $U$ a neighbourhood of $z_{0}$, it follows by Montel's Theorem that $\mathbb{C} \backslash \cup_{n=1}^{\infty} f^{n}(U)$ consists of at most two points. This reflects the sensitivity of the Julia set to initial conditions, one of the chaotic characteristics of this set.

Note that all repelling periodic points lie in the Julia set. Moreover, $\mathcal{J}(f)$ equals the closure of set of repelling periodic points of $f$. Rationally indifferent periodic points lie as well in the Julia set, since there have attracting directions and repelling directions. Therefore, we cannot have uniform convergence of the iterates in any neighbourhood of these points. It is clear in the same way, that attracting periodic points lie in the Fatou set.

The topological structure of the Julia set may be very intricate. Devaney and Krych studied in [19] the exponential family $E_{\lambda}(z)=\lambda e^{z}$ and found out that for parameters $0<\lambda<1 / e$ the Julia set $J\left(E_{\lambda}\right)$ consists of a Cantor set of curves called Cantor bouquet. Since then there are several examples of functions having Cantor bouquets. For example, Devaney and Tangerman showed the existence of Cantor Bouquets for some class of critically finite entire functions; see [21]. Aarts and Overteegen extended in [1] the notion of Cantor bouquets, also called straight 
brush, for some class of hyperbolic entire functions, that is functions for which $\operatorname{sing}\left(f^{-1}\right)$ is relatively compact in $\mathcal{F}(f)$.

Moreover, Misiurewicz gave in [52] a straightforward proof that the Julia set of $E(z)=e^{z}$ equals the whole plane. This holds for every $E_{\lambda}(z)=\lambda e^{z}$ with $\lambda>1 / e$ as it was showen by Devaney in [18. Devaney gave as well a description of an invariant compact set, an indecomposable continuum, for parameters $\lambda>1 / e$ and further properties of the Julia set for the exponential family. These ideas reflect the complexity of the Julia set. We present further characteristics of the Julia set which are directly related to Baker and wandering domains in Section 1.7.

A last result beautiful for its simplicity and strength is mentioned by Bergweiler in [12. It states that if $f$ is a transcendental entire function, then $\mathcal{J}(f)$ does not contain isolated Jordan arcs. Its proof lies mainly on the fact that $\mathcal{J}(f)=\overline{O^{-}\left(z_{0}\right)}$ for some $z_{0} \in \mathcal{J}(f)$.

\subsection{Fatou components}

A connected component $U \subset \mathbb{C}$ of $\mathcal{F}(f)$ is called a Fatou component. If there exists a minimal $p \in \mathbb{N}^{*}$ such that $f^{p}(U) \subset U$ then $U$ is called a periodic Fatou component of period $p$ and we denote it by $U_{p}$. A component $U$ is called preperiodic if there exist $p>q \geq 0$ such that $U_{p} \subset U_{q}$. Analog to periodic points, we call $\left\{U, U_{1}, \ldots, U_{p-1}\right\}$ a (periodic) cycle of components. If $p=1$, that is, $f(U) \subset U$, then $U$ is called invariant. Most of these components have a direct relation with fixed or periodic points of $f$.

There are four possible types of periodic Fatou components that can occur for entire functions or rational functions. We introduce as well the notation we use in the present work for each component.

- If $f$ has an attracting or superattracting periodic point $z_{0}$ of period $p$, then let $\mathcal{A}$ consists of all points $z \in \mathbb{C}$ such that $f^{n p}(z) \rightarrow z_{0}$ as $n \rightarrow \infty$. $\mathcal{A}$ is generally called attracting basin of $z_{0}$ or in particular Böttcher domain if $z_{0}$ is superattracting, or Schröder domain if $z_{0}$ is attracting. The immediate attracting basin $\mathcal{A}^{*}$ is the component containing the periodic point $z_{0}$. It holds that $\partial \mathcal{A}=\mathcal{J}$ and each component of $\mathcal{A}$ is a preperiodic Fatou component.

- Given a parabolic periodic point $z_{0}$, we call a parabolic basin $\mathcal{D}$ the set of points $z \in \mathbb{C}$ such that $f^{n p}(z) \rightarrow z_{0}$ as $n \rightarrow \infty$. In this case $z_{0} \in \partial \mathcal{D}$ and the immediate parabolic basin is the set $\mathcal{D}^{*}=\bigcup_{j=1}^{m} \mathcal{D}_{j}$ such that $\bigcap_{j=1}^{m} \partial \mathcal{D}_{j}=z_{0}$. 
The component $\mathcal{D}$ is also called Leau domain. It holds again that $\partial \mathcal{D}=\mathcal{J}$ and each component of $\mathcal{D}$ is a preperiodic Fatou component.

- A Siegel disc $\mathcal{S}$ is a periodic Fatou component on which $f^{p}: \mathcal{S} \rightarrow \mathcal{S}$ is analytically conjugate to an irrational rotation of the open unit disc onto itself. $S$ contains an irrational indifferent fixed point which is called the centre of the Siegel disc.

- A Herman Ring $\mathcal{H}$ is a periodic Fatou component on which $f^{p}: \mathcal{H} \rightarrow \mathcal{H}$ is analytically conjugated to an irrational rotation of an annulus $A=\{z \in \mathbb{C}$ : $1<|z|<r\}$, for some $r>1$, onto itself.

- If $f$ is a transcendental function, then a Baker domain or domain at $\infty$ is a Fatou component $\mathcal{B}$ with $f^{n p}(z) \rightarrow \infty$ as $n \rightarrow \infty$ for all $z \in \mathcal{B}$.

For a meromorphic function, the definition of a Baker domain extends to those points $z$ where $f^{n p}(z) \rightarrow z_{0}$, with $z_{0} \in \partial \mathcal{B}$ and $f^{p}\left(z_{0}\right)$ is not defined. For entire functions the only singularity lies at $\infty$, hence the definition of a Baker domain reduces to the case mentioned above.

Herman Rings may appear only for rational functions or transcendental meromorphic function, using the maximum principle, and we mention them here for completeness only. Moreover Baker domains occur only for transcendental entire or transcendental meromorphic functions.

Furthermore, transcendental entire (and meromorphic) functions may have a component $W$ that is neither periodic nor eventually periodic.

- A wandering domain $\mathcal{W}$ is a domain where the sequence of iterates is never preperiodic, i.e. $f^{n}(\mathcal{W}) \cap f^{m}(\mathcal{W})=\emptyset$ whenever $n \neq m$ for $n, m \in \mathbb{N}$.

One of the fundamental theorems in holomorphic dynamics is due to Sullivan.

Theorem 1.7. Let $f$ be a rational function. Then $\mathcal{F}(f)$ has no wandering domains.

It was already proved by Fatou in [29] that the connectivity of an invariant component of $\mathcal{F}(f)$ takes one of the values 1,2 or $\infty$ if $f$ is a rational function. It implies the same result for periodic components but not for preperiodic components. It was proved by Baker, Kotus und Lü in [6] that preperiodic components may take any connectivity if $f$ is a rational or a meromorphic function.

In contrast to this Baker proved that for polynomials the connectivity of any component, periodic or preperiodic, is 1 or 2 , where 2 only occurs for Herman rings. His results extends to entire functions as follows. 
Theorem 1.8. If $f$ is a transcendental entire function, then any preperiodic component is simply connected.

In other words, multiply connected components are necessarily wandering domains. Moreover, Kisaka and Shishikura proved in [38] that there does not exist any entire function with multiply connected components of finite connectivity, that is, wandering domains must be infinitely connected.

In the present work we concern mainly with the convergence of functions and their Fatou and Julia sets. A first result in this direction is called persistence of attracting (resp. repelling) cycles. The reader can find a proof of the following result in [4], which is mainly based on the well-known result of Hirsch and Smale (persistence under $\mathcal{C}^{1}$ perturbations) and on Rouché's Theorem.

Theorem 1.9. Let $f$ be a transcendental entire function and let $\left\{f_{n}\right\}$ be a sequence of entire functions converging to $f$ uniformly on compact subsets of $\mathbb{C}$. Let $z_{0}$ be an attracting (resp. repelling) fixed point of $f$. Then

- There is an $N \in \mathbb{N}$ such that $f_{n}$ has an attracting (resp. repelling) fixed point $z_{n}$ for all $n \geq N$. Moreover, $z_{n}$ converges to $z_{0}$ and the orbit $O^{+}\left(z_{0}\right)$ converges to $\mathrm{O}^{+}\left(z_{0}\right)$ as compact sets with respect to the Hausdorff metric.

- Let $\mathcal{A}\left(z_{0}\right)$ be the basin of attraction of $z_{0}$. Then for every compact $K \subset \mathcal{A}\left(z_{0}\right)$ there is an $N \in \mathbb{N}$, such that $K \subset \mathcal{A}\left(z_{n}\right)$ for all $n>N$, where $\mathcal{A}\left(z_{n}\right)$ is the basin attraction of $z_{n}$ converging to $z_{0}$.

The result holds also for attracting (rep.) periodic cycles and their orbits. See Section 1.9 for a definition of convergence in the Hausdorff metric.

\subsection{Singular values and Fatou components}

The importance of the set of singular values mainly lies in its relation with the Fatou components. This relation was already established by Fatou in the early 20's who proved that any basin of attraction must contain at least one singular value in its immediate basin. Fatou proved in [29] the following result for rational functions but his proof extends to meromorphic functions, as mentioned by Bergweiler in [12].

Theorem 1.10. Let $f$ be an entire function, and let $C=\left\{U_{0}, U_{1}, \ldots, U_{p-1}\right\}$ a periodic cycle of components of $\mathcal{F}(f)$.

- If $C$ is a cycle of attracting basins or parabolic basins, then $U_{j} \cap \operatorname{sing}\left(f^{-1}\right) \neq \emptyset$ for some $j \in\{0,1, \ldots p-1\}$. 
- If $C$ is a cycle of Siegel discs, then $\partial U_{j} \subset \overline{O^{+}\left(\operatorname{sing}\left(f^{-1}\right)\right)}$ for all $j \in$ $\{0,1, \ldots p-1\}$.

It follows directly that if $f$ has $n$ singular values, then $f$ can have at most $n$ attracting and parabolic basins. Moreover, if $f$ is a rational functions, it is possible to estimate in general the number of Fatou components in terms of its degree. The first estimates were done by Fatou and Sullivan and sharpened by Shishikura, [58]. If $f$ is a rational function of degree $d$, then the number of cycles of immediate attracting basins, parabolic basins, and Siegel discs plus twice the number of Herman rings does not exceed $2 d-2$.

There is not direct relation between singular values and Baker domains. In fact there are examples of univalent Baker domains as well as examples of Baker domains with infinitely many singular values, as we present more in detail in the following section. Nevertheless it is possible to exclude the existence of Baker domains regarding the distribution of the set of singular values. Eremenko and Lyubich [26] proved the following theorem.

Theorem 1.11. Let $f$ be a transcendental entire function such that $\operatorname{sing}\left(f^{-1}\right)$ is a bounded set. Then $f$ has no Baker domains.

A generalisation of this result states that $\infty$ is in the derived set of $P(f)$ if $f$ has a cycle of Baker domains. Hence it follows as a corollary that if $f$ has finitely many singular values, then $f$ has no Baker domains. The following result is an analogue result about wandering domains

Theorem 1.12. Let $f$ be a transcendental entire function with finitely many singular values. Then $f$ has no wandering domains.

\subsection{On Baker domains and wandering domains}

We already mentioned above some results concerning wandering domains and Baker domains. Here we dedicate a section to these Fatou components since they play a central role in the present work.

The first example of a transcendental entire function having a Baker domain was given by Fatou in [30]. Fatou considered the function $f(z)=z+1+e^{-z}$ and proved that for points $z$ with $\operatorname{Re} z>0$, the iterates under $f$ tend to infinity. In this case, the domain $\mathcal{B}$ is invariant and contains the right half-plane $\mathbb{H}_{+}$and infinitely many singular values which lie on $i \mathbb{R}$.

As we mention before Baker domains may or may not contain singular values. In the latter case, we called them univalent Baker domains. Bergweiler gave an 
example of an univalent Baker domain in [11. He proved via logarithmic lift that the function $f(z)=2 z+\log 2-2+e^{-z}$ has a Baker domain $\mathcal{B}$ and that $\partial \mathcal{B}$ is a Jordan curve in $\widehat{\mathbb{C}}$.

An example of a periodic cycle of Baker domains for transcendental entire functions was given by Rippon and Stallard in [57]. Firstly they showed that the function $f(z)=a z\left(1+e^{z^{-p}}\right)$, with $a>1, p \in \mathbb{N}$, has $p$ invariant Baker domains, one in each sector $\{z \in \mathbb{C}:|\arg z-2 k \pi / p|<\pi / p\}, k \in\{0,1, \ldots, p-1\}$. Secondly, it follows that the function $g(z)=e^{2 \pi i / p} f(z)$ has a $p$-cycle of Baker domains.

In general, the boundary of a Baker domain may have a complicated topological structure. Therefore we introduce the concept of an absorbing domain for $f$, which is a domain $D \subset \mathcal{B}$ satisfying the following conditions:

- $D$ is simply connected.

- $f(D) \subset D$.

- For every compact set $K \subset \mathcal{B}$ there exists an $n \in \mathbb{N}$ such that $f^{n}(K) \subset D$.

In Fatou's example above, an absorbing domain of $\mathcal{B}$ is the half-plane $\mathbb{H}_{+}$. This definition is mostly used for Baker domains, but it can be also applied for basins of attraction or even for domains in the attracting directions of parabolic fixed points. In the latter case, these domains are usually called attracting petals of a parabolic fixed point. Notice that an absorbing domain need not be unique.

The following theorem is result concerning Baker and the singular values of the function and it was proved by Bergweiler in [11, Lemma 3].

Theorem 1.13. Let $f$ be a transcendental entire function with an invariant Baker domain $\mathcal{B}$. Suppose that $K \subset \mathcal{B}$ is compact and that $\tau>1$. Then there exists $n_{0}$ such that

$$
D\left(f^{n}(z), \tau \operatorname{dist}\left(f^{n}(z), \partial \mathcal{B}\right)\right) \cap P(f) \neq \emptyset
$$

for all $z \in K$ and $n \geq n_{0}$.

Notice that the result applies as well to any univalent Baker domain $\mathcal{B}$ and in this case implies that the singular values are (always) close to the boundary of $\mathcal{B}$.

We present now a classification of the types of Baker domains of a function $f$ due to König; see [40]. Let $\psi$ be a Riemann mapping from a simply connected domain $\mathbb{D}$ onto $U$. Then the function $g:=\psi^{-1} \circ f \circ \psi$ is a self-mapping of $\mathbb{D}$. Using Denjoy-Wolff Theorem, there is a point $\zeta \in \overline{\mathbb{D}}$ such that $g^{n}(z) \rightarrow \zeta$ as $n \rightarrow \infty$ for every $z \in \mathbb{D}$. The point $\zeta$ is called the Denjoy-Wolff point. In [16] Cowen proved 
that $g$ is conjugated to a Möbius transformation $T$ under certain conditions and classified the self-maps $g$ of $\mathbb{D}$ for any domain $U$ using the following definition.

Let $f$ be an entire function and $U$ a domain. The triple $(D, \phi, T)$ is called a conformal conjugacy of $f$ in $U$ if:

- $D \subset U$ is an absorbing domain for $f$.

- $\phi: U \rightarrow \Omega \subset\left\{\mathbb{C}, \mathbb{H}_{+}\right\}$is analytic and univalent in $D$.

- $T: \Omega \rightarrow \Omega$ is a Möbius transformation, and $\Phi(D)$ is an absorbing domain for $T$.

- $\phi(f(z))=T(\phi(z))$, for $z \in U$.

Cowen proved that if the Denjoy-Wolff point $\zeta$ lies in $\partial \mathbb{D}$, then the mapping $g$ always has a conformal conjugacy. If $f$ is an entire function and $\mathcal{B}$ is a Baker domain, then $\mathcal{B}$ is simply connected and the fact that $f^{n} \rightarrow \infty$ in $\mathcal{B}$ implies that $\zeta \in \partial \mathbb{D}$. Based on Cowen's classification, König introduced a notation to classify the Baker domains as follows.

Theorem 1.14. Let $f$ be an entire function with a Baker domain $\mathcal{B}$. Then there exists a conformal conjugacy of $f$ in $\mathcal{B}$, which is exactly one of the following.

i) $T(z)=z+1$ and $\Omega=\mathbb{C}$.

ii) $T(z)=z \pm i$ and $\Omega=\mathbb{H}_{+}$.

iii) $T(z)=a z$, for $a>1$, and $\Omega=\mathbb{H}_{+}$.

Case i) is called parabolic of type I, case ii) is called parabolic of type II and case iii) is called hyperbolic.

König generalised this classification to meromorphic functions with finitely many poles and gave further geometrical criteria in [16]. There exists as well a classification for univalent Baker domains due to Barański and Fagella; see [9]. Fagella and Henriksen extended the latter classification in [28] to Baker domains $\mathcal{B}$ where $\left.f\right|_{\mathcal{B}}$ is a proper map. In these cases the results lie beyond the scope of this work, hence we omit them only including their reference.

Concerning the boundary of Baker domains, we present some results which we use in the following chapters. Baker and J. Weinreich proved in $[8]$ for any unbounded invariant Fatou component $U$ of an entire function that if $\partial U$ is a Jordan curve, then $U$ must be a Baker domain and $\left.f\right|_{U}$ must be univalent.

Consider again the Riemann mapping $\psi: \mathbb{D} \rightarrow U$ for an unbounded component $U$ and define the radial limit set as $\Theta:=\left\{e^{i \theta}: \psi\left(r e^{i \theta}\right) \rightarrow \infty\right.$ as $\left.r \rightarrow 1\right\}$. This set 
was introduced by Kisaka in [37] to study the connectivity of the Julia set and to proved the following theorem.

Theorem 1.15. Let $f$ be a transcendental entire function. Then $\mathcal{J}(f)$ is connected in $\widehat{\mathbb{C}}$ if and only if $f$ has no multiply connected wandering domains.

In particular, for a Baker domain $\mathcal{B}$ the set $\Theta$ is not empty. Baker and Domínguez proved in [7] the following result.

Theorem 1.16. Let $\mathcal{B}$ be an invariant Baker domain of an entire functions $f$ such that $\left.f\right|_{\mathcal{B}}$ is not univalent. Then $\bar{\Theta}$ contains a perfect set.

The latter results imply that $\infty$ has infinitely many accesses from within $\mathcal{B}$, since every point in $\Theta$ corresponds to a different access and $\Theta$ is dense in $\partial \mathbb{D}$. Hence $\partial \mathcal{B}$ has infinitely many components, that is $\mathcal{J}(f) \backslash \infty$ is disconnected. This result is in accordance with the concept of Cantor bouquets mentioned above in Section 1.4. For a larger list of examples and more detailed properties of Baker domains we refer the reader to the survey by Rippon in [56] or to Morosawa et al.'s book, [54].

Finally, we mention that for most points $z$ in a Baker domain $|f|$ is not more than a multiple of $|z|$ and that $f$ growths slowly. This was proved by Baker in [5] for entire functions having an invariant Baker domain.

Theorem 1.17. Let $\mathcal{B}$ be an invariant Baker domain of an entire function $f$. Then

- For any compact subset $K \subset \mathcal{B}$ there exist positive constant $C$ and $n_{0}$ such that $\left|f^{n}\left(z^{\prime}\right)\right| \leq C\left|f^{n}(z)\right|$ for $z, z^{\prime} \in K$ and $n \geq n_{0}$.

- For all $z \in \mathcal{B}, \ln \left|f^{n}(z)\right|=\mathcal{O}(n)$ as $n \rightarrow \infty$, where the constant $\mathcal{O}(n)$ depends on $z$.

- For any $z_{0} \in \mathcal{B}$ and a path $\gamma=\bigcup_{n=0}^{\infty} f^{n}\left(\gamma_{0}\right)$ where $\gamma_{0} \subset \mathcal{B}$ joins $z_{0}$ to $f\left(z_{0}\right)$ and $0 \notin \gamma$, there exists a positive constant $C$ such that

$$
\frac{1}{C}|z| \leq|f(z)| \leq C|z| \text { for } z \in \gamma
$$

The theorem was extended by Rippon in [55] for the more general case if $f$ is a meromorphic function having a $p$-cycle of Baker domains.

The first example of an entire function with a wandering domain was given by Baker in 1976; see [4, which was an example of a multiply connected wandering domain. 
A further example of a simply connected wandering domain constructed in a more elementary way was obtained via Newton's method by Herman in 1984; see [32]. Consider the function $h(z)=e^{z}-1$ and $g(z)=z-\frac{h^{\prime}(z)}{h(z)}=z-1+e^{-z}$, its Newton's method. For every $k \in \mathbb{Z}$ the point $z_{k}=2 \pi k i$ is a superattracting fixed point of $g$. Denote by $U_{k}$ the Fatou component containing $z_{k}$, that is, its immediate basin of attraction. It can be proved that $U_{k}$ is simply connected for every $k$. Finally consider the function $f(z)=g(z)+2 \pi i=z-1+e^{-z}+2 \pi i$. It holds that $\mathcal{F}(f)=\mathcal{F}(g)$ and that $f\left(U_{k}\right)=U_{k+1}$. Hence $\mathcal{F}(g)$ has a wandering domain.

It is well known that if $f$ is an entire function and $\mathcal{W}$ is a wandering domain of $f$, then all finite limit functions of $\left.f^{n}\right|_{\mathcal{W}}$ are constant. Fatou was aware of this result in 1920, [29], and Cremer proved it in 1936 as well, [17]. Baker proved that constant limit functions in any Fatou components are contained in $\overline{P(f)} \cup \infty$, [2]. These results imply the following theorem proved by Bergweiler et al. in [14] using elementary methods of complex analysis. Denote by $P(f)^{\prime}$ the derived set of $P(f)$, that is, the set of finite limit points of $P(f)=O^{+}\left(\operatorname{sing}\left(f^{-1}\right)\right)$.

Theorem 1.18. Let $f$ be an entire function and let $\mathcal{W}$ be a wandering domain of $f$. Then all limit functions of $\left\{\left.f\right|_{\mathcal{W}}\right\}$ are contained in $P(f)^{\prime} \cup \infty$.

This theorem enables to prove that certain functions do not have wandering domains, as we do it for a large set of parameters of the family of functions $g_{\lambda}$ in Section 3.1.

\subsection{On the polynomial-like mappings}

If we study a family of functions that depends analytically on a parameter, it is common to find copies of the Mandelbrot set in the parameter plane as we attempt to classify values of the parameter depending on the dynamical properties of the function. One way to explain this is, that for such families of functions there exist parameters for which the function behaves (locally) like a polynomial of degree 2 under iterates. The knowledge of polynomial-like mappings was developed by Douady and Hubbard in the middle 80's in order to give a precise meaning of "behaving like"; see [23].

This behaviour is not intrinsic to rational maps, but can also be found in families of transcendental entire functions, as long as there is some analytical dependence on the parameter. McMullen prove this for any holomorphic family of rational maps and called it "Universality of the Mandelbrot set", [50]. However, the universality is still not proved in general for families of transcendental entire functions. Therefore in Section 2.6 we apply this notion to the family of functions presented during 
this work. In general the concept is extended to the behaviour of a polynomial of degree $d$ and we present here the definition and some results.

Let $U$ and $V$ be bounded simply connected domains such that $\bar{U} \subset V$. A polynomial-like mapping of degree $d$ is a the triple $(f, U, V)$, where $f: U \rightarrow V$ is a holomorphic proper mapping of degree $d$.

If $(f, U, V)$ is a polynomial-like mapping of degree $d \geq 2$, we define the filled Julia set $K_{f}$ by setting

$$
K_{f}:=\left\{z \in \mathbb{C}: f^{n}(z) \in U \text { for every } n>0\right\} .
$$

The Straightening Theorem allows us to derive various properties from polynomials to the polynomial-like mappings of the same degree.

Theorem 1.19. Let $(f, U, V)$ be a polynomial-like mapping of degree $d \geq 2$. Then $f$ is hybrid equivalent to a polynomial $P$ of degree $d$, that is, we can find a quasiconformal map $\varphi$ of a neighbourhood $W$ of the filled Julia set $K_{f}$ in $U$ into $\mathbb{C}$ which satisfies

i) $\varphi\left(K_{f}\right)=K_{P}$,

ii) $\mu(\varphi)=0$ almost everywhere on $K_{f}$,

iii) $\varphi \circ f=P \circ \varphi$ on $W \cap f^{-1}(W)$.

Moreover, if $K_{f}$ is connected, then $P$ is unique up to conjugation by an affine map.

A corollary of this is that for every parameter $c$ in the main cardioid of the Mandelbrot set, the Julia set $\mathcal{J}\left(P_{c}\right)$ is a quasicircle, that is, the image of a circle under a quasiconformal map.

\subsection{Hausdorff and kernel convergence}

The starting point of the present work is a family of functions $g_{\lambda}(z)=z-1+\lambda z e^{z}$ having an invariant Baker domain for every parameter $\lambda$. Then we perturb this family in a way that the Baker domain vanishes and in its place an attracting basin "appears". The perturbed functions are $g_{\lambda, \mu}(z)=(1-\mu)\left(z-1+\lambda z e^{z}\right)$ and we obtain that $g_{\lambda, \mu} \rightarrow g_{\lambda}$ uniformly on compact subsets of $\mathbb{C}$ as $\mu \rightarrow 0$.

In order to prove the convergence also in a dynamical sense, we use the notion of Hausdorff convergence for compact sets and the kernel convergence for open sets, 
presented in this section. We begin with the notion of kernel given by Golusin in the 1950's; see 31.

Definition 1.1. (Kernel) Let $I$ be an index set and $\left\{B_{j}\right\}_{j \in I}$ be a sequence of connected domains in $\mathbb{C}$ all of them containing a marked point $z_{0} . A$ domain $B$ is called a kernel of the sequence $\left\{B_{j}\right\}_{j \in I}$ if it is the largest connected domain containing the marked point $z_{0}$ and satisfying the property that any compact subset $K$ of $B$ is contained in all $B_{j}$ except for at most finitely many of them.

We may compare this definition with the ones used by Krauskopf and Kriete in [42, and by Morosowa in [53], who omitted the concept of marked point. Instead of this, Morosawa required that if an open set $U$ is contained in $\left\{B_{j}\right\}$ for infinitely many $j$, then $U$ should be contained as well in $B$. In contrast to this, Krauskopf and Kriete called $B$ a kernel if it is the maximal open set satisfying the compact set property, that is, if this property is not held by any domain $\tilde{B}$ with $B \subsetneq \tilde{B}$. Hence in both cases, the requirement that the marked point $z_{0}$ is contained in all $\left\{B_{j}\right\}$ and therefore also in $B$ may be replaced by a neighbourhood of $z_{0}$.

Even though these three definitions are equivalent, Golusin's definition using a marked point is more detailed. Notice that the marked point $z_{0}$ is useful for the uniqueness of the kernel with respect to $z_{0}$. Otherwise, the kernel may not be unique in the sense that there may be more than one kernel for a given sequence of domains each one corresponding to a different marked point. In this case, Krauskopf and Kriete admitted a kernel as the union of two or more simply connected domains. Now we define the Kernel Convergence in the sense of Carathéodory; see [31].

Definition 1.2. Let $I$ be an index set. We say that a sequence of connected domains $\left\{B_{j}\right\}_{j \in I}$ converges to the kernel $B$ if the domain $B$ is a kernel of every subsequence of $\left\{B_{j}\right\}_{j \in I}$.

If a sequence of domains converges to a domain $B$ in the sense that every boundary point of $B_{j}$ is arbitrarily closed to the boundary points of $B$ for infinitely many $j$ 's, then the domain $B$ is a kernel of the sequence $\left\{B_{j}\right\}$ and the convergence as kernel is assured. In particular, if the sequence of domains $\left\{B_{j}\right\}$ satisfies $B_{1} \supset B_{2} \supset B_{3} \cdots$ or $B_{1} \subset B_{2} \subset B_{3} \cdots$, then $\left\{B_{j}\right\}$ converges to its kernel.

Now consider the Riemann sphere $\widehat{\mathbb{C}}$ with the chordal metric. The Hausdorff distance between two non-empty compact sets $A, B \subset \widehat{\mathbb{C}}$ is defined in the usual way as

$$
\operatorname{dist}_{H}(A, B):=\inf \left\{\varepsilon>0 \mid A \in U_{\varepsilon}(B) \text { and } B \in U_{\varepsilon}(A)\right\}
$$

where $U_{\varepsilon}(X)$ denotes the $\varepsilon$-neighbourhood of a compact set $X \subset \widehat{\mathbb{C}}$. For sequences of index sets $\left\{I_{n}\right\}_{n \in \mathbb{N}}$ we define the convergence in the Hausdorff metric as follows. 
Definition 1.3. Let $K$ and $\left\{K_{j}\right\}_{j \in I_{n}}$ be non-empty compact sets in $\widehat{\mathbb{C}}$. Then $K_{j}$ converges to $K$ in the Hausdorff metric, if $\operatorname{dist}_{H}\left(K, K_{j}\right) \rightarrow 0$ as $n \rightarrow \infty$.

In the Riemann sphere or in the complex plane we have the following relation between Hausdorff and kernel convergence.

Lemma 1.20. Let $K_{j}$, with $j \in I_{n}$, and $K$ be nonempty compact subsets of $\widehat{\mathbb{C}}$. Then $\operatorname{dist}_{H}\left(K_{j}, K\right) \rightarrow 0$ as $n \rightarrow \infty$ if and only if the following two conditions hold:

- Each component $B$ of $K^{c}:=\widehat{\mathbb{C}} \backslash K$ is a kernel of a sequence of components $B_{j}$ of $K_{j}^{c}:=\widehat{\mathbb{C}} \backslash K_{j}$, and

- Every kernel of an infinite subsequence $B_{j_{n}}$ of components of $K_{j_{n}}^{c}$ is a component of $K^{c}$.

One direction of the Hausdorff convergence of Julia sets is satisfied almost immediately by the uniform convergence of the functions. This fact is true since the Julia set is the closure of the repelling fixed points. The latter will be close to each other using the uniform convergence and Rouché's Theorem. This is also known as lower semi-continuity of Julia sets; see [22] for a proof due to Douady.

Lemma 1.21. If $f_{n}$ converges to $f$ locally uniformly on compact subsets, then for an arbitrary $\varepsilon>0$ there exists an $N \in \mathbb{N}$ such that

$$
\mathcal{J}(f) \subset U_{\varepsilon}\left(\mathcal{J}\left(f_{n}\right)\right)
$$

for all $n>N$. 


\subsection{Notation}

Here we present a list of notations used in the present work, for clarification.

$\begin{array}{ll}\mathbb{N} & \{0,1,2, \ldots\} . \\ \mathbb{C} & \text { Complex plane. } \\ \widehat{\mathbb{C}}=\mathbb{C} \cup \infty & \text { Riemann sphere. } \\ \mathbb{D}=\{z \in \mathbb{C}:|z|<1\} & \text { Unit disc. } \\ D(x, \varepsilon) & \text { Disc of radius } \varepsilon \text { centered at the point } x . \\ d(a, b) & \text { Euclidian distance between } a \text { and } b . \\ \operatorname{dist}(x, A) & \text { Distance from a point } x \text { to a set } A \text { defined as } \\ & \text { inf }\{d(x, a): a \in A\} \text {. } \\ \operatorname{dist}_{H}(A, B) & \text { Hausdorf distance between two non empty } \\ A \subset \subset B & \text { compact sets } A \text { and } B \text {. } \\ a \approx b & A \text { is relatively compact in } B . \\ f(z) \simeq g(z) & b \text { is a numerical approximation of } a . \\ & g(z) \text { is the linear approximation of } f(z) \\ a \approx b & \text { by means of its Taylor expansion. } \\ P(f) & b \text { is an analytical approximation of } a . \\ \mathcal{F} & \text { forward orbit of the set of singularities of } f . \\ \mathcal{J} & \text { Fatou set. } \\ \mathcal{K} & \text { Julia set. } \\ O^{+}(z), O^{-}(z) & \text { Filled Julia set. } \\ \mathcal{O}(x) & \text { Forward and backward orbits of } z . \\ & \text { Big } \mathcal{O} \text { of x. }\end{array}$




\section{Dynamical description of the family $g_{\lambda, \mu}$}

In this chapter we present a study of the dynamical properties of the family of functions $g_{\lambda, \mu}(z)=(1-\mu)\left(z-1+\lambda z e^{z}\right)$ as the real parameter $\mu<\frac{1}{2}$ tends to zero and where $\lambda$ is a non-zero complex constant. It is easy to see that $g_{\lambda, \mu}$ converges uniformly to $g_{\lambda}(z)=z-1+\lambda z e^{z}$ on compact subsets of $\mathbb{C}$. In Chapter 3 we prove results about the convergence of $g_{\lambda, \mu}$ to $g_{\lambda}$ in a dynamical sense, as the Hausdorff convergence of the Julia sets and the kernel convergence of the different Fatou components. Keeping this in mind here we present some properties of the Fatou set $\mathcal{F}\left(g_{\lambda, \mu}\right)$.

The Fatou set of $g_{\lambda, \mu}$ has an attracting basin $\mathcal{A}_{\mu}$ containing the fixed point $z_{\mu}$, which in turn is contained in a neighbourhood of $1-\frac{1}{\mu}$. In [48], Lauber showed that the limit function $g_{\lambda}$ has an unbounded absorbing domain $\mathcal{M}_{\delta}$ contained in a left half-plane. Iterates of points $z$ in $\mathcal{M}_{\delta}$ with large negative real part behave like iterates under the mapping $z \mapsto z-1$, and so he deduced that $\mathcal{M}_{\delta}$ is contained in a Baker domain $\mathcal{B}_{\lambda}$. In the approximating process by the function $g_{\lambda, \mu}$ as $\mu \rightarrow 0$, the fixed point $z_{\mu}$ of $g_{\lambda, \mu}$ tends to $-\infty$. In the limit, the attracting basin of $\mathcal{A}_{\mu}$ becomes the Baker domain $\mathcal{B}_{\lambda}$.

In particular, it was also proved by Lauber that $\mathcal{B}_{\lambda}$ contains all critical points of $g_{\lambda}$ except at most one, which we denote by $c_{0}$. That is, for certain values of $\lambda$ there is at most one free critical point, and in case that $c_{0} \notin \mathcal{B}_{\lambda}$ then $\mathcal{F}\left(g_{\lambda}\right)$ has one non-repelling cycle. We prove that this behaviour is also exhibited by $g_{\lambda, \mu}$, independent of the value of $\mu$. In Section 2.2 we analyse as an explicit example the 
behaviour of the critical point $c_{0}$ for $\lambda \in \mathbb{R}$ and $g_{\lambda, \mu}$ restricted to the real line. For positive values of $\lambda$ the critical point $c_{0}$ belongs always to $\mathcal{A}_{\mu}$. Then there exists a value $q \in(-2,0)$ such that depending on the value of $\lambda$ one of the following possibilities holds

- $c_{0}$ lies in $\mathcal{A}_{\mu}$ for $q<\lambda<0$,

- $c_{0}$ lies in a basin of attraction denoted by $\mathcal{A}_{0}$ for $r<\lambda<q$,

- $c_{0}$ lies in $\mathcal{J}\left(g_{\lambda, \mu}\right)$ for $\lambda \leq r$,

whereby $r \approx-5$ and $\mathcal{A}_{0} \cap \mathcal{A}_{\mu}=\emptyset$. Note that the critical point $c_{0}$ is not real anymore when $\lambda \notin \mathbb{R}$. Using continuity arguments, this behaviour is satisfied by parameters $\lambda$ in a domain $\mathcal{H}_{1}$ in $\mathbb{C}$ such that $\mathcal{H}_{1} \cap \mathbb{R}=(r, q)$. In other words, for complex parameters $\lambda \in \mathcal{H}_{1}$ the function $g_{\lambda, \mu}$ has an attracting fixed point different from $z_{\mu}$. In the following chapter in Section 3.2 we show the existence of attracting fixed points of $g_{\lambda, \mu}$ which converge to parabolic fixed points of $g_{\lambda}$.

In order to give a first geometrical approach of the basin of attraction $\mathcal{A}_{\mu}$ we construct an invariant absorbing domain $\mathcal{H}_{\eta, \rho} \subset \mathcal{A}_{\mu}$ and prove that a finite number of critical points (and hence critical values) are contained in $\mathcal{H}_{\eta, \rho}$. Since the behaviour under iterates of $g_{\lambda, \mu}$ is unknown for critical points with large imaginary part, we cannot exclude the existence of further Fatou components distinct from the attracting basin $\mathcal{A}_{\mu}$ and the non-repelling cycle attached to the free critical point $c_{0}$.

We construct for every arbitrary but fixed parameter $\lambda$ a family of Jordan curves $\left\{\Gamma_{k, \mu}\right\}_{k \in \mathbb{Z}^{*}}$ depending on $\mu \in\left(0, \frac{1}{2}\right)$ where $\Gamma_{k, \mu}: t \mapsto \gamma_{k, \mu}(t)$ for $t \geq 0$. These curves improve the understanding of the dynamics of the family $g_{\lambda, \mu}$. For each $k$, with $|k| \leq N, \Gamma_{k, \mu}$ contains a simple curve segment joining the critical point $c_{k}$ to the fixed point $z_{\mu}$. Since we construct a Jordan curve for each $c_{k}$ contained in $\mathcal{A}_{\mu}$, we obtain a finite number of curves $\Gamma_{k, \mu}$. The definition $\gamma_{k, \mu}(0):=z_{\mu}$ is given in a natural way by construction. Furthermore, the curves $\Gamma_{k, \mu} \backslash\left\{z_{\mu}\right\}$ are pairwise disjoint and as $t \rightarrow \infty$ we have that $\operatorname{Re} \gamma_{k, \mu}(t) \rightarrow \infty$ while $\operatorname{Im} \gamma_{k, \mu}(t)$ is bounded. For each $k$ and $\mu$, the curve $\Gamma_{k, \mu} \cup\left(-\infty, z_{\mu}\right)$ is completely invariant. The latter implies that $\mathcal{A}_{\mu}$ contains a set of disjoint horizontal right strips, that is, a set of strips bounded to the left and unbounded to the right which are asymptotically horizontal for points with large positive real part.

The structure gave by the Jordan curves enable us to delimit a domain on which we function $g_{\lambda, \mu}$ "behaves like" a polynomial. This kind of "behaviour" is better known as polynomial-like mapping. We show that for any given $d \in \mathbb{N}$, with $d \geq 2$, there exists a bounded domain $V$ where $\left.g_{\lambda, \mu}\right|_{V}$ is a proper map of degree 
at most $2 d$. Therefore, the triple $\left(g_{\lambda, \mu}, V, g_{\lambda, \mu}(V)\right)$ is polynomial-like as defined in the introduction in Section 1.8 ,

In Section 2.1 we prove the existence of the attracting basin $\mathcal{A}_{\mu}$. In Section 2.2 we present the analysis of $g_{\lambda, \mu}$ restricted to the real line followed by some properties for the critical points and values in Section 2.3. The sequent Section 2.4 is divided into two subsections, devoted to the construction of the absorbing domain $\mathcal{H}_{\eta, \rho}$ and to the proof of its invariance under $g_{\lambda, \mu}$ respectively. Finally we show in Section 2.5 the construction of the family of Jordan curves $\Gamma_{k, \mu}$ followed by the prove of the polynomial-like mapping's property in Section 2.6. During the present chapter we mostly omit in the notation the parameter $\lambda$ for simplicity, as for example in $\mathcal{A}_{\mu}$ instead of $\mathcal{A}_{\mu, \lambda}$, even though the results depend on the parameter $\lambda$.

\subsection{Basin of attraction $\mathcal{A}_{\mu}$}

We begin the description of the dynamical properties of the function

$$
g_{\lambda, \mu}(z)=(1-\mu)\left(z-1+\lambda z e^{z}\right), \quad \text { with } \quad \mu \in\left(0, \frac{1}{2}\right) .
$$

by showing that it has a basin of attraction $\mathcal{A}_{\mu}$ for every $\lambda \in \mathbb{C}^{*}$ and for sufficiently small $\mu_{0}$, with $\mu_{0}=\mu_{0}(\lambda)$. We firstly prove that $g_{\lambda, \mu}$ has an attracting fixed point for every $\mu \leq \mu_{0}$.

Theorem 2.1. Let $\lambda \in \mathbb{C}^{*}$ be any arbitrary but fixed parameter. Then for any $\varepsilon \in(0,1]$ there exists a $\mu_{1} \in\left(0, \frac{1}{2}\right)$ sufficiently small that if $|\lambda|<\frac{\mu^{2} \varepsilon \exp (1 / \mu-1-\varepsilon)}{|\mu-\varepsilon \mu-1|(1-\mu)}$ for all $\mu \leq \mu_{1}$, then the function $g_{\lambda, \mu}$ has a unique fixed point $z_{\mu}$ in $D\left(1-\frac{1}{\mu}, \varepsilon\right)$ which is always attracting.

Proof. During the present proof, let $p_{\mu}(z)=(1-\mu)(z-1)$ be a family of polynomials depending on $\mu$. These functions have a unique fixed point at $1-\frac{1}{\mu}$. Using Rouché's theorem we claim that $g_{\lambda, \mu}(z)-z$ and $p_{\mu}(z)-z$ have the same number of zeros in $D\left(1-\frac{1}{\mu}, \varepsilon\right)$ if $\left|\left(g_{\lambda, \mu}(z)-z\right)-\left(p_{\mu}(z)-z\right)\right|<\left|p_{\mu}(z)-z\right|$ for $z \in \partial D\left(1-\frac{1}{\mu}, \varepsilon\right)$. For such points $z$, we have $\left|z-1+\frac{1}{\mu}\right|=\varepsilon$. Then they satisfy $|z| \leq\left|1-\frac{1}{\mu}-\varepsilon\right|$ and $\operatorname{Re} z \leq 1-\frac{1}{\mu}+\varepsilon<0$ for all $\mu<\frac{1}{2}$. Let $\mu_{1}=\mu_{1}(\lambda)$ be sufficiently small that the inequality

$$
|\lambda|<e^{\frac{1}{\mu}-1-\varepsilon} \frac{\varepsilon \mu^{2}}{|\mu-\varepsilon \mu-1|(1-\mu)}
$$


is satisfied for all $\mu \leq \mu_{1}$. This is equivalent to

$$
\begin{aligned}
& & |\lambda|\left|1-\frac{1}{\mu}-\varepsilon\right| e^{1-\frac{1}{\mu}+\varepsilon} & <\frac{\varepsilon \mu}{(1-\mu)} \\
& \Longleftrightarrow \quad & |\lambda||z| e^{\operatorname{Re} z} & <\frac{\varepsilon \mu}{(1-\mu)} \\
& \Longleftrightarrow \quad & \left|(1-\mu)\left(\lambda z e^{z}\right)\right| & <\varepsilon \mu=|\mu|\left|z-1+\frac{1}{\mu}\right| .
\end{aligned}
$$

Since $\left|g_{\lambda, \mu}(z)-p_{\mu}(z)\right|=\left|(1-\mu)\left(\lambda z e^{z}\right)\right|$ equals the left side from the inequality above and $\left|p_{\mu}(z)-z\right|=|-\mu z-(1-\mu)|$ the right side, we can conclude by Rouché's theorem that $g_{\lambda, \mu}$ has a unique fixed point as desired, which we denote by $z_{\mu}$.

We now show that for points $z \in D\left(1-\frac{1}{\mu}, \varepsilon\right)$ the multiplier $g_{\lambda, \mu}^{\prime}$ has modulus smaller than one. For such points $z$, the point $z+1$ lies in the disc $D\left(2-\frac{1}{\mu}, \varepsilon\right)$ implying that $|z+1|<\left|2-\frac{1}{\mu}-\varepsilon\right|$ and $\operatorname{Re} z<1-\frac{1}{\mu}+\varepsilon$. Let $\mu_{2}=\mu_{2}(\lambda)$ be sufficiently small that the inequility

$$
|\lambda|<e^{\frac{1}{\mu}-1-\varepsilon} \frac{\mu^{2}}{(1-\mu)(1-2 \mu+\varepsilon \mu)}
$$

holds for every $\mu \leq \mu_{2}$. Equivalently we obtain

$$
\begin{array}{rlrl} 
& & |\lambda| e^{1-\frac{1}{\mu}+\varepsilon}\left(\frac{1}{\mu}-2+\varepsilon\right) & <\frac{\mu}{1-\mu} \\
& \Longrightarrow & (1-\mu)|\lambda| e^{\operatorname{Re} z}|z+1| & <\mu \\
\Longleftrightarrow & & (1-\mu)+(1-\mu)\left|\lambda e^{z}(z+1)\right| & <1 \\
\Longrightarrow & (1-\mu)\left|1+\lambda e^{z}(z+1)\right| & <1 .
\end{array}
$$

We finally show that given a $\mu$ satisfying condition $(2.2)$, then Equation $(2.3)$ is also satisfied for this $\mu$. In other words, if $g_{\lambda, \mu}$ has a fixed point $z_{\mu}$ in the disc $D\left(1-\frac{1}{\mu}, \varepsilon\right)$, then it is attracting. Comparing the above conditions for $\lambda$ we have

$$
\begin{aligned}
e^{\frac{1}{\mu}-1-\varepsilon} \frac{\varepsilon \mu^{2}}{|\mu-\varepsilon \mu-1|(1-\mu)} & <e^{\frac{1}{\mu}-1-\varepsilon} \frac{\mu^{2}}{(1-\mu)(1-2 \mu+\varepsilon \mu)} \\
\varepsilon<\frac{|\mu-\varepsilon \mu-1|}{1-2 \mu+\varepsilon \mu} & =\frac{1-\mu+\varepsilon \mu}{1-2 \mu+\varepsilon \mu},
\end{aligned}
$$

which is true for all $0<\varepsilon \leq 1$ and $\mu<1$. Hence for any given $\lambda$ the maximum value $\mu_{1}$ suffices and the fixed point $z_{\mu}$ is attracting for all $\mu \leq \mu_{1}$. 
Corollary 2.2. For every $\lambda \in \mathbb{C}^{*}$ there exists $\mu_{0}=\mu_{0}(\lambda) \in\left(0, \frac{1}{2}\right)$ such that for every $\mu \leq \mu_{0}$ satisfying

$$
|\lambda|<e^{\frac{1}{\mu}-2} \frac{\mu^{2}}{1-\mu} .
$$

$g_{\lambda, \mu}$ has a unique attracting fixed point $z_{\mu}$ in a neighbourhood of radius 1 around $1-\frac{1}{\mu}$.

The corollary follows directly from the theorem above by taking the value $\varepsilon=1$, for which the desired result is satisfied and simplifies the notation. We remark at this point that the function $g_{\lambda, \mu}$ may have another attracting fixed point outside the disc $D\left(1-\frac{1}{\mu}, 1\right)$ for some values of $\lambda \in \mathbb{C}^{*}$. In particular we prove the latter statement in Subsection 2.2 .2 in Theorem 2.8 for some real parameters $\lambda<0$.

\subsection{Dynamics on the real line}

Here we describe briefly the dynamics of $g_{\lambda, \mu}$ considered as a real function. It is easy to see that if $\lambda$ is in $\mathbb{R}^{*}=\mathbb{R} \backslash 0$, then $g_{\lambda, \mu}(\mathbb{R}) \subset \mathbb{R}$. Hence it is reasonable to analyse $g_{\lambda, \mu}(x)$ for $x \in \mathbb{R}$ and $\lambda \in \mathbb{R}^{*}$. We show that there exist either one or two attracting fixed points depending on the value of $\lambda$. The first one, $x_{\mu}$, corresponds to the fixed point $z_{\mu}$ presented in the previous section and exists for every $\lambda$. For certain values of $\lambda<0$ we prove the existence of an additional attracting fixed point $x_{r}$ close to the origin by analysing the behaviour of the critical point $c_{0}$.

In this section we write $g_{\lambda, \mu}(x)$ and its derivatives as follows

$$
\begin{aligned}
& g_{\lambda, \mu}(x)=(1-\mu)\left(x-1+\lambda x e^{x}\right), \\
& g_{\lambda, \mu}^{\prime}(x)=(1-\mu)\left(1+\lambda e^{x}+\lambda x e^{x}\right), \\
& g_{\lambda, \mu}^{\prime \prime}(x)=(1-\mu)\left(\lambda e^{x}\right)(x+2) .
\end{aligned}
$$

We can easily see that $g_{\lambda, \mu}^{\prime \prime}(x)=0$ if and only if $x=-2$. Therefore $g_{\lambda, \mu}^{\prime}(x)$ has a change of slope only at the point $x=-2$ and the value $g_{\lambda, \mu}^{\prime}(-2)$ will determine the number of critical points of $g_{\lambda, \mu}$.

Before pursing this line of argument, we firstly present an alternative proof for the existence of $x_{\mu}$ restricted to the real line. This alternative proof gives some insight which is helpful for the proof of the existence of further fixed points.

Theorem 2.3. For every $\lambda \in \mathbb{R}^{*}$, there exists $\delta \in(0,1]$ and $\mu_{1} \in(0,1 / 3)$ such that $g_{\lambda, \mu}(x)$ has an attracting fixed point $x_{\mu} \in\left(1-\frac{1}{\mu}-\delta, 1-\frac{1}{\mu}+\delta\right)$ for all $\mu \leq \mu_{1}$. 
Proof. At any fixed point, we have that $g_{\lambda, \mu}(x)$ has a fixed point if and only if $(1-\mu)\left(\lambda x e^{x}\right)=x-(1-\mu)(x-1)$, which is in turn equivalent to

$$
\begin{aligned}
\lambda x e^{x} & =\frac{x \mu}{1-\mu}+1 \quad \text { or to } \\
\lambda e^{x} & =\frac{\mu}{1-\mu}+\frac{1}{x} .
\end{aligned}
$$

Write $h(x)=\frac{\mu}{1-\mu}+\frac{1}{x}$ for the function on the right side in the latter equation. On the one hand, we have that $h(x)$ is strictly monotonic decreasing for every $x$ and has negative values for $x$ in $\left(1-\frac{1}{\mu}, 0\right)$. In other words, $h(x)=0$ only for $x=1-\frac{1}{\mu}$. Then for every $\varepsilon>0$, there exists a $\delta$ (w.l.o.g. $\delta \leq 1$ ) such that if $x$ lies in the interval $\left(1-\frac{1}{\mu}-\delta, 1-\frac{1}{\mu}+\delta\right)$, then $|h(x)|<\varepsilon$.

On the other hand, we have that $\lambda e^{x}$ is strictly monotonic increasing (resp. decreasing) for $\lambda>0$ (resp. for $\lambda<0$ ) and with values greater (resp. smaller) than zero. We also know that $\lambda e^{x} \rightarrow 0$ as $x \rightarrow-\infty$. Thus, for any $\lambda$ and any given $\varepsilon$ there exists $\mu_{1}<\frac{1}{3}$ sufficiently small that $\left|\lambda e^{1-\frac{1}{\mu}}\right|<\varepsilon$ for every $\mu \leq \mu_{1}$. Finally, there exists an $x_{\mu} \in\left(1-\frac{1}{\mu}-\delta, 1-\frac{1}{\mu}+\delta\right)$ such that $\lambda e^{x_{\mu}}=h\left(x_{\mu}\right)$. If $\lambda>0$, then $\lambda e^{x}>0$ and with this $x_{\mu}<1-\frac{1}{\mu}$, resp. $x_{\mu}>1-\frac{1}{\mu}$ for $\lambda<0$.

To show that the fixed point $x_{\mu}$ is attracting, we prove the inequality $\left|g_{\lambda, \mu}^{\prime}(x)\right|<1$ for points in the interval $\left[-\frac{1}{\mu}, 2-\frac{1}{\mu}\right]$ which contains $\left(1-\frac{1}{\mu}-\delta, 1-\frac{1}{\mu}+\delta\right)$ for $\delta \leq 1$. If $\mu<\frac{1}{4}$, then $2-\frac{1}{\mu}<-2$ holds and it suffice to prove the inequality for the boundary points, since $g_{\lambda, \mu}^{\prime}$ is either increasing for $\lambda>0$ or decreasing for $\lambda<0$. For $1 / 4 \leq \mu<1 / 3$, we obtain $-2 \leq 2-\frac{1}{\mu}<-1$ and the global minimum (resp. maximum) of $g_{\lambda, \mu}^{\prime}$ is inside the interval. Therefore, we compute the multiplier at the boundary values $\left\{-\frac{1}{\mu}, 2-\frac{1}{\mu}\right\}$ and at the extreme point -2 .

Let $x_{0}$ be an arbitrary fixed point. We use equality (2.6) to simplify the derivative as follows

$$
g_{\lambda, \mu}^{\prime}\left(x_{0}\right)=(1-\mu)\left(2+\frac{1}{x_{0}}+\frac{\left(x_{0}+1\right) \mu}{1-\mu}\right) .
$$

After simple computations and using the equation above we obtain for $x_{0}=-\frac{1}{\mu}$ that $\left|g_{\lambda, \mu}^{\prime}\left(-\frac{1}{\mu}\right)\right|=(1-\mu)^{2}<1$ for all $\mu<1$. In the same way, we compute for $x_{0}=2-\frac{1}{\mu}$ the multiplier $\left|g_{\lambda, \mu}^{\prime}\left(2-\frac{1}{\mu}\right)\right|=(1-\mu)\left|1-\frac{\mu(3 \mu-1)}{(1-\mu)(2 \mu-1)}\right|$. For $\mu<1 / 3$ 
the inequality $0<\frac{\mu(3 \mu-1)}{(1-\mu)(2 \mu-1)}<1$ holds if and only if $\mu(3 \mu-1)>(1-\mu)(2 \mu-1)$ which is in turn equivalent to $5 \mu^{2}-4 \mu+1>0$. We simplify the equation as $5 \mu^{2}-4 \mu+1=5\left(\left(\mu-\frac{2}{5}\right)^{2}+\frac{1}{25}\right)$, showing that the multiplier has norm smaller than one.

Finally, using Equation (2.7) we compute the multiplier at the extreme point $x=-2$ as

$$
\left|g_{\lambda, \mu}^{\prime}(-2)\right|=(1-\mu)\left|\frac{3}{2}-\frac{\mu}{1-\mu}\right|=\left|\frac{3-5 \mu}{2}\right|
$$

which is smaller than one for $\frac{1}{5}<\mu<1$, including the interval $\left[\frac{1}{4}, \frac{1}{3}\right]$. With this we conclude that the multiplier for any fixed point in $\left[-\frac{1}{\mu}, 2-\frac{1}{\mu}\right]$ and every $0<\mu \leq \mu_{1}<1 / 3$ is smaller than one.

Among the properties of $g_{\lambda, \mu}$ as a real function it is important to remark that $g_{\lambda, \mu}(0)=-1+\mu \in(-1,0)$ for all $\lambda$ and $0<\mu<1$, that is, $x=0$ is never a fixed point. Furthermore,

$$
g_{\lambda, \mu}^{\prime}(0)=(1-\mu)(1+\lambda)
$$

which is greater or smaller than zero depending on $\lambda$. As we mentioned before, the value $g_{\lambda, \mu}^{\prime}(-2)=(1-\mu)\left(1-\lambda e^{-2}\right)$ also directly depends on the sign of $\lambda$. Therefore we divide the analysis into two subsections considering $\lambda>0$ and $\lambda<0$ separately. Furthermore, we search for the number of fixed points pursing the analysis for $x<0$ and $x>0$ also independently, since 0 is never a fixed point. We remark here that

$$
g_{\lambda, \mu}^{\prime}(-1)=1-\mu>0 \quad \text { for all } \lambda .
$$

With this, the value $g_{\lambda, \mu}(-1)$ will be useful for locating the critical points of $g_{\lambda, \mu}$ on $\mathbb{R}$.

\subsubsection{Dynamics for $\lambda>0$}

During this subsection, let $\lambda>0$ and $\mu<1 / 3$. We show that for these values of $\lambda$ there exist exactly one attracting fixed point and one repelling fixed point, and the number of critical points changes as $\lambda$ varies.

Theorem 2.4. For $\lambda>0, g_{\lambda, \mu}(x)$ has exactly two fixed points. The first is the attracting fixed point $x_{\mu}$, and the second is the fixed point $x_{r}$, which is always repelling.

Proof. We follow the line of argument in the proof of Theorem 2.3. Using Equation (2.6), we obtain that $h(x)=\frac{\mu}{1-\mu}+\frac{1}{x}>0$ if and only if $x<\frac{\mu-1}{\mu}$ or $x>0$. 
Furthermore $h^{\prime}(x)=-\frac{1}{x^{2}}$ is negative for every $x$. Since $\lambda e^{x}$ and its derivative are always positive for $\lambda>0$, there are exactly two fixed points of $g_{\lambda, \mu}(x)$, that is, there exist two $x$ values where $\lambda e^{x}=h(x)$. The first one is $x_{\mu}$ and lies in the interval $\left(1-\frac{1}{\mu}-\delta, 1-\frac{1}{\mu}\right)=\left(-\frac{1}{\mu}, 1-\frac{1}{\mu}\right)$ for $\delta=1$. It was proved in Theorem 2.3 that it is attracting. The second fixed point, denoted by $x_{r}$, is contained in the interval $(0, \infty)$.

We show now that $x_{r}$ is always repelling. Using Equation (2.7) for the derivative of fixed points, we obtain

$$
\begin{aligned}
g_{\lambda, \mu}^{\prime}\left(x_{r}\right) & =(1-\mu)\left(2+\frac{1}{x_{r}}\right)+\mu\left(x_{r}+1\right) \\
& >\left(\frac{1}{2}\right)\left(2+\frac{1}{x_{r}}\right)+\mu\left(x_{r}+1\right) \quad \text { for } \mu<\frac{1}{2} \\
& =1+\frac{1}{2 x_{r}}+\mu\left(x_{r}+1\right)>1 \quad \text { for every } x_{r}>0,
\end{aligned}
$$

yielding the result.

We continue our analysis by giving the number of critical points.

Lemma 2.5. For $\lambda>0, g_{\lambda, \mu}(x)$ has either none, one or two real critical points. Furthermore, the global minimum of $g_{\lambda, \mu}^{\prime}(x)$ is at $x=-2$.

Proof. After computing the second derivative in 2.5$)$, we see that $g_{\lambda, \mu}^{\prime \prime}(x) \leq 0$ for every $x \leq-2$. Hence $g_{\lambda, \mu}^{\prime}(x)=(1-\mu)\left(1+\lambda(x+1) e^{x}\right)$ has its global minimum at $x=-2$ for every $\lambda>0$. Furthermore, as $x \rightarrow-\infty$ we obtain that $\lambda(x+1) e^{x}$ is negative and tends to zero. Then $g_{\lambda, \mu}^{\prime}(x)$ tends to $1-\mu$ from below as $x \rightarrow-\infty$. Hence there exists a negative $s$ sufficiently large that $g_{\lambda, \mu}^{\prime}(x)>0$ for all $x \leq s$. Recall that $g_{\lambda, \mu}^{\prime}(-1)=1-\mu$ is always positive and $g_{\lambda, \mu}^{\prime}(x) \rightarrow \infty$ as $x \rightarrow \infty$.

Now consider $g_{\lambda, \mu}^{\prime}(-2)=(1-\mu)\left(1-\lambda e^{-2}\right)$ as a function of $\lambda$. It follows that the value $g_{\lambda, \mu}^{\prime}(-2)$ lies on a line with negative slope $-(1-\mu) e^{-2}$ and equals zero at $\lambda=e^{2}$. In other words, the $g_{\lambda, \mu}^{\prime}(-2)$ may be positive, equal zero or negative, depending on the parameter $\lambda$, yielding the result.

As a direct conclusion from the previous lemma we obtain the following statements:

i) For $0<\lambda<e^{2}$, we have $g_{\lambda, \mu}^{\prime}(-2)>0$ and with this $g_{\lambda, \mu}^{\prime}$ has no real zeros. 


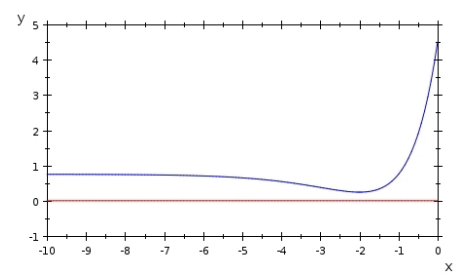

a) $\lambda=5$

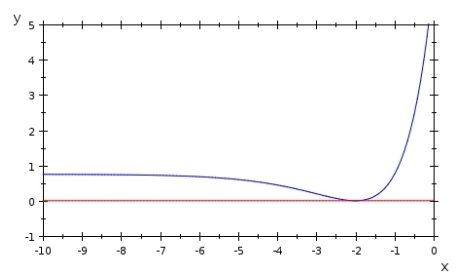

b) $\lambda=e^{2}$

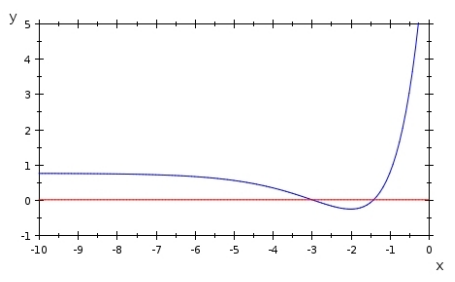

c) $\lambda=10$

Figure 2.1: Graphs of the derivative $g_{\lambda, \mu}^{\prime}(x)=(1-\mu)\left(1+\lambda e^{x}+\lambda x e^{x}\right)$ for $\mu=\frac{1}{4}$ and some positive values of $\lambda$. As $\lambda$ increases from zero, the function $g_{\lambda, \mu}$ has no real critical points, a unique critical point $c_{0}=-2$, or two different critical points, denoted by $c_{01}$ and $c_{02}$.

ii) For $\lambda=e^{2}$, the point $x=-2$ is a zero of both $g_{\lambda, \mu}^{\prime}$ and $g_{\lambda, \mu}^{\prime \prime}$. Hence $g_{\lambda, \mu}$ has a unique critical point $c_{0}$. This is the only parameter for which a critical point is not simple.

iii) For $\lambda>e^{2}, g_{\lambda, \mu}^{\prime}(-2)<0$ and since $g_{\lambda, \mu}^{\prime}(-1)>0$ (see Equation 2.9), we conclude that $g_{\lambda, \mu}^{\prime}(x)$ has two zeros aligned as $c_{02}<-2<c_{01}<-1$.

iv) The critical point $c_{02}$ is in the interval $\left(2-\frac{1}{\mu},-2\right)$ as we prove below in Lemma 2.6.

The position of the critical point $c_{02}$ has a direct connection with the basin of attraction of $x_{\mu}$. Therefore we recall the maximum value of $\mu_{1}=\mu_{1}(\lambda)$ as stated in the Inequality (2.4) such that the attracting fixed point exists for every $\mu \leq \mu_{1}$. Lemma 2.6. Let $\mu \leq \mu_{2}=\min \left\{\mu_{1}, 1 / 3\right\}$, where $\mu_{1}$ is such that $|\lambda|<e^{\frac{1}{\mu}-2} \frac{\mu^{2}}{1-\mu}$ for all $\mu<\mu_{1}$. Then the critical point $c_{02}$ lies in the interval $\left(2-\frac{1}{\mu},-2\right)$.

Proof. For $\lambda<e^{2}$ the functions $g_{\lambda, \mu}$ has no critical points. Therefore we consider only $\lambda>e^{2}$. In this case, we proved in Lemma 2.5 that $g_{\lambda, \mu}^{\prime}(-2)<0$ and it suffices to show that $g_{\lambda, \mu}^{\prime}\left(2-\frac{1}{\mu}\right)>0$. After some computations we have that $g_{\lambda, \mu}^{\prime}\left(2-\frac{1}{\mu}\right)=(1-\mu)\left(1+\lambda e^{2-\frac{1}{\mu}}\left(3-\frac{1}{\mu}\right)\right)$ is positive if and only if

$$
\lambda e^{2-\frac{1}{\mu}}<\frac{-\mu}{3 \mu-1}
$$


From the hypothesis on $\mu$ and $\lambda$, we have on the one hand

$$
\lambda e^{2-\frac{1}{\mu}}<e^{\frac{1}{\mu}-2}\left(\frac{\mu^{2}}{1-\mu}\right) e^{2-\frac{1}{\mu}}=\frac{\mu^{2}}{1-\mu},
$$

and on the other hand

$$
\frac{\mu^{2}}{1-\mu}<\frac{\mu}{1-3 \mu} \text { if and only if } 3 \mu^{2}-2 \mu+1>0,
$$

which is valid for every $\mu \in \mathbb{N}$. We proved with this the validity of 2.10 as desired.

We point out some characteristics of $g_{\lambda, \mu}$, which clarifies the behaviour of the iterates under the function for positive $\lambda$ 's.

If $x$ lies in $\left(1-\frac{1}{\mu}, 0\right)$, then it follows $g_{\lambda, \mu}(x)<x$. Analogously, $g_{\lambda, \mu}(x)>x$ for points satisfying $x<-\frac{1}{\mu}<x_{\mu}$. For every $x>x_{r}, x<g_{\lambda, \mu}(x)$. This follows directly from the fact that there is no fixed point larger than $x_{r}$, and $g_{\lambda, \mu}^{\prime}(x)>1$ for every $x \geq x_{r}$. We proceed to prove these properties in the following proposition.

Proposition 2.7. For $\lambda>0$ and $x<0$ we obtain one of the following

- For every $x \in\left(1-\frac{1}{\mu}, 0\right)$ it holds that $g_{\lambda, \mu}(x)<x$.

- For every $x \leq-\frac{1}{\mu}$, we have $x<g_{\lambda, \mu}(x)$.

Proof. For every negative $x$ we have $\lambda x e^{x}<0$. Such points satisfy as well that $(1-\mu)(x-1)<x$ if and only if $1-\frac{1}{\mu}<x$. Hence we obtain the first statement as

$$
g_{\lambda, \mu}(x)=(1-\mu)(x-1)+(1-\mu)\left(\lambda x e^{x}\right)<(1-\mu)(x-1)<x .
$$

We proved in Lemma 2.6 that the derivative $g_{\lambda, \mu}^{\prime}$ is positive for all $x \leq 2-\frac{1}{\mu}$ under the assumption that $\mu$ and $\lambda$ satisfy Inequality (2.4). Then $g_{\lambda, \mu}$ is increasing for every $x \leq-\frac{1}{\mu}<2-\frac{1}{\mu}$ and it suffices to prove the second statement for $x=-\frac{1}{\mu}$. It follows that

$$
-\frac{1}{\mu}<g_{\lambda, \mu}\left(-\frac{1}{\mu}\right)=(1-\mu)\left(-\frac{1}{\mu}-1-\lambda\left(\frac{1}{\mu}\right) e^{-\frac{1}{\mu}}\right)
$$

if and only if $-\mu<-\frac{1-\mu}{\mu} \lambda e^{-\frac{1}{\mu}}$ or equivalently if $\frac{\mu^{2}}{1-\mu}>\lambda e^{-\frac{1}{\mu}}$. The latter inequality follows using again Inequality (2.4). Thus we have

$$
\lambda e^{-\frac{1}{\mu}}<e^{\frac{1}{\mu}-2} \frac{\mu^{2}}{1-\mu} e^{-\frac{1}{\mu}}=e^{-2} \frac{\mu^{2}}{1-\mu},
$$

and it finally implies the claimed result in 2.11) from $e^{-2} \frac{\mu^{2}}{1-\mu}<\frac{\mu^{2}}{1-\mu}$. 
Considering $g_{\lambda, \mu}(z)$ again as a complex function (as we do in Section 2.3 and beyond) then the latter proposition imply that the interval $\left(x_{r}, \infty\right)$ is contained in the Julia set.

\subsubsection{Dynamics for $\lambda<0$}

In this subsection we show how the dynamics varies for different negative values of $\lambda$, such that the function $g_{\lambda, \mu}(x)$ has a unique critical point. Furthermore, $g_{\lambda, \mu}(x)$ has either one, two or three fixed points in the interval $\left(1-\frac{1}{\mu}, 0\right)$ following a bifurcation scheme.

Firstly, $g_{\lambda, \mu}$ has $x_{\mu}$ as unique attracting fixed point for $\lambda \in[-2,0)$. Secondly, there exists a $\lambda_{p} \in(-3,-2)$ such that $g_{\lambda, \mu}$ has a fixed point different from $x_{\mu}$. We denote it by $x_{p}$ and prove that it is a parabolic fixed point. Thirdly, $x_{p}$ bifurcates into an attracting fixed point, denoted by $x_{r}$, and a repelling fixed point, denoted by $x_{l}$, located respectively right and left from $x_{p}$. Fourthly, as $\lambda$ decreases forward below -4 , we find a second parameter $\lambda_{r}$ such that the attracting fixed point $x_{r}$ becomes parabolic. Finally, for further parameters $\lambda<\lambda_{r}, x_{r}$ is always repelling. The fixed point $x_{\mu}$ is always attracting and $x_{l}$ is repelling for all parameters $\lambda$ for which it exists.

Within this subsection, we consider $\mu \in\left(0, \frac{1}{5}\right)$ sufficiently small that Corollary 2.2 is satisfied, that is, $|\lambda|<e^{\frac{1}{\mu}-2} \frac{\mu^{2}}{1-\mu}$.

Theorem 2.8. For $\lambda<0$ there exist either one, two or three fixed points, all contained in the interval $\left(1-\frac{1}{\mu}, 0\right)$. The attracting fixed point $x_{\mu}$ is in the interval $\left(1-\frac{1}{\mu}, 2-\frac{1}{\mu}\right)$. If the additional fixed points exist, they are distributed as follows

i) For $\lambda \in[-2,0)$ the point $x_{\mu}$ is the only fixed point.

ii) There exists a $\lambda_{p} \in(-3,-2)$, such that $g_{\lambda, \mu}(x)$ has a parabolic fixed point, denoted by $x_{p}$.

iii) There exists $\lambda_{r}<-4$ such that for $\lambda_{r}<\lambda<\lambda_{p}$, the parabolic fixed point bifurcates into a repelling fixed point $x_{l}$ and an attracting fixed point $x_{r}$, with $x_{l}<x_{r}$.

iv) For $\lambda=\lambda_{r}<-4$ the attracting fixed point $x_{r}$ becomes parabolic.

v) For all $\lambda<\lambda_{r}$ both fixed points $x_{l}$ and $x_{r}$ (different from $x_{\mu}$ ) are repelling. 


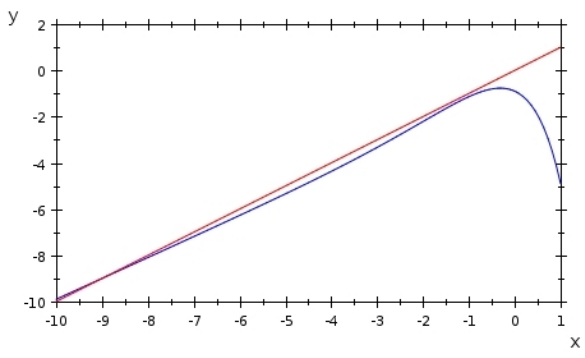

a) $\lambda=-2$

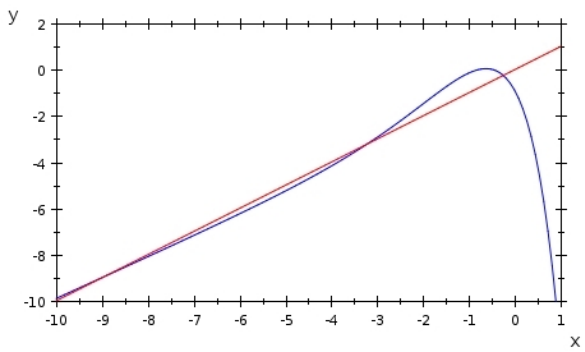

c) $\lambda=-5$

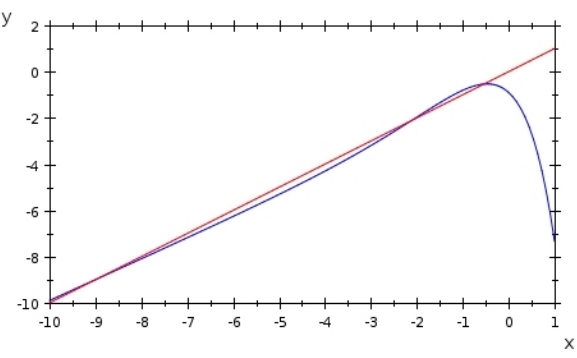

b) $\lambda=-3$

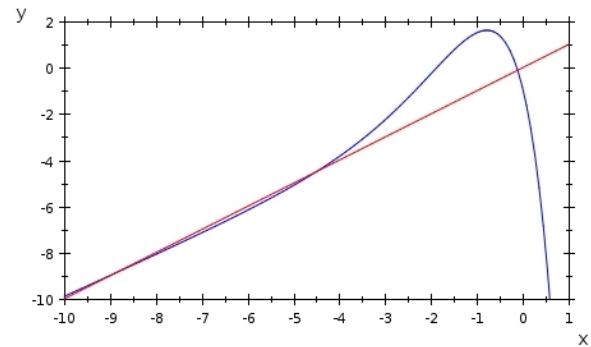

d) $\lambda=-10$

Figure 2.2: Graph of $g_{\lambda, \mu}(x)=(1-\mu)\left(x-1+\lambda x e^{x}\right)$ together with the identity map for $\mu=\frac{1}{10}$ and some values of $\lambda<0$. Figures show four different stages of the bifurcation scheme. In Figure a) there exists a unique fixed point $x_{\mu}$. Later in Figure b) the point $x_{p}$ bifurcated already into $x_{l}$ and $x_{r}$, one being repelling and the other attracting. Finally both points $x_{l}$ and $x_{r}$ are repelling in Figure c) and in Figure d).

Before we proceed with the proof of this theorem, we show some characteristics of $g_{\lambda, \mu}$ for negative $\lambda$, among them that there is a unique critical point. The proof of Theorem 2.8 follows immediately from Lemma 2.11 for the existence, and Lemmas 2.12 and 2.13 for the nature of the multipliers presented below. In Proposition 2.14 we show the computations for the approximated value of $\lambda_{p}$ and in Proposition 2.15 for $\lambda_{r}$.

Proposition 2.9. For $\lambda<0$ there exists exactly one critical point $c_{0}$, which is a global maximum for $g_{\lambda, \mu}$.

Proof. For negative $\lambda$ we have $g_{\lambda, \mu}^{\prime \prime}(x)=(1-\mu)\left(\lambda e^{x}\right)(x+2) \leq 0$ if and only if $x \in[-2, \infty)$. Therefore $g_{\lambda, \mu}^{\prime}(x)$ attains its maximum at $x=-2$. The value of the derivative at this point is $g_{\lambda, \mu}^{\prime}(-2)=(1-\mu)\left(1-\lambda e^{-2}\right)$, which is always positive. Recall that $g_{\lambda, \mu}^{\prime}(-1)>0$ for all $\lambda$ in $\mathbb{R}^{*}$ as showed in Equation 2.9. Furthermore 
$g_{\lambda, \mu}^{\prime}(x)=(1-\mu)\left(1+\lambda e^{x}(x+1)\right) \geq(1-\mu)$ for $x \leq-1$. Hence there are no critical points in the interval $(-\infty,-1]$.

Now, we separately consider the cases $x$ in $(-1,0)$ and $x \geq 0$. After some computations, we obtain that $g_{\lambda, \mu}^{\prime}(x)=0$ if and only if $\lambda e^{x}=\frac{-1}{x+1}$. On the one hand, write $h(x):=\frac{-1}{x+1}$ for the function on the right side of the latter equality. For $-1<x<0$ we have that $h(x)<-1$. If $x \geq 0$, then $-1 \leq h(x)<0$. On the other hand, the function $\lambda e^{x}$ on the left side is negative, decreasing for all $\lambda<0$. It equals $\lambda$ at $x=0$. Therefore if $\lambda<-1$ and $x \geq 0$ we obtain $\lambda e^{x} \leq \lambda<-1$. For these $\lambda$ 's the equality $\frac{-1}{x+1}=\lambda e^{x}$ has a solution only for $x<0$.

Concluding, the critical point $c_{0}$ is located as follows

i) For $-1<\lambda<0, c_{0}>0$.

ii) For $\lambda=-1$, the critical point $c_{0}$ equals 0 .

iii) For $\lambda<-1, c_{0} \in(-1,0)$.

At the beginning of the proof we showed that $g_{\lambda, \mu}^{\prime \prime}(x) \leq 0$ for $x \geq-2$. With this, we conclude that $c_{0} \in(-1, \infty)$ is a global maximum of $g_{\lambda, \mu}$.

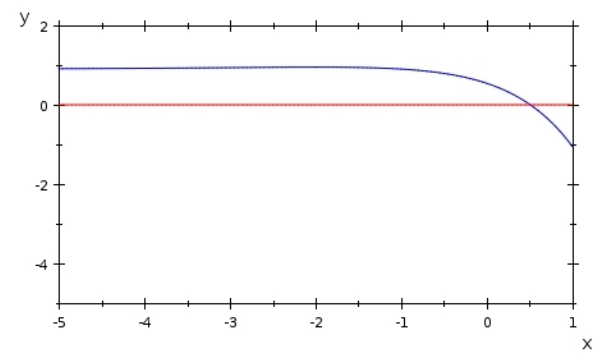

a) $\lambda=-0.4$

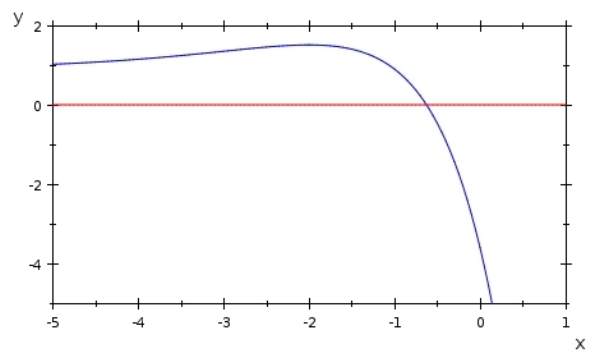

b) $\lambda=-5$

Figure 2.3: Graphs of the derivatives $g_{\lambda, \mu}^{\prime}(x)=(1-\mu)\left(1+\lambda x e^{x}+\lambda e^{x}\right)$ for $\mu=\frac{1}{10}$ and some values of $\lambda<0$. In Figure a) the critical point $c_{0}$ is positive. While in Figure b) $c_{0}$ lies in the interval $(-1,0)$.

Notice that for $\lambda<-1$ the derivative $g_{\lambda, \mu}^{\prime}(x)$ is negative for all $x \geq 0$, since $g_{\lambda, \mu}^{\prime}(0)=(1-\mu)(1+\lambda)$ comparing with Case (iii) above.

For any fixed point $x_{0}$ of $g_{\lambda, \mu}(x)$, we have that $\lambda e^{x_{0}}=\frac{\mu}{1-\mu}+\frac{1}{x_{0}}$. Making a 
substitution in its derivative $g_{\lambda, \mu}^{\prime}$ we obtain that

$$
\begin{aligned}
g_{\lambda, \mu}^{\prime}\left(x_{0}\right) & =(1-\mu)\left(1+\left(\frac{\mu}{1-\mu}+\frac{1}{x_{0}}\right)\left(x_{0}+1\right)\right) \\
& =(1-\mu)\left(2+\frac{1}{x_{0}}\right)+\mu\left(x_{0}+1\right) .
\end{aligned}
$$

We write

$$
\varphi(x):=(1-\mu)\left(2+\frac{1}{x}\right)+\mu(x+1)
$$

and study its behaviour, as it describes the values of the derivative at any fixed point.

Lemma 2.10. Let $\varphi(x)=(1-\mu)\left(2+\frac{1}{x}\right)+\mu(x+1)$ be a real function, with $0<\mu \leq \frac{1}{5}$. Then $\varphi(x)$ takes its local maximum at $x_{\varphi}=-\sqrt{1 / \mu-1}$ and for $x \in(-1,0)$ it holds $\varphi(x)<1$. Furthermore, for negative values of $x, \varphi(x) \rightarrow-\infty$ as $x$ approaches 0 .

Proof. The derivative $\varphi^{\prime}(x)=\frac{\mu-1}{x^{2}}+\mu$ equals zero if and only if $x_{1,2}= \pm \sqrt{\frac{1-\mu}{\mu}}$. Computing the second derivative $\varphi^{\prime \prime}(x)=\frac{2(1-\mu)}{x^{3}}$, we can see that it is positive for $x>0$ and negative for $x<0$, showing that $\varphi(x)$ attains a local maximum at the negative square root $x_{2}$ and a local minimum at the positive square root $x_{1}$. We now focus on values for $x<0$. Denote by

$$
x_{\varphi}=-\sqrt{\frac{1-\mu}{\mu}}<0
$$

the negative square root. The value at the maximum is:

$$
\begin{aligned}
\varphi\left(x_{\varphi}\right) & =(1-\mu)\left(2-\sqrt{\frac{\mu}{1-\mu}}\right)+\mu\left(1-\sqrt{\frac{1-\mu}{\mu}}\right) \\
& =2-\mu-2 \sqrt{\mu(1-\mu)} .
\end{aligned}
$$

Then $\varphi\left(x_{\varphi}\right)<2$ if and only if $\sqrt{\mu(1-\mu)}>-\frac{\mu}{2}$, which is true for all $0<\mu<1$. We now claim that $\varphi\left(x_{\varphi}\right) \geq 1$ for all $\mu \leq \frac{1}{5}$. After some computations the claim is equivalent to $5 \mu^{2}-6 \mu+1 \geq 0$ and holds for $\mu \leq \frac{1}{5}$ and $\mu \geq 1$. With this, we proved that $\varphi\left(x_{\varphi}\right) \in[1,2)$ for $\mu \in\left(0, \frac{1}{5}\right]$.

Hence for $x<0$ and $\mu<\frac{1}{5}$ there are exactly two points where the function $\varphi(x)$ attains the value 1 , more precisely at the points $x_{1,2}=\frac{\mu-1 \pm \sqrt{(1-\mu)(1-5 \mu)}}{2 \mu}$. 
Of particular interest is the fact that, for $x \in(-1,0)$, the function $\varphi(x)$ has values smaller than 1 and, as $x \rightarrow 0, \varphi(x) \rightarrow-\infty$. The first assertion is true because for $-1<x<0$ we obtain $\varphi(x)=(1-\mu)\left(2+\frac{1}{x}\right)+\mu(x+1)<(1-\mu)(2-1)+\mu=1$. The second assertion follows immediately for every $x<0$ from the inequality $\varphi(x)<\frac{(1-\mu)}{x}+2-\mu$.

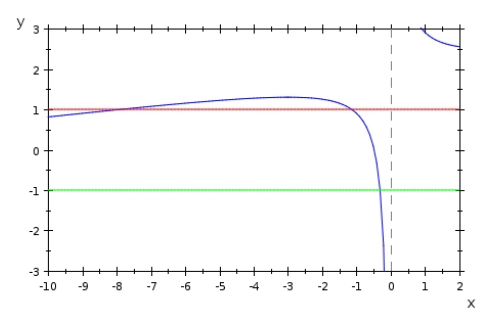

Figure 2.4: Graph of $g_{\lambda, \mu}^{\prime}\left(x_{0}\right):=\varphi\left(x_{0}\right)=(1-\mu)\left(2+\frac{1}{x_{0}}\right)+\mu\left(x_{0}+1\right)$ for any fixed point $x_{0}$ and $\mu=\frac{1}{10}$. The lines $y= \pm 1$ are shown in red and green respectively.

A solution for $\varphi(x)=1$ exists for $\mu \leq 1 / 5$ only. Because of this, we take values for $\mu$ always smaller or equal to $1 / 5$ within this subsection. Since the main interest of this work is an approximation process taking place in the limit as $\mu$ approaches 0 , this bound does not affect the final results.

Lemma 2.11. Let $\lambda<0$ and $\mu \in\left(0, \frac{1}{5}\right)$ be small enough that $|\lambda|<e^{\frac{1}{\mu}-2} \frac{\mu^{2}}{1-\mu}$. Then $g_{\lambda, \mu}(x)=(1-\mu)\left(x-1+\lambda x e^{x}\right)$ has either one, two or three fixed points as $\lambda$ decreases.

Proof. We proceed with the proof of the existence of the fixed points $x_{p}$ and later on $x_{l}$ and $x_{r}$ as $\lambda<0$ decreases. Analogously to the proof of Theorem 2.3, a point $x$ is fixed if and only if $\lambda e^{x}=\frac{\mu}{1-\mu}+\frac{1}{x}$. For negative parameters it follows that $\lambda e^{x}<0$. Then a fixed point can exist only if $\frac{\mu}{1-\mu}+\frac{1}{x}<0$ as well. This is true if and only if $1-\frac{1}{\mu}<x<0$.

We proved in Theorem 2.3 the existence of an attracting fixed point $x_{\mu}$ lying in the interval $\left(1-\frac{1}{\mu}, 2-\frac{1}{\mu}\right)$. Hence we consider only the points lying in the interval $\left(2-\frac{1}{\mu}, 0\right)$. A point $x$ is fixed if and only if it satisfies the equality $\lambda x e^{x}=\frac{\mu}{1-\mu} x+1$; see Figure 2.5. Denote the different sides of the equation by

$$
h(x)=\lambda x e^{x} \quad \text { and } \quad f(x)=\frac{\mu}{1-\mu} x+1 .
$$




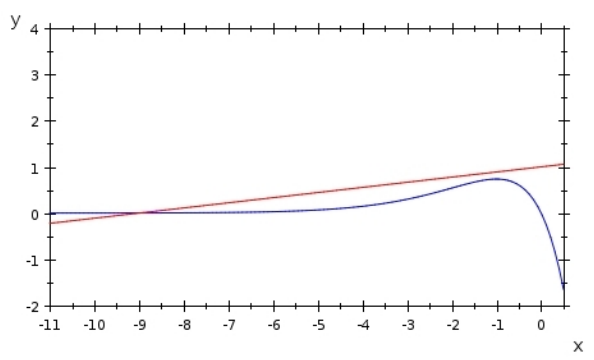

a) $\lambda=-2$

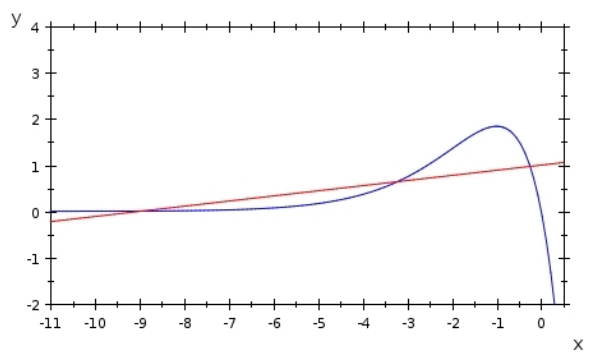

c) $\lambda=-5$

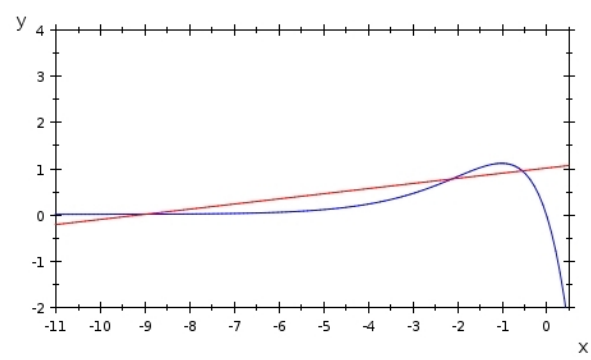

b) $\lambda=-3$

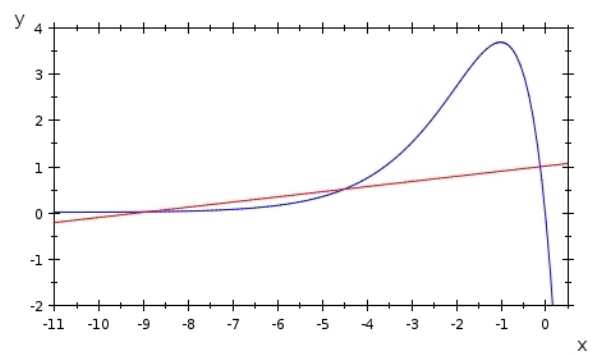

d) $\lambda=-10$

Figure 2.5: Functions $h(x)=\lambda x e^{x}$ for $\lambda<0$ and $f(x)=\frac{\mu}{1-\mu} x+1$ with $\mu=\frac{1}{10}$. Figures show the bifurcation scheme from the unique fixed point $x_{\mu}$ before the fixed point $x_{p}$ exists in a), into the existence of $x_{l}$ and $x_{r}$ in b) and the change from attracting into repelling nature of $x_{r}$ in c) and d) as $\lambda$ decreases.

On the one hand, the function $h(x)$ has its global maximum at $x=-1$, since it is the unique zero of $h^{\prime}(x)=\lambda e^{x}(x+1)$ and its second derivative $h^{\prime \prime}(x)=\lambda e^{x}(x+2)$ is negative at $x=-1$ for all $\lambda<0$. Notice that $h(x) \geq 0$ for all $x \leq 0$. For $x>0$, $h(x) \rightarrow-\infty$ and therefore $f(x)$ and $h(x)$ cannot be equal.

On the other hand, we can see that $f(x)$ is a linear function with positive slope and unique zero at $x=1-\frac{1}{\mu}$. Furthermore, for every $\mu \leq \frac{1}{5}$ the value $f(-1)=2-\frac{1}{1-\mu}$ lies in $\left[\frac{3}{4}, 1\right)$. Hence the value of $h(x)$ at $x=-1$ will determine the existence of fixed points of $g_{\lambda, \mu}$.

Consider $x \leq 0$. Firstly, we prove that $h\left(2-\frac{1}{\mu}\right)<f\left(2-\frac{1}{\mu}\right)$ with $|\lambda|<$ $e^{\frac{1}{\mu}-2} \frac{\mu^{2}}{1-\mu}$. On the one hand,

$$
f\left(2-\frac{1}{\mu}\right)=\frac{\mu}{1-\mu}\left(\frac{2 \mu-1}{\mu}\right)+1=\frac{\mu}{1-\mu}
$$


On the other hand,

$$
h\left(2-\frac{1}{\mu}\right)=\lambda\left(2-\frac{1}{\mu}\right) e^{2-\frac{1}{\mu}}<e^{\frac{1}{\mu}-2} \frac{\mu^{2}}{1-\mu}\left(\frac{2 \mu-1}{\mu}\right) e^{2-\frac{1}{\mu}}=\frac{\mu(2 \mu-1)}{1-\mu} .
$$

Finally, comparing both values we obtain

$$
\frac{\mu(2 \mu-1)}{1-\mu}<\frac{\mu}{1-\mu} \text { if and only if } \quad \mu<1 .
$$

Secondly, we see that $h(-1)=-\lambda e^{-1}>0$ for all $\lambda<0$ and that $h$ increases as $\lambda$ decreases to $-\infty$. Furthermore, for $-2 \leq \lambda<0$ we have $h(-1) \leq 2 e^{-1}<\frac{3}{4}$, implying that $h(-1)<\frac{3}{4} \leq f(-1)$. Since $h^{\prime}(x)$ is always increasing for $x<-1$, there is no intersection of $f(x)$ and $h(x)$ in the interval $\left(2-\frac{1}{\mu}, 0\right)$ for $\lambda \in[-2,0)$.

Thirdly, for $\lambda \leq-3$ the value $h(-1) \geq 3 e^{-1}>1$. Then $h(-1)>f(-1)$ and since both functions are strictly increasing for $x<-1$, we can conclude that there exists an $x_{l} \in\left(2-\frac{1}{\mu},-1\right)$ such that $h\left(x_{l}\right)=f\left(x_{l}\right)$. For $x \in(-1,0), f(x)$ lies in the interval $\left[\frac{3}{4}, 1\right)$ and is increasing, while $h(x)$ decreases from $-\lambda e^{-1}$ to 0 . Hence there again exists a point $x_{r} \in(-1,0)$ such that $h\left(x_{r}\right)=f\left(x_{r}\right)$.

Denote by $x_{p}$ a point such that $h^{\prime}\left(x_{p}\right)=\frac{\mu}{1-\mu}$. Since $h^{\prime}\left(x_{p}\right)>0$, then $x_{p}<$ -1 and $h\left(x_{p}\right)<h(-1)$. Following the argumentation from previous paragraph, for parameters $\lambda \leq-3$ the functions $h(x)$ and $f(x)$ intersect twice, in contrast to parameters $\lambda \in[-2,0)$ where there exists no such an intersection. Then by continuity, there must exist a $\lambda_{p} \in(-3,-2)$ such that $f(x)$ is tangent to $h(x)$. Since $f(x)$ is a linear function with slope $\frac{\mu}{1-\mu}$, the tangency point is precisely $x_{p}$.

Lemma 2.12. The point $x_{p}$ is a parabolic fixed point of $g_{\lambda, \mu}(x)$, while $x_{l}$ is always a repelling fixed point.

Proof. By definition of the point $x_{p}, h^{\prime}\left(x_{p}\right)=\lambda e^{x_{p}}\left(x_{p}+1\right)=\frac{\mu}{1-\mu}$. Using this equality in the computation of the multiplier, the first assertion follows from $g_{\lambda, \mu}^{\prime}\left(x_{p}\right)=(1-\mu)\left(1+\frac{\mu}{1-\mu}\right)=1-\mu+\mu=1$.

The point $x_{l}$ is a solution to the equality $g_{\lambda, \mu}(x)=x$. Recall that $g_{\lambda, \mu}$ has a unique critical point $c_{0}>-1$ which is a global maximum. Furthermore, for values of $\lambda$ smaller than $\lambda_{p}$, we have that $x_{l}<x_{p}<-1$. Hence $1=g_{\lambda, \mu}^{\prime}\left(x_{p}\right)<g_{\lambda, \mu}^{\prime}(x)$ for $x \in\left[x_{l}, x_{p}\right)$. 
Lemma 2.13. The point $x_{r}$ is an attracting fixed point of $g_{\lambda, \mu}(x)$ for parameters $\lambda$ between $\lambda_{r}$ and $\lambda_{p}$. For the latter parameter $x_{p}$ is a parabolic fixed point and for all $\lambda<\lambda_{r}, x_{r}$ is repelling.

Proof. We proved in the previous lemma, that $g_{\lambda, \mu}^{\prime}\left(x_{p}\right)=1$. For $\lambda<\lambda_{p}$ and using Lemma 2.11, we have that $x_{p}<x_{r}$. As $\lambda$ decreases, the maximum value of the function $h(x)$ increases, and with this, the point $x_{r}$ increases from $x_{p}$ above -1 and approaches 0 . Hence there exists a $\lambda_{0}<\lambda_{p}$ such that $x_{r}$ is in the interval $(-1,0)$ for all $\lambda \leq \lambda_{0}$.

Using now the derivative function for fixed points $\varphi(x)$ as defined in Lemma 2.10 . it holds that $\varphi\left(x_{p}\right)=1>\varphi\left(x_{r}\right)$. With this we have that $x_{r}$ is an attracting fixed point for parameters $\lambda \in\left(\lambda_{p}-\varepsilon, \lambda_{p}\right)$, for some $\varepsilon>0$. We proved in Lemma 2.10 that if $x \in(-1,0)$, then $\varphi(x)<1$ always. In particular, $x_{r}$ remains attracting as it increases above -1 . Even more, we proved that $\varphi(x) \rightarrow-\infty$ as $x \rightarrow 0$. Then, there exists a parameter $\lambda_{r}$ such that $\varphi\left(x_{r}\right)=-1$ and for further $\lambda<\lambda_{r}$, $\varphi\left(x_{r}\right)<-1$, meaning that the fixed point $x_{r}$ is repelling for all parameters below $\lambda_{r}$.

The parameters $\lambda$ for which the fixed points $x_{p}$ or $x_{l}$ and $x_{r}$ exist are smaller than -2 , as we proved in Lemma 2.11. This implies that the critical point $c_{0}$ is in $(-1,0)$ and approaches -1 as $\lambda$ decreases toward $-\infty$.

Of particular interest is the maximum value of $g_{\lambda, \mu}$, that is $g_{\lambda, \mu}\left(c_{0}\right)$, since it tends to infinity as $c_{0}$ approaches -1 . From Equation (2.6), we obtain that $c_{0}$ is a critical point if and only if $\lambda e^{c_{0}}=\frac{-1}{c_{0}+1}$. Hence its value is given by

$$
g_{\lambda, \mu}\left(c_{0}\right)=(1-\mu)\left(c_{0}-2+\frac{1}{c_{0}+1}\right) .
$$

It follows that $g_{\lambda, \mu}\left(c_{0}\right)$ has an asymptotic point at $c_{0}=-1$ and that $g_{\lambda, \mu}\left(c_{0}\right) \geq 0$ for $c_{0} \in(-1,-1+\varepsilon]$ and some $0<\varepsilon<\frac{1}{2}$.

Now, we present some computations to approximate the value $\lambda_{r} \approx-3 e^{1 / 3}$, that is, the parameter $\lambda_{r}$ for which the fixed point $x_{r}$ is parabolic after being attracting and before it becomes repelling.

Proposition 2.14. Let $x_{r}$ be a fixed point contained in $(-1,0)$. Then the parameter $\lambda_{r}$ for which $x_{r}$ is parabolic satisfies $\lambda_{r}<-3 \exp \left(\frac{1-\mu}{3-2 \mu}\right)$ and tends to $-3 e^{1 / 3}$ as $\mu$ tends to zero.

Proof. We proved in Lemma 2.13 that the fixed point $x_{r}$ is at first attracting, later parabolic and finally repelling as $\lambda$ decreases below -3 . We also showed that $x_{r}$ 
is in the interval $(-1,0)$ if $\varphi\left(x_{r}\right)=-1$. Therefore we restrict the point $x$ within this proof to this interval.

Notice that for $x \in(-1,0)$ the function $\varphi(x)$ is decreasing. We also have that $\varphi(x)=(1-\mu)\left(2+\frac{1}{x}\right)+\mu(x+1)>(1-\mu)\left(2+\frac{1}{x}\right)$ and the latter equals -1 if $x=\frac{1-\mu}{-3+2 \mu}$ which is negative. Since $x_{r}$ is a fixed point, it satisfies the equation $\lambda e^{x_{r}}=\frac{1}{x_{r}}+\frac{\mu}{1-\mu}$. It also holds that $x_{r}>-\frac{1-\mu}{3-2 \mu}>\frac{-1}{3}$ for all $\mu$ and with this

$$
\lambda_{r}=e^{-x_{r}}\left(\frac{1}{x_{r}}+\frac{\mu}{1-\mu}\right)<e^{\frac{1-\mu}{3-2 \mu}}\left(\frac{-3+2 \mu}{1-\mu}+\frac{\mu}{1-\mu}\right)=-3 e^{\frac{1-\mu}{3-2 \mu}} .
$$

We finalise with $\lambda_{r}<-3 e^{1 / 3} \approx-4.1868$, and tends to this value as $\mu \rightarrow 0$.

We gave in Lemma 2.11 a qualitative proof for the existence of a parameter $\lambda_{p} \in(-3,-2)$ such that $g_{\lambda, \mu}$ has a fixed point $x_{p}$ different from $x_{\mu}$ and which is parabolic. This qualitative proof was helpful to show the dynamical behaviour for negative parameters $\lambda$ and the bifurcation scheme presented by the fixed points $x_{p}, x_{l}$ and $x_{r}$. Here we present a numerical approximation for the parameter $\lambda_{p}$.

Proposition 2.15. Let $x_{p}$ be a fixed point contained in $\left(2-\frac{1}{\mu},-1\right)$. Then the parameter $\lambda_{p}$ for which $x_{p}$ is parabolic satisfies $\lambda_{p}>\left(-1+\frac{2 \mu}{1-\mu}\right) \exp \left(1+\frac{\mu}{1-2 \mu}\right)$ and tends to $-e$ as $\mu$ tends to zero.

Proof. Assume the fixed point $x_{p}<-1$ is parabolic with multiplier $\varphi\left(x_{p}\right)=1$. Then it holds that $\varphi(x)=(1-\mu)\left(2+\frac{1}{x}\right)+\mu(x+1)<(1-\mu)\left(2+\frac{1}{x}\right)$ since $\mu(x+1)<0$. From this we obtain

$$
(1-\mu)\left(2+\frac{1}{x}\right)=1 \quad \text { if and only if } \quad x=\frac{1-\mu}{2 \mu-1} .
$$

Since $x_{p}$ is a fixed point, we may compute $\lambda$ for $x_{p}<\frac{1-\mu}{2 \mu-1}$ as in previous lemma

$$
\begin{aligned}
\lambda_{p}=e^{-x_{p}}\left(\frac{1}{x_{p}}+\frac{\mu}{1-\mu}\right) & >e^{\frac{1-\mu}{1-2 \mu}}\left(\frac{-1+2 \mu}{1-\mu}+\frac{\mu}{1-\mu}\right) \\
& =\left(-1+\frac{2 \mu}{1-\mu}\right) e^{1+\frac{\mu}{1-2 \mu}},
\end{aligned}
$$

where the last expression converges to $-e$ from above as $\mu \rightarrow 0$.

Using the approximation for $\lambda$ in the two previous propositions, we can say that for points $x_{r}$ in the interval $\left(-1-\frac{\mu}{1-2 \mu},-\frac{1-\mu}{3-2 \mu}\right), x_{r}$ is an attracting fixed point of 
the function $g_{\lambda, \mu}(x)$. The result in the following proposition implies in particular that the basin of attraction of $x_{r}$ is small and remains close to the origin. The study of the family $g_{\lambda, \mu}$ on the complex plane in the next sections will provide a more complete description of the dynamics and the structure of its Fatou set.

Proposition 2.16. Let $\lambda<0$. Then the function $g_{\lambda, \mu}(x)$ has no fixed points outside of the interval $\left[1-\frac{1}{\mu}, 0\right]$.

Proof. We prove the statement by showing that for $x<1-\frac{1}{\mu}$ we have $x<g_{\lambda, \mu}(x)$ and for all $x>0$ then $g_{\lambda, \mu}(x)<x$. Furthermore, if $\lambda \leq-1$ we obtain that $g_{\lambda, \mu}(x)<0$ for all $x \geq 0$.

On the one hand, we have for $x<1-\frac{1}{\mu}<0$ that $x<(1-\mu)(x-1)$ together with $\lambda x e^{x}>0$. Hence it immediately follows that

$$
x<(1-\mu)(x-1)<(1-\mu)(x-1)+(1-\mu)\left(\lambda x e^{x}\right)=g_{\lambda, \mu}(x) .
$$

On the other hand, if $x>0$, then $\lambda x e^{x}<0$. In particular we obtain that $x>1-\frac{1}{\mu}$ for a fixed point. In this case we have

$$
x>(1-\mu)(x-1)>(1-\mu)(x-1)+(1-\mu)\left(\lambda x e^{x}\right)=g_{\lambda, \mu}(x) .
$$

Finally we show that $g_{\lambda, \mu}(x)<0$ for every $x \geq 0$ and $\lambda \leq-1$. We notice that $g_{\lambda, \mu}^{\prime}(0)=(1-\mu)(1+\lambda)<0$ if and only if $\lambda \leq-1$. We prove that for such $\lambda$ 's, the critical point $c_{0}$ lies in $(-1,0]$. Then $g_{\lambda, \mu}^{\prime}(x) \leq 0$ for all $x \geq 0$. Hence it immediately follows that $g(x)<g_{\lambda, \mu}(0)=\mu-1<0$.

\subsection{Singular points and values of $g_{\lambda, \mu}$}

In the following, consider again $g_{\lambda, \mu}(z)=(1-\mu)\left(z-1+\lambda z e^{z}\right)$ as a complex function with $\lambda \in \mathbb{C}^{*}$ and $\mu \in\left(0, \frac{1}{2}\right)$. It is known that the critical points are points where the inverse map cannot be uniquely defined. For entire functions this is equivalent to finding the zeros of the derivative. In addition to critical points, entire transcendental functions may have asymptotic values.

In this chapter we firstly prove that $g_{\lambda, \mu}$ has no asymptotic values for every $\lambda \in \mathbb{C}^{*}$ and every $\mu \in(0,1)$. Then we construct a domain $\mathcal{M}_{c, \mu}$ such that the critical points lie on its boundary and derive some of its properties. Recall that the set of critical points of $g_{\lambda, \mu}$ equals the set of critical points of $g_{\lambda}(z)=z-1+\lambda z e^{z}$. Hence for completeness we include some results due to Lauber [48] concerning the approximated location of critical points originally made for $g_{\lambda}$ but which apply 
for $g_{\lambda, \mu}$ as well. To conclude we present some results about the location of critical values of $g_{\lambda, \mu}$.

Proposition 2.17. Let $\lambda \in \mathbb{C}^{*}$ and $\mu \in\left(0, \frac{1}{2}\right)$. Then $g_{\lambda, \mu}(z)=(1-\mu)\left(z-1+\lambda z e^{z}\right)$ has no asymptotic values.

Proof. Assume that $g_{\lambda, \mu}$ has at least one asymptotic value $a$. Then there exists a Jordan arc $\gamma(t)$ with $t \in[0, \infty)$ such that

$$
\lim _{t \rightarrow \infty}|\gamma(t)|=\infty \quad \text { and } \quad \lim _{t \rightarrow \infty} g_{\lambda, \mu}(\gamma(t))=a
$$

Then

$$
\begin{aligned}
\frac{\lim _{t \rightarrow \infty} g_{\lambda, m}(\gamma(t))}{\lim _{t \rightarrow \infty} \gamma(t)} & =\lim _{t \rightarrow \infty} \frac{(1-\mu)\left(\gamma(t)-1+\lambda \gamma(t) e^{\gamma(t)}\right)}{\gamma(t)} \\
& =\lim _{t \rightarrow \infty}(1-\mu)\left(1-\frac{1}{\gamma(t)}+\lambda e^{\gamma(t)}\right) \\
& =0
\end{aligned}
$$

if and only if

$$
1-\mu=\lim _{t \rightarrow \infty}(1-\mu)\left(\frac{1}{\gamma(t)}-\lambda e^{\gamma(t)}\right)
$$

which implies that $1=-\lambda \lim _{t \rightarrow \infty} e^{\gamma(t)}$, a contradiction for any fixed $\lambda \in \mathbb{C}^{*}$.

Now we concentrate on the properties and location of the critical points and values of $g_{\lambda, \mu}$. The derivative of $g_{\lambda, \mu}$ is given by:

$$
g_{\lambda, \mu}^{\prime}(z)=(1-\mu)\left(1+\lambda e^{z}(z+1)\right) .
$$

For every $z \neq-1$ the derivative equals zero if and only if:

$$
\lambda e^{z}=\frac{-1}{z+1} \quad \text { or equivalently } \quad \lambda z e^{z}=-1-\lambda e^{z} .
$$

Let $c \neq-1$ be a critical point. The last two equations imply that each critical value $v$ can be described by the expression:

$$
v:=g_{\lambda, \mu}(c)=(1-\mu)\left(c-2+\frac{1}{c+1}\right) .
$$

Recall that the main goal of this work is to study the perturbation of the function

$$
g_{\lambda}(z)=z-1+\lambda z e^{z} \text { with } \quad \lambda \in \mathbb{C}^{*} .
$$


It is easy to see that the critical points are preserved by the perturbation, since the zeros of the derivative $g_{\lambda}^{\prime}(z)=1+\lambda e^{z}(z+1)$ remain invariant. However the invariance is not fulfilled by the critical values of $g_{\lambda, \mu}$, which are contracted in direction to the origin by $1-\mu$ in comparison to the critical values of $g_{\lambda}$. There are infinitely many critical points, since zero is not a Piccard's omitted value of $g_{\lambda, \mu}^{\prime}$. Furthermore we have that following result.

Corollary 2.18. The set of critical points of $g_{\lambda, \mu}$ is unbounded.

The idea of the proof is based on the fact that the set of critical points of $g_{\lambda}$ remains invariant under the perturbation $g_{\lambda, \mu}$. Since the Fatou set of $g_{\lambda}$ contains a Baker domain, the proof follows from a result of Eremenko and Lyubich stated as Theorem 1.11 in the introduction.

Another property preserved by the perturbation of $g_{\lambda}$ is revealed by the construction of the domain $\mathcal{M}_{c, \mu}$. All critical points $c$ of $g_{\lambda, \mu}(z)$ lie on the boundary of $\mathcal{M}_{c, \mu}$, where

$$
\begin{aligned}
\mathcal{M}_{c, \mu} & :=\left\{z \in \mathbb{C}:\left|g_{\lambda, \mu}^{\prime}(z)-(1-\mu)\right|<1-\mu\right\} \\
& =\left\{z \in \mathbb{C}:\left|(1-\mu)\left(\lambda e^{z}\right)(z+1)\right|<1-\mu\right\}
\end{aligned}
$$

since the equality is achieved when $g_{\lambda, \mu}^{\prime}(z)=0$. The domain $\mathcal{M}_{c, \mu}$ coincides with an analogue domain in the dynamical plane of the function $g_{\lambda}$ constructed by Lauber in [48. The critical points of $g_{\lambda}$ lie on the boundary of the domain defined as $\mathcal{M}_{c}=\left\{z \in \mathbb{C}:\left|g_{\lambda}^{\prime}(z)-1\right|<1\right\}=\left\{z \in \mathbb{C}:\left|\left(\lambda e^{z}\right)(z+1)\right|<1\right\}$. In accordance with the definition, all points $z \in \mathcal{M}_{c, \mu}$ satisfy that $g_{\lambda, \mu}^{\prime}(z)$ lies in the disc $D(1-$ $\mu, 1-\mu)$ and for $z \in \partial \mathcal{M}_{c, \mu}$ it follows that $g_{\lambda, \mu}^{\prime}(z) \in \partial D(1-\mu, 1-\mu)$.

In both cases we have the equivalent expression for the domains:

$$
\mathcal{M}_{c}=\mathcal{M}_{c, \mu}=\left\{z=x+i y: y^{2}<\frac{e^{-2 x}}{|\lambda|^{2}}-(x+1)^{2}\right\} .
$$

The latter equation implies that the domain $\mathcal{M}_{c, \mu}$ does not dependent on the parameter $\mu$. In other words, it is also invariant under the perturbation.

Proposition 2.19. (adapted from Lauber, [48], Lemma 14) $\mathcal{M}_{c, \mu}$ is non-empty, symmetric with respect to the real axis, consists of at most two components and exactly one component is unbounded. If there are two components, then the bounded one is contained in the strip $\{z \in \mathbb{C}:|\operatorname{Im} z| \leq 1 / 2\}$. If $z \in \mathcal{M}_{c, \mu}$ and $\operatorname{Im} z>1 / 2$, then $x+i y \in \mathcal{M}_{c, \mu}$ for all $x$, $y$ satisfying $x<\operatorname{Re} z,|y|<\operatorname{Im} z$. Furthermore, if $x \in\left(\mathbb{C} \backslash \mathcal{M}_{c, \mu}\right) \cap(-1, \infty)$, then $\mathcal{M}_{c, \mu} \subset\{z: \operatorname{Re} z<x\}$.

Proof. Having Equation (2.16) in mind, we write $h(x):=\frac{e^{-2 x}}{|\lambda|^{2}}-(x+1)^{2}$. Then $h^{\prime}(x)=\frac{-2 e^{-2 x}}{|\lambda|^{2}}-2(x+1)$ equals zero if and only if $\frac{e^{-2 x}}{|\lambda|^{2}}=-(x+1)$. Hence, a critical 
point $x$ of $h(x)$ satisfies $h(x)=-(x+1)-(x+1)^{2} \leq \frac{1}{4}$ for all $x \in \mathbb{R}$. Furthermore, $h^{\prime \prime}(x)=\frac{4 e^{-2 x}}{|\lambda|^{2}}-2$ has at most one root, and, consequently, there are at most two roots of $h^{\prime}$. Hence $h$ has at most one maximum value and one minimum value which are smaller than $1 / 4$. The maximum and minimum are smaller than -1 , since $h^{\prime}(x)<0$ for all $x>-1$. Finally, $h(x) \rightarrow+\infty$ as $x \rightarrow-\infty$.

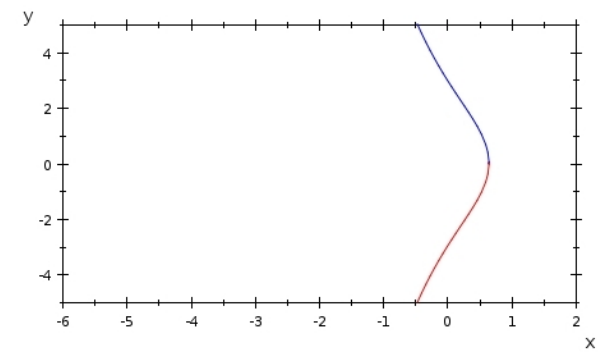

a) $|\lambda|=0.1$

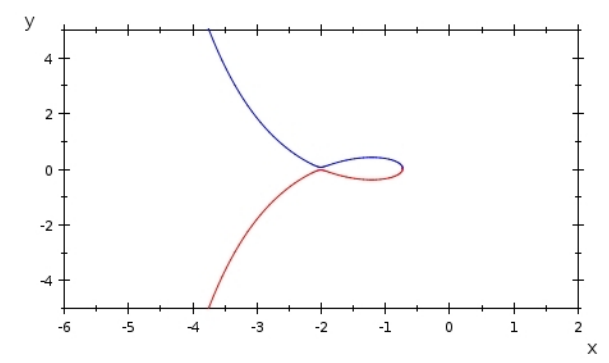

c) $|\lambda|=e^{2}$

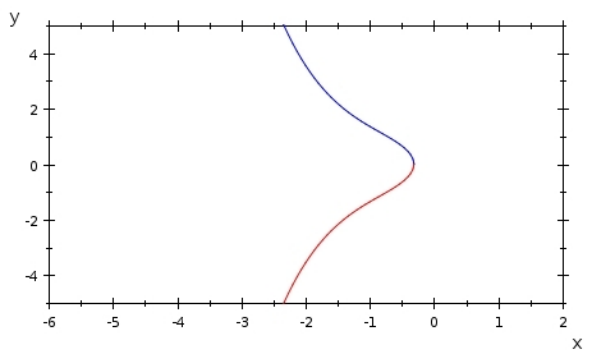

b) $|\lambda|=2$

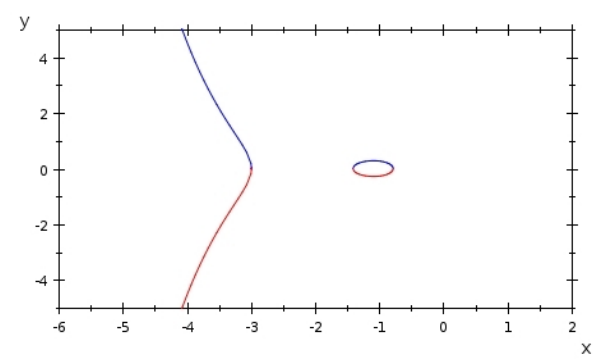

d) $|\lambda|=10$

Figure 2.6: Boundary of domain $\mathcal{M}_{c, \mu}=\left\{x+i y \in \mathbb{C}: y^{2}<\frac{e^{-2 x}}{|\lambda|^{2}}-(x+1)^{2}\right\}$ for some values of $\lambda$ where the critical points of $g_{\lambda, \mu}$ lie on.

The definition of the domain $\mathcal{M}_{c, \mu}$ depends on $|\lambda|$, from which we can say that $\partial \mathcal{M}_{c, \mu}$ is symmetric with respect to the real axis for every $\lambda \in \mathbb{C}^{*}$. This does not imply the same statement for the critical points. Notice that $\lambda e^{\bar{z}}=\overline{\lambda e^{z}}$ if and only if $\lambda=\bar{\lambda}$. Hence, the functions $g_{\lambda, \mu}$ and $g_{\lambda, \mu}^{\prime}$ map points with real axis symmetry in the sense that $g_{\lambda, \mu}(\bar{z})=\overline{g_{\lambda, \mu}(z)}$ (resp. for $g_{\lambda, \mu}^{\prime}$ ), if and only if $\lambda \in \mathbb{R}^{*}$.

Therefore we may state the results for a critical point $c$ with $\operatorname{Im}(c)>\varepsilon$ for some $\varepsilon>0$ and they will follow for $c^{\prime}$ with $\operatorname{Im}\left(c^{\prime}\right)<-\varepsilon$. Following the same line of argumentation, we may state the results for $\lambda$ with $\operatorname{Im} \lambda \geq 0$ without loss of generality, and they are analogously satisfied by $\bar{\lambda}$.

Remark 2.1. Critical points depend holomorphically on $\lambda$ for any arbitrary but fixed $\mu \in\left(0, \frac{1}{2}\right)$. Notice that $z$ is a critical point if and only if $\lambda=\frac{-e^{-z}}{z+1}$. The 
function $h(z)=\frac{-e^{-z}}{z+1}$ maps critical points of $g_{\lambda, \mu}$ to the parameter $\lambda$. Since $h^{\prime}(z)=$ $\frac{e^{-z}(z+2)}{(z+1)^{2}}$, the singularities of the inverse function of $h$ are the critical value $e^{2}$ and the asymptotic value 0 . Then all inverse branches of $h$ are well defined and holomorphic in the domain $\mathbb{C}^{*} \backslash \mathbb{R}^{+}$, and each of these branches maps the parameter value $\lambda$ to one of the critical points of the corresponding function $g_{\lambda, \mu}$.

Recall that for $\lambda \in \mathbb{R}^{-}$the real line contains exactly one critical point of $g_{\lambda, \mu}$ in contrast to parameters $\lambda \in \mathbb{R}^{+}$, where the function may have none, one or two real critical points. Because of this, some of the results stated within this and the following chapter are valid for parameters $\lambda \in \mathbb{C}^{*} \backslash \mathbb{R}^{+}$.

Lemma 2.20. Let $\lambda \in \mathbb{C}^{*}$, with $\lambda \neq e^{2}$, then all critical points are simple.

Proof. A critical point $c$ is simple if $f^{\prime}(c)=0$ implies $f^{\prime \prime}(c) \neq 0$. We prove the lemma by showing that if a critical point is not simple, then $\lambda=e^{2}$. We have on the one hand that $g_{\lambda, \mu}^{\prime \prime}(c)=(1-\mu)\left(\lambda e^{c}\right)(c+2)$ equals zero if and only if $c=-2$. On the other hand $c=-2$ is a critical point exactly for $\lambda=e^{2}$, since $g_{\lambda, \mu}^{\prime}(-2)=(1-\mu)\left(1-\lambda e^{-2}\right)$.

Following the representation of $\partial \mathcal{M}_{c, \mu}$ in Equation 2.16), we label the critical points $c_{k}$ for $k \in \mathbb{Z}^{*}$ after their distribution with respect to their imaginary part.

Labelling. Let $k \in \mathbb{Z}$. We label the critical points as follows.

- For $\lambda \in \mathbb{R}^{*}$ we denote by $c_{0}$ the real critical point if it is unique, and $c_{01}, c_{02}$ in case there are two real critical points; see Section 2.2.

- In general, for $\lambda \in \mathbb{C}^{*}$ and $k=0$ it holds that $\operatorname{Im} c_{0} \in(-\pi, \pi)$.

- For a critical point with $\operatorname{Im} c_{k}>0$ we use an index $k>0$. Analogously negative values of $k$ are used when $\operatorname{Im} c_{k}<0$.

- The general order is given by $\operatorname{Im} c_{k+1}>\operatorname{Im} c_{k}$ for $k \in \mathbb{Z}$.

In the following proposition we show that the labelling is well defined and convenient to give an estimated position for the critical points. Some tools used in the proof of Theorem 2.21 will be presented in form of lemmas and the proof itself is written directly after these lemmas. In the sense of Remark 2.1 recall that the critical points $c_{k}=c_{k}(\lambda)$ depend on $\lambda$. But for convenience we omit this dependence in the labelling.

Theorem 2.21 (Lauber, Lemma 16 [48]). Let $\lambda \in \mathbb{C}^{*} \backslash \mathbb{R}^{+}$and $c_{k}$ be a critical point with $k \in \mathbb{Z}$. Then $c_{k}$ has the following properties.

i) $\operatorname{Im} c_{k}>\pi$ for $k>0$ and $\operatorname{Im} \lambda \geq 0$. 
ii) $\operatorname{Im} c_{k}<0$ for $k<0$.

iii) $\operatorname{Im} c_{0}>0$ for $\operatorname{Im} \lambda>0$.

iv) $\operatorname{Im} c_{0} \in(-\pi, \pi)$.

v) $\operatorname{Im} c_{k}>\operatorname{Im} c_{j}$ if $k>j$.

vi) $\left|\operatorname{Im} c_{k}-\operatorname{Im} c_{k+1}\right|$ and $\left|c_{k}-c_{k+1}\right|$ tend to $2 \pi$ as $|k|$ tends to $\infty$.

Lemma 2.22 (adapted from Lauber, [48, Lemma 24). Let $\operatorname{Im} \lambda>0$ and $c_{k}$ be a critical point with $\operatorname{Im} c_{k} \in\left(-\frac{\pi}{2}, 0\right)$. Then $\operatorname{Re} c_{k}<-1$ and $\operatorname{Re} g_{\lambda, \mu}\left(c_{k}\right)<\operatorname{Re} c_{k}-2$.

Proof. Let $\lambda=a+i b$ with $b>0$. We write

$$
\begin{aligned}
g_{\lambda, \mu}^{\prime}(z) & =(1-\mu)\left(1+\lambda(z+1) e^{z}\right) \\
& =(1-\mu)\left(1+(a+i b)(x+1+i y) e^{x}(\cos y+i \sin y)\right) \\
& =(1-\mu)\left(1+e^{x}\left(a \alpha_{1}-b \alpha_{2}\right)+i e^{x}\left(a \alpha_{2}+b \alpha_{1}\right)\right)
\end{aligned}
$$

with

$$
\begin{aligned}
& \alpha_{1}=(x+1) \cos y-y \sin y \quad \text { and } \\
& \alpha_{2}=(x+1) \sin y+y \cos y .
\end{aligned}
$$

Let $c_{k}=x_{k}+i y_{k}$ with $y_{k} \in(-\pi / 2,0)$. Since $c_{k}$ is a critical point, it holds that $\operatorname{Im} g_{\lambda, \mu}^{\prime}\left(c_{k}\right)=(1-\mu) e^{x_{k}}\left(a \alpha_{2}+b \alpha_{1}\right)=0$. Assume that $x_{k}>-1$, which implies that $\alpha_{2}<0$. Firstly, if we assume that $a \alpha_{1}<0$ together with $b>0$, then we obtain $a \alpha_{2}+b \alpha_{1} \neq 0$. Secondly, for $a \alpha_{1}>0$ it follows that $\operatorname{Re} g_{\lambda, \mu}^{\prime}\left(x_{k}+i y_{k}\right)=$ $(1-\mu)\left(1+e^{x}\left(a \alpha_{1}+b \alpha_{2}\right)\right)>1-\mu$. Thirdly, if $a \alpha_{1}=0$, then we obtain also that $\operatorname{Re} g_{\lambda, \mu}^{\prime}\left(x_{k}+i y_{k}\right)>1-\mu$. In the three cases above, the assumptions lead to a contradiction to the fact that $c_{k}$ is a critical point. Finally, if $x_{k}=-1$, then we obtain that $\alpha_{1}$ and $\alpha_{2}$ are non zero. Hence we obtain that $x_{k}<-1$ must be satisfied as claimed.

The proof of the second statement follows directly from Equation 2.15), since $\operatorname{Re} g_{\lambda, \mu}\left(c_{k}\right)=(1-\mu)\left(x_{k}-2+\frac{x_{k}+1}{\left|c_{k}+1\right|^{2}}\right)<x_{k}-2$ for every $x_{k}<-1$.

Lemma 2.23 (adapted from Lauber, Lemmas 16 and 25 [48]). Let $\lambda=a+i b$. We obtain the following negative results.

i) For $\lambda \in \mathbb{R}^{-}$the function $g_{\lambda, \mu}$ has no critical points in the strips $\{z \in \mathbb{C}: 0<$ $\operatorname{Im} z \leq \pi\}$ and $\{z \in \mathbb{C}:-\pi \leq \operatorname{Im} z<0\}$.

ii) For $\lambda \in \mathbb{C}^{*}$ with $b \geq 0$, the function $g_{\lambda, \mu}$ has no critical points on the line $\{z=x+i y: y=\pi\}$. 
iii) For $\lambda \in \mathbb{C}^{*}$ with $b \geq 0$, the function $g_{\lambda, \mu}$ has no critical points on the line $\{z=x+i y: y=-2 \pi\}$.

Proof. We prove the first statement by using the notation from Lemma 2.22 . Write $\lambda=a+i b$ with $a<0$ and $b=0$. Then we have

$$
g_{\lambda, \mu}^{\prime}(x+i y)=(1-\mu)\left(1+a e^{x}\left(\alpha_{1}+i \alpha_{2}\right)\right)
$$

The imaginary part of $g_{\lambda, \mu}^{\prime}$ equals zero if and only if $\alpha_{2}=(x+1) \sin y+y \cos y=0$. For $y \in(0, \pi]$ the equality holds for $(x+1) \cos y<0$. The latter inequality together with the assumption $a<0$ imply $\operatorname{Re} g_{\lambda, \mu}^{\prime}(x+i y)=(1-\mu)\left(1+a e^{x} \alpha_{1}\right)>1-\mu$. Hence the existence of a critical point under such conditions is not possible. Symmetry arguments yield to the same conclusion for $z=x+i y$ with $y \in[-\pi, 0)$.

For proving the second statement, let $z=x+i \pi$ with $x \in \mathbb{R}$ and $\lambda=a+i b$ with $b \geq 0$. Then $|z| \neq 0$. We assume that $z$ is a critical point. Then $g_{\lambda, \mu}^{\prime}(x+i \pi)=0$ if and only if $\lambda=\frac{-e^{-z}}{z+1}=\frac{-e^{-(x+i \pi)}}{x+1+i \pi}=e^{-x} \frac{x+1-i \pi}{|x+1+i \pi|^{2}}$. The latter equality implies in particular that $\operatorname{Im} \lambda=\frac{-i \pi e^{-x}}{|x+1+i \pi|^{2}}<0$, leading to a contradiction.

The third statement is proved in a similar way. Let $z=x-i 2 \pi$ be a critical point and $b \geq 0$. Then $g_{\lambda, \mu}^{\prime}(x-i 2 \pi)=(1-\mu)\left(1+\lambda(x+1-i 2 \pi) e^{x-i 2 \pi}\right)=0$ if and only if

$$
\lambda=\frac{-e^{-x}}{x+1-i 2 \pi}=e^{-x}\left(\frac{-x-1}{|x+1-i 2 \pi|^{2}}-\frac{i 2 \pi}{|x+1-i 2 \pi|^{2}}\right) .
$$

Then it must hold that $\operatorname{Im} \lambda=b<0$, proving our statement.

Lemma 2.24 (adapted from Lauber, Lemma 25, [48]). There exists a $\lambda \in \mathbb{R}^{-}$ such that $\operatorname{Im} c_{1}>\pi$ and $\operatorname{Im} c_{-2}<-2 \pi$.

Proof. As we proved in Lemma 2.23 there exist no critical points in the strips of height $\pi$ above and below the real axis. Hence $\operatorname{Im} c_{1}>\pi$ and $\operatorname{Im} c_{-1}<-\pi$.

For parameters $\lambda$ with sufficiently small norm we may assume that the curve $\gamma:=\partial \mathcal{M}_{c, \mu} \cap\{z \in \mathbb{C}:-2 \pi \leq \operatorname{Im} z<-\pi\}$ is close to a segment of length $\pi$. In particular its length is less than $\frac{3 \pi}{2}$. Furthermore, $\gamma \subset\{z \in \mathbb{C}: \operatorname{Re} z>-2\}$ holds also for $|\lambda|$ sufficiently small. Hence $g_{\lambda, \mu}^{\prime \prime}(z)=(1-\mu)\left(\lambda(z+2) e^{z}\right) \neq 0$ on $\gamma$ and 
using our definition of $\mathcal{M}_{c, \mu}$ we obtain for points $z \in \gamma$

$$
\begin{aligned}
\left|g_{\lambda, \mu}^{\prime \prime}(z)\right| & =\left|(1-\mu)\left(\lambda(z+1) e^{z}+\lambda e^{z}\right)\right| \\
& \leq(1-\mu)\left|\lambda(z+1) e^{z}\right|+(1-\mu)\left|\lambda e^{z}\right| \\
& =(1-\mu)\left|\lambda(z+1) e^{z}\right|+\frac{1-\mu}{|z+1|} \\
& =(1-\mu)\left(1+\frac{1}{|z+1|}\right) \leq(1-\mu)\left(1+\frac{1}{\pi}\right)<1+\frac{1}{\pi}<\frac{4}{3} .
\end{aligned}
$$

Since $g_{\lambda, \mu}^{\prime}(z) \in \partial D(1-\mu, 1-\mu)$, this implies that there is at most one critical point contained in $\gamma$, which is the point $c_{-1}$. Hence $\operatorname{Im} c_{-2}<-2 \pi$.

Proof of Theorem 2.21. The proof of the first statement in the theorem follows directly from the computations in Lemma 2.23 , that is, that $\operatorname{Im} c_{0}<\pi$ for parameters $\operatorname{Im} \lambda \geq 0$. In particular, we proved that $\operatorname{Im} c_{k}>\pi$ for $k>0$ and $\operatorname{Im} \lambda \geq 0$.

Next if $x$ is a real critical point, then $\lambda$ is real as well. Since in this case $\lambda$ is restricted to $\mathbb{R}^{-}$, then $c_{0}$ is the unique critical point on the real line and we have $\operatorname{Im} c_{k}<0$ for $k<0$. This proves statement $\left.i i\right)$.

To prove the third statement, that is $\operatorname{Im} c_{0}>0$ for $\operatorname{Im} \lambda>0$, it suffices to show that there exists a parameter in the upper half-plane such that the line $\frac{i \pi}{2}$ contains a critical point. Let $x \in \mathbb{R}$. The parameter $\lambda=\frac{-e^{-x-i \pi / 2}}{x+1+i \pi / 2}$ satisfies

$$
g_{\lambda, \mu}^{\prime}\left(x+i \frac{\pi}{2}\right)=(1-\mu)\left(1-\frac{e^{-x-i \pi / 2}}{x+1+i \pi / 2}(x+1+i \pi / 2) e^{x+i \pi / 2}\right)=0 .
$$

Then $z=x+i \frac{\pi}{2}$ must to be the critical point $c_{0}$ using statements $i$ ) and $i i$ ). Hence $c_{0}$ is contained in the strip bounded by the real axis and the horizontal line at $i \pi$ when $\operatorname{Im} \lambda>0$.

Using symmetry arguments, the statement above also follows for $\lambda$ with $\operatorname{Im} \lambda<0$, which applies that $c_{0}$ is always contained in the strip $\{z \in \mathbb{C}:-\pi<\operatorname{Im} z<\pi\}$ for every $\lambda \in \mathbb{C}^{+} \backslash \mathbb{R}^{+}$, yielding result $\left.i v\right)$.

To show the fifth statement, let $\operatorname{Im} \lambda \geq 0$. Firstly, assume that $\left|\operatorname{Im} c_{k}\right|>\frac{1}{2}$. Then we consider critical points which lie in the boundary of the unbounded component of $\mathcal{M}_{c, \mu}$, which is the union of the graphs of two continuous monotonous functions as we proved in Lemma 2.19. Note that two critical points never collide for a parameters outside from $\mathbb{R}^{+}$, otherwise some function $c_{k}(\lambda)$ would not be analytic at this parameter. Since all critical points move along the boundary of $\mathcal{M}_{c, \mu}$, 
which itself moves continuously with respect to $\lambda$, we obtain that $\operatorname{Im} c_{k}>\operatorname{Im} c_{j}$ for $k>j$ and for critical points outside the strip $\{z \in \mathbb{C}:|\operatorname{Im} z|<1 / 2\}$. Now, inside the strip we may meet the difficulty that the boundary of $\mathcal{M}_{c, \mu}$ is not longer the graph of a monotonous function for every parameter $\lambda$. But we know that $\operatorname{Im} c_{k}>0$ if $k>0, \operatorname{Im} c_{1}>\pi$ and $\operatorname{Im} c_{-1}<0$ as proved in Lemmas 2.23 and 2.24. So it remains to show that $\operatorname{Im} c_{-2}<-2 \pi$. This is true for $\lambda \in \mathbb{R}^{-}$as shown in Lemma 2.24. But we also showed that the line $-2 \pi i$ does not contain any critical point if $\operatorname{Im} \lambda \geq 0$, which yields the claim.

To prove the last statement, recall that $\partial \mathcal{M}_{c, \mu}$ is mapped by $g_{\lambda, \mu}^{\prime}$ into the boundary of the disc $D(1-\mu, 1-\mu)$ and contains all critical points. It suffices to show that $\left|g_{\lambda, \mu}^{\prime \prime}(z)\right|$ tends to $1-\mu$ for $z \in \partial \mathcal{M}_{c, \mu}$ as $|z|$ tends to $\infty$. We compute

$$
\begin{aligned}
\left|g_{\lambda, \mu}^{\prime \prime}(z)\right| & =(1-\mu)\left|\lambda(z+1) e^{z}+\lambda e^{z}\right| \\
& \leq(1-\mu)\left(\left|\lambda(z+1) e^{z}\right|+\left|\lambda e^{z}\right|\right)=(1-\mu)\left(1+\frac{1}{|z+1|}\right),
\end{aligned}
$$

which under these conditions tends to $1-\mu$.

We now present some estimates for the critical values.

Proposition 2.25. Let $c \in \mathbb{C}$ be a critical point different from -1 and denote its value by $v=g_{\lambda, \mu}(c)$. If $\operatorname{Im} c \neq 0$, then $|\operatorname{Im} v|<|\operatorname{Im} c|$. Furthermore, for critical points with $|\operatorname{Im} c|>2$ we obtain that the critical value $v \in D\left((1-\mu)(c-2), \frac{1}{2}\right)$.

Proof. Firstly, recall that any critical value satisfies Equation 2.15, which is equivalent to $v=(1-\mu)\left(c-2+\frac{\bar{c}+1}{|c+1|^{2}}\right)$. Then for $c=x+i y$ we obtain that $\operatorname{Im} v<y$ if and only if

$$
-\mu y-\frac{(1-\mu) y}{|c+1|^{2}}<0 .
$$

The latter inequality holds for every $y>0$ and $\mu>0$. Analogously, we obtain $y<\operatorname{Im} v$ for every $y<0$.

Secondly, to prove that $v \in D\left((1-\mu)(c-2), \frac{1}{2}\right)$ we again use Equation 2.15). From the assumption that $|\operatorname{Im} c|>2$ we have that $2<|\operatorname{Im}(c+1)| \leq|c+1|$. Then $|v-(1-\mu)(c-2)|=(1-\mu)\left|\frac{1}{c+1}\right|<\left|\frac{1}{c+1}\right|<\frac{1}{2}$, which yields the desired result.

Proposition 2.26 (adapted from Lauber, 48, Lemma 22). Let $c_{k}=x_{k}+i y_{k}$ be a critical point with $y_{k}>0$ and $\lambda \in \mathbb{C}^{*}$. Then the following properties hold.

i) For every $x<x_{k}$ it is satisfied $\operatorname{Im} g_{\lambda, \mu}\left(x_{k}+i y_{k}\right)<\operatorname{Im} g_{\lambda, \mu}\left(x+i y_{k}\right)<y_{k}$ and $\frac{\partial}{\partial x} \operatorname{Im} g_{\lambda, \mu}\left(x+i y_{k}\right)<0$. 
ii) Denote by $L=\left\{x+i y_{k}: x<x_{k}\right\}$. If $L \subset \mathcal{M}_{c, \mu}$ or $x_{k}<-1$, then all $z \in L$ with $x<x_{k}$ satisfy $\operatorname{Re} g_{\lambda, \mu}\left(x+i y_{k}\right)<\operatorname{Re} g_{\lambda, \mu}\left(x_{k}+i y_{k}\right)$.

iii) If $x_{k}>-1$, then $\operatorname{Re} g_{\lambda, \mu}\left(x+i y_{k}\right)<\operatorname{Re} g_{\lambda, \mu}\left(x_{k}+i y_{k}\right)$ for all $x \in\left[-2, x_{k}\right)$.

Proof. Let $\lambda=a+i b$ and $z=x+i y$. During this proof we write the product $\lambda e^{z}:=e^{x}\left(\rho_{1}+i \rho_{2}\right)$ whereby

$$
\begin{aligned}
& \rho_{1}=a \cos y-b \sin y \quad \text { and } \\
& \rho_{2}=a \sin y+b \cos y
\end{aligned}
$$

Then we write

$$
\begin{aligned}
& g_{\lambda, \mu}(x+i y)=(1-\mu)\left(x-1+i y+e^{x}\left(x \rho_{1}-y \rho_{2}\right)+i e^{x}\left(x \rho_{2}+y \rho_{1}\right)\right) \quad \text { and } \\
& g_{\lambda, \mu}^{\prime}(x+i y)=(1-\mu)\left(1+e^{x}\left((x+1) \rho_{1}-y \rho_{2}\right)+i e^{x}\left((x+1) \rho_{2}+y \rho_{1}\right)\right)
\end{aligned}
$$

using the notation above. Notice that the functions $\rho_{1}$ and $\rho_{2}$ depend on $y$ and $\lambda$ only.

We now proceed to prove the first statement. As we have shown in Proposition 2.25. $\operatorname{Im} g_{\lambda, \mu}\left(c_{k}\right)<y_{k}$ for every $k \neq 0$, then $\operatorname{Im} g_{\lambda, \mu}\left(x_{k}+i y_{k}\right)-y_{k}<0$. Denote by $h(x)$ the function defined for every $x \in \mathbb{R}$ and the fixed value $y_{k}$ as

$$
h(x)=\operatorname{Im} g_{\lambda, \mu}\left(x+i y_{k}\right)-y_{k}=-\mu y_{k}+(1-\mu) e^{x}\left(x \rho_{2}+y_{k} \rho_{1}\right) .
$$

Then $h^{\prime}(x)=(1-\mu) e^{x}\left((x+1) \rho_{2}+y_{k} \rho_{1}\right)$ coincides with $\operatorname{Im} g_{\lambda, \mu}^{\prime}\left(x+i y_{k}\right)$. This implies that $h^{\prime}(x)$ has exactly one root at $x_{k}$. Since $h\left(x_{k}\right)$ is negative by definition and $h(x)$ tends to $-\mu y_{k}<0$ as $x \rightarrow-\infty$, we conclude that $h(x)<0$ for $x<x_{k}$. Furthermore, we obtain for all $x<x_{k}$

$$
h^{\prime}(x)=\frac{\partial\left(\operatorname{Im} g_{\lambda, \mu}^{\prime}\left(x+i y_{k}\right)\right)}{\partial x}<(1-\mu) e^{x_{k}}\left(\left(x_{k}+1\right) \rho_{2}+y_{k} \rho_{1}\right)=0 .
$$

Then $h\left(x_{k}\right)<h(x)$, which yields the statement.

In order to prove the second property, assume first that $L \subset \mathcal{M}_{c, \mu}$. Then $g_{\lambda, \mu}^{\prime}(z)$ lies in the disc $D(1-\mu, 1-\mu)$ for all $z \in L$. We have then for such $z$ that $\operatorname{Re} g_{\lambda, \mu}^{\prime}(z)$ lies in the interval $(0,2(1-\mu))$. Hence $0=\operatorname{Re} g_{\lambda, \mu}^{\prime}\left(c_{k}\right)<\operatorname{Re} g_{\lambda, \mu}^{\prime}(z)$ and $\operatorname{Re} g_{\lambda, \mu}\left(x+i y_{k}\right)$ is an increasing function of $x$. Finally $\operatorname{Re} g_{\lambda, \mu}(z)<\operatorname{Re} g_{\lambda, \mu}\left(c_{k}\right)$ for all $x<x_{k}$.

Assume now that $x_{k}<-1$ and $y_{k}>0$. Furthermore, assume that $\rho_{1}<0$. Then $\operatorname{Im} g_{\lambda, \mu}^{\prime}\left(c_{k}\right)=(1-\mu) e^{x_{k}}\left(\left(x_{k}+1\right) \rho_{2}+y_{k} \rho_{1}\right)=0$ implies $\rho_{2}<0$ and this gives $\operatorname{Re} g_{\lambda, \mu}^{\prime}\left(c_{k}\right)=(1-\mu)\left(1+e^{x_{k}}\left(\left(x_{k}+1\right) \rho_{1}-y_{k} \rho_{2}\right)\right)>(1-\mu)\left(1+e^{x_{k}}\right)>1-\mu$, 
which leads to a contradiction for a critical point $c_{k}$. We conclude that $\rho_{1} \geq 0$ and $\rho_{2}>0$ must hold, thus we obtain

$$
\begin{aligned}
\operatorname{Re} g_{\lambda, \mu}\left(x+i y_{k}\right) & =(1-\mu)\left(x-1+e^{x}\left(x \rho_{1}+y_{k} \rho_{2}\right)\right) \\
& <(1-\mu)\left(x_{k}-1+e^{x_{k}}\left(x_{k} \rho_{1}+y_{k} \rho_{2}\right)=\operatorname{Re} g_{\lambda, \mu}\left(c_{k}\right)\right.
\end{aligned}
$$

for all $x<x_{k}$. In other words, $\operatorname{Re} g_{\lambda, \mu}(z)<\operatorname{Re} g_{\lambda, \mu}\left(c_{k}\right)$ holds also for critical points with $x_{k}<-1$ and $z \in L$.

We finally prove the last statement in a similar way as above. Let $x_{k}>-1$ and assume $\rho_{1}>0$. Again, $\operatorname{Im} g_{\lambda, \mu}^{\prime}\left(c_{k}\right)=0$ implies $\rho_{2}<0$ and $\operatorname{Re} g_{\lambda, \mu}^{\prime}\left(c_{k}\right)>(1-\mu)$, which is a contradiction to $c_{k}$ being a critical point. Hence we have that $\rho_{1} \leq 0$ and $\rho_{2}>0$. Beside this, $\operatorname{Re} g_{\lambda, \mu}^{\prime \prime}\left(x+i y_{k}\right)=(1-\mu) e^{x}\left((x+2) \rho_{1}-y_{k} \rho_{2}\right)<0$ for $-2 \leq x<x_{k}$. Then $\operatorname{Re} g_{\lambda, \mu}^{\prime}\left(x+i y_{k}\right)>\operatorname{Re} g_{\lambda, \mu}^{\prime}\left(x_{k}+i y_{k}\right)=0$ for $x \in\left[-2, x_{k}\right)$, as desired.

Corollary 2.27. Let $c_{k}$ be a critical point and $v_{k}$ its value for every $k \in \mathbb{Z}^{*}$. If $\operatorname{Im} c_{k}<\operatorname{Im} c_{j}$, then $\operatorname{Im} v_{k}<\operatorname{Im} v_{j}$ and $\left|c_{k}\right|<\left|c_{j}\right|$. In particular, the result is valid for $j=k+1$.

This result follows immediately from Theorem 2.21 and Proposition 2.25. As a conclusion we mention that if $|k| \geq 1$ it follows from the properties of the critical points that $\left|\operatorname{Im} c_{k}\right|>\pi>2$ implying that $v_{k} \in D\left((1-\mu)\left(c_{k}-2\right), \frac{1}{2}\right)$ for all such points.

\subsection{Absorbing domain $\mathcal{H}_{\eta, \rho}$}

In Section 2.1 we have shown the existence of an attracting fixed point $z_{\mu}$ for the function $g_{\lambda, \mu}$. In order to give a first description of the basin of attraction of $z_{\mu}$, $\mathcal{A}_{\mu}$, we construct an absorbing domain contained in it. The aim of this work is to prove the dynamical convergence of the functions $g_{\lambda, \mu}$ to $g_{\lambda}$. One way of proving this is to show the convergence of their Fatou components. Therefore we prove later in Section 3.3 that the basin of attraction $\mathcal{A}_{\mu}$ converges to the Baker domain $\mathcal{B}_{\lambda}$ using the convergence of the absorbing domains.

A first suggestion is an appropriate variation of the absorbing domain for the Baker domain defined as $\mathcal{M}_{\delta}=\left\{z \in \mathbb{C}:\left|g_{\lambda}(z)-(z-1)\right|<\delta\right\}$ for $\delta \in(0,1)$. Hence we construct an analogous domain $\mathcal{M}_{\delta, \mu}=\left\{z \in \mathbb{C}:\left|g_{\lambda, \mu}(z)-(1-\mu)(z-1)\right|<\delta\right\}$, presented below in Subsection 2.4.1. The geometry of the domain $\mathcal{M}_{\delta, \mu}$ is preserved by the approximating functions $g_{\lambda, \mu}$, but it is not invariant under these functions. 
One of the limitations presented by the action of $g_{\lambda, \mu}$ is the multiplicative factor $1-\mu$. If we multiply any point $z$ by $1-\mu$, then it is contracted in direction of the straight line connecting the point $z$ with the origin by a factor of its norm. Notice that points $z \in \mathcal{M}_{\delta, \mu}$ are mapped very close to $(1-\mu)(z-1)$. Therefore, $z$ is firstly mapped close to $z-1$ and secondly contracted in direction to the origin. Consequently, points with large norm and lying close to the boundary of $\mathcal{M}_{\delta, \mu}$ may be mapped out of the domain after the first iteration. In fact, the dynamics for points with very large imaginary part have a very intricate dynamic as we can see in Figure 2.7 .

However, the intricate dynamic depends strongly on the parameter $\mu$ and vanishes up to infinity in the direction of the imaginary axis as $\mu$ tends to zero. Hence we firstly restrict the domain $\mathcal{M}_{\delta, \mu}$ to a subset denoted by $\mathcal{M}_{\rho}$, which is its intersection with the right half-plane $\left\{z \in \mathbb{C}: \operatorname{Re} z>1-\frac{1}{2 \mu}\right\}$. Furthermore, for points $z=x+i y$ with $x \leq\left(1-\frac{1}{2 \mu}\right)$ we define a domain $\mathcal{H}_{\eta} \subset \mathcal{M}_{\delta, \rho}$.

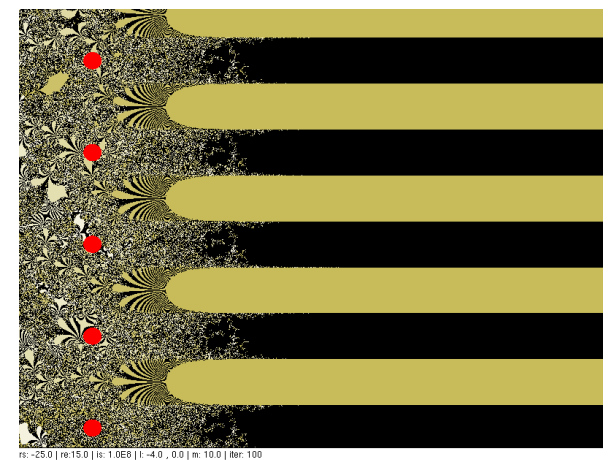

a) $\operatorname{Im} z \approx 10^{8}, \lambda=-4$ and $\mu=\frac{1}{10}$.

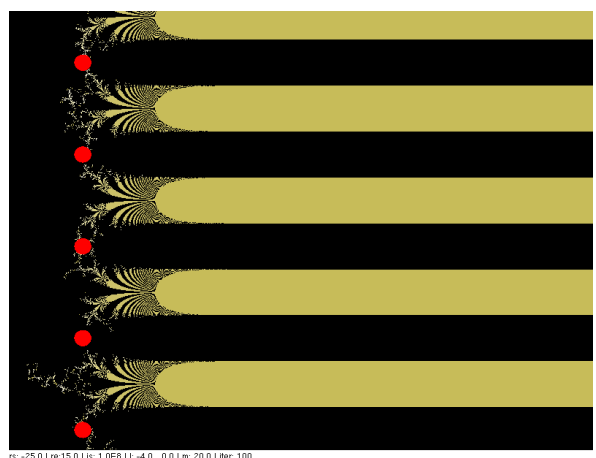

b) $\operatorname{Im} z \approx 10^{8}, \lambda=-4$ and $\mu=\frac{1}{20}$.

Figure 2.7: Fatou and Julia sets of $g_{\lambda, \mu}(z)=(1-\mu)\left(z-1+\lambda z e^{z}\right)$ for points with large imaginary part. The back region belongs to the basin of attraction $\mathcal{A}_{\mu}$, each red circle is the neighbourhood of a critical point and $\mathcal{J}\left(g_{\lambda, \mu}\right)$ is in yellow. As $\mu$ tends to zero, the "chaotic" behaviour of $\mathcal{J}\left(g_{\lambda, \mu}\right)$ shrinks upwards and downwards $\infty$ in the $i \mathbb{R}$ direction and disappears in the limit.

In Subsection 2.4.1 we present some geometrical properties of the domain $\mathcal{M}_{\delta, \mu}$. In Subsection 2.4.2 we construct the domain $\mathcal{H}_{\eta, \rho}=\mathcal{H}_{\eta} \cup \mathcal{M}_{\rho}$ and prove its invariance under the function $g_{\lambda, \mu}$. 


\subsubsection{First approach by $\mathcal{M}_{\delta, \mu}$}

In this subsection we present the geometrical description of the domain $\mathcal{M}_{\delta, \mu}$. In the following, the value of $\mu$ is sufficiently small that the attracting fixed point $z_{\mu}$ exists as required in Theorem 2.1.

For every $\lambda \in \mathbb{C}^{*}$ we define the domain $\mathcal{M}_{\delta, \mu}$, with $\mu \in\left(0, \frac{1}{2}\right), \mu \rightarrow 0$ and $\delta \in(0,1)$ as

$$
\mathcal{M}_{\delta, \mu}=\left\{z \in \mathbb{C}:\left|g_{\lambda, \mu}(z)-(1-\mu)(z-1)\right|<\delta\right\}
$$

Since $\left|g_{\lambda, \mu}(z)-(1-\mu)(z-1)\right|=\left|(1-\mu)\left(\lambda z e^{z}\right)\right|$, we obtain after some computations that the domain $\mathcal{M}_{\delta, \mu}$ can equivalently be described as

$$
\mathcal{M}_{\delta, \mu}=\left\{z=x+i y \in \mathbb{C}: y^{2}<\left(\frac{\delta}{(1-\mu)|\lambda|}\right)^{2} e^{-2 x}-x^{2}\right\} .
$$

The equivalence between the two definitions is independent of the parameters $\lambda$, $\mu$ and $\delta$. Furthermore, with a change of variable $x \mapsto t+1$ we can rewrite the domain as

$$
\mathcal{M}_{\delta, \mu}=\left\{z=t+1+i y \in \mathbb{C}: y^{2}<\left(\frac{\delta e^{-1}}{(1-\mu)}\right)^{2} \frac{e^{-2 t}}{|\lambda|^{2}}-(t+1)^{2}\right\} .
$$

Hence, $\mathcal{M}_{\delta, \mu}$ is just a translation by one to the left of the domain $\mathcal{M}_{c, \mu}$ described in the previous section with the Equation 2.16). We may neglect the constant, since it does not depend on $\lambda$ and tends to $\delta e^{-1} \approx 1$ as $\mu$ tends to zero. We obtain the following result.

Proposition 2.28. The domain $\mathcal{M}_{\delta, \mu}$ is non-empty, symmetric with respect to real axis, consists of at most two connected components of which exactly one is unbounded. If there are two components, the bounded one is contained in the strip $\{z \in \mathbb{C}:|\operatorname{Im} z|<1 / 2\}$. Furthermore $\partial \mathcal{M}_{\delta, \mu}$ can be parametrized as a function of $x$ for $x \leq \sigma_{\mu}:=\min \left\{-e, 2 \ln \left(\frac{\delta}{(1-\mu)|\lambda|}\right)\right\}$.

In the following proofs we use some properties of $\ln x$. For $x>0$ we have

i) $1-\frac{1}{x} \leq \ln x \leq x-1$.

ii) $\ln x<\frac{x}{2}$.

The first statement follows immediately from the equality at $x=1$ and the definition of $\ln x$. The second statement is valid for all $x \in(0, \infty)$. Notice its equivalence to $x<e^{x / 2}$ and to $x^{2}<e^{x}$, which in turn holds for positive values of $x$. 


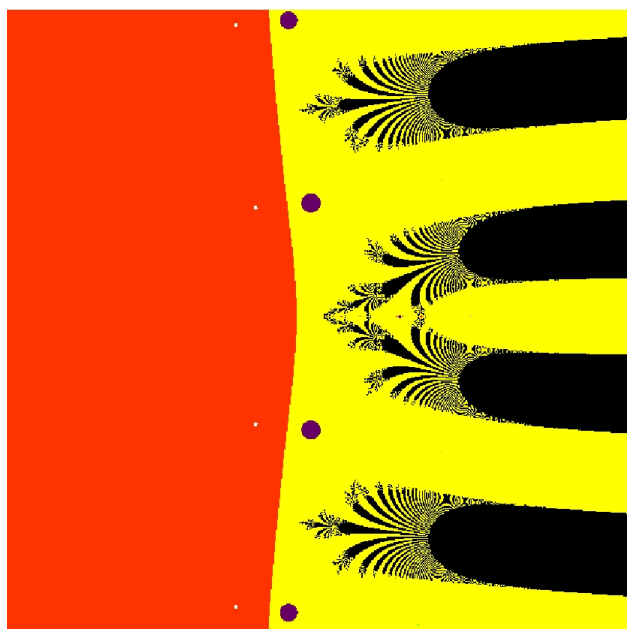

Figure 2.8: Absorbing domain $\mathcal{M}_{\delta, \mu}$ for $\delta=\frac{1}{2}, \mu=\frac{1}{100}$ and $\lambda=-10$ in orange contained in a left half-plane. Critical points and values are shown in dark blue and white circles, resp. The yellow part belongs to the basin of attraction $\mathcal{A}_{\mu}$ and the Julia set $\mathcal{J}\left(g_{\lambda, \mu}\right)$ is in black.

Proof. The symmetry of $\mathcal{M}_{\delta, \mu}$, the number of its components and their properties are inherited from the domain $\mathcal{M}_{c, \mu}$ as proved in Lemma 2.19. Now, the boundary of $\mathcal{M}_{\delta, \mu}$ is described by the graph of the function $h_{\delta, \mu}$, that is, as the set of points $\left\{(x, y): y= \pm h_{\delta, \mu}(x)\right\}$ with

$$
h_{\delta, \mu}(x):=\sqrt{\left(\frac{\delta}{(1-\mu)|\lambda|}\right)^{2} e^{-2 x}-x^{2}} .
$$

We compute the upper boundary for the values $x \in \mathbb{R}$ such that $h_{\delta, \mu}$ is well defined. The discriminant of the function $h_{\delta, \mu}$ is positive if and only if

$$
\begin{aligned}
|x| e^{x} & \leq \frac{\delta}{(1-\mu)|\lambda|} \quad \text { or equivalently } \\
\ln |x|+x & \leq \ln \left(\frac{\delta}{(1-\mu)|\lambda|}\right)
\end{aligned}
$$

For $x \leq-e$ it follows $x+\ln |x|<x+\frac{1}{2}|x|=\frac{x}{2}$. We define

$$
\sigma_{\mu}:=\min \left\{-e, 2 \ln \left(\frac{\delta}{(1-\mu)|\lambda|}\right)\right\}
$$


and assume that $x \leq \sigma_{\mu}$. Then for $\sigma_{\mu}=-e$ the result follows immediately from the arguments presented above. Otherwise we obtain

$$
\ln |x|+x<\frac{x}{2} \leq \ln \left(\frac{\delta}{(1-\mu)|\lambda|}\right)
$$

which also yields the result. In particular, the function $h_{\delta, \mu}(x)$ equals zero at $x=2 \ln \left(\frac{\delta}{(1-\mu)|\lambda|}\right)$.

Proposition 2.29. For every $\lambda \in \mathbb{C}^{*}, \delta \in(0,1)$ and $x<\sigma_{\mu}, h_{\delta, \mu}(x)$ is monotonically decreasing.

Proof. For simplicity in this proof we use the notation $A:=\frac{\delta}{(1-\mu)|\lambda|}$. Notice that $A$ is always positive. Then rewriting $h_{\delta, \mu}(x)=\sqrt{A^{2} e^{-2 x}-x^{2}}$ we have

$$
h_{\delta, \mu}^{\prime}(x)=\frac{-2 A^{2} e^{-2 x}-2 x}{2 h_{\delta, \mu}(x)}=\frac{-A^{2} e^{-2 x}-x}{\sqrt{A^{2} e^{-2 x}-x^{2}}} .
$$

To show that $h_{\delta, \mu}(x)$ is monotonically decreasing, we show $-A^{2} e^{-2 x}-x$ is negative. Since $x \leq \sigma_{\mu}<0$, we write $s=-x>0$ and distinguished between the following two cases.

Case 1: $x<-e<2 \ln A$. Then $s>e$ and we have $0<3-e=3 \ln e-e<3 \ln s-e$ as well as $\ln s<4 \ln s+2 \ln A<2 s+2 \ln A$. The latter inequality is equivalent to $s<A^{2} e^{2 s}$ which implies in turn $-A^{2} e^{-2 x}-x<0$.

Case 2: $x<2 \ln A<-e$. Then we obtain for positive values $-s<2 \ln A$, implying that $\ln s<s=2 s-s<2 s+2 \ln A$. Again, the latter inequality is equivalent to $s<A^{2} e^{2 s}$ providing the same result as in the first case.

We remark that the boundary of $\mathcal{M}_{\delta, \mu}$ is symmetric with respect to the real axis, as the domain $\mathcal{M}_{c, \mu}$ does. Therefore $\partial \mathcal{M}_{\delta, \mu}$ is monotonically decreasing for values above the real axis and monotonically increasing for values above the real axis. The previous proposition implies in particular for values of $x \leq \sigma_{\mu}$ that $\partial \mathcal{M}_{\delta, \mu}$ is an unbounded simple curve.

\subsubsection{Invariance of domain $\mathcal{H}_{\eta, \rho}$ under $g_{\lambda, \mu}$}

We devote this subsection to invariance of the smaller domain $\mathcal{H}_{\eta, \rho}:=\mathcal{H}_{\eta} \cup \mathcal{M}_{\rho}$ contained in $\mathcal{M}_{\delta, \mu}$. It can be the case that $\mathcal{M}_{\delta, \mu}$ is mapped completely into $\mathcal{H}_{\eta, \rho}$, after several iterations, or even that $\mathcal{M}_{\delta, \mu}$ intersects the Julia set, in which 
case is the reasons not to be invariant. The occurrence of one behaviour or the other depends on the given parameter $\lambda$ and its relations with $\mu$ and $\delta$, as we show subsequently. In other words, there may exist $z \in \mathcal{M}_{\delta, \mu} \backslash \mathcal{H}_{\eta, \rho}$ belonging to the Julia set. Therefore we restrict to the smaller region $\mathcal{M}_{\rho}$ and construct an unbounded domain $\mathcal{H}_{\eta}$ contained in a half plane located at the left side of $\mathcal{M}_{\rho}$ where we can guarantee the invariance under the function $g_{\lambda, \mu}$.

Let $\mathcal{M}_{\delta, \mu}$ be the domain defined in the previous subsection by the expression (2.18) and bounded by the curves $\pm h_{\delta, \mu}(x)$ defined in Equation 2.20). Consider a fixed parameter $\delta=\frac{1}{2}$ and write the discriminant of $h_{1 / 2, \mu}$ as

$$
\rho_{\mu}(x)=\left(\frac{e^{-x}}{2(1-\mu)|\lambda|}\right)^{2}-x^{2} .
$$

We denote by

$$
\mathcal{M}_{\rho}:=\left\{z=x+i y: 1-\frac{1}{2 \mu}<x<x_{0} \quad \text { and } \quad y^{2}<\rho_{\mu}(x)\right\}
$$

the restriction of $\mathcal{M}_{\delta, \mu}$ to values of $x$ in the interval $\left(1-\frac{1}{2 \mu}, x_{0}\right)$, where $x_{0}$ satisfies $\rho_{\mu}(x) \geq 0$ for all $x \leq x_{0}$. In Lemma 2.28 we stated that the function $\rho_{\mu}(x)$ is not negative for $x \leq \sigma_{\mu}:=\min \left\{-e, 2 \ln \left(\frac{1}{2(1-\mu)|\lambda|}\right)\right\}$. In the following we refer to $\sigma_{\mu}$ simply as the value $x_{0}$ such that $\rho_{\mu}(x) \geq 0$ for every $x \leq x_{0}$.

For values of $x \leq 1-\frac{1}{2 \mu}$ we construct the domain $\mathcal{H}_{\eta}$ as follows. Let $\pm \eta_{\mu}(x)$ be straight lines that join the origin with the points $z_{\rho, \pm}=\left(1-\frac{1}{2 \mu}, \pm \sqrt{\rho_{\mu}\left(1-\frac{1}{2 \mu}\right)}\right)$. Notice that the points $z_{\rho, \pm}$ lie on the boundary of $\mathcal{M}_{\rho}$. We define the domain as

$$
\begin{array}{r}
\mathcal{H}_{\eta}:=\left\{z=x+i y: x \leq 1-\frac{1}{2 \mu} \text { and }|y|<\eta_{\mu}(x)\right\} \\
\text { with } \eta_{\mu}(x):=-x \sqrt{\left(\frac{\mu e^{(1-2 \mu) / 2 \mu}}{(2 \mu-1)(1-\mu)|\lambda|}\right)^{2}-1 .}
\end{array}
$$

Recalling Corollary 2.2 there exists a $\mu_{1}$ for every $\lambda \in \mathbb{C}^{*}$ such that $|\lambda|<e^{\frac{1}{\mu}-2} \frac{\mu^{2}}{1-\mu}$ for all $\mu \leq \mu_{1}<\frac{1}{2}$. Under this assumption the existence of the attracting fixed point $z_{\mu}$ is guaranteed. However, we show below in Lemma 2.32 that we require a smaller boundary value of $\mu$ such that the invariance of the absorbing domain is assured for any given $\lambda$. In order to state the theorem on invariance we give here the necessary conditions on the parameters and remark that for these $\mu$ 's 


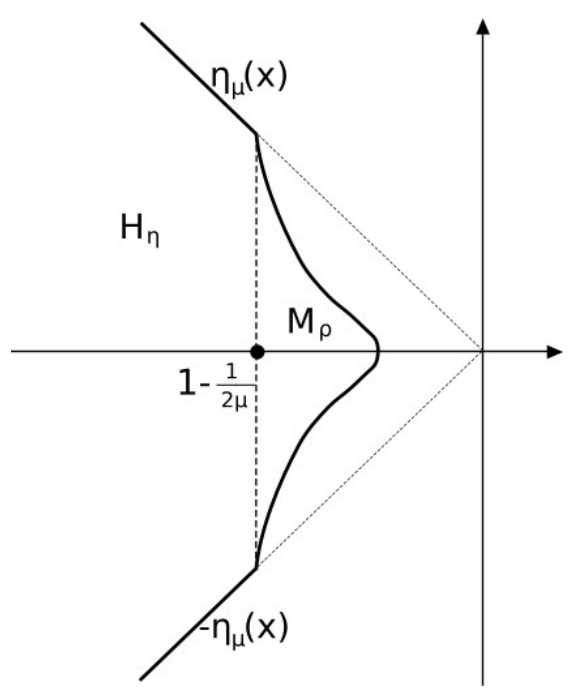

Figure 2.9: Invariant absorbing domain $\mathcal{H}_{\eta, \rho}$ defined as the union of two regions: firstly $\mathcal{H}_{\eta}$, which lies between the lines $\eta_{\mu}$ and $-\eta_{\mu}$, and secondly $\mathcal{M}_{\rho}$, which is a restriction of $\mathcal{M}_{1 / 2, \mu}$ for $\operatorname{Re} z>1-\frac{1}{2 \mu}$.

the existence of the attracting fixed point is automatically fulfilled. Let $\mu_{2}$ be sufficiently small that

$$
|\lambda|<e^{\frac{1}{2}\left(\frac{1}{\mu}-2\right)} \frac{\mu^{2}}{1-\mu} \quad \text { for all } \mu \leq \mu_{2}
$$

Now we obtain for the domains and the parameters as defined above the following result.

Theorem 2.30 (Invariance of absorbing domain). Let $\mathcal{H}_{\eta, \rho}:=\mathcal{H}_{\eta} \cup \mathcal{M}_{\rho}$. Then the domain $\mathcal{H}_{\eta, \rho}$ is invariant under $g_{\lambda, \mu}$ for every $\lambda \in \mathbb{C}^{*}$ and for every $\mu \in\left(0, \mu_{2}\right]$, with $\mu_{2}=\mu_{2}(\lambda)$.

We first present some lemmas needed for the proof. The proof of Theorem 2.30 follows in conclusion. A first tool to prove the invariance of the absorbing domain $\mathcal{H}_{\eta, \rho}$ is achieved by showing that two subdomains of $\overline{\mathcal{H}_{\eta, \rho}}$ are mapped into $\mathcal{H}_{\eta, \rho}$. The first region is given by the intersection $\overline{\mathcal{M}_{\delta, \mu}}$ with a right half-plane and we prove that it is mapped to the left. The second region is the intersection of $\overline{\mathcal{H}_{\eta, \rho}}$ with an upper and a lower half-planes which are mapped towards the real axis. 
Let $\delta \in(0,1)$. We define

$$
\begin{aligned}
\mathcal{H}_{R e} & :=\mathcal{M}_{\delta, \mu} \cap\left\{z \in \mathbb{C}: \operatorname{Re} z>1-\frac{1}{\mu}+\frac{\delta}{\mu}\right\} \text { and } \\
\mathcal{H}_{\text {Im }} & :=\mathcal{M}_{\delta, \mu} \cap\left\{z \in \mathbb{C}:|\operatorname{Im} z|>\frac{\delta}{\mu}\right\} .
\end{aligned}
$$

Lemma 2.31. For the domains defined above we obtain

- If $z \in \overline{\mathcal{H}_{R e}}$, then $\operatorname{Re} g_{\lambda, \mu}(z)<\operatorname{Re} z$.

- If $z \in \overline{\mathcal{H}_{\text {Im }}}$, then $\left|\operatorname{Im} g_{\lambda, \mu}(z)\right|<|\operatorname{Im} z|$.

Proof. Firstly consider $z \in \overline{\mathcal{H}_{R e}}$. In particular $z$ is also contained in $\overline{\mathcal{M}_{\delta, \mu}}$, therefore $(1-\mu)\left|\lambda z e^{z}\right| \leq \delta$ is satisfied. On the one hand we have $z \in \overline{\mathcal{H}_{R e}}$ if and only if $-\mu \operatorname{Re} z \leq 1-\mu-\delta$ which is equivalent to

$$
(1-\mu)(\operatorname{Re} z-1)+\delta \leq \operatorname{Re} z
$$

On the other hand, $\operatorname{Re} g_{\lambda, \mu}(z) \leq(1-\mu) \operatorname{Re}(z-1)+(1-\mu)\left|\lambda z e^{z}\right|$ holds. Since $z \in \overline{\mathcal{M}_{\delta, \mu}}$ this implies

$$
\operatorname{Re} g_{\lambda, \mu}(z) \leq(1-\mu)(\operatorname{Re} z-1)+\delta .
$$

In the first case equality holds for $z$ with $\operatorname{Re} z=1-\frac{1}{\mu}+\frac{\delta}{\mu}$, that is, on the left side of $\mathcal{H}_{R e}$. In the second case we obtain equality only for $z \in \mathbb{R} \cap \partial \mathcal{M}_{\delta, \mu}$, that is, on the right side of $\mathcal{H}_{R e}$. We can resume that both equalities never occur simultaneously for $z \in \mathbb{C}$. This assures the strict inequality $\operatorname{Re} g_{\lambda, \mu}(z)<\operatorname{Re} z$.

Secondly let $z \in \overline{\mathcal{H}_{I m}}$. We prove the result for $\operatorname{Im} z \geq \frac{\delta}{\mu}$ and it analogously follows for $\operatorname{Im} z \leq-\frac{\delta}{\mu}$. The inequality $\operatorname{Im} z \geq \frac{\delta}{\mu}$ is equivalent to $(1-\mu) \operatorname{Im} z+\delta \leq \operatorname{Im} z$. Then such a point also satisfies $\operatorname{Im} g_{\lambda, \mu}(z) \leq(1-\mu) \operatorname{Im} z+(1-\mu)\left|\lambda z e^{z}\right|$. This yields that

$$
\operatorname{Im} g_{\lambda, \mu}(z) \leq(1-\mu) \operatorname{Im} z+\delta
$$

In this case equality holds if the intersection of $\partial \mathcal{H}_{I m}$ with $\partial \mathcal{M}_{\delta, \mu}$ lies on $i \mathbb{R}$. However it is not possible, because $\operatorname{Re} z<0$ for all $z \in \partial \mathcal{M}_{\delta, \mu}$. Hence we obtain a strict inequality as well.

In order to proof that the domain $H_{\eta, \rho}$ is invariant with respect to $g_{\lambda, \mu}$, we firstly show that $g_{\lambda, \mu}\left(\partial H_{\eta, \rho}\right) \subset H_{\eta, \rho}$. The latter statement follows almost immediately from the lemma above if $\mathcal{H}_{R e} \cap \mathcal{H}_{I m} \neq \emptyset$. A non-empty intersection is assured 

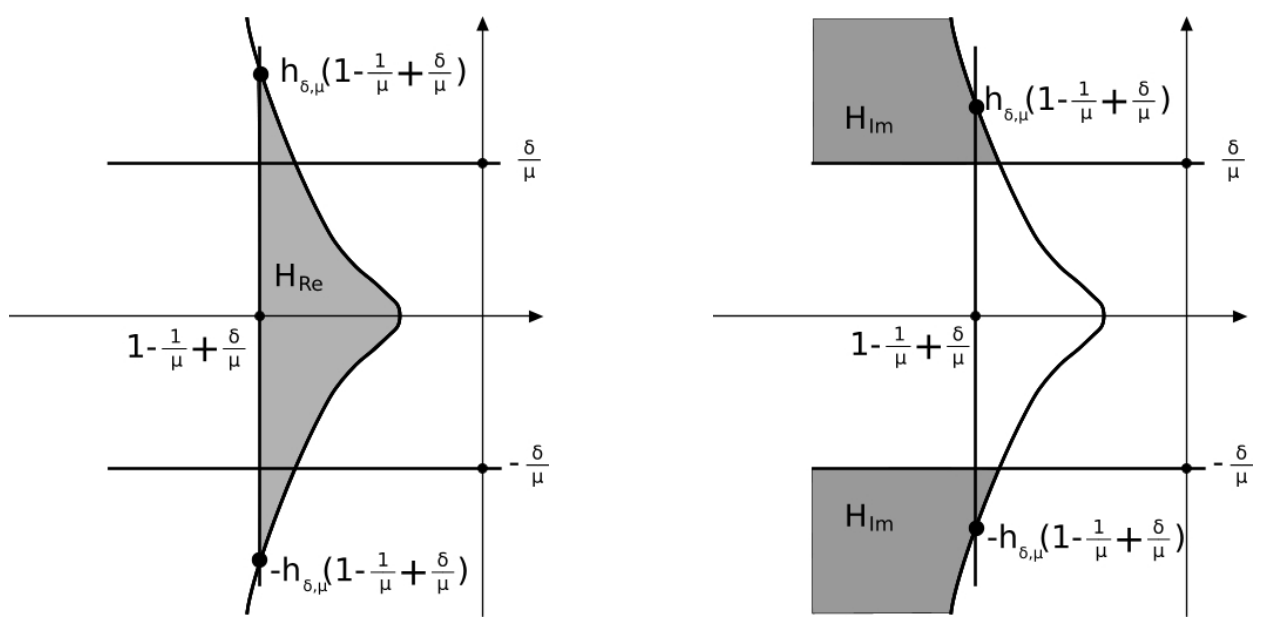

Figure 2.10: Domain $\mathcal{M}_{\delta, \mu}$ together with regions $\mathcal{H}_{I m}$ and $\mathcal{H}_{R e}$, where $\left|\operatorname{Im} g_{\lambda, \mu}(z)\right|<|\operatorname{Im} z|$ and $\operatorname{Re} g_{\lambda, \mu}(z)<\operatorname{Re} z$ respectively. The intersection of $\mathcal{H}_{R e}$ and $\mathcal{H}_{\text {Im }}$ is non empty for $\delta \leq \frac{1}{2}$.

when $h_{\delta, \mu}\left(1-\frac{1}{\mu}+\frac{\delta}{\mu}\right)>\frac{\delta}{\mu}$. For $\delta=1$ the inequality reduces to $h_{\delta, \mu}(1)>\frac{1}{\mu}$ which is fulfilled if and only if $e^{-2}>|\lambda|^{2}(1-\mu)^{2}\left(1+\frac{1}{\mu^{2}}\right)$. This is a strong restriction, hardly satisfied by an arbitrary parameter $\lambda$. In other words, we can always find values of $\lambda$ such that the intersection is empty, even when we take the limit $\mu \rightarrow 0$.

In contrast to this, consider now a small value of $\delta \in(0,1)$, for example $\delta=\mu$. Then the intersection of $\mathcal{H}_{R e}$ and $\mathcal{H}_{I m}$ is non empty, since it is equivalent to $2 \sqrt{\frac{1}{\mu}-1}>1$. But with this, the disc $D((1-\mu)(z-1), \delta)$ containing $g_{\lambda, \mu}(z)$ as defined for points $z \in \mathcal{M}_{\delta, \mu}$ collapses to a point as we take the limit $\delta=\mu \rightarrow 0$.

Following these arguments, we may restrict the value of $\delta$ so that we can continue with the analysis for arbitrary values of $\lambda \in \mathbb{C}^{*}$ and $\mu \in\left(0, \mu_{2}\right]$. Let $\delta \leq \frac{1}{2}$ and $\mu_{2}$ be sufficiently small that Equation (2.24) is fulfilled for all $\mu \leq \mu_{2}$. Then we have as well that $\frac{1}{|\lambda|^{2}}>e^{\left(2-\frac{1}{\mu}\right)} \frac{(1-\mu)^{2}}{\mu^{4}}$.

We claim that $\mathcal{H}_{R e}$ is not empty for $\delta=\frac{1}{2}$. We prove it by showing that $\rho_{\mu}\left(1-\frac{1}{\mu}+\frac{\delta}{\mu}\right)>0$ for values of $\mu_{2}$ and $\lambda$ as above. After some computation, 
we obtain

$$
\begin{aligned}
\rho_{\mu}\left(1-\frac{1}{\mu}+\frac{\delta}{\mu}\right) & =\left(\frac{\delta}{1-\mu}\right)^{2} \frac{1}{|\lambda|^{2}} e^{-2\left(1-\frac{1}{\mu}+\frac{\delta}{\mu}\right)}-\left(1-\frac{1}{\mu}+\frac{\delta}{\mu}\right)^{2} \\
& >\frac{\delta^{2}}{\mu^{4}} e^{2-\frac{1}{\mu}} e^{-2\left(1-\frac{1}{\mu}+\frac{\delta}{\mu}\right)}-\left(1-\frac{1}{\mu}+\frac{\delta}{\mu}\right)^{2}
\end{aligned}
$$

which is positive if and only if $e^{\frac{1}{\mu}(1-2 \delta)}>\frac{\mu^{2}}{\delta^{2}}(\mu-1+\delta)^{2}$. Then for $\delta=\frac{1}{2}$ we obtain $e^{\frac{1}{\mu}(1-2 \delta)}=1>4 \mu^{2}\left(\mu-\frac{1}{2}\right)$ for all $0<\mu \leq \frac{1}{2}$.

In what follows, we consider the fixed value $\delta=\frac{1}{2}$ and denote the function $h_{\delta, \mu}$ defined in Equation 2.20 simply by $h_{\mu}$. Recall that $h_{\mu}(x)=\sqrt{\rho(x)}$ as described in Equation (2.21).

Lemma 2.32. Let $\delta=\frac{1}{2}$ and $\mu_{2}$ sufficiently small that $|\lambda|<e^{\frac{1}{2}\left(\frac{1}{\mu}-2\right)} \frac{\mu^{2}}{1-\mu}$ for all $\mu \leq \mu_{2}$. Then $\mathcal{H}_{R e} \cap \mathcal{H}_{\text {Im }} \neq \emptyset$.

Proof. We show that $h_{\mu}\left(1-\frac{1}{2 \mu}\right)>\frac{1}{2 \mu}$. On the one hand

$$
\begin{aligned}
h_{\mu}\left(1-\frac{1}{2 \mu}\right) & =\sqrt{\left(\frac{1}{2(1-\mu)|\lambda|}\right)^{2} e^{-2\left(1-\frac{1}{2 \mu}\right)}-\left(1-\frac{1}{2 \mu}\right)^{2}} \\
& >\sqrt{\frac{1}{4(1-\mu)^{2}} e^{-\left(\frac{1}{\mu}-2\right)} \frac{(1-\mu)^{2}}{\mu^{4}} e^{\frac{1}{\mu}-2}-\left(1-\frac{1}{2 \mu}\right)^{2}} \\
& =\sqrt{\frac{1}{4 \mu^{4}}-\left(1-\frac{1}{2 \mu}\right)^{2}}
\end{aligned}
$$

On the other hand, the latter expression is larger than $\frac{1}{2 \mu}$ if and only if

$$
\frac{1}{4 \mu^{4}}>\left(1-\frac{1}{2 \mu}\right)^{2}+\frac{1}{4 \mu^{2}}=\frac{(2 \mu-1)^{2}+1}{4 \mu^{2}} .
$$

The inequality above is equivalent to $1>\mu^{2}(2 \mu-1)^{2}+\mu^{2}$. Finally, for all $\mu<\left(0, \frac{1}{2}\right]$ it follows that $(2 \mu-1)^{2}$ lies in the interval $[0,1)$ and with this $\mu^{2}\left((2 \mu-1)^{2}+1\right)<\frac{1}{2}$, yielding the desired result.

An important consequence of choosing the parameter $\delta=\frac{1}{2}$ is stated as follows.

Corollary 2.33. For $\delta=\frac{1}{2}$ the domains $\mathcal{H}_{\text {Re }}$ and $\mathcal{M}_{\rho}$ coincide. 
A second tool in the proof of the invariance of $\mathcal{H}_{\eta, \rho}$ is to characterise points $z \in \mathcal{M}_{\delta, \mu}$ that are at a distance $\frac{1}{2}$ from the point $(1-\mu)(z-1)$. We prove in the following lemma that a point $z$ belongs to such a disc if and only if $z$ lies in a neighbourhood of the attracting fixed point $z_{\mu}$ tangent to $\mathcal{H}_{R e}$ and $\mathcal{H}_{I m}$ as it is shown in Figure 2.11. Recall that for $\delta=1 / 2$ these domains simplify by

$$
\begin{aligned}
& \mathcal{H}_{R e} \subset\left\{z \in \mathbb{C}: \operatorname{Re} z>1-\frac{1}{2 \mu}\right\} \quad \text { and } \\
& \mathcal{H}_{\text {Im }} \subset\left\{z \in \mathbb{C}:|\operatorname{Im} z|>\frac{1}{2 \mu}\right\} .
\end{aligned}
$$

Corollary 2.34. Let $\delta=1 / 2$ and $z \in \mathcal{M}_{1 / 2, \mu}$. Then the point $z$ is contained in $D\left((1-\mu)(z-1), \frac{1}{2}\right)$ if and only if $z \in D\left(1-\frac{1}{\mu}, \frac{1}{2 \mu}\right)$.

Proof. Let $z \in \mathcal{M}_{1 / 2, \mu}$. Using definition the definition of $\mathcal{M}_{1 / 2, \mu}$ we obtain that $z$ lie in the $\operatorname{disc} D\left(1-\frac{1}{\mu}, \frac{1}{2 \mu}\right)$, that is

$$
\left|z-\left(1-\frac{1}{\mu}\right)\right|=\left|z+\left(\frac{1-\mu}{\mu}\right)\right|<\frac{1}{2 \mu}
$$

which is equivalent to $|\mu z+1-\mu|=|z-(1-\mu)(z-1)|<\frac{1}{2}$. It means, $z$ lies as well in the disc $D\left((1-\mu)(z-1), \frac{1}{2}\right)$.

For the rest of the section we assume $\delta=\frac{1}{2}$ and $\frac{1}{|\lambda|^{2}}>e^{-\left(\frac{1}{\mu}-2\right) \frac{(1-\mu)^{2}}{\mu^{4}}}$ for all $\mu \leq \mu_{2}$, as stated in Theorem 2.30. Furthermore, we show the results for points $z$ with $\operatorname{Im} z>0$ and they analogously apply for $z$ with $\operatorname{Im} z<0$, following the same argument for the functions $-\eta_{\mu}(x)$ and $-h_{\mu}(x)$.

Proof of Theorem 2.30. Now we prove the invariance of the domain $\mathcal{H}_{\eta, \rho}$. It is clear that the attracting fixed point $z_{\mu}$ is contained in $\mathcal{H}_{\eta, \rho}$, since $z_{\mu}$ lies in the $\operatorname{disc} D\left(1-\frac{1}{\mu}, 1\right) \subset D\left(1-\frac{1}{\mu}, \frac{1}{2 \mu}\right)$ for all $\mu<\frac{1}{2}$. Then it is enough to show that $g_{\lambda, \mu}\left(\partial \mathcal{H}_{\eta, \rho}\right) \subset \mathcal{H}_{\eta, \rho}$.

Let $z_{b}$ be an arbitrary point in the boundary of $\mathcal{H}_{\eta, \rho}$ and denote the disc containing its image $g_{\lambda, \mu}\left(z_{b}\right)$ by $D_{b}:=D\left((1-\mu)\left(z_{b}-1\right), \frac{1}{2}\right)$. Notice that $z_{b} \in \overline{\mathcal{H}_{R e}} \cup \overline{\mathcal{H}_{I m}}$. Hence using Corollary 2.34, $z_{b} \notin D_{b}$. We show either directly $g_{\lambda, \mu}\left(z_{b}\right) \in \mathcal{H}_{\eta, \rho}$ or that the whole disc $D_{b}$ is contained in $\mathcal{H}_{\eta, \rho}$.

Firstly, assume $z_{b} \in \overline{\mathcal{M}_{\rho}} \cap \partial \mathcal{H}_{\eta, \rho}$ as it can be seen in Figure 2.9. Since $\mathcal{H}_{R e}$ and $\mathcal{M}_{\rho}$ coincide for $\delta=\frac{1}{2}$, we obtain $\operatorname{Re} g_{\lambda, \mu}\left(z_{b}\right)<\operatorname{Re} z_{b}$. Since the curve 


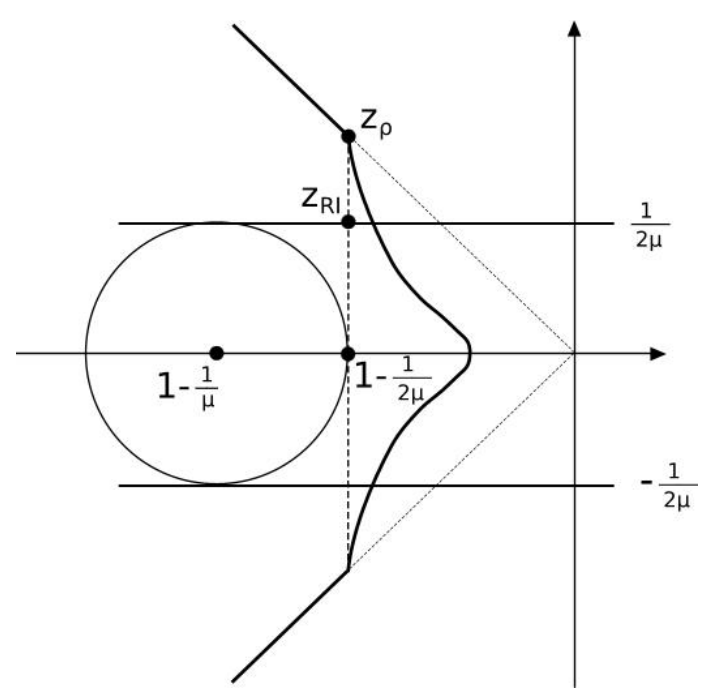

Figure 2.11: Invariant fundamental domain $\mathcal{H}_{\eta, \rho}$ defined for $\delta=$ $1 / 2, \mu \leq \mu_{2}$. The disc $D\left(1-\frac{1}{\mu}, \frac{1}{2 \mu}\right)$ is tangent to the boundaries of the domains $\mathcal{H}_{\text {Re }}$ and $\mathcal{H}_{\text {Im }}$.

$\partial \mathcal{M}_{\rho}$ is monotonically decreasing (reps. monotonically increasing) for point with imaginary part greater (resp. lower) than zero as proved in Proposition 2.29, we have that $g_{\lambda, \mu}\left(z_{b}\right) \in \mathcal{M}_{\rho}$. In particular this implies that the whole disc $D_{b}$ is as well contained in $\mathcal{M}_{\rho}$.

Secondly, write $z_{\rho}:=\left(1-\frac{1}{2 \mu}, \sqrt{\rho_{\mu}\left(1-\frac{1}{2 \mu}\right)}\right)$ for the boundary point lying on $\partial \mathcal{M}_{\rho}$ such that $\operatorname{Re} z_{\rho}<\operatorname{Re} z$ for every $z \in \mathcal{M}_{\rho}$ as it is marked in Figure 2.11. Using Lemma $2.32 z_{\rho} \in \mathcal{H}_{R e} \cap \mathcal{H}_{I m}$. Hence $\operatorname{Re} g_{\lambda, \mu}\left(z_{\rho}\right)<\operatorname{Re} z_{\rho}$ and $\operatorname{Im} g_{\lambda, \mu}\left(z_{\rho}\right)<\operatorname{Im} z_{\rho}$ which implies that $g_{\lambda, \mu}\left(z_{\rho}\right) \in \mathcal{H}_{\eta, \rho}$.

Thirdly, consider a point $z_{b} \in \partial \mathcal{H}_{\eta}$. Then it satisfies $z_{b}=x_{b}+i \eta_{\mu}\left(x_{b}\right)$ and we refer the reader to Figure 2.12 for a sketch of the following computations. Recall that every $z_{b} \in \partial \mathcal{H}_{\eta} \subset \mathcal{M}_{1 / 2, \mu}$ satisfies $(1-\mu)\left|\lambda z_{b} e^{z_{b}}\right|<\frac{1}{2}$. Hence we obtain

$$
\begin{aligned}
\operatorname{Re} g_{\lambda, \mu}\left(z_{b}\right) & =(1-\mu)\left(x_{b}-1\right)+(1-\mu) \operatorname{Re}\left(\lambda z_{b} e^{z_{b}}\right) \\
& \leq(1-\mu)\left(x_{b}-1\right)+(1-\mu)\left|\lambda z_{b} e^{z_{b}}\right|<(1-\mu)\left(x_{b}-1\right)+\frac{1}{2}
\end{aligned}
$$

Analogously $\operatorname{Im} g_{\lambda, \mu}\left(z_{b}\right)<(1-\mu) \operatorname{Im} z_{b}+\frac{1}{2}$ holds. We prove that $g_{\lambda, \mu}\left(z_{b}\right)$ lies in $\mathcal{H}_{\eta}$ by showing that $\operatorname{Im} g_{\lambda, \mu}\left(z_{b}\right)<\eta_{\mu}\left(\operatorname{Re} g_{\lambda, \mu}\left(z_{b}\right)\right)$. Since $z_{b} \in \partial \mathcal{H}_{\eta}$, it suffices to 
show that

$$
(1-\mu) \eta_{\mu}\left(x_{b}\right)+\frac{1}{2}<\eta_{\mu}\left((1-\mu)\left(x_{b}-1\right)+\frac{1}{2}\right) .
$$

Since $\eta_{\mu}(x)$ is a linear function, we rewrite the previous inequality as $\frac{1}{2}<\eta_{\mu}\left(\mu-\frac{1}{2}\right)$. If we compute directly

$$
\eta_{\mu}\left(\mu-\frac{1}{2}\right)=\left(\frac{1}{2}-\mu\right) \sqrt{\left(\frac{\mu}{(2 \mu-1)(1-\mu)}\right)^{2} \frac{1}{|\lambda|^{2}} e^{\frac{1}{\mu}-2}-1}
$$

we obtain the inequality

$$
\frac{1}{(2-\mu)^{2}}<\left(\frac{\mu}{(2 \mu-1)(1-\mu)}\right)^{2} \frac{1}{|\lambda|^{2}} e^{\frac{1}{\mu}-2}-1
$$

Recall that the parameter $\lambda$ satisfies $\frac{1}{|\lambda|^{2}}>e^{\left(2-\frac{1}{\mu}\right) \frac{(1-\mu)^{2}}{\mu^{4}}}$. Then the right side of the latter inequality is greater than

$$
\left(\frac{\mu}{(2 \mu-1)(1-\mu)}\right)^{2} e^{-\left(\frac{1}{\mu}-2\right)} \frac{(1-\mu)^{2}}{\mu^{4}} e^{\frac{1}{\mu}-2}-1=\frac{1}{(2 \mu-1) \mu^{2}}-1 .
$$

Finally comparing the latter two computations, the Inequality (2.25) is satisfied provided $1+(2 \mu-1)^{2}<\frac{1}{\mu^{2}}$ which is valid for every $\mu<\frac{1}{2}$.

Fourthly, we show for completeness that the point $z_{R I}=1-\frac{1}{2 \mu}+i \frac{1}{2 \mu}$, lying at the left bottom of $\overline{\mathcal{H}_{R e}} \cap \overline{\mathcal{H}_{\text {Im }}}$ as marked in Figure 2.11, is also mapped inside. In particular, it holds

$$
\operatorname{Re} g_{\lambda, \mu}\left(z_{R I}\right)<(1-\mu)\left(1-\frac{1}{2 \mu}-1\right)+\frac{1}{2}=1-\frac{1}{2 \mu}=\operatorname{Re} z_{R I}
$$

and

$$
\operatorname{Im} g_{\lambda, \mu}\left(z_{R I}\right)<(1-\mu)\left(\frac{1}{2 \mu}\right)+\frac{1}{2}=\frac{1}{2 \mu}=\operatorname{Im} z_{R I}
$$

The arguments presented during the whole proof are analogously satisfied for points of $\mathcal{H}_{\eta, \rho}$ with negative imaginary part. Due to continuity, the intersection of the real axis with $\mathcal{H}_{\eta, \rho}$ is also mapped inside of $\mathcal{H}_{\eta, \rho}$ by $g_{\lambda, \mu}$, completing the proof

We have shown in Theorem 2.30, that the domain $\mathcal{H}_{\eta, \rho}$ is forward invariant. Now we prove that it is an absorbing domain for $\mathcal{A}_{\mu}$. For this it is left to show that given any compact set $K$ in the basin of attraction $\mathcal{A}_{\mu}$, there exists an $n \in \mathbb{N}$ such that $g_{\lambda, \mu}^{n}(K) \subset \mathcal{H}_{\eta, \rho}$. We show it in the following result. 


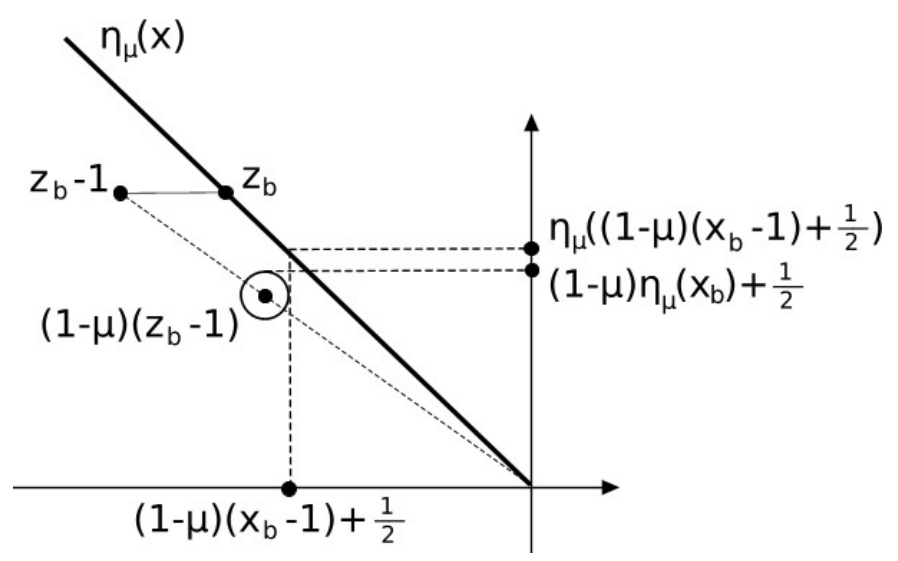

Figure 2.12: For a boundary point $z_{b}=x_{b}+i \eta_{\mu}\left(x_{b}\right)$ on $\partial \mathcal{H}_{\eta}$ the disc containing its image $g_{\lambda, \mu}\left(z_{b}\right)$ denoted by $D_{b}:=D\left((1-\mu)\left(z_{b}-1\right), \frac{1}{2}\right)$ is mapped inside of $\mathcal{H}_{\eta, \rho}$.

Proposition 2.35. The domain $\mathcal{H}_{\eta, \rho} \subset \mathcal{A}_{\mu}$ is absorbing for $g_{\lambda, \mu}$.

Proof. Let $K$ be any compact set contained in the basin of attraction $\mathcal{A}_{\mu}$. Let $D_{\mu}$ denote the disc $D\left(\left(1-\frac{1}{\mu}\right), \frac{1}{2 \mu}\right) \subset \mathcal{A}_{\mu}$ and recall that $D_{\mu} \subset \mathcal{H}_{\eta, \rho}$ is tangent to $\mathcal{H}_{R e}$ and $\mathcal{H}_{I m}$. In particular $D_{\mu}$ contains the attracting fixed point $z_{\mu}$ and all points $z$ in $D_{\mu}$ convergence to $z_{\mu}$ under iterates. Even more, for every $z \in \mathcal{A}_{\mu}$ exists an $n \in \mathbb{N}$ such that $g_{\lambda, \mu}^{n}(z) \in D_{\mu}$. Hence, $\mathcal{A}_{\mu} \subset \bigcup_{n \in \mathbb{N}} g_{\lambda, \mu}^{-n}\left(D_{\mu}\right)$. In particular, this covers the compact set $K$. Then there exists a finite subcovering $\bigcup_{m \in I} g_{\lambda, \mu}^{-m}\left(D_{\mu}\right)$ of $K$ with a finite index set $I \subset\left\{0, \ldots, n_{0}\right\}$. This implies that $g_{\lambda, \mu}^{n_{0}}(K) \subset D_{\mu}$ and in particular it is also contained in $\mathcal{H}_{\eta, \rho}$ for some $n \leq n_{0}$.

It was proved by Lauber in [48] that $\mathcal{M}_{\delta}$ contains all critical values, with at most one exception. In comparison to this and together with the properties of critical points and values presented in the previous section we show that a finite number of the critical values of $g_{\lambda, \mu}$ are also contained in $\mathcal{H}_{\eta, \rho}$. More precisely we obtain the following result.

Corollary 2.36. A finite number of critical values $v_{k}$ are contained in the $a b$ sorbing domain $\mathcal{H}_{\eta, \rho}$ while all critical points $c_{k}$ lie outside of it.

Proof. As we showed at the beginning of this section in Equation (2.19), the boundary of $\mathcal{M}_{\frac{1}{2}, \mu}$ is approximately a translation by one to the left of $\partial \mathcal{M}_{c, \mu}$ where the critical points are located, yielding the second statement. Since $v_{k} \in$ 
$D\left(c_{k}-2, \frac{1}{2}\right)$ using Lemma 2.25 then that all critical values are in $\mathcal{M}_{\frac{1}{2}, \mu}$ by definition of the domain. In other words, the critical values are in the preliminary domain $\mathcal{M}_{\frac{1}{2}, \mu}$, before we take the restriction to the smaller domain $\mathcal{M}_{\rho}$. With this, we obtain only a finite number of them in $\mathcal{H}_{\eta, \rho}$, that is only the first $|k|$ contained in $\mathcal{M}_{\rho}$.

In other words, Corollary 2.36 implies that $g_{\lambda, \mu}$ is univalent in the absorbing domain. However, since the Baker domain is not univalent, it follows that its boundary is not a Jordan curve, using a result due to Baker and Weinreich mentioned in the introduction. In the following sections we use the location of the critical points and values as stated in the corollary above to construct further structure in the basin of attraction outside the absorbing domain. This enable us a better understanding of the Julia set of $g_{\lambda, \mu}$.

\subsection{Further structures in $\mathcal{F}\left(g_{\lambda, \mu}\right)$}

We now present some results about the structure of the basin of attraction $\mathcal{A}_{\mu}$. In the previous section we constructed an invariant absorbing domain for $g_{\lambda, \mu}$, denoted by $\mathcal{H}_{\eta, \rho}$. In this section, we use some of the characteristics of the absorbing domain, as the position of the critical points and values with respect to its boundary $\partial \mathcal{H}_{\eta, \rho}$, in order to construct a family of invariant Jordan curves $\left\{\Gamma_{k, \mu}\right\}_{k}$. The curves $\Gamma_{k, \mu}$ describe the structure of the basin of attraction $\mathcal{A}_{\mu}$ beyond the absorbing domain and enable us to give an approximated position of the Julia set in strips bounded above and below by two subsequent curves. In Section 2.6, we use the Jordan curves $\left\{\Gamma_{k, \mu}\right\}_{k}$ to construct a domain $V$ depending on $\lambda$ and $\mu$ where the restriction of $g_{\lambda, \mu}$ forms a polynomial-like triple for every $\mu<\mu_{0}$.

Let $\lambda \in \mathbb{C}^{*}$ and $\mu_{0} \in\left(0, \frac{1}{2}\right)$ be such that the invariance of the absorbing domain is satisfied. From now on consider $\mu \leq \mu_{0}$. Furthermore, consider the critical values $v_{k}$ with $k \neq 0$ contained in the absorbing domain $\mathcal{H}_{\eta, \rho}$ as stated in Corollary 2.36. For each one of these values $v_{k}$ we define an invariant curve $\Gamma_{k, \mu}$ containing the corresponding critical point $c_{k}$ and joining the critical value with the attracting fixed point $z_{\mu}$ at one end of the curve. At the other end, the curve $\Gamma_{k, \mu}$ tends horizontal asymptotically to $\infty$ for points with large positive real part. The case for $k=0$ is not considered since the function $g_{\lambda, \mu}$ may have more than one critical point in a neighbourhood of $\mathbb{R}$ for arbitrary $\lambda$. In the following theorem we describe these curves in more detail. 
Theorem 2.37 (Structure Theorem). Let $c_{k}$ and $v_{k}$ be a critical point and its value for some $k \in \mathbb{Z}^{*}$. Then there exists an $N \in \mathbb{N}, N=N(\mu)$, and a family of Jordan curves $\left\{\Gamma_{k, \mu}\right\}_{k}$ with $|k| \leq N, k \neq 0$, and $\Gamma_{k, \mu}: t \mapsto \gamma_{k}(t)$ for $t \in[0, \infty)$. The family $\left\{\Gamma_{k, \mu}\right\}_{k}$ has the following properties

i) For each $k$, there is exactly one critical point $c_{k}$ on $\Gamma_{k, \mu}$.

ii) For each $\Gamma_{k, \mu}$ we have: $\gamma_{k}(0)=z_{\mu}$ and $\operatorname{Re}\left(\gamma_{k}(t)\right) \rightarrow \infty$ as $t \rightarrow \infty$ while $\operatorname{Im}\left(\gamma_{k}(t)\right)$ is bounded.

iii) The curves $\Gamma_{k, \mu} \backslash\left\{\gamma_{k}(0)\right\}$ are pairwise disjoint.

iv) For each $k$, the set $\Gamma_{k, \mu} \cup\left(-\infty, z_{\mu}\right)$ is completely invariant.

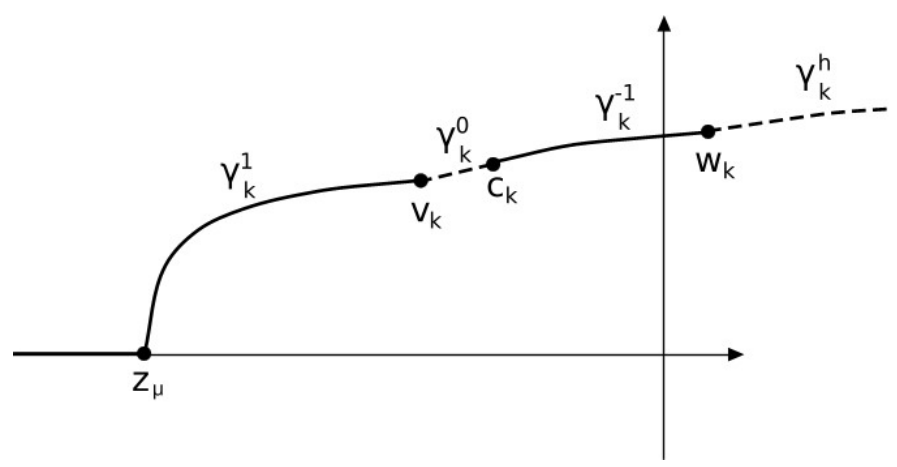

Figure 2.13: Sketch of curve $\Gamma_{k, \mu}$ constructed as the union $\gamma_{k}^{1} \cup \gamma_{k}^{0} \cup \gamma_{k}^{-1} \cup \gamma_{k}^{h}$ for some $k>0$.

Proof. We denote by $N=N(\mu)$, the maximal index such that the critical value $v_{k} \in \mathcal{M}_{\rho}$. Then it holds $\operatorname{Im} v_{k}<\sqrt{\rho_{\mu}\left(1-\frac{1}{2 \mu}\right)}$ for every $|k| \leq N$. For $k \neq 0$ let $c_{k}$ the critical point such that $g_{\lambda, \mu}\left(c_{k}\right)=v_{k}$. We describe the proof for $k>0$ and the case for $k<0$ follows analogously. Each curve $\Gamma_{k, \mu}$ will be constructed as the union of four simple arcs as follows:

i) The $\operatorname{arc} \gamma_{k}^{0}$ joins the critical point $c_{k}$ with its critical value $v_{k}$.

ii) The arc $\gamma_{k}^{1}$ continues from $\gamma_{k}^{0}$ and joins the critical value $v_{k}$ to the fixed point $z_{\mu}$.

iii) Write $w_{k}$ for the preimage of the fixed point $z_{\mu}$ under the inverse branch $h_{k}$ of $g_{\lambda, \mu}$ that satisfies $h\left(v_{k}\right)=c_{k}$. Then the arc $\gamma_{k}^{-1}$ goes from $c_{k}$ to $w_{k}$.

iv) Finally, the arc $\gamma_{k}^{h}$ is constructed as the preimage of the interval $\left(-\infty, z_{\mu}\right]$ 
under the same inverse branch $h_{k}$ of $g_{\lambda, \mu}$. Furthermore, $\gamma_{k}^{h}$ extends asymptotically horizontal from $w_{k}$ to $\infty$.

We prove the properties stated in the theorem by the time we describe the construction of the curves $\left\{\Gamma_{k, \mu}\right\}_{k}$.

Consider at first a simple arc joining $c_{k}$ with $v_{k}$ and denote it by $\gamma_{k}^{0}$. We define $\gamma_{k}^{1}:=\bigcup_{n=1}^{\infty} g_{\lambda, \mu}^{n}\left(\gamma_{k}^{0}\right) \cup\left\{z_{\mu}\right\}$. Since $v_{k}$ lies in $\mathcal{H}_{\eta, \rho}$, then $g_{\lambda, \mu}^{n}\left(v_{k}\right) \in \mathcal{H}_{\eta, \rho}$ for all $n \in \mathbb{N}$ and $\lim _{n \rightarrow \infty} g_{\lambda, \mu}^{n}\left(v_{k}\right)=z_{\mu}$. Hence $\gamma_{k}^{1}$ is a continuous path joining $v_{k}$ with $z_{\mu}$. In particular, $\gamma_{k}^{1}$ is a simple arc, since $g_{\lambda, \mu}$ is univalent in $\mathcal{H}_{\eta, \rho}$ using Corollary 2.36.

We claim that $\gamma_{k}^{1} \backslash\left\{z_{\mu}\right\}$ and $\gamma_{j}^{1} \backslash\left\{z_{\mu}\right\}$ are pairwise disjoint for any $k \neq j$. Since the Euclidean distance between two subsequent critical values $v_{k-1}$ and $v_{k}$ is positive, we can always choose the arcs such that $\gamma_{k}^{0} \cap \gamma_{k-1}^{0}=\emptyset$. Hence $g_{\lambda, \mu}^{n}\left(\gamma_{k}^{0}\right) \cap g_{\lambda, \mu}^{n}\left(\gamma_{k-1}^{0}\right)=\emptyset$ for every $n \geq 1$, again since $g_{\lambda, \mu}$ is univalent in $\mathcal{H}_{\eta, \rho}$. The latter argument holds in particular for two subsequent critical values with indices $k$ and $k-1$, but also in general for any pair of critical values $v_{k}$ and $v_{j}$ with $k \neq j$.

Denote by $h_{k}$ the inverse branch of $g_{\lambda, \mu}$ such that $h_{k}\left(v_{k}\right)=c_{k}$. The curve $\gamma_{k}^{0} \cup \gamma_{k}^{1}$ is forward invariant and goes from a critical point $c_{k}$ to the fixed point $z_{\mu}$. Recall that $c_{k}$ is a simple critical point for all $k \neq 0$. Therefore the preimage of $\gamma_{k}^{1} \cup \gamma_{k}^{0}$ is mapped two-to-one onto itself by $g_{\lambda, \mu}$ and can be seen as consisting of two components. The first component of the preimage must be the curve $\gamma_{k}^{1} \cup \gamma_{k}^{0}$ itself. The second component joins the critical point $c_{k}$ with $w_{k}$, where the latter denotes the preimage of the fixed point $z_{\mu}$ under the inverse branch $h_{k}$. For every $k$ we define $\gamma_{k}^{-1}$ as the arc joining $c_{k}$ with $w_{k}$ as defined above.

We now construct $\gamma_{k}^{h}$ as the preimage of the interval $\left(-\infty, z_{\mu}\right)$ under the appropriate inverse branch $h_{k}$. From Section 2.2, there are neither critical values nor fixed points in $\left(-\infty, z_{\mu}\right)$. This means, there exists only one curve as preimage with respect to each inverse branch $h_{k}$ which is attached to $w_{k}$. We claim that the curve $\gamma_{k}^{h}$ extends from $w_{k}$ to $+\infty$. Otherwise, if the curve $\gamma_{k}^{h}$ is bounded also to the right, then the function $g_{\lambda, \mu}$ must map a bounded curve onto the unbounded interval $\left(-\infty, z_{\mu}\right)$, which is a contradiction to the fact that $g_{\lambda, \mu}$ has no finite asymptotic values.

Finally, we prove that the curves $\gamma_{k}^{h}$ tends asymptotically horizontal to $\infty$. For this consider a strip of the form $L:=\left\{z: \operatorname{Re} z<z_{\mu}+\varepsilon,|\operatorname{Im} z|<\varepsilon\right\}$ for some $\varepsilon \in(0,1)$. In other words, $L$ is an open neighbourhood of $\left(-\infty, z_{\mu}\right)$. Using continuity, the preimage of $L$ is mapped around the curve $\gamma_{k}^{h}$ by the inverse branch $h_{k}$ and belongs to the Fatou set. If we extend $h_{k}(L):=L_{k}^{h}$ to the left and around the curve $\Gamma_{k}$, 
then at some point we reach the critical point $c_{k}$. Consider now two subsequent open strips $L_{k}^{h}$ and $L_{k-1}^{h}$ defined as the preimages of $L$ under $h_{k}$ and $h_{k-1}$. If we extend them until $c_{k}$ and $c_{k-1}$ belong respectively to each of them, then there must exist a repelling fixed point lying in the Julia set located in between. More over, there exists a whole curve belonging to $\mathcal{J}\left(g_{\lambda, \mu}\right)$ that extends as well to $+\infty$ and which serves as separation between two preimages on $L$ under two different inverse branches. Therefore the curves $\gamma_{k}^{h}$ extend asymptotically horizontal to $\infty$, that is, their imaginary part is bounded as the real part goes to $\infty$, concluding our result.

Corollary 2.38. The basin of attraction $\mathcal{A}_{\mu}$ contains countable many horizontal strips unbounded to the right and contained in a right half-plane.

Even though only a finite number of critical points are contained in $\mathcal{H}_{\eta, \rho}$, the basin of attraction $\mathcal{A}_{\mu}$ contains an infinite set of disjoint horizontal right half strips that extend to $\infty$. These strips are the preimages of an open neighbourhood of the interval $\left(-\infty, z_{\mu}\right)$ as it was described in the proof above by the strip $L$. There must be countably many of these strips, since each one of these must contain a preimage of $z_{\mu}$, say $w_{k}$, under an inverse branch of $g_{\lambda, \mu}$.

\subsection{Polynomial-like mapping}

The Jordan curves $\left\{\Gamma_{k, \mu}\right\}_{k}$ give a structure in the basin of attraction $\mathcal{A}_{\mu}$ beyond the absorbing domain $\mathcal{H}_{\eta, \rho}$. The absorbing domain $\mathcal{H}_{\eta, \rho}$ describes a domain unbounded to the left. The curves $\Gamma_{k, \mu}$ glue to $\mathcal{H}_{\eta, \rho}$ a set of strips unbounded to the right as described above in the remark.

Even more, the curves $\left\{\Gamma_{k, \mu}\right\}_{k}$ are helpful to construct a region where the dynamics of $g_{\lambda, \mu}$ "behave well". Consider the curves $\Gamma_{k, \mu}$ and $\Gamma_{-k, \mu}$ for some large $k \in \mathbb{N}$. We use these two curves to construct a domain $V$ where the triple $\left(g_{\lambda, \mu}, V, g_{\lambda, \mu}(V)\right)$ is polynomial-like. Firstly, we construct a domain $E$ bounded by two consecutive curves from the family $\left\{\Gamma_{k, \mu}\right\}_{k}$ placed w.l.o.g. in the upper half-plane. Later we restrict to a smaller domain $\widetilde{E} \subset \subset E$, such that $\left.g_{\lambda, \mu}\right|_{\widetilde{E}}$ is a proper map. The boundary of $E$ and $\widetilde{E}$ are built up of four curves as we sketch in Figure 2.14 for the domain $E$. Then we use the upper boundary component of $\widetilde{E}$ and the lower boundary component of an analogue set below the real axis to construct a large set $V$ on which we define a polynomial-like triple.

We present in Theorem 2.39 the construction of the domain $V$ and use the result of Lemma 2.40 presented below to prove that $\left.g_{\lambda, \mu}\right|_{V}$ is a proper map. The proof of 
Theorem 2.39 is written at the end of the section. In the proofs in this section we use the notation $\widetilde{\Gamma}_{d}$ instead of $\widetilde{\Gamma}_{d, \mu}$, that is without the parameter $\mu$, for simplicity. Keep in mind, however, that the proofs and arguments depend on the parameter $\mu$ as well as on the parameter $\lambda$.

Theorem 2.39 (Polynomial-like Mapping). For every $d \in \mathbb{N}$ with $d \geq 2$ and any given $\lambda \in \mathbb{C}^{*} \backslash \mathbb{R}^{+}$there exists a sufficiently small $\mu_{0} \in\left(0, \frac{1}{2}\right)$ and a domain $V$ such that the critical points $c_{k} \in V$ for $-d<k<d$ and the triple $\left(g_{\lambda, \mu}, V, g_{\lambda, \mu}(V)\right)$ is a polynomial-like mapping of degree at most $2 d$ for every $\mu \leq \mu_{0}$.

We present here the main tool used in the proof. We start by constructing a smaller domain $E$ where the function $g_{\lambda, \mu}$ is univalent by describing the curves that form its boundary. We obtain that the image of $\bar{E}$ under $g_{\lambda, \mu}$ is not relatively compact in $g_{\lambda, \mu}(E)$, since it contains two critical points. Hence, we consider a slightly smaller domain $\widetilde{E}$ and prove that $g_{\lambda, \mu}(\widetilde{E}) \subset \subset \widetilde{E}$ by means of the Riemann Mapping Theorem and Schwarz Lemma on the unit disc.

Lemma 2.40. Let $E$ be a domain bounded by $\widetilde{\Gamma}_{d} \subset \Gamma_{d, \mu}$ and $\widetilde{\Gamma}_{d-1} \subset \Gamma_{d-1, \mu}$ above and below and by the line segments joining $\widetilde{\Gamma}_{d}$ with $\widetilde{\Gamma}_{d-1}$ to the left and right, denoted by $L_{d}$ and $K_{d}$ respectively. Then any domain $\widetilde{E} \subset \subset E$ is mapped onto a larger domain $g_{\lambda, \mu}(\widetilde{E})$ satisfying $\widetilde{E} \subset \subset g_{\lambda, \mu}(\widetilde{E}) \subset \subset g_{\lambda, \mu}(E)$.

Proof. Let $\mu \in\left(0, \frac{1}{2}\right)$ be a sufficiently small parameter which is arbitrary but fixed such that the invariance of the absorbing domain is satisfied. Recalling the structure theorem we denote by $d$ the largest index for which the Jordan curves $\left\{\Gamma_{k, \mu}\right\}_{k}$ are well defined for all $|k| \leq d, k \neq 0$. We may assume that $d>1$.

Consider two consecutive Jordan curves $\Gamma_{d-1}$ and $\Gamma_{d}$. We construct the domain $E$ by giving its boundary components. Delimit the domain $E$ above by a subset of $\Gamma_{d}$ and below by a subset of $\Gamma_{d-1}$ both bounded as follows. The left boundary points are choose as the points $z_{d} \in \Gamma_{d}$, respectively $z_{d-1} \in \Gamma_{d-1}$, such that $\operatorname{Re} z_{d}=\operatorname{Re} z_{d-1}=1-\frac{1}{2 \mu}$. Now we construct the boundary on the right. Since the Jordan curves are invariant under $g_{\lambda, \mu}$, set $w_{d}:=g_{\lambda, \mu}\left(z_{d}\right)$ which lies as well on $\Gamma_{d}$. Furthermore, the curves are mapped two-to-one by the function $g_{\lambda, \mu}$ onto itself. Therefore the point $w_{d}$ has two different preimages under the corresponding inverse branch of $g_{\lambda, \mu}$. We denote by $\tilde{z}_{d}$ the other preimage of $w_{d}$ and remark that $\operatorname{Re} w_{d}<\operatorname{Re} z_{d}<\operatorname{Re} v_{d}<\operatorname{Re} c_{d}<\operatorname{Re} \tilde{z}_{d}$, where $c_{d}$ and $v_{d}$ are the critical point and value lying on $\Gamma_{d}$. Similarly we define the points $w_{d-1}, z_{d-1}$ and $\tilde{z}_{d-1}$ on $\Gamma_{d-1}$.

For the right end on the curve $\widetilde{\Gamma}_{d}$, we choose the point $\zeta_{d} \in \Gamma_{d}$ with the property that $\operatorname{Re} \zeta_{d}=\max \left\{x_{+}, \operatorname{Re} \tilde{z}_{d}\right\}$, where $x_{+}$is a large positive number satisfying

i) $|\lambda| x_{+}>1$, and 
ii) $x_{+}>\operatorname{Im} \zeta_{d}$

Note that if $\operatorname{Re} \tilde{z}_{d} \geq x_{+}$, then the point $\widetilde{z}_{d}$ still holds conditions i) and ii), which means $\zeta_{d}=\tilde{z}_{d}$. Finally we define $\zeta_{d-1}$ on $\Gamma_{d-1}$ such that $\operatorname{Re} \zeta_{d-1}=\operatorname{Re} \zeta_{d}$.

With the conditions mentioned above, we define the boundary of $E$ as the union of the four curves $\widetilde{\Gamma}_{d} \cup K_{d} \cup \widetilde{\Gamma}_{d-1} \cup L_{d}$, where

i) The upper boundary $\widetilde{\Gamma}_{d} \subset \Gamma_{d}$ goes from $z_{d}$ to $\zeta_{d}$.

ii) The lower boundary $\widetilde{\Gamma}_{d-1} \subset \Gamma_{d-1}$ goes from $z_{d-1}$ to $\zeta_{d-1}$.

iii) On the right side $K_{d}$ is the line segment joining $\zeta_{d}$ with $\zeta_{d-1}$.

iv) On the left side $L_{d}$ is the line segment joining $z_{d}$ with $z_{d-1}$.

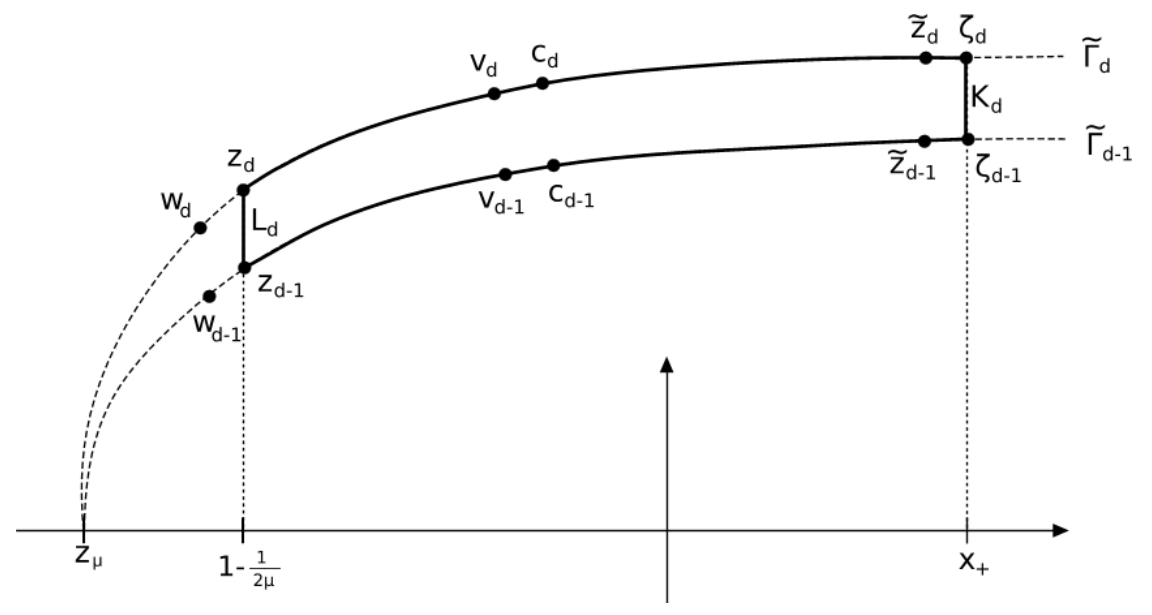

Figure 2.14: Domain $E$ bounded above and below by the curves $\widetilde{\Gamma}_{d}$ and $\widetilde{\Gamma}_{d-1}$. The left and right boundary components are the lines $L_{d}$ and $K_{d}$ respectively.

We describe now the image of $E$ under the function $g_{\lambda, \mu}$. We claim that $g_{\lambda, \mu}\left(K_{d}\right)$ is contained in $\mathbb{C} \backslash \overline{D\left(0,2 x_{+}\right)}$. The value $x_{+}$was chosen to be large enough that $x_{+}>\operatorname{Im} \zeta_{d}$. Hence $x_{+}>\operatorname{Im} z$ for all $z \in \widetilde{\Gamma}_{d}$ as for all $z \in K_{d}$. It is also true that $\operatorname{Im} \zeta_{d}>2 \pi$ for any $d>1$ as it follows from properties i) and $i v$ ) in Theorem 2.21. Then $x_{+}>2 \pi$ and $\left|e^{z}\right| \geq e^{x_{+}}>6 x_{+}$. Moreover, for every $z \in K_{d}$ we obtain that 


$$
\begin{aligned}
& |z-1| \leq|x-1|+|y|<|x|+\left|\operatorname{Im} \zeta_{d}\right| \leq 2 x_{+} \text {. Then } \\
& \qquad \begin{aligned}
\left|g_{\lambda, \mu}(z)\right| & =(1-\mu)\left|z-1+\lambda z e^{z}\right| \\
\geq & (1-\mu)\left(\left|\lambda z e^{z}\right|-|z-1|\right) \\
& >(1-\mu)\left(6 x_{+}-2 x_{+}\right)>2 x_{+} \quad \text { for all } \mu \in\left(0, \frac{1}{2}\right) .
\end{aligned}
\end{aligned}
$$

The image of $K_{d}$ describes a large Jordan arc connecting $\xi_{d}:=g_{\lambda, \mu}\left(\zeta_{d}\right)$ with $\xi_{d-1}:=g_{\lambda, \mu}\left(\zeta_{d-1}\right)$. This is true, since $K_{d}$ is a straight line with sufficiently large positive real part and does not contain any singular value. In particular, we have that $\left|z_{\mu}\right|>\left|g_{\lambda, \mu}\left(\zeta_{d}\right)\right|>2 x_{+}$, where $z_{\mu}$ denotes the attracting fixed point.

Using the structure theorem again we prove that the curve $\widetilde{\Gamma}_{d}$ is mapped two-toone into $\Gamma_{d}$ such that $v_{d}$ is the boundary point at the right end of $g_{\lambda, \mu}\left(\widetilde{\Gamma}_{d}\right)$. The image of left and right ends of $\widetilde{\Gamma}_{d}$ coincide at $\xi_{d}$, the left end of $g_{\lambda, \mu}\left(\widetilde{\Gamma}_{d}\right)$. In other words, the image of $\widetilde{\Gamma}_{d}$ goes from $v_{d}$ to $\xi_{d}$. Recall that $\operatorname{Re} \xi_{d} \leq \operatorname{Re} w_{d}=\operatorname{Re} g_{\lambda, \mu}\left(\tilde{z}_{d}\right)$. Similarly, $g_{\lambda, \mu}\left(\widetilde{\Gamma}_{d-1}\right)$ connects $v_{d-1}$ with $\xi_{d-1}$ as we sketch in Figure 2.15. Finally, for all $z \in L_{d}$ it follows that $\operatorname{Re} g_{\lambda, \mu}(z)<\operatorname{Re} z$ since $L_{d} \subset \partial \mathcal{H}_{R e}$; see Lemma 2.31.

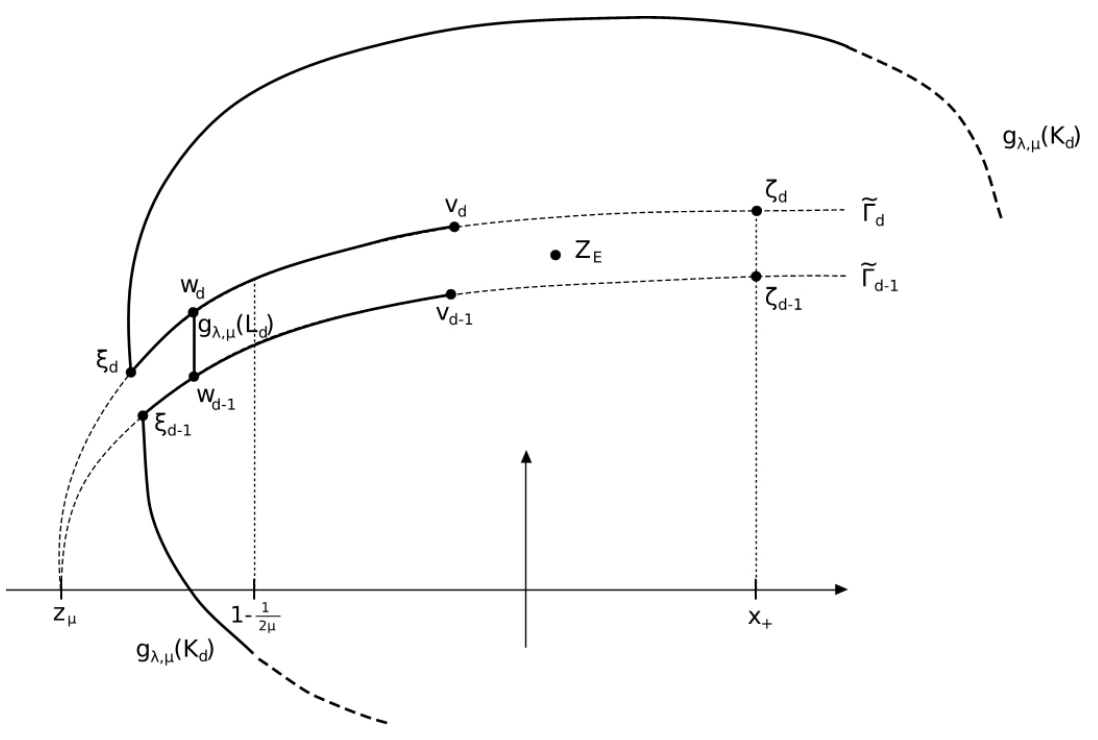

Figure 2.15: Image of domain $E$ under the function $g_{\lambda, \mu}$. The curve $g_{\lambda, \mu}\left(K_{d}\right) \subset \mathbb{C} \backslash \overline{D\left(0,2 x_{+}\right)}$is a large Jordan arc connecting $\xi_{d}=g_{\lambda, \mu}\left(\zeta_{d}\right)$ with $\xi_{d-1}=g_{\lambda, \mu}\left(\zeta_{d-1}\right)$. We remark that the intersection of $g_{\lambda, \mu}\left(\widetilde{\Gamma}_{d}\right)$ with $\widetilde{\Gamma}_{d}$ is not empty. 
The domain $E$ is simply connected and $\left.g_{\lambda, \mu}\right|_{E}$ is univalent. Thus $g_{\lambda, \mu}(E)$ is also simply connected and $g_{\lambda, \mu}(\partial E)$ is connected. Consider now the domain $g_{\lambda, \mu}(E)$ and the inverse branch of $g_{\lambda, \mu}$ that leaves the strip in between $\Gamma_{d}$ and $\Gamma_{d-1}$ invariant. We obtain that $g_{\lambda, \mu}(E)$ is mapped by $g_{\lambda, \mu}^{-1}$ to $E$, a smaller domain and containing two critical points on the boundary. Then there exists a fixed point in $E$, say $z_{E}$, which is attracting with respect to $g_{\lambda, \mu}^{-1}$.

Hence, there exists a Riemann mapping $\varphi: g_{\lambda, \mu}(E) \rightarrow \mathbb{D}$ with $\varphi\left(z_{E}\right)=0$ which can be holomorphically extended to a map $\varphi: \overline{g_{\lambda, \mu}(E)} \rightarrow \overline{\mathbb{D}}$. Let $\psi: \mathbb{D} \rightarrow g_{\lambda, \mu}(E)$ denote the pull-back of $\varphi$, so that the following diagram commutes.

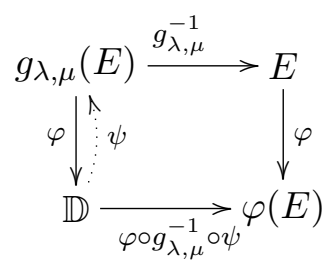

We describe the distribution of the marked points on $\overline{\mathbb{D}}$ after the projection. The marked points of $g_{\lambda, \mu}\left(\Gamma_{d}\right)$ go under the action of $\varphi$ to the points $w_{d}^{\prime}, \varphi\left(z_{d}\right), \varphi\left(v_{d}\right)$, $w_{d}^{\prime \prime}$ and $\varphi\left(\xi_{d}\right)$ lying on $\partial \mathbb{D}$ clockwise in this order. Under the pull-back $\psi$ we obtain $\psi\left(w_{d}^{\prime}\right)=w_{d}=\psi\left(w_{d}^{\prime \prime}\right)$. Analogously for the curve $g_{\lambda, \mu}\left(\widetilde{\Gamma}_{d-1}\right)$ we label the image of the marked points under $\varphi$ such that $w_{d-1}^{\prime}, \varphi\left(z_{d-1}\right), \varphi\left(v_{d-1}\right), w_{d-1}^{\prime \prime}$ and $\varphi\left(\xi_{d-1}\right)$ lie in this order counter-clockwise on $\partial \mathbb{D}$ and $\psi\left(w_{d-1}^{\prime}\right)=w_{d-1}=\psi\left(w_{d-1}^{\prime \prime}\right)$.

The point $\varphi\left(g_{\lambda, \mu}\left(z_{E}\right)\right)=0$ is an attracting fixed point. Using Schwarz Lemma, the function $\varphi \circ g_{\lambda, \mu}^{-1} \circ \psi$ maps $\mathbb{D}=\varphi\left(g_{\lambda, \mu}(E)\right)$ into a smaller domain $\varphi(E)$ acting as a contraction on $\mathbb{D}$. Note, however, that $\partial \varphi\left(g_{\lambda, \mu}(E)\right) \cap \partial \varphi(E) \neq \emptyset$ and that the intersection is a subset of $\partial \mathbb{D}$.

What does $\varphi(E)$ look like? We answer this question again through the description of the boundary of $\varphi(E)$ and refer the reader to Figure 2.16 for a better understanding. A first boundary curve lies in $\mathbb{D}$ joining the point $\varphi\left(z_{d-1}\right)$ with $\varphi\left(z_{d}\right)$. Later the boundary continues with an arc segment on $\partial \mathbb{D}$ joining $\varphi\left(z_{d}\right)$ with $\varphi\left(v_{d}\right)$, where the latter equals the intersection of $\varphi\left(g_{\lambda, \mu}\left(\widetilde{\Gamma}_{d}\right)\right) \cap \varphi\left(\widetilde{\Gamma}_{d}\right)$. The boundary carries forward with a curve which lies in $\mathbb{D}$ joining $\varphi\left(v_{d}\right)$ with $\varphi\left(c_{d}\right)$ and further going through $\varphi\left(\zeta_{d}\right), \varphi\left(\zeta_{d-1}\right)$ and $\varphi\left(c_{d-1}\right)$ until it again reaches $\partial \mathbb{D}$ at $\varphi\left(v_{d-1}\right)$. The boundary closes with the arc segment connecting $\varphi\left(v_{d-1}\right)$ with $\varphi\left(z_{d-1}\right)$ on $\partial \mathbb{D}$. Similarly it holds that $\varphi\left(v_{d-1}\right)$ equals the intersection of $\varphi\left(g_{\lambda, \mu}\left(\widetilde{\Gamma}_{d-1}\right)\right)$ with $\varphi\left(\widetilde{\Gamma}_{d-1}\right)$.

Finally, we proceed to define the smaller domain $\widetilde{E} \subset E$. Take any closed disc 


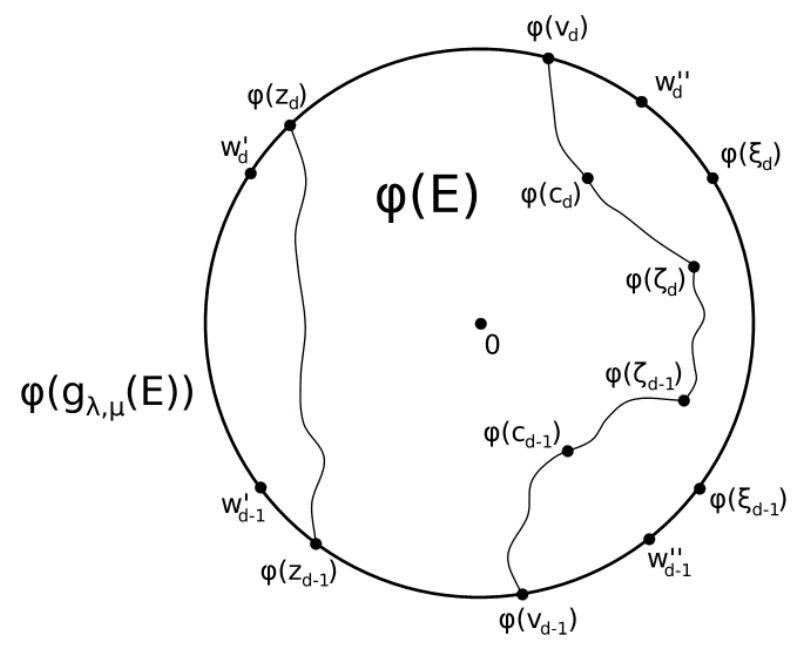

Figure 2.16: The Riemann mapping $\varphi$ projects the marked points of $\partial g_{\lambda, \mu}(E)$ on $\partial \mathbb{D}$ as described in the figure. The curves and points distinguished in $\mathbb{D}$ are part of $\partial \varphi(E)=\partial\left(\left(\varphi \circ g_{\lambda, \mu}^{-1} \circ \psi\right)(\mathbb{D})\right)$.

$\overline{D_{r}}:=\{z \in \mathbb{C}:|z| \leq r\}$ with $r<1$. Then $\overline{D_{r}}$ is mapped strictly inside $D_{r}$, that is, $\left(\varphi \circ g_{\lambda, \mu}^{-1} \circ \psi\right)\left(\overline{D_{r}}\right)$ is relatively compact in $D_{r}$. Consider the pull-back of the disc and later its image under $g_{\lambda, \mu}^{-1}$. It follows that $\psi\left(\overline{D_{r}}\right) \subset g_{\lambda, \mu}(E)$ and $\left(g_{\lambda, \mu}^{-1} \circ \psi\right)\left(\overline{D_{r}}\right) \subset E$. We define $\partial g_{\lambda, \mu}(\widetilde{E}):=\psi\left(\partial D_{r}\right)$. Its image under $g_{\lambda, \mu}^{-1}$ defines the curve $\partial \widetilde{E}$, which is strictly contained in $E$. In particular, we have $\left(\varphi \circ g_{\lambda, \mu}^{-1} \circ \psi\right)\left(\overline{D_{r}}\right)=\varphi(\widetilde{E}) \subset \subset \varphi(E)$, as a consequence of the contraction in $\mathbb{D}$.

From the point of view of the function $g_{\lambda, \mu}$, we defined an arbitrary domain $\widetilde{E}$ relatively compact in $E$ which boundary is mapped outside of $\widetilde{E}$ but inside of $g_{\lambda, \mu}(E)$ yielding the desired result.

As a conclusion, note that if we choose a radius $r$ sufficiently close to one, then $\psi\left(\partial D_{r}\right)=\partial g_{\lambda, \mu}(\widetilde{E})$ is very close to $\partial g_{\lambda, \mu}(E)$. Part of the boundary of $\widetilde{E}$ is a curve very close to $\widetilde{\Gamma}_{d}$ (resp. a curve close to $\widetilde{\Gamma}_{d-1}$ ) inside of $E$ which is mapped by $g_{\lambda, \mu}$ "surrounding" $g_{\lambda, \mu}\left(\widetilde{\Gamma}_{d}\right)$ (resp. $\left.g_{\lambda, \mu}\left(\widetilde{\Gamma}_{d-1}\right)\right)$ and inside of $g_{\lambda, \mu}(E)$. This is as a consequence of the fact that $g_{\lambda, \mu}$ maps $\widetilde{\Gamma}_{d}$ two-to-one onto it self, but $g_{\lambda, \mu}$ is univalent in the interior of $E$. In particular, we have that $g_{\lambda, \mu}$ is a one-to-one mapping in $\widetilde{E}$ and on $\partial \widetilde{E}$, that is $\left.g_{\lambda, \mu}\right|_{\widetilde{E}}$ is a proper map.

Remark 2.2. The proof of Lemma 2.40 depends on the first hand on the parameters $\mu$ and $\lambda$, and on the invariance of the curves $\Gamma_{k}$ and the absorbing domain. On the other hand, the arguments to prove that $g_{\lambda, \mu}$ is a proper map restricted 
to $\widetilde{E}$ heavily depends on the value $x_{+}$which is defined to be a large positive number. Furthermore, for a radius $r$ sufficiently close to one, we guarantee that $\partial \widetilde{E}$ contains no critical values. Therefore, the proof applies in a similar way to any given $d \in \mathbb{Z}$ with $|d|>1$ and for any pair of subsequent curves $\Gamma_{d}$ and $\Gamma_{d-1}$ or analogously $\Gamma_{-d}$ and $\Gamma_{-d+1}$ below the real axis.

Recall that a triple $(f, A, B)$ is a polynomial-like mapping if the sets $A$ and $B$ are bounded simply connected domains satisfying $\bar{A} \subset B$ and $f: A \rightarrow B$ is a holomorphic proper map. With this we proceed to prove the theorem. At first we construct a domain $U$ for given $\mu_{0}$ and $d$ by means of the Structure Theorem 2.37 and Lemma 2.40. Afterwards we take the restriction of $g_{\lambda, \mu}$ to a domain $V \subset U$ constructed in such a way that $V$ is simply connected and $\bar{V} \subset g_{\lambda, \mu}(V)$ implying that $\left.g_{\lambda, \mu}\right|_{V}$ is a proper map.

Proof of Theorem 2.39. Let $\mu \in\left(0, \frac{1}{2}\right)$ be sufficiently small that the existence of the absorbing domain $\mathcal{H}_{\eta, \rho}$ is guaranteed. Furthermore let $d$ be the largest index such that the family of Jordan curves $\left\{\Gamma_{k, \mu}\right\}_{k}$ is well defined for every $|k| \leq d, k \neq 0$ as described in the Structure Theorem 2.37. Assume without loss of generality that $d>1$.

We proceed with the construction of $\partial U$ by considering the curves $\Gamma_{d}$ and $\Gamma_{-d}$. We mark the points $z_{d} \in \Gamma_{d}$ and $z_{-d} \in \Gamma_{-d}$ satisfying $\operatorname{Re} z_{d}=1-\frac{1}{2 \mu}=\operatorname{Re} z_{-d}$. Define the points $\zeta_{d} \in \Gamma_{d}$ and $\zeta_{-d} \in \Gamma_{-d}$ with the properties $\operatorname{Re} \zeta_{d}=\max \left\{x_{+}, \operatorname{Re} \tilde{z}_{d}\right\}$ and $\operatorname{Re} \zeta_{-d}=\max \left\{x_{+}, \operatorname{Re} \tilde{z}_{-d}\right\}$, where

i) $g_{\lambda, \mu}\left(\tilde{z}_{d}\right)=w_{d}=g_{\lambda, \mu}\left(z_{d}\right)$,

ii) $g_{\lambda, \mu}\left(\tilde{z}_{-d}\right)=w_{-d}=g_{\lambda, \mu}\left(z_{-d}\right)$,

iii) $x_{+}$is large enough that $|\lambda| x_{+}>1$, and

iv) $x_{+}>\max \left\{\left|\operatorname{Im} \zeta_{d}\right|,\left|\operatorname{Im} \zeta_{-d}\right|\right\}$,

as in the proof of Lemma 2.40. We restrict the curves $\Gamma_{d}$ and $\Gamma_{-d}$ to the segments going from $z_{d}$ to $\zeta_{d}$ and from $z_{-d}$ to $\zeta_{-d}$, and denote them by $\widetilde{\Gamma}_{d}$ and $\widetilde{\Gamma}_{-d}$ respectively.

We define the right component of $\partial U$ by the line segment $K$ joining $\zeta_{d}$ with $\zeta_{-d}$. The left component of $\partial U$ consists of the line segment $L \subset\left\{z \in \mathbb{C}: \operatorname{Re} z=1-\frac{1}{2 \mu}\right\}$ joining $z_{d}$ with $z_{-d}$. Finally we define $\partial U:=L \cup \widetilde{\Gamma}_{d} \cup K \cup \widetilde{\Gamma}_{-d}$.

The curves $\widetilde{\Gamma}_{1}$ and $\widetilde{\Gamma}_{-1}$ are contained in $U$ and hence there exists at least two critical points and their values in $U$. Let $c_{ \pm d}$ denote the critical points lying on the curves $\Gamma_{ \pm d}$ respectively. Then by Theorem 2.21 we have $\left|\operatorname{Im} c_{ \pm d}\right|>2 \pi$. In 
particular, the latter implies $2 \pi<\left|\operatorname{Im} c_{ \pm d}\right|<|\operatorname{Im} z|$ for all $z \in \Gamma_{ \pm d}$ such that $\operatorname{Re} c_{ \pm d}<\operatorname{Re} z$.

Now we describe the image of $U$ under the function $g_{\lambda, \mu}$ by means of the result in Lemma 2.40 that describes $g_{\lambda, \mu}(\partial E)$. For points $z$ lying on $L$ we obtain that $\operatorname{Re} g_{\lambda, \mu}(z)<\operatorname{Re} z$. Furthermore, for points $z \in K$ we have by definition that $|z| \leq \max \left\{\left|\zeta_{d}\right|,\left|\zeta_{-d}\right|\right\}$. Then $g_{\lambda, \mu}(K)$ describes a large arc going from $\xi_{d}$ to $\xi_{-d}$, but, in contrast to the construction of the domain $E$, this arc is not simple. The number of times the $\operatorname{arc} g_{\lambda, \mu}(K)$ twists around $U$ depends on the number of critical points contained in $U$ in terms of the Residue Theorem. Then it twists at most $2 d-1$ times, counting the critical point $c_{0}$ that lies in a neighbourhood of the real axis using Property $i v$ ) in Theorem 2.21. The construction of the points $\zeta_{d}$ and $\zeta_{-d}$ guarantees for all $z \in K$ that $\left|g_{\lambda, \mu}(z)\right|>2 x_{+}$. Hence $g_{\lambda, \mu}(K)$ is mapped outside the disc $D\left(0,2 x_{+}\right)$implying that $g_{\lambda, \mu}(K) \cap g_{\lambda, \mu}(L)=\emptyset$. Finally by applying Lemma 2.40 again, we conclude that the image of $\widetilde{\Gamma}_{d}$ (resp. $\widetilde{\Gamma}_{-d}$ ) goes from $\xi_{d}$ to $v_{d}$ (resp. from $\xi_{-d}$ to $v_{-d}$ ). In other words, the curves are mapped by $g_{\lambda, \mu}$ two-to-one overlapping onto itself.

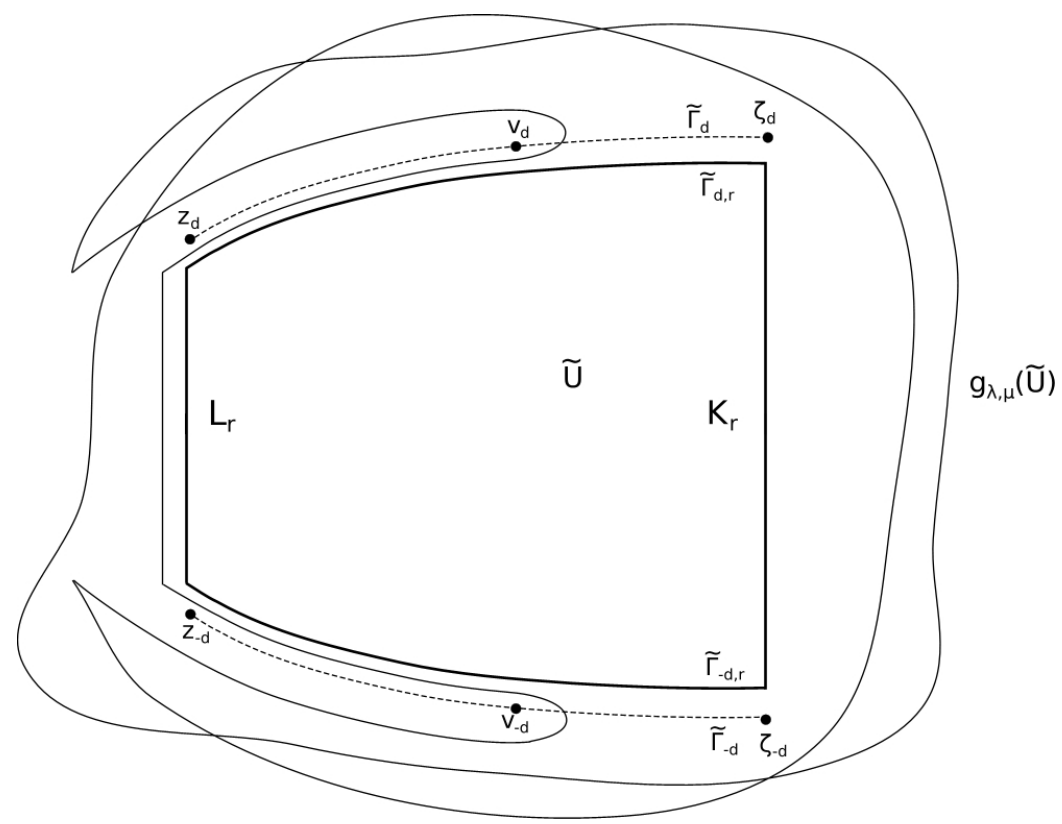

Figure 2.17: The domain $\widetilde{U}$ is defined through its boundary $\partial \widetilde{U}:=L_{r} \cup$ $\widetilde{\Gamma}_{d, r} \cup K_{r} \cup \widetilde{\Gamma}_{-d, r}$. The outer non-simple closed curve sketches the image $g_{\lambda, \mu}(\partial \widetilde{U})$. 
The latter argument implies that $\partial U \cap g_{\lambda, \mu}(\partial U) \neq \emptyset$. Therefore, we define a smaller domain $\widetilde{U} \subset U$ as follows. To this end, recall the pull back of the disc $D_{r}$ for some $r \in(0,1)$ close to one as described in Lemma 2.40. Denote by $\widetilde{\Gamma}_{d, r}$ the curve mapped below $\widetilde{\Gamma}_{d}$ by the action of $g_{\lambda, \mu}^{-1} \circ \psi$ on the corresponding arc segment of $D_{r}$. Analogously we denote by $\widetilde{\Gamma}_{-d, r}$ the curve above $\widetilde{\Gamma}_{-d}$ as it can be seen in Figure 2.17. The left side of the boundary is denoted by $L_{r} \subset L$ and has as corner points the intersection of the curves $\widetilde{\Gamma}_{ \pm d, r}$ with the line $L$. Similarly we define the right component of the boundary denoted by $K_{r} \subset K$. In conclusion, we have

$$
\partial \widetilde{U}:=L_{r} \cup \widetilde{\Gamma}_{d, r} \cup K_{r} \cup \widetilde{\Gamma}_{-d, r} .
$$

We remark that by construction $\partial \widetilde{U} \cap g_{\lambda, \mu}(\partial \widetilde{U})=\emptyset$. However, $g_{\lambda, \mu}(\widetilde{U})$ may not be simply connected, since the image of $K_{r}$ is not a simple arc. Therefore we define the domain $V^{\prime} \subset g_{\lambda, \mu}(\widetilde{U})$ as the simply connected component of $\mathbb{C} \backslash g_{\lambda, \mu}(\partial \widetilde{U})$ that contains $\widetilde{U}$. Denote by $V$ the preimage of $V^{\prime}$ under the function $g_{\lambda, \mu}$. The map $\left.g_{\lambda, \mu}\right|_{V}$ is proper and the triple $\left(g_{\lambda, \mu}, V, V^{\prime}\right)$ is polynomial-like with $g_{\lambda, \mu}(V)=V^{\prime}$. Finally notice that there are exactly $2(d-1)+1$ critical points contained in $V$ for $r$ sufficiently close to one and $\lambda \in \mathbb{C}^{*} \backslash \mathbb{R}^{+}$. Hence the degree of $\left(g_{\lambda, \mu}, V, V^{\prime}\right)$ equals $2 d$ using the Riemann-Hurwitz Formula for domains, presented in the introduction as Lemma 1.4 .

A different point of view of Theorem 2.39 is expressed with respect to an arbitrarily large domain in $\mathbb{C}$ and not depending on the degree of the polynomial-like mapping.

Corollary 2.41. For every given bounded domain $M \subset \mathbb{C}$ there exist $d \in \mathbb{N}$ and a domain $V$ with $V \supset M$ such that the triple $\left(g_{\lambda, \mu}, V, g_{\lambda, \mu}(V)\right)$ is polynomial-like of degree $2 d$.

Proof. For any given domain $M \subset \subset \mathbb{C}$, let $M^{\prime}$ be a bounded domain containing $M$ and $g_{\lambda, \mu}(M)$. Let $\mu \in\left(0, \frac{1}{2}\right)$ be sufficiently small that $z_{\mu}<\operatorname{Re} z$ for every $z \in M^{\prime}$ and that the existence of the absorbing domain $\mathcal{H}_{\eta, \rho}$ is guaranteed.

Let $d \in \mathbb{N}$ be such that the family of Jordan curves $\left\{\Gamma_{k, \mu}\right\}_{k}$ is well defined for every $|k| \leq d, k \neq 0$ and set $\zeta_{ \pm d} \in \Gamma_{ \pm d}$ with equal real parts and satisfying

$$
\operatorname{Im} \zeta_{-d}<\operatorname{Im} z<\operatorname{Im} \zeta_{d}
$$

for every $z \in M^{\prime}$. Furthermore, consider the value $x_{+}$large enough with the additional condition that $x_{+}>\operatorname{Re} z$ for all $z \in M^{\prime}$. If the assumptions with 
respect to the real and imaginary parts of $z \in M^{\prime}$ are not satisfied, we may take a smaller value of $\mu$. Then the result follows immediately from Theorem 2.39 which yields a domain $U \supset \supset M^{\prime}$ with the values $x_{+}$and $d$ as described above.

The family of Jordan curves enables us to see more of the structure of the basin of attraction. Besides the domain $\mathcal{H}_{\eta, \rho}$, that is unbounded to the left, the basin of attraction also contains countable many strips unbounded to the right. Furthermore, using the Straightening Theorem, the function $g_{\lambda, \mu}$ restricted to $V$ is hybrid equivalent to a polynomial of degree $2 d$.

We can see that the Julia set is contained in the "gaps" in between these strips. Even though the Julia set $\mathcal{J}\left(g_{\lambda, \mu}\right)$ exhibits a lot of the structure known as Cantor bouquets intrinsic of functions having an exponential component, the function $g_{\lambda, \mu}$ has a structure much more intricate as than expected. However, the convergence of the Julia sets holds. We describe the Julia set of $g_{\lambda, \mu}$ and its convergence to $\mathcal{J}\left(g_{\lambda}\right)$ more in the detail in Chapter 3 . 


\section{Convergence of the families $g_{\lambda, \mu}$ to $g_{\lambda}$}

This chapter focuses mainly on the approximation in a dynamical sense of the family of transcendental functions $g_{\lambda}(z)=z-1+\lambda z e^{z}$ by $g_{\lambda, \mu}(z)=(1-\mu)(z-$ $\left.1+\lambda z e^{z}\right)$ for every $\lambda \in \mathbb{C}^{*}$ and $\mu \in\left(0, \frac{1}{2}\right)$ as $\mu$ tends to 0 .

Hinkkanen et al, 33] began investigating the transcendental entire function $g_{\lambda}$ for the parameter $\lambda=-2 e^{1 / 2}$. Using a different parametrisation of $g_{\lambda}$ they showed that the function features the origin as a superattracting fixed point together with a Baker domain. Afterwards, Lauber continued in [48] with the analysis for arbitrary $\lambda \in \mathbb{C}^{*}$. He proved that the Fatou set of $g_{\lambda}$ contains a unique Baker domain $\mathcal{B}_{\lambda}$ for every $\lambda$ and for some parameters also an attracting basin. In general, $g_{\lambda}$ has at most one free critical value; that is, $\mathcal{F}\left(g_{\lambda}\right)$ contains at most one non-repelling cycle besides $\mathcal{B}_{\lambda}$.

In Chapter 2 we proved that the Fatou set of $g_{\lambda, \mu}$ has an attracting basin $\mathcal{A}_{\mu}$ for every $\lambda \in \mathbb{C}^{*}$ and for every $0<\mu \leq \mu_{0}$ with sufficiently small $\mu_{0}=\mu_{0}(\lambda)$. The attracting fixed point $z_{\mu}$ is contained in the disc $D\left(1-\frac{1}{\mu}, 1\right)$ and $z_{\mu} \rightarrow-\infty$ as $\mu \rightarrow 0$. In order to give a more detailed description of the basin of attraction, we constructed an invariant absorbing domain $\mathcal{H}_{\eta, \rho} \subset \mathcal{A}_{\mu}$ which is unbounded to the left. One of the main results in this chapter is the convergence of the attracting basins $\mathcal{A}_{\mu}$ to the Baker domain $\mathcal{B}_{\lambda}$ for every fixed parameter $\lambda$ as $\mu \rightarrow 0$. In order to show this convergence we prove that the absorbing domains $\mathcal{H}_{\eta, \rho}$ converge as kernels in the sense of Carathéodory to the absorbing domain $\mathcal{M}_{\delta}$ of $\mathcal{B}_{\lambda}$.

In Section 2.5 we described the dynamics in the basin of attraction $\mathcal{A}_{\mu}$ for points 
with large positive real part by constructing a family of invariant Jordan curves $\left\{\Gamma_{k, \mu}\right\}_{k}$. These Jordan curves imply the existence of open regions which are asymptotically horizontal as the real part increases to $\infty$. An analogue to this result for $g_{\lambda}$ enables us to prove that $\mathcal{F}\left(g_{\lambda}\right)$ has no wandering domains if the free critical point belongs to the Baker domain $\mathcal{B}_{\lambda}$ or to an attracting basin $\mathcal{A}_{0}$; see Theorem 3.6. In the case that the free critical point belongs to another non-repelling cycle different from $\mathcal{A}_{0}$, the non-existence of wandering domains follows, too, as we prove in Corollary 3.8 . Unfortunately, we are not able to extend this result to the function $g_{\lambda, \mu}$, since we do not have control on the critical points with large imaginary part.

In the first section we prove the non-existence of wandering domains for the function $g_{\lambda}$ and present some other results obtained previously by Lauber, which are required in the subsequent sections. Section 3.3 is devoted to prove the kernel convergence as $\mu$ tends to zero. We firstly show the convergence of the attracting basins $\mathcal{A}_{\mu}$ to Baker domain $\mathcal{B}_{\lambda}$, and secondly of the basins of attraction $\mathcal{A}_{p, \mu}$ to the parabolic basin $\mathcal{D}_{p}$ in the case that $g_{\lambda}$ has a parabolic fixed point $z_{p}$. For this aim, we prove in Section 3.2 the approximation of any indifferent fixed point $z_{p}$ by attracting fixed points $z_{p, \mu}$ of $g_{\lambda, \mu}$. In Section 3.4 we consider parameters for which the free critical point $c_{0}$ of $g_{\lambda}$ is neither in $\mathcal{B}_{\lambda}$ nor in a parabolic basin $\mathcal{D}_{p}$. For these parameters $\lambda$ we define filled Julia sets $\mathcal{K}\left(g_{\lambda}\right)$ and $\mathcal{K}\left(g_{\lambda, \mu}\right)$ and prove their Hausdorff convergence for $\mu \leq \mu_{*}$. Finally, we use most of the results presented until then to prove the Hausdorff convergence of $\mathcal{J}\left(g_{\lambda, \mu}\right)$ to $\mathcal{J}\left(g_{\lambda}\right)$ as $\mu \rightarrow 0$ in Section 3.5 .

It is important to note that a family of functions having a Baker domain, such as $g_{\lambda}$, is approximated by a family of transcendental functions $g_{\lambda, \mu}$. This is a new approach as found in the literature, where the transcendental functions are approximated by polynomials.

\subsection{Some results about the family $g_{\lambda}$}

In this section we prove the non-existence of wandering domains for functions $g_{\lambda}$ having either only the Baker domain $\mathcal{B}_{\lambda}$ or additionally a non-repelling cycle. Furthermore, we present as well some results about this family of functions due to Lauber and found in his Dissertation [48] which are useful for the convergence and the results in the rest of the chapter. We present Lauber's results here without proofs and recall the original numeration as a reference. 
During this chapter let $\lambda \in \mathbb{C}^{*}$ and $g_{\lambda}$ be the function defined as

$$
g_{\lambda}(z)=z-1+\lambda z e^{z}
$$

We start by presenting some results about the dynamics of $g_{\lambda}$ and the Baker domain $\mathcal{B}_{\lambda}$.

Theorem 3.1. (Thm. 2) $\mathcal{B}_{\lambda}$ contains all critical points of $g_{\lambda}$ except at most one.

Corollary 3.2. (Cor. 9) Let $c_{0}$ denote the free critical point. Then there exists an $\varepsilon>0$ such that $\operatorname{dist}\left(P\left(g_{\lambda}\right) \backslash O^{+}\left(c_{0}\right), \partial \mathcal{B}_{\lambda}\right)>\varepsilon$.

Proposition 3.3. (Prop. 3) There exists at most one cycle of non-repelling periodic points of $g_{\lambda}$ and $\mathcal{B}_{\lambda}$ is the only Baker domain in $\mathcal{F}\left(g_{\lambda}\right)$.

In the case that $g_{\lambda}$ has an attracting cycle besides $\mathcal{B}_{\lambda}$ we obtain that the critical points are at a positive distance of the Julia set. An approximative geometrical description of the Baker domain is given by the absorbing domain $\mathcal{M}_{1 / 2}$ contained in a left half-plane. In Section 2.5 we construct invariant Jordan curves $\Gamma_{k, \mu}$ for the function $g_{\lambda, \mu}$ which are asymptotically horizontal for points with large positive real part and are contained in the basin of attraction $\mathcal{A}_{\mu}$. In the case of $g_{\lambda}$, the Baker domain presents an analogue structure, as it was proved by Lauber.

Proposition 3.4. (Prop. 4) There exist Jordan curves $\Gamma_{k}, k \in \mathbb{Z} \backslash\{0\}$ such that

i) All $\Gamma_{k}$ are pairwise disjoint.

ii) Each $\Gamma_{k}$ is forward invariant and contained in $\mathcal{B}_{\lambda}$.

iii) Each $\Gamma_{k}$ contains exactly one critical point.

iv) Each $\Gamma_{k}$ is contained in a horizontal strip of height $5 \pi$.

v) Each $\Gamma_{k}$ stretches from $-\infty$ to $\infty$.

Hence there exist "strips" which asymptotically extend horizontally to $+\infty$ outside of the absorbing domain $\mathcal{M}_{1 / 2}$. We refer the reader to Figures 1 and 2 in the first chapter for pictures of these. The latter arguments imply that if there exists a Fatou component besides $\mathcal{B}_{\lambda}$, then it must consist of infinitely many components trapped in small regions. With this, the distance from these components to the Julia set must be bounded. These ideas enable us to use the following result from Hinkkanen et al. [33], which in turn is a modification of Theorem 1.13 due 
to Bergweiler. Recall that $P(f)$ denotes the forward orbit of the set of singular values of $f$.

Lemma 3.5. ([33, Lemma 15]) Let $f$ be a transcendental entire function and $U \subset \mathbb{C}$ be an open subset of the plane invariant with respect to $f$. We assume $P(f) \subset U$ and

$$
\operatorname{dist}(P(f), \partial U):=\inf \{|z-w|: z \in P(f), w \in \partial U \cap \mathbb{C}\}>\sigma
$$

for some positive constant $\sigma$. If there exists $\zeta_{0} \in \mathcal{F}(f)$ such that $f^{n}\left(\zeta_{0}\right) \notin U$ for every $n \in \mathbb{N}$ and

$$
\operatorname{dist}\left(f^{n}\left(\zeta_{0}\right), \partial U\right):=\inf _{z \in \partial U}\left|z-\zeta_{0}\right|<C
$$

for some constant $C>0$, then $\zeta_{0}$ is not contained in a Baker domain nor in a wandering domain of $f$.

We state now the result on wandering domains.

Theorem 3.6. Let $\lambda \in \mathbb{C}^{*}$ be such that $\mathcal{F}\left(g_{\lambda}\right)$ consists of a Baker domain only, or such that the free critical point is in the basin of an attracting periodic cycle. Then $g_{\lambda}$ has no wandering domains.

Proof. Throughout this proof, let $\lambda$ be an arbitrary but fixed parameter satisfying the hypothesis stated in the theorem. If the free critical point $c_{0}$ belongs to the attracting basin of the fixed point $z_{0}$, denote by $\mathcal{A}_{0}$ its immediate basin. In the case that $z_{0}$ is a periodic point, replace $\mathcal{A}_{0}$ by the cycle of immediate basins. Assume without loss of generality that $c_{0} \in \mathcal{A}_{0}$ and denote by $\varepsilon_{0}$ the positive constant defined as $\varepsilon_{0}:=\inf _{z \in O^{+}\left(c_{0}\right)} \operatorname{dist}\left(z, \partial \mathcal{A}_{0}\right)$.

Let $U=\mathcal{B}_{\lambda} \cup \mathcal{A}_{0}$. Then $U$ is an open subset of $\mathbb{C}$ satisfying $P(f) \subset U$. Using Proposition 3.3 , there exists no other Baker domain beside $\mathcal{B}_{\lambda}$. Furthermore, assume that there exists a wandering domain $\mathcal{W}$ and let $\zeta_{0}$ be an arbitrary point in $\mathcal{W}$. Since $\zeta_{0} \notin \mathcal{B}_{\lambda}$ and using Proposition 3.4 we find a constant $C>0$ such that $\operatorname{dist}\left(g_{\lambda}^{n}\left(\zeta_{0}\right), \partial U\right)<C$ for all $n \in N$. Let $\sigma=\min \left\{\varepsilon_{0}, \varepsilon\right\}$, where $\varepsilon$ denotes the distance between $P\left(g_{\lambda}\right) \backslash O^{+}\left(c_{0}\right)$ and $\partial \mathcal{B}_{\lambda}$ as in Corollary 3.2. We have found constants $C$ and $\sigma$ as required in the hypothesis of Lemma 3.5 concluding that $\zeta_{0}$ cannot be contained in a wandering domain.

Lauber proved the following theorem to rule out the existence of wandering domains. We explicitly express in the corollary below the conditions on the function $g_{\lambda}$ such that it has no wandering domains.

Theorem 3.7. (Thm. 3) If $g_{\lambda}$ has a wandering domain $\mathcal{W}$, then 
- there are no non-repelling periodic points, and

- the free critical point exists and is contained in the Julia set, but not in the boundary of a wandering domain (in particular, it is not contained in a wandering domain). The sequence of its iterates has infinitely many accumulation points, including $\infty$.

Corollary 3.8. Let $c_{0}$ denote the free critical point of $g_{\lambda}$. Then exactly one of the following statements holds.

- The critical point $c_{0}$ lies in the Baker domain $\mathcal{B}_{\lambda}$.

- The function $g_{\lambda}$ has a non-repelling cycle different from $\mathcal{B}_{\lambda}$.

- The function $g_{\lambda}$ may (but not need to) have a wandering domain.

Proof. Denote by $P\left(g_{\lambda}\right)^{\prime}$ the derived set of the post-singular set of $g_{\lambda}$, that is the set of all limit points of the forward orbit of the singular values. Write $\mathcal{W}$ for a wandering domain.

We mentioned in the introduction in Theorem 1.18 that all limit functions of $\left\{\left.f\right|_{\mathcal{W}}\right\}$ are constant and contained in $P(f)^{\prime} \cup \infty$. Hence, if the unique free critical point lies in the Baker or an attracting basin, then there can not exist a wandering domain, as we proved above. If $g_{\lambda}$ has an indifferent cycle, either rational or irrational, the limit functions of $\mathcal{W}$ cannot lie in $P\left(g_{\lambda}\right)^{\prime}$ since they belong to the closure of the Fatou component of the non-repelling cycle and remain bounded. In all of these cases there can not exist a wandering domain.

Concerning the set of escaping points, that is, the set of points which tend to $\infty$ under iterates, there exists only two possibilities as stated in the corollary below. It particular it reflects the complexity of the dynamics for points in any neighbourhood of infinity.

Corollary 3.9. (Cor. 10) If $g_{\lambda}^{n}(z) \rightarrow \infty$, then $z \in \mathcal{B}_{\lambda} \cup \mathcal{J}\left(g_{\lambda}\right)$.

\subsection{Approximation of indifferent fixed points}

We showed in Section 2.1 that the approximating family $g_{\lambda, \mu}$ always has an attracting fixed point $z_{\mu}$ which converges to $-\infty$ as $\mu \rightarrow 0$. Furthermore we showed, that if the free critical point $c_{0}$ of the limit function $g_{\lambda}$ belongs to a basin of 
attraction for a given $\lambda_{0}$, then there exists a $\mu_{0}$ such that the function $g_{\lambda, \mu}$ also has an attracting periodic point for this parameter $\lambda_{0}$ and for every $\mu \leq \mu_{0}$; see Section 2.2 for explicit computations restricted to the real line.

A different approach to the convergence of fixed points can be made through the parameter plane, that is, by considering a parameter $\lambda_{0}$ for which $g_{\lambda_{0}}$ has certain dynamical properties and approximating the function by $g_{\lambda_{0}, \mu}$. Lauber showed that the parameter plane of the function $g_{\lambda}$ contains a copy of the Mandelbrot set $M_{\lambda}$, where the main cardioid corresponds to the parameters for which the critical point $c_{0}$ belongs to the basin of an attracting fixed point $z_{0}$. Our result on polynomial-like mappings presented in Section 2.5 implies that the parameter plane $\lambda$ of the function $g_{\lambda, \mu}$ also contains a copy of the Mandelbrot set, which we denote by $M_{\lambda, \mu}$. The persistence of attracting cycles under uniform convergence of functions implies that for a given parameter $\lambda_{0} \in M_{\lambda}$ the attracting fixed point $z_{0}$ can be approximated by a sequence of attracting fixed points $z_{0, \mu}$ of $g_{\lambda, \mu}$. In other words, given a fixed point $z_{0}$ with multiplier $\left|g_{\lambda}^{\prime}\left(z_{0}\right)\right|<1$, there exists a $\mu_{*}$ and a sequence of fixed points $z_{0, \mu}$ such that $\left|g_{\lambda, \mu}^{\prime}\left(z_{0}\right)\right|<1$ for every $\mu \leq \mu_{*}$. The latter arguments in turn imply that the basin of attraction of $z_{0}$ is approximated by the attracting basins of $z_{0, \mu}$.

Our present question is whether we can extend this result to an arbitrary but fixed parameter $\lambda$ lying on the boundary of the domain $M_{\lambda}$. We obtain a positive answer for parameters lying on the boundary of the main cardioid. To achieve this, we describe the indifferent fixed point of $g_{\lambda}$ denoted by $z_{p}$, by solving the fixed point equation $g_{\lambda}(z)=z$. Through this equation we express the parameter as $\lambda_{p}=\frac{e^{-z_{p}}}{z_{p}}$. For such parameters, $z_{p}$ is an indifferent fixed point if and only if $z_{p}$ lies on the boundary of the disc $D\left(-\frac{2}{3}, \frac{1}{3}\right)$. For the parameter $\lambda_{p}$ and the corresponding indifferent fixed point $z_{p}$ there exists a sequence of fixed points of $g_{\lambda, \mu}$, denoted by $z_{p, \mu}$, that converge to $z_{p}$ as $\mu \rightarrow 0$. Further below in Theorem 3.10 we prove that the points $z_{p, \mu}$ are attracting. In the proof of the theorem we approximate the multiplier $g_{\lambda, \mu}^{\prime}$ using a quotient function $Q\left(z_{p}\right)$. Some properties of $Q\left(z_{p}\right)$ imply that the restriction of the parameter $\mu$ to $\mathbb{R}$ is convenient as we discuss in Remark 3.1.

In the present section we firstly describe the indifferent fixed points $z_{p}$ of $g_{\lambda}$ and their approximation by attracting fixed points $z_{p, \mu}$ of $g_{\lambda, \mu}$. This is proved in Theorem 3.10. Later on we analyse the quotient function $Q\left(z_{p}\right)$ and approximate the points $z_{p, \mu}$ by means of the Implicit Function Theorem and the points $z_{p}$.

The fixed point equation of the function $g_{\lambda}$ provides an expression of the parameter with respect to the fixed point, that is, by solving $g_{\lambda}(z)=z-1+\lambda z e^{z}$ equal to 
$z$. Then we can write the parameters that satisfy the equation

$$
\lambda=\frac{e^{-z}}{z} .
$$

The substitution of $\lambda$ in the derivative yields $g_{\lambda}^{\prime}(z)=1+\lambda e^{z}(z+1)=2+\frac{1}{z}$, valid for every fixed point. Looking for indifferent fixed points the equation

$$
\left|g_{\lambda}^{\prime}\left(z_{p}\right)\right|=\left|2+\frac{1}{z_{p}}\right|=1
$$

describes the points $z_{p}$ on the boundary of the disc with centre $-\frac{2}{3}$ and radius $\frac{1}{3}$. Solving the equation above we parametrise the points $z_{p}=x_{p}+i y_{p}$ as

$$
y_{p}^{2}=\frac{-3 x_{p}^{2}-4 x_{p}-1}{3} .
$$

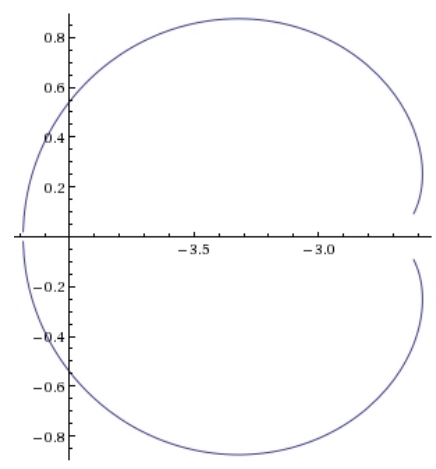

Figure 3.1: Curve $\mathcal{P}=\left\{\lambda_{p} \in \mathbb{C}^{*}: \lambda_{p}\left(z_{p}\right)=\frac{e^{-z_{p}}}{z_{p}}\right\}$ describing the parameters $\lambda_{p}$ for which the function $g_{\lambda}(z)=z-1+\lambda z e^{z}$ has an indifferent fixed point $z_{p} \in \partial D\left(-\frac{2}{3}, \frac{1}{3}\right)$. The discontinuity on the real axis is due to the singularity of the parametrisation for $z_{p}=-1$ at $\lambda_{p}=-e$ and numerical computations.

We describe in the parameter plane a curve of parameters $\lambda_{p}$ as a function of the indifferent fixed points $z_{p}$ using the parametrisation of the points $z_{p}$. The curve

$$
\mathcal{P}:=\left\{\lambda_{p} \in \mathbb{C}^{*}: \lambda_{p}\left(z_{p}\right)=\frac{e^{-z_{p}}}{z_{p}}\right\} \quad \text { with } \quad z_{p} \in \partial D\left(-\frac{2}{3}, \frac{1}{3}\right)
$$

has a cardioid form. Seen as a function, $\lambda_{p}\left(z_{p}\right)$ has a critical point at $z_{p}=-1$, since $\lambda_{p}^{\prime}\left(z_{p}\right)=\frac{-e^{z_{p}}\left(z_{p}+1\right)}{z_{p}}$. The curve meets the real axis at $\lambda_{p}(-1)=-e$ and at $\lambda_{p}\left(-\frac{1}{3}\right)=-3 e^{1 / 3} ;$ see Figure 3.1 . 
Fix a parameter $\lambda_{p} \neq-e$ on the curve $\mathcal{P}$ and let $z_{p} \neq-1$. The fixed point equation for the function $g_{\lambda_{p}, \mu}(z)=(1-\mu)\left(z-1+\frac{z}{z_{p}} e^{z-z_{p}}\right)$ has a solution if and only if

$$
\frac{e^{z-z_{p}}}{z_{p}}-\frac{1}{z}=-1+\frac{1}{1-\mu}
$$

Denote by $z_{p, \mu}$ the points $z$ that solve Equation 3.4 for given $\mu \in\left(0, \frac{1}{2}\right)$. It is clear that the points $z_{p, \mu}$ converge to $z_{p}$ as $\mu$ tends to zero. We claim that the points $z_{p, \mu}$ lie on a curve slightly shifted to the right from the disc $D\left(-\frac{2}{3}, \frac{1}{3}\right)$; see Figure 3.2. Moreover, we prove in Theorem 3.10 that the points $z_{p, \mu}$ are attracting fixed points for the function $g_{\lambda, \mu}$.

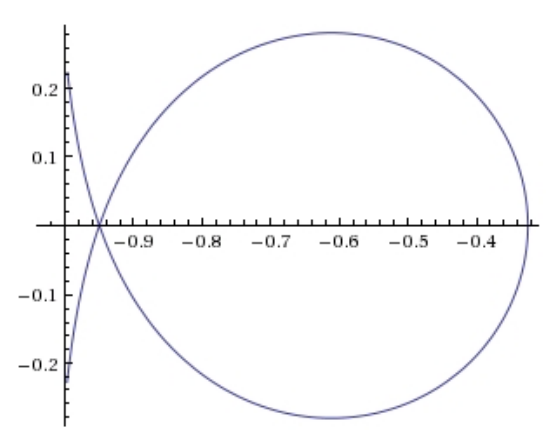

Figure 3.2: Curve containing the points $z_{p, \mu}$ for $\mu=\frac{1}{20}$ given through the approximation $z_{p, \mu} \simeq z_{p}+\mu \frac{z_{p}^{2}}{z_{p}+1}$. Recall that $z_{p, \mu}$ tends to $z_{p} \in$ $\partial D\left(-\frac{2}{3}, \frac{1}{3}\right)$ as $\mu \rightarrow 0$.

Theorem 3.10. Let $\lambda_{p} \in \mathcal{P}$ be a fixed parameter with $z_{p} \in \partial D\left(-\frac{2}{3}, \frac{1}{3}\right)$ and $z_{p} \neq-1$. Then for every $z_{p}$ it follows $z_{p, \mu} \rightarrow z_{p}$ as $\mu \rightarrow 0$. Furthermore there exists a sufficiently small $\mu_{0}$ such that the fixed points $z_{p, \mu}$ are attracting with respect to the function $g_{\lambda, \mu}$ for every $\mu \leq \mu_{0}$.

Proof. The points $z_{p, \mu}$ are defined as the solutions of the fixed point equation $g_{\lambda, \mu}(z)-z=0$, regarding the functions $g_{\lambda, \mu}$ as a perturbation of $g_{\lambda}$ with respect to $\mu$. Even though we cannot compute these solutions explicitly, we obtain an approximation for the points $z_{p, \mu}$ through its limit value $z_{p}$. We show in Lemma 3.12 below that $z_{p, \mu} \simeq z_{p}+\mu\left(\frac{z_{p}^{2}}{z_{p}+1}\right)$. Using this expression in the derivative $g_{\lambda, \mu}^{\prime}$ 
we obtain:

$$
\begin{aligned}
g_{\lambda_{p}, \mu}^{\prime}\left(z_{p, \mu}\right) & =(1-\mu)\left(1+\frac{e^{z_{p, \mu}-z_{p}}}{z_{p}}\left(z_{p, \mu}+1\right)\right) \\
& \simeq(1-\mu)\left(1+e^{\left(\mu \frac{z_{p}^{2}}{z_{p}+1}\right)}\left(\frac{1}{z_{p}}\right)\left(z_{p}+\mu \frac{z_{p}^{2}}{z_{p}+1}+1\right)\right) \\
& \simeq(1-\mu)\left(1+\left(1+\mu \frac{z_{p}^{2}}{z_{p}+1}\right)\left(\frac{z_{p}+1}{z_{p}}+\frac{\mu z_{p}}{z_{p}+1}\right)\right)
\end{aligned}
$$

where the last approximation is made by means of Taylor expansion, since $e^{\mu \frac{z_{p}^{2}}{z_{p}+1}} \simeq$ $\left(1+\mu \frac{z_{p}^{2}}{z_{p}+1}\right)$ for small values of $\mu$. Proceeding forward with the computations and associating terms with respect to $\mu$, we obtain

$$
\begin{aligned}
g_{\lambda_{p}, \mu}^{\prime}\left(z_{p, \mu}\right) \simeq 2 & +\frac{1}{z_{p}}+\mu\left(-1-\frac{1}{z_{p}}-\frac{1}{z_{p}+1}+z_{p}\right) \\
& +\mu^{2}\left(-1+\frac{1}{z_{p}+1}+\frac{z_{p}^{3}}{\left(z_{p}+1\right)^{2}}\right)+\mu^{3}\left(\frac{-z_{p}^{3}}{\left(z_{p}+1\right)^{2}}\right) \\
\simeq & \left(2+\frac{1}{z_{p}}\right)\left(1+\mu \cdot Q\left(z_{p}\right)\right)
\end{aligned}
$$

neglecting the terms of order two and three. The function $Q\left(z_{p}\right)$ denotes the quotient

$$
Q\left(z_{p}\right):=\left(-1-\frac{1}{z_{p}}-\frac{1}{z_{p}+1}+z_{p}\right) \cdot\left(2+\frac{1}{z_{p}}\right)^{-1} .
$$

We claim that $\left|g_{\lambda, \mu}^{\prime}\left(z_{p, \mu}\right)\right|<1$. An analysis of both the real and imaginary parts of $Q\left(z_{p}\right)$ shown below in Lemma 3.11 implies the existence of a sufficiently small $\mu_{0}$, with $\mu_{0}=\mu_{0}\left(\varepsilon_{1}, \varepsilon_{2}, z_{p}\right)$, such that using Equation (3.6) we obtain

$$
\begin{aligned}
\left|g_{\lambda, \mu}^{\prime}\left(z_{p, \mu}\right)\right|^{2} & \simeq\left|2+\frac{1}{z_{p}}\right|^{2}\left|1+\mu \cdot Q\left(z_{p}\right)\right|^{2}=\left|1+\mu \cdot Q\left(z_{p}\right)\right|^{2} \\
& =\left(1+\mu \operatorname{Re} Q\left(z_{p}\right)\right)^{2}+\left(\mu \operatorname{Im} Q\left(z_{p}\right)\right)^{2} \\
& <\left(1-\varepsilon_{1}\right)^{2}+\left(\varepsilon_{2}\right)^{2}<1
\end{aligned}
$$

for every $z_{p} \in \partial D\left(-\frac{2}{3}, \frac{1}{3}\right) \backslash\{-1\}$ and for some appropriate positive $\varepsilon_{1}$ and $\varepsilon_{2}$.

Note that the value $\varepsilon_{2}$ strongly depends on the point $z_{p}$ since its imaginary part $\left|\operatorname{Im} Q\left(z_{p}\right)\right|$ tends to $\infty$ as $x_{p} \rightarrow-1$, which is proved in Lemma 3.11 below. But for 
every fixed value $x_{p}>-1$ there exists a $\mu$ such that $\mu \operatorname{Im} Q\left(z_{p}\right)<\varepsilon_{2}$ as required. We present now the tools used in the proof of Theorem 3.10 and further results related to the parabolic fixed point and its approximation.

Lemma 3.11. The function $Q\left(z_{p}\right)=\left(-1-\frac{1}{z_{p}}-\frac{1}{z_{p}+1}+z_{p}\right) \cdot\left(2+\frac{1}{z_{p}}\right)^{-1}$ has negative real part for every $z_{p} \in \partial D\left(-\frac{2}{3}, \frac{1}{3}\right)$ and the imaginary part tends to $\pm \infty$ as $z_{p} \rightarrow-1$.

Proof. Using the parametrisation of the points $z_{p}=x_{p}+i y_{p}$ as described in Equation 3.3 for $x_{p} \in\left(-1,-\frac{1}{3}\right]$ we write the real and imaginary parts of $Q\left(z_{p}\right)$ as real functions of $x_{p}$ as follows

$$
\operatorname{Re} Q\left(z_{p}\right):=u\left(x_{p}\right)=\frac{4 x_{p}^{2}-26 x_{p}-9}{2\left(4 x_{p}+1\right)}
$$

and

$$
\operatorname{Im} Q\left(z_{p}\right):=v\left(x_{p}\right)= \pm \sqrt{\frac{\left(3 x_{p}+1\right)\left(-x_{p}-1\right)}{3}}\left(\frac{64 x_{p}^{3}+84 x_{p}^{2}-24 x_{p}-17}{2\left(4 x_{p}+1\right)^{2}\left(x_{p}+1\right)}\right) .
$$

By simple computations we obtain that $u\left(x_{p}\right)=0$ for $x_{1,2}=\frac{1}{4}(13 \pm \sqrt{205})$. It follows from

$$
u^{\prime}\left(x_{p}\right)=\frac{8 x_{p}^{2}+4 x_{p}+5}{\left(4 x_{p}+1\right)^{2}}>0 \quad \text { for every } \quad x_{p} \in \mathbb{R}
$$

that $u\left(x_{p}\right)<0$ for every $x_{p} \leq-\frac{1}{3}<x_{1} \approx-0.3294$. Furthermore $v\left(x_{p}\right)$ is only defined for values of $x_{p} \in\left(-1,-\frac{1}{3}\right]$. Furthermore, $v(x)$ tends to $\pm \infty$ as $x_{p} \rightarrow-1$ while it equals zero at $x_{1}=-\frac{1}{3}$ and $x_{2} \approx-0367697$.

Lemma 3.12. Let $z_{p}$ be an indifferent fixed point of $g_{\lambda}$, with $z_{p} \neq-1$. Then the approximating fixed points $z_{p, \mu}$ of $g_{\lambda, \mu}$ can be expressed as $z_{p, \mu} \simeq z_{p}+\mu\left(\frac{z_{p}^{2}}{z_{p}+1}\right)$.

Proof. We analyse the points $z_{p, \mu}$ as they converge to the indifferent fixed points $z_{p}$ on the boundary of the disc $D\left(-\frac{2}{3}, \frac{1}{3}\right)$. This approximation can be seen as a continuous function of $\mu$ representing the perturbation of $g_{\lambda}$ by the factor $1-\mu$. Therefore, consider during this proof the function $g_{z_{p}}(\mu): \mathbb{C} \rightarrow \mathbb{C}$ with $\mu \mapsto$ $(1-\mu)\left(z-1+\frac{z}{z_{p}} e^{z-z_{p}}\right)$ with a complex parameter $\mu$. Then using the Implicit Function Theorem, if the function

$$
G(\mu, z)=(1-\mu)\left(z-1+\frac{z}{z_{p}} e^{z-z_{p}}\right)-z
$$




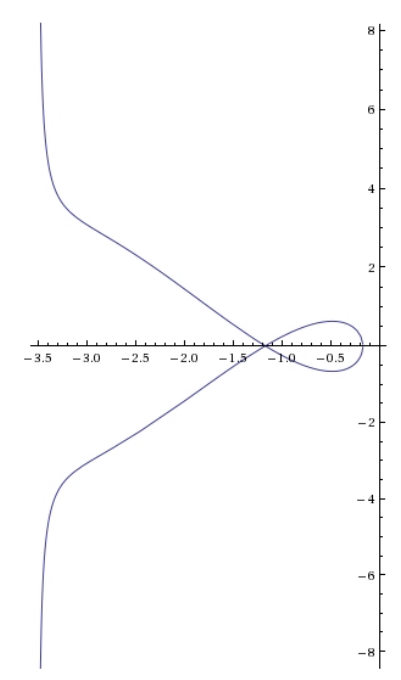

Figure 3.3: Graph of the quotient $Q\left(z_{p}\right)$ defined in Equation 3.7. The curve is parametrised for points $z_{p}=x_{p}+i y_{p}$ with $y_{p}^{2}=\left(-3 x_{p}^{2}-4 x_{p}-1\right) / 3$ and $x_{p} \in\left(-1,-\frac{1}{3}\right]$.

satisfies the equation $G\left(\mu_{0}, z_{0}\right)=0$ for some $\mu_{0}$ and $z_{0}$ and $\frac{\partial G}{\partial z}\left(\mu_{0}, z_{0}\right)$ is invertible, then there exist open neighbourhoods $U$ of $\mu_{0}$ and $V$ of $z_{0}$ as well as a unique holomorphic function $\widetilde{g}: U \rightarrow V$ such that

$$
\begin{gathered}
G(\mu, z)=G(\mu, \widetilde{g}(\mu)) \\
\frac{d \widetilde{g}}{d \mu}(\mu)=-\left(\frac{\partial G}{\partial z}(\mu, \widetilde{g}(\mu))\right)^{-1} \cdot \frac{\partial G}{\partial \mu}(\mu, \widetilde{g}(\mu))
\end{gathered}
$$

and $\widetilde{g}(\mu)=z$ holds for every $\mu \in U$ and $z \in V$. For our purpose consider the point $\left(\mu_{0}, z_{0}\right)=\left(0, z_{p}\right)$ for which $\frac{\partial G}{\partial z}(\mu, z)=(1-\mu)\left(1+\frac{e^{z-z_{p}}}{z_{p}}(z+1)\right)-1$ is invertible if and only if $z_{p} \neq-1$.

In particular, the solutions of $\widetilde{g}(\mu)=z$ coincide with $z_{p, \mu}$ for every $z \in V$. Even though the explicit expression $\widetilde{g}(\mu)$ only exists for $\mu_{0}=0$ and $z_{0}=z_{p}$, we can use the result to express $z_{p, \mu}=\widetilde{g}(\mu)$ by means of its Taylor expansion as

$$
z_{p, \mu}=\widetilde{g}\left(\mu_{0}\right)+\left(\mu-\mu_{0}\right) \frac{d \widetilde{g}}{d \mu}\left(\mu_{0}\right)+\mathcal{O}\left(\mu^{2}\right)
$$


for $\mu \in U$. By simple computations we obtain for $\mu_{0}=0$ and $z_{0}=z_{p}$

$$
\begin{aligned}
\frac{d \widetilde{g}}{d \mu}(0) & =-\left(\frac{\partial G}{\partial z}\left(0, z_{p}\right)\right)^{-1} \cdot \frac{\partial G}{\partial \mu}\left(0, z_{p}\right) \\
& =\left(1+\frac{e^{z_{p}-z_{p}}}{z_{p}}\left(z_{p}+1\right)-1\right)^{-1} \cdot\left(z_{p}-1+\frac{z_{p}}{z_{p}} e^{z_{p}-z_{p}}\right)=\frac{z_{p}^{2}}{z_{p}+1},
\end{aligned}
$$

obtaining the desired approximation for $z_{p, \mu}$ by neglecting the higher terms $\mathcal{O}\left(\mu^{2}\right)$.

Remark 3.1. Note that the approximation of the attracting fixed points $z_{p, \mu}$ depends strongly on the parameter $\mu$. Even though we consider $\mu$ as a complex parameter in the proof of Lemma 3.12, we take the restriction of $\mu$ as a real number. The proof of Theorem 3.10 requires that the norm of the multiplier $M_{\mu}=g_{\lambda, \mu}^{\prime}\left(z_{p, \mu}\right)$ is smaller than one. This in turn goes back to the quotient function $Q\left(z_{p}\right)$ analysed in Lemma 3.11. The condition that the norm $\left|1+\mu Q\left(z_{p}\right)\right|$ must be smaller than one would strongly restrict the values of $Q\left(z_{p}\right)$ if we consider $\mu$ as a complex number.

The reasoning of the remark is based on the computations of $\left|1+\mu Q\left(z_{p}\right)\right|$. Recall that $Q\left(z_{p}\right)$ is defined for $x \in\left(-1,-\frac{1}{3}\right]$ only. On the one hand, we see that $\operatorname{Im} Q\left(z_{p}\right)$ equals zero in this interval if and only if $x_{1}=-\frac{1}{3}$ and $x_{2} \approx-0.3676$. Furthermore, $\operatorname{Im} Q\left(z_{p}\right)$ has a local maximum (resp. local minimum for negative $\left.\operatorname{Im} Q\left(z_{p}\right)\right)$ at the point $x_{m} \approx-0.3417 \in\left(x_{2}, x_{1}\right)$. On the other hand, $\operatorname{Re} Q\left(z_{p}\right)$ takes approximated values -1.1692 at $x_{2}$ and -0.4809 at $x_{m}$. With this, for values of $x$ in $\left(x_{2}, x_{1}\right)$ we have $\left|Q\left(z_{p}\right)\right|<1.3670$. Hence for real $\mu<\frac{1}{2}$ it is satisfied that $\left|g_{\lambda_{p}, \mu}^{\prime}\left(z_{p, \mu}\right)\right| \simeq\left|1+\mu Q\left(z_{p}\right)\right|<1$. But as $x$ approaches -1 , values of the quotient $Q\left(z_{p}\right)$ have very large norm and its argument approaches $\pi / 2$ (resp. $-\pi / 2$ for $\left.\operatorname{Im} Q\left(z_{p}\right)<0\right)$. As we multiply with a complex parameter $\mu$, the real part of $\mu Q\left(z_{p}\right)$ equals $\operatorname{Re} \mu \cdot \operatorname{Re} Q\left(z_{p}\right)-\operatorname{Im} \mu \cdot \operatorname{Im} Q\left(z_{p}\right)$ which may be positive for $\mu$ with negative imaginary part. Hence in this case $\left(\operatorname{Re}\left(1+\mu Q\left(z_{p}\right)\right)\right)^{2}$ can easily be greater than one.

Recall that the value $x=-\frac{1}{3}$ corresponds to the indifferent fixed point $z_{p}=-\frac{1}{3}$ and the parameter $\lambda_{p}=-3 e^{1 / 3}$. The arguments above imply the existence of a (quite) small neighbourhood of $\lambda_{p}=-3 e^{1 / 3}$ where the approximation of the function of $g_{\lambda, \mu}$ with a complex parameter $\mu$ may be extended. But a general statement for all parameters $\lambda_{p}$ does not exists. It is of special difficulty to do the computations close to the parameter $\lambda_{p}=-e$ where the curve $\mathcal{P}$ has a cuspid.

The fact that $\frac{\partial G}{\partial z}\left(0, z_{p}\right)$ is not invertible at $z_{p}=-1$ agrees with the singularity of the curve $\mathcal{P}$ in the parameter plane. In conclusion, for $z_{p}=-1$ we can apply the 
Implicit Function Theorem to approximate $z_{p, \mu}$ through $z_{p}$, We can also not prove that these fixed points are attracting using the quotient function $Q\left(z_{0}\right)$. Hence we explicitly show in the proposition below the existence of a sequence of attracting fixed points converging to -1 . Within this proof the functions $g_{\lambda}$ and $g_{\lambda, \mu}$ are restricted to the real axis, since in this case the parameters and the fixed point are real, facilitating the proof.

Proposition 3.13. Let $\lambda_{p}=-e$. The function $g_{\lambda_{p}}$ has a parabolic fixed point at $z_{p}=-1$ with multiplier equals 1 . Furthermore, the points $z_{p, \mu}$ satisfying the equation $e^{z_{p, \mu}+1}+\frac{1}{z_{p, \mu}}=\frac{-\mu}{1-\mu}$ are attracting fixed points of the function $g_{\lambda_{p}, \mu}$ and converge to $z_{p}$ as $\mu$ tends to 0 .

Proof. During this proof we consider the function $g_{-e}(z)=z-1-z e^{z+1}$. The first part of the proposition follows by computing its derivative $g_{-e}^{\prime}(z)=1-e^{z+1}(z+1)$ at the point $z_{p}=-1$.

Consider now the functions $g_{-e, \mu}=(1-\mu)\left(z-1-z e^{z+1}\right)$, with $\mu \in\left(0, \frac{1}{2}\right)$. The fixed points of $g_{-e, \mu}$ are the solutions $z$ to the equation

$$
e^{z+1}+\frac{1}{z}=\frac{-\mu}{1-\mu}
$$

These solutions clearly converge to the fixed point of $g_{-e}$ as $\mu$ tends to zero, since $g_{-e}(z)=z$ if and only if $e^{z+1}=-\frac{1}{z}$. We have that $\frac{-\mu}{1-\mu}=1-\frac{1}{1-\mu}$ lies in the interval $\left(-\frac{1}{2}, 0\right)$ for $\mu \in\left(0, \frac{1}{2}\right)$. Since the parameter $\lambda_{p}=-e$ and the fixed point $z_{p}=-1$ are both real, we may restrict the functions $g_{-e, \mu}$ and $g_{-e}$ to the real axis for the rest of the proof. The function $f(x):=e^{x+1}+\frac{1}{x}$ takes values in $\left(-\frac{1}{2}, 0\right)$ for $x \in\left(-1,-\frac{1}{2}\right)$. Then we obtain from Equation 3.8

$$
1-\mu=\frac{1}{1-f(x)}=\frac{-x}{x e^{x+1}+1-x}
$$

and

$$
\begin{aligned}
g_{-e, \mu}^{\prime}(x) & =(1-\mu)\left(1-(x+1) e^{x+1}\right) \\
& =\frac{\left(x^{2}+x\right) e^{x+1}-x}{x e^{x+1}+1-x} .
\end{aligned}
$$

The function $g_{-e, \mu}^{\prime}(x)$ equals zero at $x_{1}=0$, at $x_{2} \approx-0.432$ and has the value $g_{-e, \mu}^{\prime}(-1)=1$. Furthermore the second derivative

$$
g_{-e, \mu}^{\prime \prime}(x)=\frac{x^{2} e^{2 x+2}+\left(-x^{3}+3 x+1\right) e^{x+1}-1}{x^{2} e^{2 x+2}+\left(-2 x^{2}-2 x\right) e^{x+1}+(1-x)^{2}}
$$


has zeros at $x_{1}^{\prime} \approx-6.5889, x_{2}^{\prime} \approx-1.6363$ and $x_{3}^{\prime} \approx-0.2191$. The point $x_{2}^{\prime}$ is a local maximum while $x_{3}^{\prime}$ is a local minimum with $h\left(x_{3}^{\prime}\right) \approx-0.2085$, as shown in Figure 3.4. Then the function $g_{-e, \mu}^{\prime}(x)$ has values in $(-0.2085,1)$ for $x \in(-1,0)$, implying that $\left|g_{\lambda, \mu}^{\prime}(x)\right|<1$ for every $x \in\left(-1,-\frac{1}{2}\right)$ as expected.

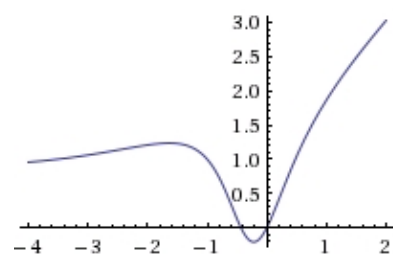

Figure 3.4: Graph of the function $g_{-e, \mu}^{\prime}(x)$ considered as a real function.

\subsection{Kernel convergence on the Fatou set}

In the previous chapter we described the dynamical behaviour of the family of functions $g_{\lambda, \mu}$. In Section 3.1 we introduced the limit family of functions $g_{\lambda}$ giving some results related to the Baker domain $\mathcal{B}_{\lambda}$ and wandering domains. Now we continue with the approximation of $\mathcal{B}_{\lambda}$ through the basins of attraction $\mathcal{A}_{\mu}$ of $g_{\lambda, \mu}$ as $\mu$ tends to zero. This is followed by the convergence to the parabolic basins $\mathcal{D}_{p}$ by attracting basins $\mathcal{A}_{p, \mu}$ for parameters $\lambda$ such that $g_{\lambda}$ has a parabolic fixed point. The first case automatically implies the kernel convergence of Fatou sets when $\mathcal{F}\left(g_{\lambda}\right)$ equals the Baker domain.

Recall that a domain $B$ is a kernel of a sequence of domains $\left\{B_{j}\right\}_{j \in I}$ if $B$ is the largest domain containing the marked point $z_{0}$ and such that for every compact set $K \subset \mathcal{B}$, implies that $K \subset B_{j}$ for all $B_{j}$ except at most finitely many. The convergence holds when $B$ is a kernel of every subsequence of $\left\{B_{j}\right\}$.

Note that Definitions 1.1 and 1.2 presented in Section 1.9 consider discrete sequences of open sets $B_{j}$ with $j$ in an index set $I$. In our case, the open sets depend on a continuous parameter $\mu \in\left(0, \frac{1}{2}\right)$. Therefore we may choose during the proofs an arbitrary subsequence of parameters $\left\{\mu_{j}\right\}_{j \in \mathbb{N}} \subset\left(0, \frac{1}{2}\right)$, with $\mu_{j} \rightarrow 0$ as $n \rightarrow \infty$. However, the arguments do not depend on the choice of $\left\{\mu_{j}\right\}_{j \in \mathbb{N}}$ but on the upper boundary value $\mu_{0}$ only. Hence we omit the subindex $j$ in the notation and present the results for arbitrary parameters $\mu \leq \mu_{0}$.

At first we present the approximation of the Baker domain $\mathcal{B}_{\lambda}$ by attracting basins $\mathcal{A}_{\mu}$ in Subsection 3.3.1. This is followed by the convergence of the attracting basins 
$\mathcal{A}_{p, \mu}$ to the parabolic basins $\mathcal{D}_{p}$ in Subsection 3.3 .2 . To this end, we firstly prove in Proposition 3.19 the convergence of the multipliers $M_{\mu}$ to $M_{0}$ of the points $z_{p, \mu}$ and $z_{p}$ respectively. In both cases we use the existence of the attracting fixed points $z_{\mu}$ and $z_{p, \mu}$ of the function $g_{\lambda, \mu}$ proved in previous sections.

\subsubsection{Kernel convergence to the Baker domain $\mathcal{B}_{\lambda}$}

In Section 2.4 we constructed an invariant absorbing domain $\mathcal{H}_{\eta, \rho}$ for the attracting basin $\mathcal{A}_{\mu}$ and introduced the analogue absorbing domain $\mathcal{M}_{\delta}$ for the Baker domain $\mathcal{B}_{\lambda}$. Now we prove the convergence of $\mathcal{H}_{\eta, \rho}$ to $\mathcal{M}_{\delta}$ as kernel in the sense of Carathéodory.

For the function $g_{\lambda}$ the absorbing domain $\mathcal{M}_{\delta}$ is defined as the set of points $z \in \mathbb{C}$ for which their image under $g_{\lambda}$ is at a distance $\delta$ from $z-1$, with $\delta \in(0,1)$. However we could assured the invariance of the domain $\mathcal{H}_{\eta, \rho}$ for $\delta=\frac{1}{2}$; see Subsection 2.4.2. Hence we take the fixed value $\delta=1 / 2$ also for $\mathcal{M}_{\delta}$ obtaining:

$$
\begin{gathered}
\mathcal{M}_{1 / 2}=\left\{z \in \mathbb{C}:\left|g_{\lambda}(z)-(z-1)\right|<\frac{1}{2}\right\}=\left\{x+i y: y^{2}<\rho(x)\right\} \\
\text { for } \rho(x):=\frac{e^{-2 x}}{(2|\lambda|)^{2}}-x^{2}
\end{gathered}
$$

The absorbing domain $\mathcal{H}_{\eta, \rho}$ invariant under the function $g_{\lambda, \mu}$ is defined as the union of two smaller domains for $\delta=\frac{1}{2}$ and $\mu \leq \mu_{0}, \mu_{0} \in\left(0, \frac{1}{2}\right)$, as follows

$$
\begin{gathered}
\mathcal{H}_{\eta, \rho}=\mathcal{H}_{\eta} \cup \mathcal{M}_{\rho}, \quad \text { with } \\
\mathcal{H}_{\eta}:=\left\{z=x+i y: x \leq 1-\frac{1}{2 \mu} \text { and }|y|<\eta_{\mu}(x)\right\} \\
\text { for } \eta_{\mu}(x):=-x \sqrt{\left(\frac{\mu e^{(1-2 \mu) / 2 \mu}}{(2 \mu-1)(1-\mu)|\lambda|}\right)^{2}-1},
\end{gathered}
$$

and

$$
\begin{gathered}
\mathcal{M}_{\rho}:=\left\{z=x+i y: 1-\frac{1}{2 \mu}<x<x_{0} \quad \text { and } \quad y^{2}<\rho_{\mu}(x)\right\} \\
\text { for } \quad \rho_{\mu}(x)=\left(\frac{e^{-x}}{2(1-\mu)|\lambda|}\right)^{2}-x^{2} \quad \text { and } \quad \rho\left(x_{0}\right) \geq 0
\end{gathered}
$$


The boundary value $\mu_{0}$ for the parameter $\mu$ is chosen sufficiently small such that $|\lambda|<e^{\frac{1}{2}\left(\frac{1}{\mu}-2\right)} \frac{\mu^{2}}{1-\mu}$ holds for all $\mu \leq \mu_{0}$, which guarantees the existence of the attracting fixed point $z_{\mu}$ and the invariance of the absorbing domain.

Comparing Equations (3.9) and (3.12) we can see that as $\mu \rightarrow 0$, the parametrisation $\rho_{\mu}(x)$ uniformly converges to $\rho(x)$ as real functions. Furthermore, it is also true that the graph of $\rho_{\mu}$ converges to the graph of $\rho$ as compact subsets of $\widehat{\mathbb{C}}$ in the Hausdorff metric; see Figure 3.5. Hence the functions $\pm \sqrt{\rho(x)}$ and $\pm \sqrt{\rho(x)}$ satisfy the same properties. We remark that these arguments apply to the boundaries of $\mathcal{M}_{1 / 2}$ and $\mathcal{M}_{\rho}$ but not necessarily for the entire $\partial \mathcal{H}_{\eta, \rho}$. Nevertheless, we consider $\mu$ sufficiently small that $1-\frac{1}{2 \mu}$ is large negative, in addition to the properties mentioned above, so that the domain $\mathcal{M}_{\rho}$ is sufficiently large for our purposes.

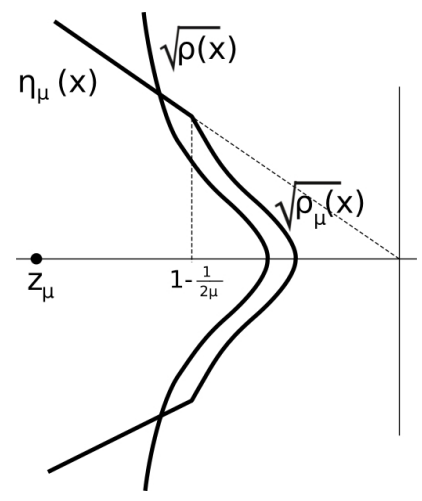

Figure 3.5: Boundaries of absorbing domains $\partial \mathcal{M}_{1 / 2}=\left\{x+i y: y^{2}=\right.$ $\rho(x)\}$ and $\partial \mathcal{H}_{\eta, \rho}=\left\{x+i y: x>1-\frac{1}{2 \mu} ; y^{2}=\rho_{\mu}(x)\right\} \cup\{x+i y: x \leq$ $\left.1-\frac{1}{2 \mu}, y= \pm \eta_{\mu}(x)\right\}$.

We now present the kernel convergence of the absorbing domains. This is followed by Lemma 3.16 showing the persistence of compact sets useful in the convergence proof. Afterwards we present Lemma 3.15 showing some properties of the functions $\rho_{\mu}(x)$ and $\eta_{\mu}(x)$. Lemma 3.15 implies in particular that the domains $\mathcal{H}_{\eta, \rho}$ do not form a nested sequence of sets, which would immediately imply the kernel convergence of $\mathcal{H}_{\eta, \rho}$ to the domain $\mathcal{M}_{1 / 2}$; compare with the discussion on this topic in Section 1.9 .

Theorem 3.14. Let $\mathcal{M}_{1 / 2}$ and $\mathcal{H}_{\eta, \rho}$ be absorbing domains for $\mathcal{B}_{\lambda}$ under $g_{\lambda}$ and for $\mathcal{A}_{\mu}$ under $g_{\lambda, \mu}$ respectively as defined in Equations (3.9) and (3.10). Then $\mathcal{H}_{\eta, \rho}$ converges to $\mathcal{M}_{1 / 2}$ as kernel in the sense of Carathéodory as $\mu \rightarrow 0$. 
Proof. Let $\lambda \in \mathbb{C}^{*}$ and $\mu_{0} \in\left(0, \frac{1}{2}\right)$ both be arbitrary but fixed parameters, with $\mu_{0}$ sufficiently small that the absorbing domain $\mathcal{H}_{\eta, \rho}$ is invariant. In order to make clear the dependence of the domains $\mathcal{H}_{\eta, \rho}$ on the parameter $\mu$ during this proof, we specify it in the notation as $\mathcal{H}_{\eta, \rho}^{\mu}$ or $\mathcal{H}_{\eta}^{\mu}$ and $\mathcal{M}_{\rho}^{\mu}$ for the subdomains. We start by mentioning some properties of the boundary curves. Then we give an appropriate marked point, prove the compact set property and finish by proving the validity for every subsequence.

Making some computations we can see that

$$
0<\rho(x)=\frac{e^{-2 x}}{(2|\lambda|)^{2}}-x^{2}<\left(\frac{1}{1-\mu}\right)^{2} \frac{e^{-2 x}}{(2|\lambda|)^{2}}-x^{2}=\rho_{\mu}(x),
$$

for every $\mu<\frac{1}{2}$ and $x \in \mathbb{R}$. Hence it is sufficient to take a value $x_{0}$ such that $\rho(x) \geq 0$ for all $x \leq x_{0}$, implying that $\rho_{\mu}(x)$ is also well defined for every $x \leq x_{0}$ and every $\mu$. We obtain that

$$
\rho_{\mu}(x)<\rho_{\mu_{0}}(x) \text { and } \eta_{\mu_{0}}(x)<\eta_{\mu}(x)
$$

for every $0<\mu<\mu_{0}$ as we show below in Lemma 3.15 .

We firstly show that $\mathcal{M}_{1 / 2}$ contains the marked point of $H_{\eta, \rho}^{\mu}$ for every $\mu \in\left(0, \frac{1}{2}\right)$. As marked point we use the attracting fixed point $z_{\mu_{0}}$ (depending on the fixed parameter $\left.\mu_{0}\right)$ and which lies in the interval $\left(-\frac{1}{\mu_{0}}, 2-\frac{1}{\mu_{0}}\right)$ as it is proved in Corollary 2.2. We claim this holds by construction that $z_{\mu_{0}} \in \mathcal{H}_{\eta, \rho}^{\mu}=\mathcal{H}_{\eta}^{\mu} \cup \mathcal{M}_{\rho}^{\mu}$ for every $\mu \in\left(0, \frac{1}{2}\right)$. On the one hand, if there exists a $\mu_{*}$ such that $z_{\mu_{0}}>1-\frac{1}{2 \mu_{*}}$, that is $z_{\mu_{0}} \in \mathcal{M}_{\rho}^{\mu_{*}}$, then it implies $z_{\mu_{0}} \in \mathcal{M}_{\rho}^{\mu}$ for all $\mu<\mu_{*}$, since $\rho_{\mu}(x)<\rho_{\mu_{*}}(x)$ and $1-\frac{1}{2 \mu_{*}}<1-\frac{1}{2 \mu}$ for such parameters $\mu$. On the other hand, if for some $\mu_{*}$ it holds $z_{\mu_{0}} \leq 1-\frac{1}{2 \mu_{*}}$, that is $z_{\mu_{0}} \in \mathcal{H}_{\eta}^{\mu_{*}}$, then $z_{\mu_{0}} \in \mathcal{H}_{\eta}^{\mu}$ for all $\mu \geq \mu_{*}$. In particular, it follows that $z_{\mu_{0}}<x_{0}<\sigma_{\mu}:=\min \left\{-e, 2 \ln \left(\frac{1}{2(1-\mu)|\lambda|}\right)\right\}$; compare with Equation (3.13) and Proposition 2.28 for the construction of $\sigma_{\mu}$. Hence $z_{\mu_{0}}< \pm \sqrt{\rho(x)}$ and lies in $\mathcal{M}_{1 / 2}$ as desired.

We continue by showing that given any compact set $K \subset \mathcal{M}_{1 / 2}$, there exists a $\mu_{*}$ such that $K$ is also contained in $\mathcal{H}_{\eta, \rho}^{\mu}$ for every $\mu \leq \mu_{*}$. Let $\mu_{*}$ be an arbitrary but fixed parameter. If $K \subset \mathcal{M}_{1 / 2} \cap\left\{z \in \mathbb{C}: \operatorname{Re} z>1-\frac{1}{2 \mu_{*}}\right\}$ then the result immediately follows from Inequality $(3.13)$. Hence we may assume that $K \cap\left\{z \in \mathbb{C}: \operatorname{Re} z \leq 1-\frac{1}{2 \mu_{0}}\right\} \neq \emptyset$ and denote the intersection by $\widetilde{K}$. Since $\widetilde{K}$ is bounded and $\eta_{\mu}(x)$ approaches $\sqrt{\rho(x)}$ from below (resp. $-\eta_{\mu}(x)$ approaches $-\sqrt{\rho(x)}$ from above), we claim that for every $z \in \widetilde{K}$ there exists $\mu_{*}$ such that $|\operatorname{Im} z|<\eta_{\mu_{*}}(\operatorname{Re} z)$ and with this $|\operatorname{Im} z|<\eta_{\mu}(\operatorname{Re} z)$ for every $\mu \leq \mu_{*} ;$ compare with 
Inequation (3.14). If this is not the case, we may assume that $\operatorname{Re} z>1-\frac{1}{2 \mu_{*}}$ which leads to the first assumption. Otherwise we can always choose a smaller $\mu_{*}$ such that every $z \in K$ satisfies one of the both cases mentioned above, that is, either $\widetilde{K}=\emptyset$ and $z \in \mathcal{M}_{\rho}^{\mu *}$ or $z \in \mathcal{H}_{\eta}^{\mu *}$.

To prove that $\mathcal{M}_{1 / 2}$ is kernel for every subsequence $\left\{\mathcal{H}_{\eta, \rho}^{\mu}\right\}_{\mu_{j}}$ is equivalent to prove that there exists a $\mu_{*}$ such that if $U$ is an open subset of $\mathcal{H}_{\eta, \rho}^{\mu}$ for every $\mu \leq \mu_{*}$, then $U \subset \mathcal{M}_{1 / 2}$ as well. For every $z \in U$ it holds $z \in \mathcal{H}_{\eta, \rho}^{\mu}=\mathcal{H}_{\eta}^{\mu} \cup \mathcal{M}_{\rho}^{\mu}$. Then either $z$ is contained in $\mathcal{H}_{\eta}^{\mu}$ for every $\mu$ or there exists a $\mu_{*}$ such that $1-\frac{1}{2 \mu_{*}}<\operatorname{Re} z$ for every $\mu \leq \mu_{*}$. In the first case, the proof follows immediately. In the latter case it holds that $z \in \mathcal{M}_{\rho}^{\mu}$ for all $\mu \leq \mu_{*}$. Assume $z \notin \mathcal{M}_{1 / 2}$. This implies that $|\operatorname{Im} z|>\rho(\operatorname{Re} z)$. Since $U$ is an open set, there exists $\varepsilon>0$ such that $\operatorname{dist}(z, \rho(x)) \geq \varepsilon, D(z, \varepsilon / 2) \subset U$ and $D(z, \varepsilon / 2) \subset \mathbb{C} \backslash \mathcal{M}_{1 / 2}$. Since $\rho_{\mu} \rightarrow \rho$ in the Hausdorff metric, there exists a $\widetilde{\mu}$ such that $\operatorname{dist}_{H}\left(\rho, \rho_{\mu}\right)<\varepsilon / 2$ for every $\mu<\widetilde{\mu}$. In particular, this implies also that $|\operatorname{Im} z| \geq \rho_{\mu}(\operatorname{Re} z)$ for every $\mu<\widetilde{\mu}$ and with this $z \notin \mathcal{M}_{\rho}^{\mu}$ leading to a contradiction.

The convergence of $\rho_{\mu}$ to $\rho$ implies that $\mathcal{M}_{1 / 2}$ is a maximal set satisfying the properties of a kernel. Finally we assume without loss of generality that $\mu_{*} \leq \mu_{0}$, otherwise we may take a smaller value for $\mu_{*}$ without changing the arguments above.

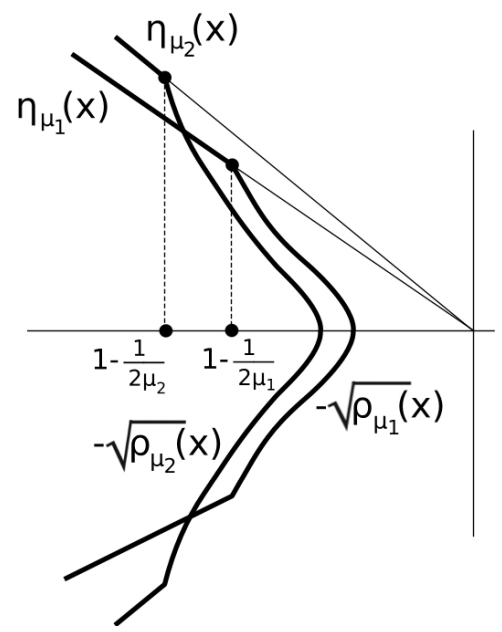

Figure 3.6: Boundaries of absorbing domains $\mathcal{H}_{\eta, \rho}$ for two different parameters $\mu_{2}<\mu_{1}<\frac{1}{2}$. The figure illustrates that the absorbing domains do not form a nested sequence of domains as $\mu$ tends to zero.

Now we present some of the properties of the curves $\rho_{\mu}$ and $\eta_{\mu}$ used in the proof 
above. This properties depend on the parameter $\mu$, only, and are independent of the parameter $\lambda$.

Lemma 3.15. Let $x$ be a negative real number having an arbitrary but fixed value and $0<\mu_{0}<1 / 2$ sufficiently small such that the invariance of the absorbing domain $\mathcal{H}_{\eta, \rho}=\mathcal{H}_{\eta} \cup \mathcal{M}_{\rho}$ is guaranteed. Then for parameters $\mu_{a}<\mu_{b} \leq \mu_{0}$ we obtain:

- The parametrisations of $\partial \mathcal{M}_{\rho}^{\mu_{a}}$ and $\partial \mathcal{M}_{\rho}^{\mu_{b}}$ satisfy $\left|\sqrt{\rho_{\mu_{a}}(x)}\right|<\left|\sqrt{\rho_{\mu_{b}}(x)}\right|$.

- The parametrisations of $\partial \mathcal{H}_{\eta}^{\mu_{a}}$ and $\partial \mathcal{H}_{\eta}^{\mu_{b}}$ satisfy $\left|\eta_{\mu_{a}}(x)\right|>\left|\eta_{\mu_{b}}(x)\right|$.

Proof. We prove the statements for the positive values of parametrisations $\rho_{\mu}(x)$ and $\eta_{\mu}(x)$ and they analogously follow for the negative values. The first statement follows from the definition $\rho_{\mu}(x)=\left(\frac{1}{1-\mu}\right)^{2} \frac{e^{-2 x}}{(2|\lambda|)^{2}}-x^{2}$, since $1<\frac{1}{1-\mu_{a}}<\frac{1}{1-\mu_{b}} \leq$ $\frac{1}{1-\mu_{0}}$ for all $\mu_{a}<\mu_{b} \leq \mu_{0}<1 / 2$.

To prove the second statement we obtain for $x<0$ that $\eta_{\mu_{b}}(x)<\eta_{\mu_{a}}(x)$ if and only if

$$
\begin{array}{rlrl} 
& -x \sqrt{\left(\frac{\mu_{b} e^{\left(1-2 \mu_{b}\right) / 2 \mu_{b}}}{\left(2 \mu_{b}-1\right)\left(1-\mu_{b}\right)|\lambda|}\right)^{2}-1} & <-x \sqrt{\left(\frac{\mu_{a} e^{\left(1-2 \mu_{a}\right) / 2 \mu_{a}}}{\left(2 \mu_{a}-1\right)\left(1-\mu_{a}\right)|\lambda|}\right)^{2}-1} \\
\left|\frac{\mu_{b} e^{\left(1-2 \mu_{b}\right) / 2 \mu_{b}}}{\left(2 \mu_{b}-1\right)\left(1-\mu_{b}\right)}\right| & <\left|\frac{\mu_{a} e^{\left(1-2 \mu_{a}\right) / 2 \mu_{a}}}{\left(2 \mu_{a}-1\right)\left(1-\mu_{a}\right)}\right| \\
e^{\frac{1}{2}\left(\frac{1}{\mu_{b}}-\frac{1}{\mu_{a}}\right)} & <\frac{\mu_{a}}{\left(1-2 \mu_{a}\right)\left(1-\mu_{a}\right)} \frac{\left(1-2 \mu_{b}\right)\left(1-\mu_{b}\right)}{\mu_{b}},
\end{array}
$$

since $2 \mu-1<0$ for all $\mu<1 / 2$. The last inequality is proved using the following computations. Firstly, the exponent $\frac{1}{\mu_{b}}-\frac{1}{\mu_{a}}$ is negative. Secondly, the right hand side of the inequality is positive for the given $\mu$ values. Now we may fix the value $\mu_{b}$ without loss of generality and with this the left part of the inequation becomes arbitrarily small as $\mu_{a} \rightarrow 0$. Furthermore, we obtain $\frac{\left(1-2 \mu_{b}\right)\left(1-\mu_{b}\right)}{\mu_{b}} \geq 1$ for every $\mu_{b} \leq 1-\frac{1}{\sqrt{2}}$ and it increases asymptotically to infinity as $\mu_{b} \rightarrow 0$. Then there exists some constant $C=C\left(\mu_{b}\right)>1$ such that

$$
\frac{\mu_{a}}{\left(1-2 \mu_{a}\right)\left(1-\mu_{a}\right)} \cdot C<\frac{\mu_{a}}{\left(1-2 \mu_{a}\right)\left(1-\mu_{a}\right)} \frac{\left(1-2 \mu_{b}\right)\left(1-\mu_{b}\right)}{\mu_{b}}
$$

concluding our result. 
We apply the convergence of the absorbing domains to prove that the Baker domain is arbitrarily close to the basins of attraction. The Baker domain $\mathcal{B}$ may or may not be contained in the basins of attraction $\mathcal{A}_{\mu}$. Therefore the kernel convergence does not follows automatically and we prove it using the following proposition.

Proposition 3.16. Let $K$ be an arbitrary compact set contained in $\mathcal{B}_{\lambda}$. Then there exists a $\mu_{*} \in\left(0, \frac{1}{2}\right)$ such that $K \subset \mathcal{A}_{\mu}$ for all $\mu<\mu_{*}$.

Proof. Let $K$ be a compact set contained in $\mathcal{B}_{\lambda}$. Using the definition of an absorbing domain, there exists an $n \in \mathbb{N}$ such that $g_{\lambda}^{n}(K)$ is contained in $\mathcal{M}_{1 / 2}$; see Section 1.7 for the definition. In particular, $g_{\lambda}^{n}(K)$ is also compact. The kernel convergence stated in Theorem 3.3 implies the existence of a $\mu_{1} \in\left(0, \frac{1}{2}\right)$ such that for every $\mu \leq \mu_{1}$ the compact set $g_{\lambda}^{n}(K)$ is contained in $\mathcal{H}_{\eta, \rho}$, the absorbing domain of $\mathcal{A}_{\mu}$. Denote by $U:=U_{\varepsilon}\left(g_{\lambda}^{n}(K)\right)$ an open neighbourhood of $g_{\lambda}^{n}(K)$ such that $U \subset \subset \mathcal{H}_{\eta, \rho}$. The uniform convergence of the functions $g_{\lambda, \mu}$ to $g_{\lambda}$ guarantees the existence of $\mu_{2} \in\left(0, \frac{1}{2}\right)$ such that for every $\mu \leq \mu_{2}, g_{\lambda, \mu}^{n}(K) \subset U$ for every fixed $n \in \mathbb{N}$. Take $\mu \leq \mu_{*}=\min \left\{\mu_{1}, \mu_{2}\right\}$. Hence $g_{\lambda, \mu}^{n}(K)$ and $K$ are contained in $\mathcal{A}_{\mu}$ for every $\mu<\mu_{*}$, which yields the desired result.

Finally, the kernel convergence of $\mathcal{A}_{\mu}$ to $\mathcal{B}_{\lambda}$ follows from the previous results, under the additional condition that there does not exist any further Fatou component.

Theorem 3.17. Let $\lambda \in \mathbb{C}^{*}$ be such that all critical values belong to the Baker domain $\mathcal{B}_{\lambda}$. Then $\mathcal{A}_{\mu}$ converge to $\mathcal{B}_{\lambda}$ as kernel in the sense of Carathéodory.

Proof. The marked point is inherited from the absorbing domains $\mathcal{M}_{1 / 2}$ and $\mathcal{H}_{\eta, \mu}$. The other two statements required for the kernel convergence follow from the previous proposition and properties of the Fatou set. Firstly, the condition on compact sets was proved in Proposition 3.16. Secondly, if there exists a $\mu_{*}$ such that given any open set $U$ is contained in $\mathcal{A}_{\mu}$ for every $\mu<\mu_{*}$, we claim that $U \subset \mathcal{B}_{\lambda}$ as well. If $U \subset \mathcal{A}_{\mu}$, then $U$ is in the Fatou of $g_{\lambda, \mu}$ for every $\mu \leq \mu_{*}$. Then $U$ is contained as well in the Fatou set of $g_{\lambda}$, since $g_{\lambda, \mu}$ converges uniformly to $g_{\lambda}$ and then the iterates of $g_{\lambda}$ also form a normal family on $U$. But all critical points of $g_{\lambda}$ belong to $\mathcal{B}_{\lambda}$, then $\mathcal{F}\left(g_{\lambda}\right)=\mathcal{B}_{\lambda}$ yielding the desired result.

The kernel convergence follows under more general assumptions, provided that we are able to control the critical points of $g_{\lambda}$ and with this, its Fatou components. This will be a consequence of the Hausdorff convergence of the Julia sets as we show below in Section 3.5. The Hausdorff convergence and the kernel convergence are equivalent, if we can prove that all components of $\mathcal{F}\left(g_{\lambda}\right)$ are kernels of the Fatou components of $g_{\lambda, \mu}$ as we discuss in Section 1.9. We can not prove this 
directly. However we firstly show the kernel convergence in the particular case that $g_{\lambda}$ has a parabolic basin. In Section 3.5 we prove the Hausdorff convergence for all parameters $\lambda$ for which $g_{\lambda}$ has no wandering domains and for which we guarantee also kernel convergence for all Fatou components.

\subsubsection{Kernel convergence to the parabolic basin $\mathcal{D}_{p}$}

The second particular case where we show the convergence of Fatou components as kernels in the sense of Carathéodory is when the free critical point $c_{0}$ of the limit functions belongs to the attracting basin of a parabolic fixed point. In this case, the Fatou set $\mathcal{F}\left(g_{\lambda}\right)$ consists of two non-repelling cycles: the Baker domain $\mathcal{B}_{\lambda}$ and the parabolic basin $\mathcal{D}_{p}$.

We proved in Section 3.2 that the function $g_{\lambda}$ has an indifferent fixed point $z_{p}$ which is approximated by a sequence of attracting fixed points $z_{p, \mu}$ of $g_{\lambda, \mu}$ depending on both parameters $\mu$ and $\lambda$. The points $z_{p, \mu}$ are given by the solutions of

$$
\frac{e^{z-z_{p}}}{z_{p}}-\frac{1}{z}=-1+\frac{1}{1-\mu}
$$

for every $z_{p}$ in $\partial D\left(-\frac{2}{3}, \frac{1}{3}\right), \lambda_{p}=\frac{e^{-z_{p}}}{z_{p}}$ and every $\mu \leq \mu_{*}$ for some $\mu_{*} \in\left(0, \frac{1}{2}\right)$.

Assume now that $g_{\lambda}^{\prime}\left(z_{p}\right)$ equals a root of unity, that is, $z_{p}$ is a parabolic fixed point. Then for this parameter $\lambda_{p}$ the free critical point $c_{0}$ belongs to the parabolic basin of $z_{p}$. In this section we prove that the parabolic basin is the kernel of every sequence of the attracting basins of the points $z_{p, \mu}$. With this result we prove below in Section 3.5 that the Hausdorff convergence of Julia set $\mathcal{J}\left(g_{\lambda, \mu}\right)$ to $\mathcal{J}\left(g_{\lambda}\right)$ is satisfied also when the free critical point belongs to a parabolic basin of a rational fixed point.

The proof of Theorem 3.18 consists of several reparametrisations of the functions $g_{\lambda}$ and $g_{\lambda, \mu}$ and constructions of invariant compact sets. Moreover, the proof is based essentially on the Main Lemma 3.20, for which we need to do the reparametrisations. Therefore we firstly present the required tools before we write both proofs, of the theorem and the main lemma, at the end of the section.

During this subsection we omit the parameter $\lambda_{p}$ from the notation, since it is considered arbitrary but fixed and depending on the parabolic fixed point $z_{p}$.

We start with the reparametrisation of the functions $g_{\lambda}$ and $g_{\lambda, \mu}$ by sending the fixed points $z_{p}$ and $z_{p, \mu}$ to the origin. Then we express the reparametrisated functions $\widehat{g}$ and $\widehat{g_{\mu}}$ by their Taylor expansion in a small neighbourhood $U$ of zero. 
The fixed point $z_{p}$ has multiplier $M_{p}$, which is a $q$-th root of unity. Hence we may substitute the function $\widehat{g}$ by its $q$-th iterate and the multiplier $M_{p}:=M_{0}=1$. It follows that $M_{p, \mu}:=M_{\mu} \in \mathbb{D}$ and convergences to 1 . We prove in Proposition 3.19 that this convergence is not tangent to $\partial \mathcal{D}$.

Afterwards we lift the neighbourhood $U$ of the origin to a neighbourhood of $\infty$ by a biholomorphic mapping to the Riemann surface of the $m$-th root, where $m$ denotes the number of attracting petals of the parabolic fixed point. We show that any compact set $K \subset U$ which is invariant under $\widehat{g}$ is also invariant under $\widehat{g_{\mu}}$. In other words, $K$ is also contained in the basin of attraction $\mathcal{A}_{p, \mu}$. To this aim, we construct an appropriate neighbourhood $\mathcal{W}_{\mu}$ of infinity using the lifting and which contains $K$ such that it remains invariant under $\widehat{g}$ and $\widehat{g_{\mu}}$. Finally we prove the theorem using all these results

Let $\mathcal{D}_{p}^{*}$ denote the immediate attracting basin of the parabolic fixed point $z_{p}$. The set $\mathcal{D}_{p}^{*}$ is defined as the union of the largest open sets $U_{j}$ called petals, for $j \in\{1, \ldots, m\}$, satisfying $\bigcap_{j=1}^{m}\left(\overline{U_{j}}\right)=z_{p}$ and $g_{\lambda}^{n}(z) \rightarrow z_{p}$ for every $z \in U_{j}$. Then denote by $\mathcal{D}_{p}$ the parabolic basin of $z_{p}$, that is, all points $z \in \mathbb{C}^{*}$ that are eventually mapped into $\mathcal{D}_{p}^{*}$. In the same way, denote by $\mathcal{A}_{p, \mu}$ (resp. $\mathcal{A}_{p, \mu}^{*}$ ) the (immediate) basin of attraction of the point $z_{p, \mu}$. The main result is the following.

Theorem 3.18. Let $g_{\lambda}$ and $g_{\lambda, \mu}$ be as described above. Then for every parabolic fixed point $z_{p} \in \partial D\left(-\frac{2}{3}, \frac{1}{3}\right)$ and every $\mu \leq \mu_{*}$, the attracting basins $\mathcal{A}_{p, \mu}$ converge as kernels to the parabolic basin $\mathcal{D}_{p}$ as $\mu$ tends to zero.

Suppose the parameter $\mu_{*}$ is such that the convergence of $z_{p, \mu}$ to $z_{p}$ is guaranteed for every $\mu \leq \mu_{*}$. We proceed with the analysis of the function

$$
g(z)=z-1+e^{z-z_{p}} \frac{z}{z_{p}}
$$

and its approximation by the functions

$$
g_{\mu}(z)=(1-\mu)\left(z-1+e^{z-z_{p}} \frac{z}{z_{p}}\right)
$$

considering the limit as $\mu$ tends to zero.

We proved in Section 3.2 that the function $g_{\mu}$ has an attracting fixed point $z_{p, \mu}$ converging to $z_{p}$ as $\mu \rightarrow 0$ in the general case that $\left|g^{\prime}\left(z_{p}\right)\right|=1$. In particular, it applies to the case that $g^{\prime}\left(z_{p}\right)$ is a root of unity, that is, its argument is a rational number.

Write $M_{0}:=g^{\prime}\left(z_{p}\right)$ and $M_{\mu}:=g_{\mu}^{\prime}\left(z_{p, \mu}\right)$ for the multipliers at the fixed points. 
Then $M_{0}=2+\frac{1}{z_{p}}$ and

$$
M_{\mu}=(1-\mu)\left(2+\frac{1}{z_{p, \mu}}\right)+\mu\left(z_{p, \mu}+1\right) \approx\left(2+\frac{1}{z_{p}}\right)\left(1+\mu Q\left(z_{p}\right)\right)
$$

with $Q\left(z_{p}\right)=\left(z_{p}-1-\frac{1}{z_{p}}-\frac{1}{z_{p}+1}\right)\left(2+\frac{1}{z_{p}}\right)^{-1}$ computed by using the approximation for the fixed point $z_{p, \mu} \approx z_{p}+\mu\left(\frac{z_{p}^{2}}{z_{p}+1}\right)$ carried out in Theorem 3.10 .

We now show that the convergence of $M_{\mu} \in \mathbb{D}$ to $M_{0}$ is not tangential to $\partial \mathbb{D}$ for $M_{0} \neq 1$. That is, $\left|\arg \left(M_{0}-M_{\mu}\right)-\arg \left(M_{0}\right)\right| \leq \frac{\pi}{2}-C$ for some $C=C\left(z_{p}\right)>0$.

Proposition 3.19. Let $M_{0}$ and $M_{\mu}$ denote the multipliers of the parabolic and attracting fixed points respectively, with $M_{0} \neq 1$. Then $M_{\mu}$ lies in $\mathbb{D}$ and the convergence of $M_{\mu}$ to $M_{0}$ is not tangential to $\partial \mathbb{D}$.

Proof. The fact that $M_{\mu} \in \mathbb{D}$ follows immediately from Theorem 3.10 . To prove that the convergence is not tangential, we show that $\left|\arg \left(M_{0}-M_{\mu}\right)-\arg M_{0}\right|<\frac{\pi}{2}$. For $\mu \in \mathbb{R}$ we obtain

$$
\begin{aligned}
\left|\arg \left(M_{0}-M_{\mu}\right)-\arg M_{0}\right| & =\left|\arg \left(\frac{M_{0}-M_{\mu}}{M_{0}}\right)\right| \approx\left|\arg \left(1-\left(1+\mu Q\left(z_{p}\right)\right)\right)\right| \\
& =\left|\arg \left(-\mu Q\left(z_{p}\right)\right)\right|=\left|\arg \left(Q\left(z_{p}\right)\right)\right| .
\end{aligned}
$$

Finally, $\left|\arg \left(Q\left(z_{p}\right)\right)\right| \leq \frac{\pi}{2}-C$ with $C=C\left(x_{p}\right)>0$ for any given value $x_{p}>-1$.

We remark that $C\left(z_{p}\right)$ tends to zero as the point $z_{p}$ tends to -1 on $\partial D\left(-\frac{2}{3}, \frac{1}{3}\right)$. However, for our purposes we may take $C\left(z_{p}\right)$ arbitrarily small. In other words and taking in consideration Lemma 3.11 and Proposition 3.13, we conclude that even though $\left|\operatorname{Im} Q\left(z_{p}\right)\right|$ is not bounded, we find for every fixed point $z_{p} \in D\left(-\frac{2}{3}, \frac{1}{3}\right)$ a sufficiently large constant $L>0$ and $\delta>0$ such that if $\left|z_{p}-1\right| \geq \delta$, then $\left|\operatorname{Im} Q\left(z_{p}\right)\right|<L$.

In order to simplify the proof of Theorem 3.18 , we reparametrise the function $g$ by sending the fixed point $z_{p}$ to the origin. Let $\psi(z):=z-z_{p}, z_{p} \neq-1$, and define the function $\widehat{g}$ as follows

$$
\begin{aligned}
\widehat{g}(z) & :=\psi \circ g \circ \psi^{-1}(z) \\
& =z-1+e^{z}\left(\frac{z+z_{p}}{z_{p}}\right) .
\end{aligned}
$$


The function $\widehat{g}$ has $z_{0}=0$ as a fixed point with multiplier $M_{0}$. In other words, the translation leaves the multiplier of $g$ invariant at the fixed point. This is clear, since $\widehat{g}^{\prime}(z)=1+e^{z}\left(\frac{z+z_{p}+1}{z_{p}}\right)$ which equals $2+\frac{1}{z_{p}}$ at $z_{0}=0$.

Furthermore, we can use the Taylor expansion of $\widehat{g}$ at zero to simplify the notation and computations in the proof obtaining

$$
\begin{aligned}
\widehat{g}(z) & =\left(2+\frac{1}{z_{p}}\right) z+\left(1+\frac{2}{z_{p}}\right) \frac{z^{2}}{2}+\cdots+\left(1+\frac{n}{z_{p}}\right) \frac{z^{n}}{n !}+\cdots \\
& =M_{0} z+\sum_{n=2}^{\infty} a_{n} z^{n}
\end{aligned}
$$

with $a_{n}=\frac{1}{n !}\left(1+\frac{n}{z_{p}}\right)$ and $z$ close to the origin.

In an analogue way we reparametrise the function $g_{\mu}(z)$ for every $\mu \in\left(0, \mu_{*}\right]$ by conjugating with the function $\psi_{\mu}(z)=z-z_{p, \mu}$ as

$$
\begin{aligned}
\widehat{g_{\mu}}(z) & :=\psi_{\mu} \circ g_{\mu} \circ \psi_{\mu}^{-1}(z) \\
& =(1-\mu)\left(z+z_{p, \mu}-1+e^{z+z_{p, \mu}-z_{p}}\left(\frac{z+z_{p, \mu}}{z_{p}}\right)\right)-z_{p, \mu} .
\end{aligned}
$$

In this case we also obtain that $\widehat{g_{\mu}}$ fixes the origin and the multiplier remains invariant at $z_{0}=0$. Computing the derivative we obtain

$$
\begin{aligned}
& {\widehat{g_{\mu}}}^{\prime}(z)=(1-\mu)\left(1+e^{z+z_{p, \mu}-z_{p}}\left(\frac{z+z_{p, \mu}+1}{z_{p}}\right)\right) \text { and } \\
& {\widehat{g_{\mu}}}^{\prime}(0)=(1-\mu)\left(1+e^{z_{p, \mu}-z_{p}}\left(\frac{z_{p, \mu}+1}{z_{p}}\right)\right)
\end{aligned}
$$

which coincides with $g_{\mu}^{\prime}\left(z_{p, \mu}\right)=M_{\mu}$ comparing with Equation 3.5). Since $z_{p, \mu}$ is a fixed point of $g_{\mu}$, it satisfies the equality $\frac{e^{z_{p, \mu}-z_{p}}}{z_{p}}=\frac{\mu}{1-\mu}+\frac{1}{z_{p, \mu}}$. Hence we may rewrite

$$
{\widehat{g_{\mu}}}^{\prime}(z)=(1-\mu)\left(1+e^{z}\left(\frac{\mu}{1-\mu}+\frac{1}{z_{p, \mu}}\right)\left(z+z_{p, \mu}+1\right)\right) .
$$

Finally, the Taylor expansion of $\widehat{g_{\mu}}(z)$ at 0 is given by $\widehat{g_{\mu}}(z)=M_{\mu} z+\sum_{n=2}^{\infty} a_{n, \mu} z^{n}$ with

$$
\begin{aligned}
a_{n, \mu} & =(1-\mu)\left(\frac{\mu}{1-\mu}+\frac{1}{z_{p, \mu}}\right)\left(\frac{z_{p, \mu}+n}{n !}\right) \\
& =\frac{1-\mu}{n !}\left(z_{p, \mu}\left(-1+\frac{1}{1-\mu}\right)+n\left(-1+\frac{1}{1-\mu}\right)+1+\frac{n}{z_{p, \mu}}\right),
\end{aligned}
$$


using the expression (3.18) for the derivative. We claim that the coefficients of the Taylor expansion converge, that is, for every $n \geq 2$ we have that $a_{n, \mu}$ tends to $a_{n}$ as $\mu \rightarrow 0$. As we take the limit $\mu \rightarrow 0$, then $a_{n, \mu}$ tends to $\frac{1}{n !}\left(1+\frac{1}{z_{p, \mu}}\right)$ and $z_{p, \mu}$ tends to $z_{p}$ yielding the convergence.

We continue by proving that any compact set $K \subset \mathcal{D}_{p}$ is contained in $\mathcal{A}_{p, \mu}$ and is invariant under $g_{\lambda, \mu}$ as well. We firstly state in the Main Lemma below that if $K$ is close enough to $z_{p}$, then $K$ is also contained in $\mathcal{A}_{p, \mu}^{*}$. Later we show in the proof of the theorem that this implies the result for an arbitrary compact set $K$ in the parabolic basin. The proof of the Main Lemma is also written at the end of the section, together with the proof of Theorem 3.18 .

Lemma 3.20 (Main Lemma for Parabolic Basins). Let $z_{p}$ be a parabolic fixed point of $g$ and $U$ a small neighbourhood of $z_{p}$. Then for every compact set $K \subset U \cap \mathcal{D}_{p}^{*}$ there exists an $\mu_{*} \in\left(0, \frac{1}{2}\right)$ such that $K \subset \mathcal{A}_{p, \mu}^{*}$ for all $\mu<\mu_{*}$.

The invariance under the functions $g_{\lambda}$ and $g_{\lambda, \mu}$ is equivalent to the invariance under the reparametrised functions $\widehat{g}$ and $\widehat{g_{\mu}}$. To prove the invariance under $\widehat{g_{\mu}}$ we use the fact that $\widehat{g}$ behaves like a translation when we send the neighbourhood $U$ of the origin to a neighbourhood $\mathcal{W}_{\lambda}$ of infinity. Then we show that the functions $\widehat{g_{\mu}}$ are small perturbations of this translation, leaving $\mathcal{W}_{\lambda}$ also invariant and with it, the compact set $K$.

Until now, we worked with an arbitrary parabolic fixed point $z_{p} \neq 1$ such that $z_{p}$ lies on the boundary of $D\left(-\frac{2}{3}, \frac{1}{3}\right)$ and later with its translation to the origin. In particular, its multiplier lies on $\partial \mathbb{D}$ and it is a $q$-th root of unity, for some $q \in \mathbb{N}$. Consider now $z_{p}$ fixed but arbitrary under the restrictions above. We substitute the reparametrised function $\widehat{g}$ by its $q$-th iterate. This implies that the multiplier $\widehat{M}_{0}:=1$ and the function is of the form:

$$
\widehat{g}(z)=z+\widehat{a}_{m+1} z^{m+1}+\widehat{a}_{m+2} z^{m+2}+\cdots
$$

with $\widehat{a}_{m+1} \neq 0$ and where $m \geq 1$ is called the multiplicity of $z_{p}$. The number $m$ describes in particular that the parabolic basin of $z_{p}$ consists of $m$ petals; see Theorem 10.7 in [51].

Analogously, we substitute $\widehat{g_{\mu}}(z)$ by its $q$-th iterate. The multiplier $\widehat{M}_{\mu}$ is still contained in $\mathbb{D}$ and $\widehat{M}_{\mu} \rightarrow 1$ as $\mu \rightarrow 0$. Since we translate and reparametrise by conformal mappings, the convergence of $\widehat{M}_{\mu}$ to $\widehat{M}_{0}=1$ is also not tangent to $\partial \mathbb{D}$. Furthermore, the convergence of the coefficients $\widehat{a}_{n, \mu}$ to $\widehat{a}_{n}$ is also preserved. The approximating functions are of the form

$$
\widehat{g_{\mu}}(z)=\widehat{M}_{\mu} z+\widehat{a}_{m+1, \mu} z^{m+1}+\ldots
$$


with $\widehat{a}_{m+1, \mu} \neq 0, \widehat{M}_{\mu} \in \mathbb{D}$ and $\widehat{M}_{\mu}$ tending to 1 as $\mu$ tends to 0 . Notice that the coefficients $\widehat{a}_{k, \mu}$ equal zero for $1 \leq k \leq m$. The index $m$ is the multiplicity of the fixed point $z_{p}=0$, meaning the order of annulation of $\widehat{g}\left(z_{p}\right)=z_{p}$. Since the functions $\widehat{g_{\mu}}$ converge uniformly to $\widehat{g}$, the order of the fixed point $z_{p, \mu}=0$ must be preserved by $\widehat{g_{\mu}}$ for every $\mu \leq \mu_{0}$ and $\mu_{0}$ sufficiently small. In both cases the higher terms are bounded and depend on $z_{p}$ for $g$ and on $z_{p, \mu}$ and $\mu$ for $g_{\mu}$. For $\mu=0$ we set $\widehat{g}_{0}(z)=\widehat{g}(z)$ and for simplicity we maintain the notation $a_{k, \mu}$ instead of $\widehat{a}_{k, \mu}, M_{\mu}$ instead of $\widehat{M}_{\mu}$ keeping in mind that $M_{0}=1$.

Consider now an arbitrary but fixed neighbourhood $U$ of the origin and proceed with the construction of a neighbourhood of $\infty$. To this end we make a change of coordinates applying a method utilised by Douady and Hubbard in [24] and by Kriete in [46] among others.

The function $\varphi: z \mapsto z^{-m}$ is a holomorphic mapping from $\mathbb{C}^{*}$ to itself, but it is not injective in any neighbourhood of the origin. Therefore we consider the Riemann surface of the $m$-th root denoted by $S_{m}^{*}$. In the case $m>1$ the immediate parabolic basin consists of more than one simply connected domain, namely of $m$ petals.

The mapping $\varphi$ defines a biholomorphic mapping $\phi: \mathbb{C}^{*} \rightarrow S_{m}^{*}$ and the projection $\rho: S_{m}^{*} \rightarrow \mathbb{C}^{*}$. Hence it holds that $\rho \circ \phi=\varphi$ and diagram below commutes.

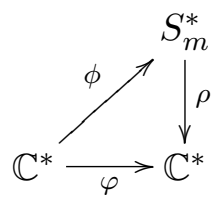

In other words, $\phi$ is a lifting of the mapping $\varphi$ to the Riemann surface $S_{m}^{*}$. Moreover, $\phi$ maps a punctured neighbourhood of the origin to a punctured neighbourhood of infinity biholomorphically. We define for every $\mu \in\left[0, \mu_{*}\right]$ the function

$$
f_{\mu}:=\left.\phi \circ \widehat{g_{\mu}}\right|_{U} \circ \phi^{-1} \text {. }
$$

The function $f_{\mu}$ is a biholomorphic function, that is, it is well defined on a punctured neighbourhood of $\infty$ on the Riemann surface $S_{m}^{*}$. For any $r>0$ sufficiently large write $\Omega_{r}:=\left\{\xi \in S_{m}^{*}:|\xi|>r\right\}$. Then $\Omega_{r}$ describes a disc around $\infty$ in $S_{m}^{*}$. Let now $r$ and $R$ be sufficiently large with $0<R<r<\infty$. It follows that the function $f_{0}$ maps $\Omega_{r}$ into $\Omega_{R}$ holomorphically, since $\varphi$ acts like an expansion close to the origin. 
Lemma 3.21. The expression of the function $f_{\mu}$ in the $\xi$-coordinates on $S_{m}^{*}$ close to $\infty$ is given by

$$
\xi \mapsto M_{\mu}^{-m} \xi-1+\mathcal{O}\left(\xi^{-\frac{1}{m}}\right) \quad \text { for every } \quad \mu \in\left[0, \mu_{*}\right] .
$$

Recall that $\varphi(z)=z^{-m}$ and $\varphi^{-1}(z)=z^{-\frac{1}{m}}$. Then after some computations, it follows for any point $z$ in a small neighbourhood of zero

$$
\begin{aligned}
\varphi \circ \widehat{g_{\mu}} \circ \varphi^{-1}(z) & =\left(M_{\mu} z^{-\frac{1}{m}}+a_{m+1, \mu}\left(z^{-\frac{1}{m}}\right)^{m+1}+\mathcal{O}\left(\left(z^{-\frac{1}{m}}\right)^{m+2}\right)\right)^{-m} \\
& =\left(M_{\mu} z^{-\frac{1}{m}}\right)^{-m}\left(1+\frac{a_{m+1, \mu}}{M_{\mu}}\left(z^{-\frac{1}{m}}\right)^{m}+\mathcal{O}\left(\left(z^{-\frac{1}{m}}\right)^{m+1}\right)\right)^{-m} .
\end{aligned}
$$

Applying Taylor expansion at zero to the term on the right side we obtain

$$
\begin{aligned}
\varphi \circ \widehat{g_{\mu}} \circ \varphi^{-1}(z) & =\left(M_{\mu}^{-m} z\right)\left(1-\frac{m a_{m+1, \mu}}{M_{\mu}} z^{-1}+\mathcal{O}\left(z^{\frac{-m-1}{m}}\right)\right) \\
& =M_{\mu}^{-m} z-m M_{\mu}^{-m-1} a_{m+1, \mu}+\mathcal{O}\left(z^{-\frac{1}{m}}\right) .
\end{aligned}
$$

Furthermore, from the definition of $f_{\mu}=\phi \circ \widehat{g_{\mu}} \circ \phi^{-1}$ together with $\phi=\rho^{-1} \circ \varphi$ we can rewrite the function $f_{\mu}$ in the new coordinates. Given a large $r$ for each $\xi \in \Omega_{r} \subset S_{m}^{*}$ there exists a $z$ close enough to zero with $\rho(\xi)=z$. It holds that $\widehat{g_{\mu}}(z)$ is also close enough to zero. Hence $\xi=\rho^{-1}(z)$ and $f_{\mu}(\xi)=\rho^{-1}\left(\widehat{g_{\mu}}(z)\right)$ lie both on the same leaf of $S_{m}^{*}$. Finally we obtain the expected expression by an appropriate reparametrisation such that the constant $-m M_{\mu}^{-m-1} a_{m+1, \mu}$ is replaced by -1 .

The following constructions and lemmas are presented and proved by Kriete in his doctoral thesis [46]. Kriete proved the results for an analytic family of rational functions depending on a parameter $\lambda \in \mathbb{D}$, even though we reach the same expression (3.19) as he did. It means that both families have the same analytic expression in a small neighbourhood of the parabolic fixed point and his results apply also for our functions. We rewrite the results with our notation and omit most of the proofs. Nevertheless we write some sketches of them necessary for the understanding of the Main Lemma's proof and adapted to transcendental functions if it is necessary.

We start by constructing an appropriate neighbourhood of $\infty$ via $\Omega_{r}(\infty)$ and its projection $\rho\left(\Omega_{r}(\infty)\right) \subset \mathbb{C}^{*}$ which will be invariant under the perturbation $\widehat{g_{\mu}}$ of $\widehat{g}$. Let $K$ be an arbitrary compact set in $\mathcal{D}_{p}^{*}$. By definition, for every point $z_{0}$ in $\mathcal{D}_{p}^{*}$ there exists an $n_{0} \in \mathbb{N}$ such that $\widehat{g}_{0}^{n}\left(z_{0}\right) \in U \cap \mathcal{D}_{p}^{*}$ for every $n \geq n_{0}$. Moreover, there exists a neighbourhood $V$ of $z_{0}$ such that $\widehat{g}_{0}^{n}(V) \subset \subset U \cap \mathcal{D}_{p}^{*}$ for every $n \geq n_{0}$. Then 
for the compact set $K \subset \mathcal{D}_{p}^{*}$ we can choose an $n_{1} \in \mathbb{N}$ such that $\widehat{g}_{0}^{n}(K) \subset \subset U \cap \mathcal{D}_{p}^{*}$ for every $n \geq n_{1}$. Hence, we continue with the proof on an arbitrary compact set $K \subset U \cap \mathcal{D}_{p}^{*}$, that is, close to the origin.

The previous arguments in particular imply that $f_{0}^{n}(\phi(K)) \subset \Omega_{r}$ for every $n \in \mathbb{N}$ and an appropriate $r>0$. Recall that $f_{0}(\xi) \approx \xi-1+\mathcal{O}\left(\xi^{-\frac{1}{m}}\right)$. For sufficiently large $r>0$ and $\xi \in \Omega_{r}$ it holds that $\operatorname{Re}\left(\rho\left(f_{0}(\xi)\right)\right)<\operatorname{Re}(\rho(\xi))-\frac{1}{2}$. Then for every $n \in \mathbb{N}$ and any $\xi \in \phi(K)$ this implies that

$$
\operatorname{Re}\left(\rho\left(f_{0}^{n}(\xi)\right)\right)<\operatorname{Re}(\rho(\xi))-\frac{n}{2} .
$$

With this, we may and will assume that $\operatorname{Re}(\rho(\xi))<-r$ for an arbitrary $\xi \in$ $\left.f_{0}^{n}(\phi(K))\right)$ and $n \in \mathbb{N}$.

Consider the multiplier $M_{\mu}$ at the fixed point $z_{p, \mu}=0$ for $\mu \in\left[0, \mu_{*}\right]$. Recall that $M_{0}=1$ and that $M_{\mu} \in \mathbb{D}$ converges to $1 \in \partial \mathbb{D}$ non-tangentially. Hence we assume that $M_{\mu}$ lies on a curve contained in a triangle $\Delta$ symmetric with respect to the real axis and such that $\partial \Delta \cap \partial \mathbb{D}=\{1\}$. Let $\beta$ denote the angle between the real axis and one of the sides of $\Delta$. Since $\Delta \subset \mathbb{D}$ it holds that $\beta \in\left(0, \frac{\pi}{2}\right)$. Define $\eta:=\min \left\{\frac{\beta}{2}, \frac{1}{2}\left(\frac{\pi}{2}-\beta\right)\right\}$ and choose a sufficiently large $r$ such that $\xi \in \Omega_{r}$ satisfies $\left|\operatorname{Im} \rho\left(f_{0}(\xi)\right)-\operatorname{Im} \rho(\xi)\right|<\frac{1}{2} \tan \frac{\eta}{2}$. Then for any $\xi \in \phi(K)$ and $n \in \mathbb{N}$ we obtain

$$
\pi-\frac{\eta}{2}<\arg \left(\rho\left(f_{0}^{n}(\xi)\right)-\rho(\xi)\right)<\pi+\frac{\eta}{2} .
$$

Equations 3.20 and 3.21 ensure the existence of $n_{0} \in \mathbb{N}$ such that

$$
\pi-\eta<\arg \left(\rho\left(f_{0}^{m}(\xi)\right)\right)<\pi+\eta
$$

for all $m \geq n_{0}$ and $\xi \in \phi(K)$. Therefore we may restrict the proof of the invariance for points $\xi$ in a compact set $K \subset U \cap \mathcal{D}_{p}^{*}$ such that

$$
\phi(K) \subset \mathcal{B}^{\prime}(r, \beta):=\left\{\xi \in \Omega_{r}:|\pi-\arg (\rho(\xi))|<\eta \text { and } \operatorname{Re}(\rho(\xi))<-r\right\} .
$$

We denote by $\mathcal{B}(r, \beta):=\rho\left(\mathcal{B}^{\prime}(r, \beta)\right)$. The results above yield the proof of the following lemma.

Lemma 3.22. The domain $\mathcal{B}^{\prime}(r, \beta)$ is invariant under $f_{0}$.

The domain $\mathcal{B}^{\prime}(r, \beta)$ is invariant under $f_{0}$ by construction and contains the compact set $\phi(K)$. The action of $f_{0}$ on $\xi$ is mainly a translation by -1 , since $M_{0}=1$. In contrast to this, the function $f_{\mu}$ additionally acts as a small rotation by $M_{\mu}^{-m}$. To find an invariant domain under $f_{\mu}$ we extend $\mathcal{B}(r, \beta)$ by adding the domain $\mathcal{V}_{\mu}$ defined as the complement of a bounded closed disc which intersects $\mathcal{B}(r, \beta)$. 
We claim that the domain $\mathcal{W}_{\mu}^{\prime}:=\left\{\xi: \rho(\xi) \in \mathcal{B}(r, \beta) \cup \mathcal{V}_{\mu}\right\}$ is invariant under $f_{\mu}$ for every $\mu \in\left(0, \mu_{*}\right]$. To prove this we firstly show the invariance under the function $h_{\mu}$ defined as $h_{\mu}(\xi):=M_{\mu}^{-1} \xi-1$ for points $\xi \in \mathcal{W}_{\mu}^{\prime}$. In the $\xi$-coordinates, the function $f_{\mu}$ is a small perturbation of $h_{\mu}$ just by adding $\mathcal{O}\left(\xi^{-\frac{1}{m}}\right)$. Afterwards we prove that the invariance of $\mathcal{W}_{\mu}$ is preserved by small perturbations of $h_{\mu}$ finally implying that $\rho^{-1}\left(\mathcal{W}_{\mu}\right)$ is invariant under $f_{\mu}$.

We construct the domain $\mathcal{V}_{\mu}$ by defining its intersection with $\mathcal{B}(r, \beta)$. Denote each component of $\partial \mathcal{B}(r, \beta)$ by

$$
\begin{aligned}
& \mathcal{B}_{0}:=\partial \mathcal{B}(r, \beta) \cap\{\operatorname{Re} \xi=-r\} \\
& \mathcal{B}_{1}:=\partial \mathcal{B}(r, \beta) \cap\{\arg \xi=\pi+\eta\} \\
& \mathcal{B}_{2}:=\partial \mathcal{B}(r, \beta) \cap\{\arg \xi=\pi-\eta\} .
\end{aligned}
$$

Lemma 3.23. Let $h_{\mu}(\xi)=M_{\mu}^{-1} \xi-1$. Then $h_{\mu}(\xi) \in \mathcal{B}(r, \beta)$ holds for every $\xi$ in $\partial \mathcal{B}(r, \beta)$. Furthermore there exist $\mu_{3}>0, \delta=\delta\left(\mu_{3}\right)$ and some constant $C>0$ such that if $\left|M_{\mu}-M_{0}\right|<\delta$ for every $\mu \leq \mu_{3}$ then $d\left(h_{\mu}\left(\mathcal{B}_{0} \cup \mathcal{B}_{1} \cup \mathcal{B}_{2, \mu}\right), \partial \mathcal{B}(r, \beta)\right)>C_{\mathcal{B}}$.

The proof of Lemma 3.23 is written in detail by Kriete in [46]. Here we provide a sketch of the proof since some of the arguments are helpful for the construction of the domain $\mathcal{V}_{\mu}$.

Fix $r>0$ sufficiently large. It is clear that $h_{0}\left(\mathcal{B}_{0}\right) \subset \mathcal{B}(r, \beta)$ since $M_{0}=1$ and that $d\left(h_{0}\left(\mathcal{B}_{0}\right), \partial \mathcal{B}(r, \beta)\right)$ does not depend on $r$. Then it follows that $h_{\mu}\left(\mathcal{B}_{0}\right) \subset \mathcal{B}(r, \beta)$ and $d\left(h_{\mu}\left(\mathcal{B}_{0}\right), \partial \mathcal{B}(r, \beta)\right)>C_{0}$ for every $\left|M_{\mu}-M_{0}\right|<\delta_{0}$ with $\mu \leq \mu_{0}, \delta_{0}=\delta_{0}\left(\mu_{0}\right)$ and some appropriate constant $C_{0}$ independent of $r$.

Now consider $\delta_{1} \in\left(0, \min \left\{\tan \eta, \delta_{0}\right\}\right)$ with $0 \leq \arg M_{\mu}<\eta$. Then it holds that $0 \geq \arg M_{\mu}^{-1}>-\eta$. For points $\xi \in \mathcal{B}_{1}$ we obtain $\pi<\arg \left(M_{\mu}^{-1} \xi\right) \leq \pi+\eta$ and $\operatorname{Re}\left(M_{\mu}^{-1} \xi\right)<\operatorname{Re}(\xi)$. This implies $\operatorname{Im}\left(h_{\mu}(\xi)\right) \geq \operatorname{Im} \xi$ and $\operatorname{Re}\left(h_{\mu}(\xi)\right) \leq \operatorname{Re}(\xi)-1$ finally implying that $h_{\mu}(\xi) \in \mathcal{B}(r, \beta)$. We also find a constant $C_{1}>0$ independent from $r$ such that $d\left(h_{\mu}\left(\mathcal{B}_{1}\right), \partial \mathcal{B}(r, \beta)\right)>C_{1}$.

We continue by showing the invariance of the upper boundary component. For $\xi \in \mathcal{B}_{2}$ it suffices that $\pi-\eta<\arg \left(h_{\mu}(\xi)-\xi\right)<\pi$ holds in order to prove $h_{\mu}(\xi) \in \mathcal{B}(r, \beta)$. The claim follows from the inequations $\operatorname{Re}\left(h_{\mu}(\xi)-\xi\right) \leq-1$ and $\operatorname{Im}\left(h_{\mu}(\xi)-\xi\right) \leq \tan \frac{\eta}{2}$, which we prove now.

Write $M_{\mu}=1-a+i b$ with $a \in(0,1)$ and $b \in \mathbb{R}$ satisfying $|b| \leq a \tan \beta$. It is possible to show that for $\xi \in \mathcal{B}_{2}$ and a small $a \in(0,1)$ we obtain $\operatorname{Re}\left(M_{\mu}^{-1} \xi\right) \leq \operatorname{Re}(\xi)$. Furthermore there exists $\delta_{2} \in\left(0, \delta_{1}\right)$ such that for $\left|M_{\mu}-M_{0}\right|<\delta_{2}$ we obtain $\tan \eta<(1-a) \cot \beta-b$. Then for these values of $M_{\mu}$ the inequation

$$
\operatorname{Re}\left(h_{\mu}(\xi)-\xi\right) \leq-1
$$


holds. The inequation

$$
\operatorname{Im}\left(h_{\mu}(\xi)-\xi\right) \leq \tan \frac{\eta}{2}
$$

is only satisfied for a subset $\mathcal{B}_{2, \mu}$ of $\mathcal{B}_{2}$ defined as follows. For $b \geq 0$ we obtain $\operatorname{Im}\left(M_{\mu}^{-1} \xi\right) \geq \operatorname{Im} \xi$ and $\arg \left(h_{\mu}(\xi)-\xi\right)<\pi$. Using Equation 3.22 we compute

$$
0<\tan \left(\pi-\arg \left(h_{\mu}(\xi)-\xi\right)\right)=-\frac{\operatorname{Im}\left(h_{\mu}(\xi)-\xi\right)}{\operatorname{Re}\left(h_{\mu}(\xi)-\xi\right)} \leq \operatorname{Im}\left(h_{\mu}(\xi)-\xi\right) .
$$

If $\operatorname{Im}\left(h_{\mu}(\xi)-\xi\right) \leq \tan \frac{\eta}{2}$ is satisfied, then it yields $\pi-\eta<\arg \left(h_{\mu}(\xi)-\xi\right)<\pi$ using the monotony of the tangent function. Hence we conclude that $h_{\mu}(\xi) \in \mathcal{B}(r, \beta)$.

By further computations we obtain by writing $\xi=-x+i y$ with $x, y>0$ that

$$
\begin{aligned}
\operatorname{Im}\left(h_{\mu}(\xi)-\xi\right) & =\frac{x \cdot b+y(1-a)-y\left((1-a)^{2}+b^{2}\right)}{\left|M_{\mu}\right|^{2}} \\
& =\frac{x\left(b+a(1-a) \tan \eta-b^{2} \tan \eta\right)}{\left|M_{\mu}\right|^{2}}
\end{aligned}
$$

for $y=x \tan \eta$. Furthermore, Inequation $(3.23)$ holds for $\left|M_{\mu}-M_{0}\right|=\left|M_{\mu}-1\right|<$ $\delta_{2}$ and $b \leq a \tan \beta$ provided the points $\xi \in \mathcal{B}_{2}$ satisfy

$$
x \cdot a\left(\frac{\tan \beta+\tan \eta+a \tan ^{2} \beta \cdot \tan \eta}{\left|1-\delta_{2}\right|^{2}}\right) \leq \tan \frac{\eta}{2} .
$$

Approximating the second factor on the left hand side of the inequation by an appropriate constant $L$ as an upper bound we define $\zeta_{2}=\zeta_{2}(\mu) \in \mathcal{B}_{2}$ such that $x \cdot a \cdot L=\tan \frac{\eta}{2}$ and

$$
\mathcal{B}_{2, \mu}:=\left\{\xi \in \mathcal{B}_{2}: \operatorname{Re}(\xi) \geq \operatorname{Re}\left(\zeta_{2}\right)\right\}
$$

For a point $\xi \in \mathcal{B}_{2, \mu}$ it is possible to approximate the distance of its value $h_{\mu}(\xi)$ to the boundary as $d\left(h_{\mu}(\xi), \mathcal{B}_{0}\right)>1, d\left(h_{\mu}(\xi), \mathcal{B}_{1}\right)>r \cdot \tan \eta$ and $d\left(h_{\mu}(\xi), \mathcal{B}_{2}\right)>\tan \frac{\eta}{2}$. We may assume w.l.o.g. that $r \geq 1$ and with this we set $C_{2}:=\min \left\{1, \tan \frac{\eta}{2}\right\}$ for which we have $d\left(h_{\mu}(\xi), \partial \mathcal{B}(r, \beta)\right)>C_{2}$. To complete the hypothesis of Lemma 3.23 we set the constant $C_{\mathcal{B}}:=\min \left\{C_{0}, C_{1}, C_{2}\right\}$.

We define the domain $\mathcal{V}_{\mu}$ using the point $\zeta_{2}$. Firstly consider the repelling fixed point of $h_{\mu}$ given by $\xi_{0}=\frac{M_{\mu}}{1-M_{\mu}}$. We choose the circle $\gamma_{\mu}$ with centre at $\xi_{0}$ and radius $R_{\mu}=\left|\zeta_{2}-\xi_{0}\right|$ as the boundary of $\mathcal{V}_{\mu}$. That is to say, we define $\mathcal{V}_{\mu}$ as the unbounded component of $\mathbb{C}^{*} \backslash \gamma_{\mu}$. It is clear that $\partial \mathcal{V}_{\mu} \cap \partial \mathcal{B}_{2}=\zeta_{2}$. 
Lemma 3.24. The domain $\mathcal{V}_{\mu}$ is invariant under the function $h_{\mu}$. Furthermore, there exist $\delta_{3}=\delta_{3}\left(\mu_{3}\right)>0$ and a positive constant $C_{\mathcal{V}}$ independent of $r$ such that if $\left|M_{\mu}-M_{0}\right|<\delta_{3}$ for every $\mu \leq \mu_{3}, \mu_{3} \in\left(0, \mu_{2}\right)$, then $d\left(h_{\mu}\left(\gamma_{\mu}\right), \gamma_{\mu}\right) \geq$ $d\left(h_{\mu}\left(\gamma_{\mu}\right), \mathcal{B}(r, \beta) \backslash \mathcal{V}_{\mu}\right)>C_{\mathcal{V}}$

Sketch of Proof. Firstly, in order to prove that $h_{\mu}\left(\mathcal{V}_{\mu}\right) \subset \mathcal{V}_{\mu}$ consider a point $\xi \in \partial \mathcal{V}_{\mu}=\gamma_{\mu}$. The vector $h_{\mu}(\xi)-\xi$ is the tangent vector at the point $\xi$ which lies on the logarithmic spiral starting at the fixed point $\xi_{0}$. Then the vector $h_{\mu}(\xi)-\xi$ points into the interior of $\mathcal{V}_{\mu}$, proving the first claim.

Secondly, let $\mu_{3} \in\left(0, \mu_{2}\right)$ and $\delta_{3}=\delta_{3}\left(\mu_{3}\right)$ as described in the lemma. We claim that $\gamma_{\mu} \cap D(0, r)=\emptyset$. This follows from an approximation of $R_{\mu}$. On the one hand, recall that $\zeta_{2}=a^{-1} \cdot L \cdot(-1+i \tan \eta)$, with $a \in(0,1)$. Then there exists some positive constant $L^{\prime}$ such that $\left|\zeta_{2}\right|=a^{-1} \cdot L^{\prime}$. On the other hand, using the inequation $0 \leq \arg \left(\left(1-M_{\mu}\right)^{-1}\right) \leq \beta$ together with $0 \leq \arg \left(M_{\mu}\right) \leq \frac{\eta}{2}$ implies $0 \leq \arg \left(\xi_{0}\right) \leq \pi-\eta$. Hence it is possible to find a constant $C_{\mathcal{V}}>1$ and $0<\delta_{3}<\frac{1}{2}$ such that $R_{\mu} \geq a^{-1} \cdot C_{\mathcal{V}}$. For such a $\delta_{3}$ we can find a $r>0$ such that the claim holds.

Finally, the distance $d\left(h_{\mu}\left(\gamma_{\mu}\right), \gamma_{\mu}\right)$ can be computed by evaluating the expression $\left|h_{\mu}(\xi)-\xi_{0}\right|-R_{\mu}$ for some $\xi=\xi_{0}+R_{\mu} e^{i t} \in \gamma_{\mu}$. Since $\left|h_{\mu}(\xi)-\xi_{0}\right|=\frac{R_{\mu}}{\left|M_{\mu}\right|}$, we have $\left|h_{\mu}(\xi)-\xi_{0}\right|-R_{\mu}=\frac{R_{\mu}\left(1-M_{\mu}\right)}{\left|M_{\mu}\right|} \geq \frac{\left(1-\delta_{3}\right) \cdot C_{\mathcal{V}}}{\delta_{3}\left|M_{\mu}\right|}$.

To conclude the proof of the invariance of any compact set $\phi(K)$ under $f_{\mu}$ we define the sets

$$
\mathcal{W}_{\mu}:=\mathcal{B}(r, \beta) \cup \mathcal{V}_{\mu} \quad \text { and } \quad \mathcal{W}_{\mu}^{\prime}:=\left\{\xi: \rho(\xi) \in \mathcal{W}_{\mu}\right\}
$$

for every $\mu \leq \mu_{3}$. The lemma below follows directly from Lemmas 3.23 and 3.24 . Lemma 3.25. For every $M_{\mu}$ such that $\left|M_{\mu}-M_{0}\right|<\delta$ with $\delta=\delta\left(\mu_{3}\right)$ it holds that $h_{\mu}\left(\mathcal{W}_{\mu}\right) \subset \mathcal{W}_{\mu}$ and $d\left(h_{\mu}\left(\mathcal{W}_{\mu}\right), \partial \mathcal{W}_{\mu}\right)>\min \left\{C_{\mathcal{B}}, C_{\mathcal{V}}\right\}$

The function $f_{\mu}$ differs from the function $h_{\mu}$ on the terms of order $|\xi|^{-\frac{1}{m}}$ only. Thus there exists some $r>0$ sufficiently large such that for every $\xi \in \Omega_{r}$ we obtain $\left|\rho\left(f_{\mu}(\xi)\right)-h_{\mu}(\rho(\xi))\right|<\min \left\{C_{\mathcal{B}}, C_{\mathcal{V}}\right\}$. Using Lemma 3.25 it immediately follows that $\mathcal{W}_{\mu}^{\prime}$ is invariant under $f_{\mu}$ and we are now ready to prove the Main Lemma.

Proof of Main Lemma 3.20. Let $z_{p}$ and $z_{p, \mu}$ be fixed points of $g$ and $g_{\mu}$ respectively. Lemma 3.10 implies that $z_{p, \mu} \rightarrow z_{p}$ as $\mu$ tends to zero. Then for every 
small neighbourhood $U$ of $z_{p}$ there exists an $\mu_{1} \in\left(0, \frac{1}{2}\right)$ such that $z_{p, \mu} \in U \cap \mathcal{D}_{p}^{*}$ for every $\mu \leq \mu_{1}$.

Consider an arbitrary but fixed small neighbourhood $U$ of $z_{p}$. Now consider a corresponding neighbourhood of infinity $\Omega_{r}$ with $r$ sufficiently large. We showed above that given any compact $K \subset U \cap \mathcal{D}_{p}^{*}$, then $g_{0}^{n}(K) \subset \subset U \cap \mathcal{D}_{p}^{*}$, which in turn implies $f_{0}^{n}(K) \subset \phi(K)$. We also proved for $\phi(K) \subset \mathcal{B}^{\prime}(r, \beta) \subset \Omega_{r}$ that $\mathcal{B}^{\prime}(r, \beta)$ is invariant under $f_{0}$.

In order to prove the invariance under $f_{\mu}$ we constructed a larger domain $\mathcal{W}_{\mu}:=$ $\mathcal{B}(r, \beta) \cup \mathcal{V}_{\mu}$, with $\mathcal{B}(r, \beta)=\rho\left(\mathcal{B}^{\prime}(r, \beta)\right)$. We have shown that $\mathcal{W}_{\mu}$ is invariant under the function $h_{\mu}=M_{\mu}^{-1} \xi-1$ for every $\mu \leq \mu_{*}$.

Since the function $h_{\mu}$ satisfies $d\left(h_{\mu}\left(\mathcal{W}_{\mu}\right), \partial \mathcal{W}_{\mu}\right)>C_{*}$, for some positive constant $C_{*}$ and $f_{\mu}$ is a small perturbation of $h_{\mu}$, the invariance of $\mathcal{W}_{\mu}^{\prime}$ is preserved by $f_{\mu}$. Then the domain $\mathcal{U}_{\mu}:=\phi^{-1}\left(\mathcal{W}_{\mu}^{\prime}\right)$ is invariant under $g_{\mu}$ and with this the compact set $K$ is a subset of $U \cap \mathcal{A}_{p, \mu}^{*}$ for every $\mu \leq \mu_{3}$.

To prove the kernel convergence recall the analytic representation $g_{\mu}(z)=M_{\mu} z+$ $a_{m+1} z^{m+1}$, with $M_{0}=1$ for $\mu=0$ and $M_{\mu} \in \mathbb{D}$ for $\mu>0$. Notice that this reparametrisation indicates the number of petals of the parabolic basin of $g_{0}$, namely $m$ petals.

Proof of Theorem 3.18. Let $\mu_{1} \in\left(0, \frac{1}{2}\right)$ be a parameter such that the convergence $z_{p, \mu} \rightarrow z_{p}$ holds and $c_{0}$ be the free critical point contained in $\mathcal{D}_{p}^{*}$. It is well-known that $g_{0}$ maps the petals on each other building a cycle. Denote the petals by $P_{j}$, $j=0, \ldots, m-1$, such that $c_{0} \in P_{0}$ and $c_{j}:=g_{0}^{j}\left(c_{0}\right) \in P_{j}$. We claim that $\bigcup_{j=0}^{m-1} P_{j}$ is a kernel of the basins of attraction $\mathcal{A}_{p, \mu}^{*}$. This means that each petal $P_{j}$ is a kernel of the basins distinguished by the marked point $c_{j}$.

We firstly prove that the points $c_{j}$ belong to $\mathcal{A}_{p, \mu}$ for all $\mu \leq \mu_{1}$. The set of critical points for $g_{0}$ and $g_{\mu}$ are equal. It follows that $c_{0}$ is a critical point of $g_{\mu}$ too and, in particular, it is contained in $\mathcal{A}_{p, \mu}^{*}$. We claim that $c_{j} \in \mathcal{A}_{p, \mu}^{*}$ for every $\mu \leq \mu_{1}$ as well. Using the uniform convergence we can find a $\delta=\delta(\mu, n)>0$ for every fixed $\mu$ such that $\left|g_{\mu}\left(c_{0}\right)-g_{0}\left(c_{0}\right)\right|<\delta$ together with $\left|g_{\mu}^{n}\left(c_{0}\right)-g_{0}^{n}\left(c_{0}\right)\right|<\delta$ for $n=2, \ldots, m-1$. Hence $g_{0}^{n}\left(c_{0}\right) \in \mathcal{A}_{p, \mu}^{*}$ for every $\mu<\mu_{1}$ and $n=1, \ldots, m-1$. It follows by construction that each marked point $c_{j}$ belongs to a petal $P_{j}$, proving the condition on marked points.

We proved in the Main Lemma 3.20 that any given compact subset $K \subset U \cap \mathcal{D}_{p}^{*}$ is also contained in $\mathcal{A}_{p, \mu}^{*}$ for every $\mu \leq \mu_{*}$. It implies the condition on compact sets for the kernel convergence for compact sets $K_{j}:=K \cap P_{j}$ for every $j \in\{0, \ldots, m-1\}$. 
Finally we prove that $\bigcup_{j=0}^{m-1} P_{j}$ is a kernel of the basins of attraction for any subsequence of parameters in $\left(0, \mu_{1}\right)$. For this, it is enough to show that if there exists an open set $U$ and a $\mu_{2}$ such that $U \subset \mathcal{A}_{p, \mu}^{*}$ for every $\mu \leq \mu_{2}$ then $U \subset$ $\bigcup_{j=0}^{m-1} P_{j}$ is satisfied as well.

Assume $U \nsubseteq \bigcup_{j=0}^{m-1} P_{j}$. Then there exists $z_{0} \in U$ such that $z_{0} \notin P_{j}$ for some $j$. On the one hand $z_{0} \in U \subset \mathcal{A}_{p, \mu}^{*}$. For each fixed $\mu$ we have that $g_{\mu}^{n}\left(z_{0}\right)$ converges to $z_{p, \mu}$ as $n \rightarrow \infty$. Since $U \subset \mathcal{F}\left(g_{\mu}\right)$ and the iterates $g_{\mu}^{n}$ form a normal family then there exist an $\varepsilon=\varepsilon(n, \mu)$ and $\delta=\delta(\varepsilon)>0$ such that if $U_{\delta}\left(z_{0}\right) \subset U$, then $g_{\mu}^{n}\left(U_{\delta}\left(z_{0}\right)\right) \subset U_{\varepsilon / 2}\left(z_{p, \mu}\right)$, a small neighbourhood of the attracting fixed point $z_{p, \mu}$. In particular this holds for every $\mu \leq \mu_{2}$.

On the other hand $z_{p, \mu}$ convergences to $z_{p}$ as $\mu$ tends to zero. Then there exists $\mu_{3}$ such that $z_{p} \in U_{\varepsilon / 2}\left(z_{p, \mu}\right)$ for all $\mu \leq \mu_{3}$. We obtain that the distance $d\left(g_{\mu}^{n}\left(z_{0}\right), z_{p}\right)<\varepsilon$ and can be arbitrarily small as $n \rightarrow \infty$. This implies that $z_{0}$ is arbitrarily close to $z_{p}$ under iterates. In other words $z_{0}$ is arbitrarily close to an attracting direction of $z_{p}$, which is a contradiction to $z_{0} \notin P_{j}$. We conclude that $U \subset \bigcup_{j=0}^{m-1} P_{j}$ for all $\mu \leq \mu_{*}$ with $\mu_{*}:=\min \left\{\mu_{1}, \mu_{2}, \mu_{3}\right\}$.

We remark that the procedure described above cannot be generalised to parabolic periodic point $z_{p}$ without directly proving it for a given period $n$. Let $\left\{z_{p}\right\}$ denote a parabolic periodic cycle corresponding to a parameter $\lambda_{p}$ on the boundary of an hyperbolic component $\mathcal{H}_{n}$. Such a domain $\mathcal{H}_{n}$ is a component in the copy of the Mandelbrot set for which the free critical point $c_{0}$ belongs to the basin of an attracting periodic cycle of period $n$. Starting with a parameter $\lambda_{p}$, the periodic cycle satisfies $\left|\left(g_{\lambda}^{n}\right)^{\prime}\left(z_{p}\right)\right|=1$. The first difficulty is that we cannot guarantee that a sequence of attracting periodic cycles $\left\{z_{p, \mu}\right\}$ of $g_{\lambda, \mu}$ of the same period $n$ converges to the cycle $\left\{z_{p}\right\}$. However, it still can be possible to find a sequence of attracting cycles for which the convergence holds, but of a different period.

A second difficulty is that the previous arguments may not be computable in an analytic way. That is, the computations for each periodic cycle need not to be explicitly executable, since we search for solutions of the equation $g_{\lambda}^{n}(z)=z$. In the best case there exists an analytic expression for the periodic cycle $\left\{z_{p}\right\}$. Then the approximation through an attracting periodic cycle $\left\{z_{p, \mu}\right\}$ may yield as well very complicated computations.

We claim, for example, that we can approximate the parabolic cycle $\left\{z_{p}\right\}$ by attracting cycles of $(1-\mu) g_{\lambda}^{n}$ instead of taking the $n$-th iterate of $(1-\mu) g_{\lambda}$. But this function is different from $g_{\lambda, \mu}^{n}$ and therefore this is a different story, which has to be told in a different occasion. 


\subsection{Filled Julia sets}

In the case that the Fatou set of the limit function $g_{\lambda}$ has no parabolic cycles and no wandering domains, we are able to prove a slightly stronger result than the Hausdorff convergence of the Julia set. In this section we prove the Hausdorff convergence of the filled Julia set under the assumptions above. Since in this case Siegel discs or other attracting basins are admissible, we use parts of the results stated below to prove Theorem 3.27 on the Hausdorff convergence of Julia sets in the following section.

In Section 3.3 we proved the kernel convergence under the assumption that the Fatou set $\mathcal{F}\left(g_{\lambda}\right)$ consists only of a Baker domain or contains a parabolic basin. We approximated those components by basins of attraction of $g_{\lambda, \mu}$. The cases not considered until now are that $g_{\lambda}$ has an attracting basin $\mathcal{A}_{0}$ containing the unique free critical point $c_{0}$ or a Siegel disc, in which case an irrational fixed point belongs to the Fatou set.

Kriete consider in [47] a family of meromorphic functions $f_{n}$ converging uniformly on $\mathbb{C}$ to a meromorphic function $f$. He proved the Hausdorff convergence of the filled Julia set, when $f$ fulfils certain conditions, that is, when $f$ has no wandering domains, Baker domains or rationally indifferent cycles. In our case, the functions $g_{\lambda}$ do not fulfil these conditions. Hence we can not directly apply his result but we are able to adapt his definitions and results for our particular case. We begin by defining the filled Julia set in an analogue way to the well-known filled Julia set.

In the case of polynomials, $\infty$ is always an attracting fixed point. Therefore the filled Julia set is defined as the complement of the basin of attraction of $\infty$. For rational maps the point at infinity is a regular point and it can present any behaviour. For transcendental entire functions the point at $\infty$ is an essential singularity and is always contained in the Julia set. In our case, $\mathcal{F}\left(g_{\lambda}\right)$ has a Baker domain $\mathcal{B}_{\lambda}$ for every $\lambda$ where the iterates converge to $\infty$. Hence it make sense to define the filled Julia set as the complement of $\mathcal{B}_{\lambda}$

$$
\mathcal{K}\left(g_{\lambda}\right):=\mathbb{C} \backslash \mathcal{B}_{\lambda} .
$$

For the approximating functions $g_{\lambda, \mu}$ we define

$$
\mathcal{K}\left(g_{\lambda, \mu}\right):=\mathbb{C} \backslash \mathcal{A}_{\mu},
$$

where $\mathcal{A}_{\mu}$ is the attracting basin converging to $\mathcal{B}_{\lambda}$ as $\mu$ tends to zero. Note that $\mathcal{K}\left(g_{\lambda}\right)$ and $\mathcal{K}\left(g_{\lambda, \mu}\right)$ contain the Julia sets $\mathcal{J}\left(g_{\lambda}\right)$ and $\mathcal{J}\left(g_{\lambda, \mu}\right)$ respectively. We state the result for these sets as follows. 
Theorem 3.26. Let $\lambda \in \mathbb{C}^{*}$ be arbitrary but fixed such that $g_{\lambda}$ has no wandering domains and no parabolic cycles. Then $\mathcal{K}\left(g_{\lambda, \mu}\right)$ converges to $\mathcal{K}\left(g_{\lambda}\right)$ in the Hausdorff metric as $\mu$ tends to zero.

Proof. Recall that $\mathcal{K}\left(g_{\lambda}\right)$ and $\mathcal{K}\left(g_{\lambda, \mu}\right)$ are closed and completely invariant under $g_{\lambda}$ and $g_{\lambda, \mu}$ respectively. From the Kernel convergence of $\mathcal{A}_{\mu}$ to $\mathcal{B}_{\lambda}$ there exists a $\mu_{*}$ such that if an open set $U \subset \mathcal{B}_{\lambda}$, then $U$ is contained in $\mathcal{A}_{\mu}$ for every $\mu \leq \mu_{*}$. For some $\varepsilon>0$ consider the neighbourhood $U_{\varepsilon}\left(\mathcal{K}\left(g_{\lambda}\right)\right)$. The latter argument implies that $\mathcal{K}\left(g_{\lambda, \mu}\right) \subset U_{\varepsilon}\left(\mathcal{K}\left(g_{\lambda}\right)\right)$ for every $\mu \leq \mu_{*}$. Therefore to prove the Hausdorff convergence we only need to show that $\mathcal{K}\left(g_{\lambda}\right) \subset U_{\varepsilon}\left(\mathcal{K}\left(g_{\lambda, \mu}\right)\right)$ for any given $\varepsilon>0$.

Assume that this is false. Then there exists some $\varepsilon>0$ and $\mu_{0} \in\left(0, \frac{1}{2}\right)$ such that $\mathcal{K}\left(g_{\lambda}\right) \nsubseteq U_{\varepsilon}\left(\mathcal{K}\left(g_{\lambda, \mu}\right)\right)$ for every $\mu \leq \mu_{0}$. Since $\mathcal{K}\left(g_{\lambda}\right)$ has no isolated points (otherwise $\mathcal{J}\left(g_{\lambda}\right)$ would have some) there exists $z_{0} \in \mathcal{K}\left(g_{\lambda}\right)$ with $U_{\varepsilon / 2}\left(z_{0}\right) \cap \mathcal{K}\left(g_{\lambda}\right) \neq$ $\emptyset$ and $U_{\varepsilon / 2}\left(z_{0}\right) \cap \mathcal{K}\left(g_{\lambda, \mu}\right)=\emptyset$ for every $\mu \leq \mu_{0}$. Let $\left\{\mu_{k}\right\}_{k \in \mathbb{N}}$ be an arbitrary sequence of parameters in $\left(0, \mu_{0}\right]$ such that $\mu_{k} \rightarrow 0$ as $k \rightarrow \infty$. For each $\mu_{k}$ we consider points $z_{\mu_{k}} \in \mathcal{K}\left(g_{\lambda, \mu_{k}}\right)$ and define the set of accumulation points of sequences $\left\{z_{\mu_{k}}\right\}_{k \in \mathbb{N}}$ denoted by $\mathcal{E}$.

We have that $\mathcal{E} \subset \mathcal{K}\left(g_{\lambda}\right)$ but $\mathcal{E} \neq \mathcal{K}\left(g_{\lambda}\right)$. Using the lower-semicontinuity described in Section 1.9 we obtain that $\mathcal{J}\left(g_{\lambda}\right) \subset U_{\varepsilon}\left(\mathcal{J}\left(g_{\lambda, \mu}\right)\right)$ for each given $\varepsilon>0$ and every $\mu \leq \mu_{0}$. Combining the three previous statements yields $\mathcal{J}\left(g_{\lambda}\right) \subset \mathcal{E}$, recalling that $\mathcal{J}\left(g_{\lambda}\right)=\partial \mathcal{K}\left(g_{\lambda}\right)$.

Thus, there exists some domain $G \subset \subset \mathcal{K}\left(g_{\lambda}\right) \backslash \mathcal{E}$ such that $G \cap \mathcal{K}\left(g_{\lambda, \mu}\right)=\emptyset$ for all $\mu \leq \mu_{0}$. By assumption $g_{\lambda}$ has no wandering domains, parabolic cycles and any further Baker domain different from $\mathcal{B}_{\lambda}$. Since $G \cap \mathcal{J}\left(g_{\lambda}\right)=\emptyset$ and $\mathcal{K}\left(g_{\lambda}\right)$ and $\mathcal{E}$ are invariant under $g_{\lambda}$ we may assume that $G$ lies in a periodic component of $\mathcal{F}\left(g_{\lambda}\right)$. After switching to an appropriate iterate of $g_{\lambda}$ we may assume that $G$ lies in an invariant component of $\mathcal{F}\left(g_{\lambda}\right)$. The following two cases may occur.

i) $G \subset \subset \mathcal{A}$ for some component of $\mathcal{F}\left(g_{\lambda}\right)$ containing an attracting periodic point $z_{0}$, or

ii) $G$ lies in a Siegel disc of $\mathcal{F}\left(g_{\lambda}\right)$.

Now we consider both cases separately.

i) We have that $G \subset \subset \mathcal{A} \subset \operatorname{int}\left(\mathcal{K}\left(g_{\lambda}\right)\right)$ and $G \subset \subset \widehat{\mathbb{C}} \backslash \mathcal{J}\left(g_{\lambda}\right) \subset \mathbb{C}$. Since $G$ is not contained in $\mathcal{B}_{\lambda}$, then $G \subset \mathcal{A}_{0}$, where $\mathcal{A}_{0}$ is the basin of attraction containing the unique free critical point $c_{0}$. Using the persistence of attracting cycles, we conclude that $G$ lies in the basin of some attracting periodic point $z_{0, \mu}$ of 
$g_{\lambda, \mu}$ different from $\mathcal{A}_{\mu}$. In other words, $G \subset \mathcal{A}_{0, \mu}$, the basin of attraction of $z_{0, \mu}$, and $G \subset \widehat{\mathbb{C}} \backslash \mathcal{A}_{\mu}=\mathcal{K}\left(g_{\lambda, \mu}\right)$ for every $\mu \leq \mu_{0}$, which is a contradiction.

ii) If $G$ lies in a Siegel disc $\mathcal{S}$, then there exists some $m \in \mathbb{N}$ such that after $m$ iterates $\bigcup_{k=0}^{m} g_{\lambda}^{m}(G) \subset \subset \mathcal{K}\left(g_{\lambda}\right) \backslash \mathcal{K}\left(g_{\lambda, \mu}\right)$ contains a curve $\gamma$ invariant with respect to $g_{\lambda}$. On the one hand, this implies that $\gamma$ and $g_{\lambda}(\gamma)$ are homotopic in $\mathbb{C} \backslash \mathcal{K}\left(g_{\lambda, \mu}\right)$ for every $\mu \leq \mu_{0}$. This in turn implies that $\gamma$ and $g_{\lambda, \mu}(\gamma)$ are also homotopic in $\mathbb{C} \backslash \mathcal{K}\left(g_{\lambda, \mu}\right)$ for every $\mu \leq \mu_{0}$. Then $g_{\lambda, \mu}(\gamma)$ and $g_{\lambda, \mu}^{2}(\gamma)$ are homotopic in $\mathbb{C} \backslash g_{\lambda, \mu}\left(\mathcal{K}\left(g_{\lambda, \mu}\right)\right)=\mathbb{C} \backslash \mathcal{K}\left(g_{\lambda, \mu}\right)$. Continuing inductively, we obtain that $\gamma$ is homotopic to $g_{\lambda, \mu}^{k}(\gamma)$ in $\mathbb{C} \backslash \mathcal{K}\left(g_{\lambda, \mu}\right)$ for every $k \in \mathbb{N}$. On the other hand, $\gamma \subset \mathcal{A}_{\mu}=\mathbb{C} \backslash \mathcal{K}\left(g_{\lambda, \mu}\right)$. Then $g_{\lambda, \mu}^{k}(\gamma)$ is homotopic to a point for sufficiently large $k$. Finally $\gamma$ is homotopic to a point in $\mathbb{C} \backslash \mathcal{K}\left(g_{\lambda, \mu}\right)$.

Denote by $G_{1}$ the component of $\widehat{\mathbb{C}} \backslash \gamma$ containing $\partial S$ and by $G_{2}$ the other component. In particular, $G_{2}$ contains the centre $\xi_{2}$ of the Siegel disc $\mathcal{S}$, which is an irrational fixed point of $g_{\lambda}$. Recall that $\partial \mathcal{S} \subset \mathcal{J}\left(g_{\lambda}\right)$. The density of repelling periodic points in the Julia set implies the existence of a repelling point $\zeta_{1} \in G_{1} \cap \mathcal{J}\left(g_{\lambda}\right)$. Now, Rouché's Theorem yields the existence of repelling periodic points $\zeta_{\mu}$ of $g_{\lambda, \mu}$ in $G_{1}$ for every $\mu \leq \mu_{0}$ such that $\lim _{\mu \rightarrow 0} \zeta_{\mu}=\zeta_{1}$.

We have that $\gamma \subset \mathbb{C} \backslash \mathcal{K}\left(g_{\lambda, \mu}\right)=\mathcal{A}_{\mu}$ which implies $G_{2} \subset \mathcal{A}_{\mu}$. The homotopy of $\gamma$ to a point in $\mathbb{C} \backslash \mathcal{K}\left(g_{\lambda, \mu}\right)$ together again with Rouché's Theorem implies the existence of fixed points $\xi_{\mu}$ of $g_{\lambda, \mu}$ which converge to $\xi_{2}$. The latter implies $\xi_{\mu} \in G_{2}$ for all $\mu \leq \mu_{0}$. Hence $\xi_{\mu} \in \mathcal{A}_{\mu}$ and each fixed point $\xi_{\mu}$ must coincide with the attracting fixed point $z_{\mu}$. We obtain $\lim _{\mu \rightarrow 0} z_{\mu}=\lim _{\mu \rightarrow 0} \xi_{\mu}=\xi_{2}$, a contradiction.

The theorem above shows that the biggest difficulty to prove convergence in a dynamical sense comes from the presence of wandering domains or parabolic cycles in the Fatou set of the limit function. In the present case the convergence of the filled Julia set $\mathcal{K}\left(g_{\lambda, \mu}\right)$ to $\mathcal{K}\left(g_{\lambda}\right)$ implies automatically the convergence of the (usual) Julia sets under the assumption that $g_{\lambda}$ has no parabolic cycles and the Siegel disc is of period one.

Remark 3.2. Concerning the case that $g_{\lambda}$ has a Siegel disc $\mathcal{S}$, note that the proof above does not specify through which kind of Fatou component it is approximated. If the centre of $\mathcal{S}$ is an indifferent fixed point, we can approximate it through basins of attracting fixed points. Nevertheless, if the centre is an indifferent period point, we cannot guarantee the existence of a sequence of attracting periodic points 
converging to the centre having the same period, analogously to the case of parabolic periodic points.

In the light of Kriete's result in [47] we could extend his result since we control the existing Baker domain. In other words, the kernel convergence of $\mathcal{A}_{\mu}$ to $\mathcal{B}_{\lambda}$ guarantees the convergence of their complements in the plane, the filled Julia sets, under the restrictions mentioned above. The second reason that allows to extend the result is that the Fatou set $\mathcal{F}\left(g_{\lambda}\right)$ contains no other Baker domain different from $\mathcal{B}_{\lambda}$. Therefore it is important to be able to control and to have convergence on any existing Baker domain.

\subsection{Hausdorff convergence of Julia sets}

The main result of the present work is the Hausdorff convergence of $\mathcal{J}\left(g_{\lambda, \mu}\right)$ to $\mathcal{J}\left(g_{\lambda}\right)$, which is achieved under certain conditions. A first example where such convergence was successful is due to Devaney et. al presented in [20]. They showed convergence on subsets of the parameter plane of polynomials $P_{\lambda, d}(z)=\lambda\left(1+\frac{z}{d}\right)^{d}$ and the exponential family $E_{\lambda}(z)=\lambda e^{z}$. Krauskopf proved in [41] that $\mathcal{J}\left(P_{\lambda, d}\right)$ converges to $\mathcal{J}\left(E_{\lambda}\right)$ if the Fatou set of $E_{\lambda}$ consists only of basins of attraction or if it is empty.

Such convergences are not guarantee in general for families of functions $f_{n}$ converging uniformly on compact subset to $f$. Let $f$ and $\left\{f_{n}\right\}_{n \in \mathbb{N}}$ be meromorphic functions such that $f_{n} \rightarrow f$ as $n \rightarrow \infty$. The Hausdorff convergence of $\mathcal{J}\left(f_{n}\right)$ to $\mathcal{J}(f)$ can be assured if the Fatou set of the limit function $f$ consists only of basins of attraction and $\infty \in \mathcal{J}(f)$, as it was proved by Krauskopf and Kriete in [44]. This result was previously proved for entire functions by Kisaka in [36]. In contrast to this, if $f$ has a Baker domain or other Fatou components there exist several counterexamples, as those showed by Lauber in [48, 49] or by Krauskopf and Kriete in [42, 43], among others.

We present in this section some minimal conditions such that the Hausdorff convergence of the Julia set $\mathcal{J}\left(g_{\lambda, \mu}\right)$ to $\mathcal{J}\left(g_{\lambda}\right)$ applies. One of the main tools in the proof is the kernel converge of absorbing domains when $g_{\lambda}$ has a Baker domain or a parabolic basin, presented in Section 3.3. The rest of the arguments follow from the convergence of the filled Julia sets, presented in Section 3.4 .

In order to state the main theorem we define an analogue concept to hyperbolic components in the parameter plane of the functions $g_{\lambda}$. Consider a family of entire functions of finite type, that is, functions $f$ such that $\operatorname{sing}\left(f^{-1}\right)$ consists 
of finitely many points. An entire function $f$ of finite type is called hyperbolic if $P(f)=O^{+}\left(\operatorname{sing}\left(f^{-1}\right)\right)$ is relatively compact in $\mathcal{F}(f)$. If a family of functions $F_{\beta}$ depends analytically on a parameter $\beta \in \mathbb{C}$, then an hyperbolic component $H$ is defined as the set of parameters $\beta$ for which the functions $f_{\beta}$ are hyperbolic. It is well-known that $H$ is an open subset of the parameter plane and may consist of infinitely many components. This definition applies as well for meromorphic functions.

The family of functions $\mathcal{G}_{\lambda}$ is not of finite type and is never hyperbolic, since $P(f) \rightarrow \infty \in \mathcal{J}\left(g_{\lambda}\right)$ for every singular value, except at most one. Therefore $P(f)$ cannot be relatively compact in the Fatou set. Even though the Fatou set of the family $\mathcal{G}_{\lambda, \mu}$ may consist only of basins of attraction, we cannot guarantee that $P(f)$ satisfies the conditions for hyperbolicity, since we do not control all singular values. However we can distinguish parameters $\lambda$ for $\mathcal{G}_{\lambda}$ in the following manner. Let $c_{0}$ be the free critical point of $g_{\lambda}, \mathcal{B}_{\lambda}$ the Baker domain and $\mathcal{A}_{n}$ a basin of attraction of period $n$. We define the following subsets of the parameter plane

$$
\begin{aligned}
& \mathcal{H}_{\mathcal{B}}:=\left\{\lambda \in \mathbb{C}^{*} \mid c_{0} \in \mathcal{B}_{\lambda}\right\} \quad \text { and } \\
& \mathcal{H}_{n}:=\left\{\lambda \in \mathbb{C}^{*} \mid c_{0} \in \mathcal{A}_{n}\right\} .
\end{aligned}
$$

Lauber proved in [48] that the set $\mathcal{H}_{\mathcal{B}}$ is open and all components of $\mathcal{H}_{\mathcal{B}} \cup\{0\}$ are simply connected. Furthermore, he proved as well that the parameter plane of $g_{\lambda}$ contains a copy of the Mandelbrot set $\mathcal{M}$. Hence the components $\mathcal{H}_{n}$ correspond to the hyperbolic components of $\mathcal{M}$. It is well-known that each domain $\mathcal{H}_{n}$ is open.

If $\lambda \in \overline{\mathcal{H}_{n}}$, then $g_{\lambda}$ has a non-repelling periodic point $z$, that is, $\left|\left(g_{\lambda}^{n}\right)^{\prime}(z)\right| \leq 1$. In the case that $z_{p}$ is an indifferent fixed point, it means that $\lambda \in \partial \mathcal{H}_{1}$, we explicitly compute in Section 3.2 an expression for the fixed point $z_{p}$. Furthermore, we give a parametrisation of the curve $\partial \mathcal{H}_{1}$ for these parameters $\lambda$ depending on $z_{p}$. In this case, the parametrisation of $\partial \mathcal{H}_{1}$ has a singularity at $z_{p}=-1$ and the corresponding parameter $\lambda_{p}=-e$.

For higher period $n$ the boundary of $\mathcal{H}_{n}$ has at most countably many singularities which we call corners and use the notation

$$
\mathcal{C}:=\left\{\text { singularities of } \partial \mathcal{H}_{n}\right\} .
$$

In particular $\mathcal{C}$ is a discrete subset of $\partial \mathcal{H}_{n}$. Recall that for parameters $\lambda \in \partial \mathcal{H}_{n}$ one of the following possibilities occurs

- $g_{\lambda}$ has a cycle of rational indifferent points lying in $\mathcal{J}\left(g_{\lambda}\right)$ coming with a parabolic basin $\mathcal{D}_{p}$. 
- $g_{\lambda}$ has a Siegel disc $\mathcal{S}$, with a linearisable irrational periodic point in $\mathcal{F}\left(g_{\lambda}\right)$ as the centre of $\mathcal{S}$.

- $g_{\lambda}$ has a Cremer point, that is, a non-linearisable irrational periodic point contained in $\mathcal{J}\left(g_{\lambda}\right)$.

In the latter case, the Fatou set has no other non-repelling cycle than the Baker domain, as we showed in Section 3.1. Now we state the conditions for the convergence of the Julia sets.

Theorem 3.27 (Hausdorff Convergence Theorem). Let $\lambda \in \mathbb{C}^{*}$ and $\mathcal{H}:=\bigcup_{n \in \mathbb{N}} \mathcal{H}_{n}$. If the function $g_{\lambda}$ satisfies one the following

i) $\mathcal{F}\left(g_{\lambda}\right)$ consists only of the Baker domain $\mathcal{B}_{\lambda}$,

ii) $g_{\lambda}$ has an attracting periodic cycle (of any period),

iii) $g_{\lambda}$ has a Cremer cycle (of any period),

iv) $g_{\lambda}$ has a Siegel disc, which centre is a fixed point, or

v) $g_{\lambda}$ has a parabolic fixed point for $\lambda \in \partial \mathcal{H}_{1}$,

then $\mathcal{J}\left(g_{\lambda, \mu}\right)$ converges to $\mathcal{J}\left(g_{\lambda}\right)$ in the Hausdorff metric as $\mu$ tends to zero.

Proof. At first recall that for parameters $\lambda \in \mathcal{H}_{\mathcal{B}} \cup \overline{\mathcal{H}}$ we proved in Corollary 3.8 that $g_{\lambda}$ cannot have wandering domains. This include all cases listed above. Let $\varepsilon>0$ be arbitrary but fixed. The lower-semicontinuity implies the existence of a $\mu_{0}$ such that $\mathcal{J}\left(g_{\lambda}\right) \subset U_{\varepsilon}\left(\mathcal{J}\left(g_{\lambda, \mu}\right)\right)$ for every $\mu \leq \mu_{0}$.

The inverse inclusion remains to be shown, that is, $J\left(g_{\lambda, \mu}\right) \subset U_{\varepsilon}\left(\mathcal{J}\left(g_{\lambda}\right)\right)$. Write $U_{\varepsilon}:=U_{\varepsilon}\left(\mathcal{J}\left(g_{\lambda}\right)\right)$ for the $\varepsilon$-neighbourhood of the Julia set, and $K_{\varepsilon}:=\widehat{\mathbb{C}} \backslash U_{\varepsilon}$ for its complement. Notice that $U_{\varepsilon}$ may consist of several connected components. Moreover $K_{\varepsilon}$ is a compact subset of $\mathbb{C}$.

We claim that $K_{\varepsilon}$ consists only of finitely many components. If $\mathcal{F}\left(g_{\lambda}\right)=\mathcal{B}_{\lambda}$, then it is completely invariant and $K_{\varepsilon}$ consists only of one component, since all critical points are contained in $\mathcal{B}_{\lambda}$. If $\mathcal{F}\left(g_{\lambda}\right)$ has other components beside the Baker domain $\mathcal{B}_{\lambda}$, assume that our claim is false. Since $K_{\varepsilon}$ is bounded, an infinite number of its components accumulate at some point $\zeta_{0}$. Assume these are covered by one element of a finite subcovering, says by $V$. Then the diameter of the components of $K_{\varepsilon}$ as well as the diameter of the complement of $K_{\varepsilon}$ contained in $V$ tend to zero. This is a contradiction to the fact that $U_{\varepsilon}$ is an $\varepsilon$-neighbourhood for some fixed $\varepsilon$. 
Since $K_{\varepsilon}$ is contained in $\mathcal{F}\left(g_{\lambda}\right), K_{\varepsilon}$ intersects only finitely many connected components of it. We continue by individually considering the cases of the possible different components of $\mathcal{F}\left(g_{\lambda}\right)$.

i) If $\mathcal{F}\left(g_{\lambda}\right)=\mathcal{B}_{\lambda}$ then, as mentioned above, $K_{\varepsilon}$ consists only of one component and it is contained in $\mathcal{B}_{\lambda}$. The result follows immediately from the Kernel convergence of the basins of attraction $\mathcal{A}_{\mu}$ to $\mathcal{B}_{\lambda}$ proved in Theorem 3.14 and Lemma 3.16. Therefore $K_{\varepsilon} \subset \mathcal{A}_{\mu}$ for every $\mu \leq \mu_{0}$.

ii) If $c_{0}$ belongs to a basin of attraction $\mathcal{A}_{0}$, then there does not exist a parabolic basin nor a Siegel disc. Furthermore, the persistence of attracting cycles stated as Theorem 1.9 implies the existence of sequence of attracting periodic points $z_{0, \mu}$ converging to a given cycle of any period. Then we use the convergence of the filled Julia set proved in Theorem 3.26 to complete the result. The latter result implies in particular that $K_{\varepsilon}$ is contained in $\mathcal{F}\left(g_{\lambda, \mu}\right)$.

iii) This case reduces to case i), since if $g_{\lambda}$ has a Cremer point, there does not exist any other Fatou component beside $\mathcal{B}_{\lambda}$ (in particular no wandering domain).

iv) In the case the free critical point lies in a Siegel $\operatorname{disc} \mathcal{S}$, again there does not exist a parabolic basin. We use again the convergence of the filled Julia set proved in Theorem 3.26 . But in contrast to the attracting case, we can just assure the convergence if the centre of $\widehat{\mathbb{C}}$ is a fixed point and therefore approximated by attracting fixed points of $g_{\lambda, \mu}$. We refer the reader to Remark 3.2 for a detailed discussion on this.

v) If $\mathcal{F}\left(g_{\lambda}\right)$ contains a parabolic basin $\mathcal{D}_{p}$ in addition to $\mathcal{B}_{\lambda}$, then a finite number of components of $K_{\varepsilon}$ are contained in $\mathcal{D}_{p}$. Analogously, we apply the kernel convergence of the basins of attraction $\mathcal{A}_{p, \mu}$ to $\mathcal{D}_{p}$ proved in Theorem 3.18 . For this we apply Lemma 3.20 a finite number of times. Using the property on compact subset of the kernel convergence for the Baker domain and the parabolic basin we obtain that if $K_{\varepsilon} \subset \mathcal{B}_{\lambda} \cup \mathcal{D}_{p}$ then $K_{\varepsilon} \subset \mathcal{A}_{\mu} \cup \mathcal{A}_{p, \mu}$.

In each of the cases above we obtain $\mathcal{J}\left(g_{\lambda, \mu}\right) \subset \widehat{\mathbb{C}} \backslash K_{\varepsilon}=U_{\varepsilon}\left(\mathcal{J}\left(g_{\lambda}\right)\right)$ for each one of the cases stated in the theorem and every $\mu \leq \mu_{0}$, yielding the desire result.

We remark that the convergence has been proved in the case that $g_{\lambda}$ has a parabolic fixed point and not in a more general case, when $g_{\lambda}$ has a parabolic period point of higher period. Even though this does not imply, the convergence is impossible for parabolic periodic cycles, but each should be proved for each given period $n$ in particular. We proved the dynamical approximation of the parabolic basins by basins of attracting using reparametrisations of the functions $g_{\lambda}$ and $g_{\lambda, \mu}$. The 
reparametrisation may be generalised, but it may be of great difficulty.

Another problem to be considered is that the approximation of the fixed points of the iterate $g_{\lambda}^{n}$ may not be approximated dynamically by the analogue fixed points of an iterate of the same period $g_{\lambda, \mu}^{n}$. In other words, given a parameter $\lambda_{p}$ such that $g_{\lambda_{p}}$ has a parabolic periodic point of period $n$, we cannot guarantee that $g_{\lambda_{p}, \mu}$ has a sequence of periodic points of period $n$ which are attracting and converging to $z_{p}$ as $\mu \rightarrow 0$.

We claim, however, we may achieve the approximation of parabolic periodic points of a given period $n$ by attracting fixed points of the functions $(1-\mu) g_{\lambda}^{n}$, for which the reparametrisations in Section 3.2 may apply. 


\section{Outlook}

We begin by describing some characteristics of the functions we study which cannot be found in the literature. We study the approximation of a transcendental entire function $g_{\lambda}$ that features a Baker domain. We carry out the approximation by a family of transcendental functions too. Most of the examples on convergence and, especially, the first general results were proved for approximations through polynomials. As we mention in the Motivations, the Baker domain $\mathcal{B}_{\lambda}$ is not the result of a logarithmic lift of an attracting basin, which would immediately imply the convergence of $\mathcal{A}_{\mu}$ to $\mathcal{B}_{\lambda}$ by taking the logarithmic change of coordinates of the functions $g_{\lambda}$ and $g_{\lambda, \mu}$. Finally we mention that the existence of further Fatou components enriches the dynamics of both functions, as we can see in the differences between the conditions for having convergence of the filled Julia sets and the (usual) Julia sets.

As we introduce the concept of kernel and Hausdorff convergence in Section 1.9 we mention that these definitions are equivalent considering open sets as complement of compact sets under two conditions: every component in the complement of the compact limit set is a kernel and every kernel of the sequence of open sets is a component of the complement of the compact limit set. In other words, we need to control the Fatou components for all parameters and each of these must be a kernel of the complements of the approximating Julia sets. Since this need not be the case, we prove the Hausdorff convergence of Julia sets directly.

We can interpret the convergence of the filled Julia sets as an approximation from "outside" into the Fatou components. This corresponds to the approach of Gaston Julia to the theory of iteration of functions. An implication is that we can approximate a Siegel disc of any period, since it is not relevant through which kind of Fatou component we approximate it. Even though the approximation for a Siegel disc of period one can follow through basins of attraction, for Siegel discs of higher period this might occur through parabolic basins. 
In other words, we approach the irrational angle of the multiplier through rational angles. Hence we obtain a sequence of parabolic basins for which the number of petals increases and finally tends to infinity as they tend to the Siegel disc. This kind of behaviour is allowed since we consider Fatou components and together with their boundaries. In contrast to this, if we consider the Hausdorff convergence of the (usual) Julia set, such approximation though a sequence of parabolic basins can not occur. Accordingly, we must restrict the Hausdorff convergence of Julia sets only to Siegel discs of period one.

On the contrary, a parabolic basin must be approach from the "inside" in other that the Julia set remains stable. This, in turn, corresponds to the approach of Pierre Fatou. Hence we approximate a rational indifferent fixed point through a sequence of attracting fixed points. And as we mentioned at the end of Section 3.3.2, we have to prove it directly for a given arbitrary period, since we cannot generalise it from the convergence of period one. Therefore we guarantee the Hausdorff convergence of the Julia only if there exists a parabolic basin of period one, and we cannot extended to the filled Julia sets.

Regarding the parameter plane of the functions $g_{\lambda}$, we define in Section 3.5 the domains $\mathcal{H}_{\mathcal{B}}$ and $\mathcal{H}_{n}$ depending on the dynamical property of the free critical point $c_{0}$. We define in an analogous manner in the parameter plane of $g_{\lambda, \mu}$ a domain $\mathcal{H}_{\mathcal{A}}$ of parameters for which $c_{0}$ lies in $\mathcal{A}_{\mu}$, and $\mathcal{H}_{n, \mu}$ if $c_{0}$ lies in a cycle of attracting basins of period n. We claim that $\mathcal{H}_{\mathcal{A}} \cup \mathcal{H}_{n, \mu}$ converges as kernel to $\mathcal{H}_{\mathcal{B}} \cup \mathcal{H}_{n}$ as $\mu$ tends to zero.

An open question is: could we extend the convergence mentioned above to $\overline{\mathcal{H}_{\mathcal{B}}} \cup \overline{\mathcal{H}_{n}}$ as compact subsets of $\widehat{\mathbb{C}}$ ? The release of this question lies in a result due to Krauskopf and Kriete in [43], who proved that the closure of hyperbolic components is approximated by the analogue sets of approximating polynomials in the Hausdorff metric. Their proof heavily depends on the dynamical convergence of the functions for arbitrary but fixed parameters and in the fact that the limit functions are of finite type. In the case of $\overline{\mathcal{H}_{\mathcal{B}}}$ the question is not trivial, since it is well-known that Baker domains are not stable. In our case a problem could arise only if there exist wandering domains for parameters lying in $\partial \mathcal{H}_{\mathcal{B}}$.

At last we refer to the structure of the Julia set $\mathcal{J}\left(g_{\lambda, \mu}\right)$ for points with large imaginary part. It is possible that the critical points which are "trapped" in the chaotic region of $\mathcal{J}\left(g_{\lambda, \mu}\right)$ either lie in the Julia set or in another Fatou component, which must have very small area. An interesting question is if, in this case, the Julia set has positive area, or if these behaviour increases for $\mu \in \mathbb{C}$. However, since this behaviour is produced by the perturbation and the limit functions do not present it, the "chaotic region" vanishes in the limit. 


\section{Acknowledgements}

Firstly, I owe gratitude to my supervisor, Dr. Hartje Kriete, for his great support during this journey. His patience, his time and his teachings are very much appreciated.

I also thank Dr. Victor Pidstrygach for his time and fruitful discussions on my work during the writing phase of the present work. I thank Dr. Halverscheid for his support and encouragement during the final stage of the thesis.

I would like to thank Dr. Nuria Fagella, Dr. Antonio Garijo and Dr. Xavier Jarque for the encouragement on my work during my visit to their research team. I thank the Universitat De Barcelona for financial support during the Spring 2008 through the project "Estudio cualitativo y numérico de sistemas dinámicos en baja dimensión" MTM2005-02139 Ministerio de Educación y Ciencia.

I thank Dr. Pablo Ramacher for the collaboration during the winter semester 2008 through the project "Harmonische Analysis auf affinen G-Varietäten", EmmyNoether-Nachwuchsgruppe RA 1370/2-1. At last, I thank CONACyT for the scholarship in collaboration with DAAD - Referat 414, code number A/02/22770.

On a personal level, I firstly thank my parents, Yolanda and Fernando, for their support and understanding during my youth and adult life. I thank my brother and sisters, Fernando, Fabiola and Dámaris for their love and complicity all these years. Of course I thank my nephews Mateo and Emilio for sharing this life with us, and Margarita, Gerardo and Leonardo. I thank my second family during this period of life, Alex, Ana, Darrin, Gerardo, Yara and Aldo, and specially Thomas and all friends close and far away. I also thank my friends and colleagues Sara, Stefan, Denise, Frau Hartmann and Frau Barann.

And I finally thank all friends I cannot mention here for their time, joy, and for sharing dancing, laughs and coffee and much more during these years. 


\section{Bibliography}

[1] J.M. Aarts and L.G. Oversteegen, The geometry of Julia sets, Trans. Amer. Math. Soc. 338(2) (1993) 897-918.

[2] I.N. Baker, Limit funtions and sets of non-normality in iteration theory, Ann. Acad. Sci. Fenn. Ser. A I 467 (1970) 2-11.

[3] I.N. Baker, The domains of normality of an entire function, Ann. Acad. Sci. Fenn. Ser. A I Math. 1 (1975) 277-283.

[4] I.N. Baker, An entire function which has wandering domains, J. Austral. Math. Soc. Ser. A 22 (1976) 173-176.

[5] I.N. Baker, Infinite limits in the iteration of entire functions, Ergod. Th. and Dynam. Sys., 8 (1988) 503-507.

[6] I.N. Baker, J. Kotus, and Y. Lü, Iterates of meromorphic functions III: Preperiodic domains, Erg. Th. and Dyn. Syst., 11 (1991) 603-618.

[7] I. N. Baker and P. Domínguez, Boundaries of unbounded Fatou components of entire functions, Ann. Acad. Sci. Fenn. Math., 24 (1999) 437-464.

[8] I. N. Baker and J. Weinreich, Boundaries which arise in the dynamics of entire functions, Revue Roumanie Math. Pures Appl., 36 (1991) 413-420.

[9] K. Barański and N. Fagella, Univalent Baker domains, Nonlinearity, 14 (2001) 411-429.

[10] A.F. Beardon. Iteration of rational functions. Springer, (1991).

[11] W. Bergweiler. Invariant domains and singularities. Math. Proc. Camb. Phil. Soc., 117 (1995) 525-532.

[12] W. Bergweiler. Iteration of meromorphic functions. Bull. Amer. Math. Soc., 29(2) (1993) 151-188. 
[13] Walter Bergweiler. On the Julia set of analytic self-maps of the punctured plane. Analysis 15 (1995) 251-256.

[14] W. Bergweiler, M. Haruta, H. Kriete, H.G. Meier, and N. Terglane. On the limit functions of iterates in wandering domains. Annales Academiae Scientiarum Fennicae Series A. I. Math. 18 (1993) 369-375.

[15] L. Carleson and T.W. Gamelin. Complex dynamics. Springer, (1993).

[16] C.C. Cowen, Iteration and the solution of functional equations for functions analytic in the unit disk. Trans. Amer. Math. Soc., 265 (1981) 69-95.

[17] H. Cremer, Über die Schrödersche Functionalgleichung und das Schwarzsche Eckeabbildungsproblem, Sitzungsberg. Math.-phys. Klasse, Ber. Verh. Sächs. Akad. Wiss. Leipzig, 84 (1932) 291-324.

[18] R.L. Devaney, $S e^{x}$ : Dynamics, topology, and bifurcations of complex exponentials, Topology and its Applications 110 (2001) 133-161.

[19] R.L. Devaney and M. Krych, Dynamics of exp(z), Erg. Th. and Dyn. Syst., 4 (1984) 35-52.

[20] R.L. Devaney, L.R. Goldberg, and J.H. Hubbard. A dynamical approximation to the Exponential map by polynomials. Preprint, Berkeley, (1986).

[21] R.L. Devaney and F. Tangerman, Dynamics of entire functions near the essential singularity, Erg. Th. and Dyn. Syst., 6 (1986) 489-503.

[22] A. Douady. Does a Julia set depends continuously on the polynomial?, Am. Math. Soc., Proceedings of Symposia in Applied Mathematics, 49 (1994) 91137.

[23] A. Douady and H. Hubbard. On the dynamics of polynomial-like mappings, Ann. scient. Éc. Norm. Sup., $4^{e}$ série, t. 18 (1985) 287-343.

[24] A. Douady and H. Hubbard. Ètude dynamique des polynômes complexes. Partie II. Publications Mathematiques D'Orsay, 85-4, (1985). PAGES.

[25] A. E. Eremenko and M. Yu. Lyubich. The dynamics of analytic transformations, Leningrad Math. J. Vol. 1, No. 3, 563-634 (1990).

[26] A. E. Eremenko and M. Yu. Lyubich, Dynamical properties of some classes of entire functions, Ann. Inst. Fourier, 41 (1992) 989-1020.

[27] Núria Fagella. Limiting dynamics for the complex standard family, Inter. J. Bifur. Chaos Appl. Sci. Engrg, 5 (3) (1995) 673-699. 
[28] N. Fagella, C. Henriksen, Deformation of entire functions with Baker domains, Discrete Contin. Dis. Syst., 15 (2006) 379-394.

[29] P. Fatou. Sur les équation fonctionelles, Bull. Soc. Math. France 47 (1919) $161-271 ; 48$ (1920) 33-94, 208-314.

[30] P. Fatou, Sur l'itération der fonctions transcendantes entiéres, Acta Math. 47 (1926) 337-360.

[31] G. M. Golusin. Geometrische Funktionentheorie. VEB Deutscher Verlag der Wissenschaften, (1957).

[32] M. Herman, Exemples de fractions rationelles ayant une orbite dense sur la sphère de Riemann, Bull. Soc. Math. France, 11 (1984) 93-142.

[33] A. Hinkkanen, B. Krauskopf, and H. Kriete. Growing a Baker domain from the attracting island, I: The dynamics of the limit function. Mathematica Gottingensis, 17 (1999) 1-44. Revised version (2004).

[34] G. Julia. Sur des problèmes concernant litération des fonctions rationelles. $C$. R. Acad. Sci. Paris Sér. I Math. 166 (1918) 153-156.

[35] G. Julia. Sur litération des fonctions rationelles, J. Math. Pures Appl., 4(7) (1918) 47-245.

[36] Masashi Kisaka. Local uniform convergence and convergence of Julia sets, Nonlinearity, 8 (1995) 273-281.

[37] M. Kisaka, On the connectivity of Julia sets of transcendental entire functions, Ergodic Theory Dinam. Syst., 18 (1998) 189-205.

[38] M. Kisaka and M. Shishikura, On multiply connected wandering domains of entire functions, in "Transcendental Dynamics and Complex Analysis" (Ed. by P. J. Rippon and G. M. Stallard), London Math. Soc. Lec. Note Series 348 (2008) 217-250.

[39] J. Kotus and M. Urbański. The dynamics and geometry of the Fatou functions, Discrete and Continuous Dyn. Sys., 13(2) (2005) 291-338.

[40] H. König. Conformal conjugacies in Baker domains, J. London Math. Soc. (2) 59(1) (1999) 153-170.

[41] Bernd Krauskopf. Convergence of Julia sets in the approximation of $\lambda e^{z}$ by $\lambda[1+(z / d)]^{d}$, Int. J. Bif. Chaos, 3 (1993) 257-270.

[42] B. Krauskopf and H. Kriete. Kernel Convergence of Hyperbolic Components, Ergod. Th. \& Dynam. Sys. 17 (1997) 1137-1146. 
[43] Bernd Krauskopf and Hartje Kriete. Hausdorff convergence and the limit shape of the Unicorns, Experimental Mathematics. 6(2), 117-134, (1997).

[44] B. Krauskopf and H. Kriete. Hausdorff convergence of Julia sets, Bull. Belg. Math. Soc. , 6 (1999) 69-76.

[45] H. Kriete. The stability of Julia sets. Mathematica Gottingensis 22 (1988).

[46] H. Kriete. Über die Stabilität von Julia-Mengen. Dissertation Bremen, (1989).

[47] H. Kriete, Continuity of filled-in Julia sets and the closing lemma. Nonlinearity, 9 (1996) 1599-1608.

[48] A. Lauber. On the stability of functions having Baker domains. Dissertation. Georg-August-Universität, Göttingen, Germany, (2004).

[49] A. Lauber. Bifurcation of Baker domains. Nonlinearity 20 (2007) 1535-1545.

[50] C.T. McMullen, The Mandelbrot set is universal, London Math. Soc. Lecture Note Ser., 274, (2000) 1-17.

[51] J. Milnor. Dynamics of One complex variable. Vieweg, (1999).

[52] M. Misiurewicz, On iterates of $e^{z}$, Erg. Theory and Dyn. Syst., 1 (1981) 103106.

[53] S. Morosawa. The Carathéodory convergence of Fatou components of polynomials to Baker domains or wandering domains, Proc. $2^{\text {nd }}$ ISAAC Cong. Fakuoka, 1 (2000) 347-356

[54] S. Morosawa, Y. Nishimura, M. Taniguchi, T. Ueda, Holomorphic dynamics. Cambridge University Press, (2000).

[55] P.J. Rippon, Baker domains of meromorphic functions, Ergod. Th. and Dynam. Sys., 26 (2006) 1225-1233.

[56] P.J. Rippon, Baker domains, in "Transcendental Dynamics and Complex Analysis" (Ed. by P. J. Rippon and G. M. Stallard), London Math. Soc. Lec. Note Series 348 (2008) 371-395.

[57] P.J. Rippon and G.M. Stallard. Families of Baker domains I, Nonlinearity 12 (1999) 1005-1012.

[58] M. Shishikura. On the quasiconformal surgery of rational functions, Ann. Scient. Éc. Norm. Sup., $4^{e}$ série, t. 20 (1987) 1-29. 